\title{
Determination of the CKM Element V(Ub)
}

\author{
Dominique Fortin
}

SLAC-R-851

Prepared for the Department of Energy

under contract number DE-AC02-76SF00515

Printed in the United States of America. Available from the National Technical Information Service, U.S. Department of Commerce, 5285 Port Royal Road, Springfield, VA 22161. 
This document, and the material and data contained therein, was developed under sponsorship of the United States Government. Neither the United States nor the Department of Energy, nor the Leland Stanford Junior University, nor their employees, nor their respective contractors, subcontractors, or their employees, makes an warranty, express or implied, or assumes any liability of responsibility for accuracy, completeness or usefulness of any information, apparatus, product or process disclosed, or represents that its use will not infringe privately owned rights. Mention of any product, its manufacturer, or suppliers shall not, nor is it intended to, imply approval, disapproval, or fitness of any particular use. A royalty-free, nonexclusive right to use and disseminate same of whatsoever, is expressly reserved to the United States and the University. 


\title{
Determination of the CKM element $\left|V_{u b}\right|$
}

\author{
by \\ Dominique Fortin \\ B.Sc., McGill University, 1998. \\ M.Sc., University of Victoria, 2000. \\ A Dissertation Submitted in Partial Fulfillment of the \\ Requirements for the Degree of \\ DOCTOR OF PHILOSOPHY \\ in the Department of Physics and Astronomy.
}

(C) Dominique Fortin, 2005

University of Victoria

All rights reserved. This dissertation may not be reproduced in whole or in part, by photocopying or other means, without the permission of the author. 
Supervisor: Dr. R.V. Kowalewski

\begin{abstract}
$\underline{\text { Abstract }}$
The precise determination of the CKM matrix element $\left|V_{u b}\right|$ is crucial in testing the Standard Model mechanism for $C P$ violation. From a sample of 88 million $B \bar{B}$ pairs collected with the $B A B A R$ detector, charmless semileptonic $B$ decays are selected using simultaneous requirements on the electron energy, $E_{e}$, and the invariant mass squared of the electronneutrino pair, $q^{2}$. The partial branching fraction, unfolded for detector effects, is determined in a region of the $q^{2}-E_{e}$ plane where the dominating semileptonic decays to charm mesons are highly suppressed. Theoretical calculations based on the Heavy Quark Expansion allows for a determination of $\left|V_{u b}\right|=\left(3.95 \pm 0.27_{-0.42}^{+0.58} \pm 0.25\right) \times 10^{-3}$, where the errors represent experimental, heavy quark parameters and theoretical uncertainties, respectively.
\end{abstract}

Supervisor: Dr. R.V. Kowalewski 


\section{Contents}

Abstract $\quad$ ii

Table of Contents $\quad$ iii

List of Figures $\quad$ vii

List of Tables $\quad$ x

1 Introduction 1

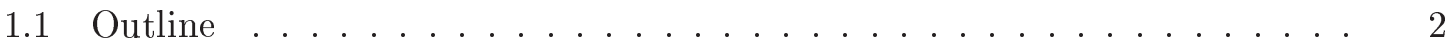

2 Theory 4

2.1 The elementary particles . . . . . . . . . . . . . . 5

2.2 Weak interactions . . . . . . . . . . . . . . . . . 7

2.2.1 The CKM matrix and $C P$ violation $\ldots \ldots \ldots . \ldots . \ldots$

2.2 .2 The unitarity triangle . . . . . . . . . . . . . . . . . 9

2.2.3 Importance of measuring $\left|V_{u b}\right| \ldots \ldots \ldots \ldots$

2.3 Semileptonic $B$-meson decays . . . . . . . . . . . . . . . . . . . 12

2.4 Exclusive measurement . . . . . . . . . . . . . . . . . . . . . . 14

2.5 Inclusive measurement f . . . . . . . . . . . . . . . . . . 15

2.5.1 Duality and the spectator model . . . . . . . . . . 15

2.5.2 Heavy Quark Expansion . . . . . . . . . . . . . . . . . . . . 16

2.5.3 Application of $\mathrm{HQE} \ldots \ldots \ldots \ldots$ 
2.5.4 Weak annihilation ................... 21

2.5.5 Perturbative corrections and $b$-quark mass . . . . . . . . . . . . 21

2.5.6 Shape Function . . . . . . . . . . . . . . . . . . . . 22

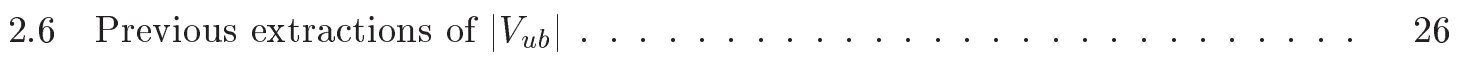

2.7 The $q^{2}-E_{\ell}^{*}$ approach $\ldots \ldots \ldots \ldots \ldots \ldots \ldots \ldots \ldots$

2.8 Comparison with other approaches . . . . . . . . . . . . . . . 29

3 The BABAR Experiment $\quad 30$

3.1 The PEP-II collider . . . . . . . . . . . . . . . . . . . 33

3.2 The tracking system $\ldots \ldots \ldots \ldots \ldots \ldots$

3.2 .1 The silicon vertex tracker . . . . . . . . . . . . 36

3.2 .2 The drift chamber . . . . . . . . . . . . . . . . 39

3.3 The Čerenkov light detector . . . . . . . . . . . . . . . . . . . . 43

3.4 The electromagnetic calorimeter . . . . . . . . . . . . . 45

3.5 The instrumented flux return . . . . . . . . . . . . . . . . 49

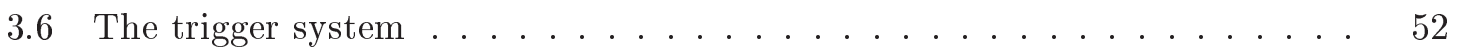

$3.6 .1 \quad$ L1 trigger . . . . . . . . . . . . . . . . . . 52

3.6 .2 L3 trigger . . . . . . . . . . . . . . . 54

4 Data and Monte Carlo Samples

4.1 On-peak and off-peak data . . . . . . . . . . . . . . . 55

4.2 Monte Carlo simulations . . . . . . . . . . . . . . . 55

4.2.1 Simulation of signal events . . . . . . . . . . . . . 56

4.2 .2 HQE parameters . . . . . . . . . . . . . . . . . 59

4.2.3 Simulation of backgrounds . . . . . . . . . . . . . . . . . . 64

5 Selection of Semileptonic Decays $\quad 66$

5.1 Analysis strategy . . . . . . . . . . . . . . . 66

5.1 .1 Continuum subtraction _................... 67 


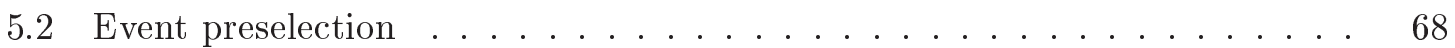

5.3 Particle selection $\ldots \ldots \ldots \ldots \ldots \ldots$

5.3.1 General philosophy . . . . . . . . . . . . . . . . . . 69

5.3 .2 Charged track selection $\ldots \ldots \ldots \ldots$

5.3.3 Neutral energy selection . . . . . . . . . . . . . . . . . . 72

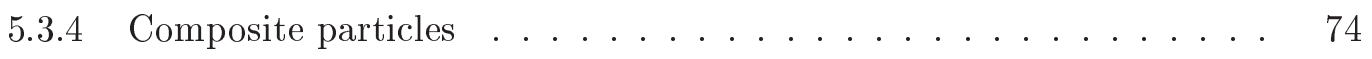

5.3 .5 Particle identification . . . . . . . . . . . . . 75

5.3.6 Validation from missing momentum reconstruction . . . . . . . . 76

5.4 Neutrino and $q^{2}$ reconstruction $\ldots \ldots \ldots \ldots \ldots$

5.5 Refinement of the event selection . . . . . . . . . . . . . . 82

5.6 Comparison between data and simulation $\ldots \ldots \ldots \ldots$

6 Control Sample Studies $\quad 95$

6.1 Selecting $B \rightarrow D^{0} e \bar{\nu}_{e}$ events $\ldots \ldots \ldots \ldots \ldots$

6.2 Adjusting background branching ratios . . . . . . . . . . . . . . 98

6.3 Study of neutrino reconstruction . . . . . . . . . . . . . 103

6.4 Additional tests . . . . . . . . . . . . . . . . . . 106

6.5 Adjusting Monte Carlo efficiencies . . . . . . . . . . . . . . . . . 108

6.6 Comparison between data and simulation . . . . . . . . . . . . . 111

7 Systematics Studies $\quad 117$

7.1 Signal extraction . . . . . . . . . . . . . . . . . . 117

7.2 Procedure for evaluating uncertainties . . . . . . . . . . . . . . . 120

7.3 Modeling of $B \rightarrow X_{u} e \bar{\nu}_{e}$ signal events . . . . . . . . . . . . . 121

7.4 Background simulation . . . . . . . . . . . . . . . . . . . . 124

7.5 Tracking efficiency . . . . . . . . . . . . . . . . . 128

7.6 Reconstruction of $K_{S}^{0} \ldots \ldots \ldots \ldots \ldots \ldots \ldots$

7.7 Electron identification $\ldots \ldots \ldots \ldots \ldots$ 


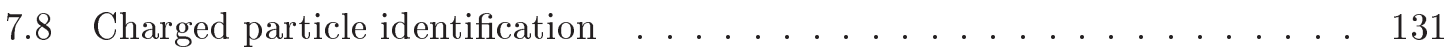

7.9 Neutrals corrections and systematics . . . . . . . . . . . . . . . 132

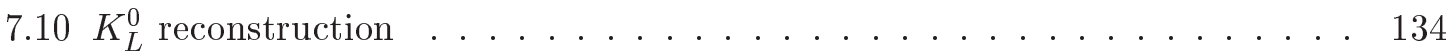

7.11 Bremsstrahlung emission . . . . . . . . . . . . . . . . . 135

7.12 Radiative corrections . . . . . . . . . . . . . . . . . . . . 138

$7.13 B$-meson counting and continuum scaling . . . . . . . . . . . . . 140

7.14 Summary of uncertainties . . . . . . . . . . . . . . . . . . 140

8 Results $\quad 142$

$8.1 \quad$ Measurements of $\mathcal{B}_{u}^{\mathrm{SL}}$ and $\Delta \mathcal{B}_{u}^{\mathrm{SL}} \ldots \ldots \ldots \ldots \ldots \ldots \ldots$

8.2 Classic extraction of $\left|V_{u b}\right| \ldots \ldots \ldots \ldots \ldots \ldots$

8.3 Improved theory and $\left|V_{u b}\right|$ extraction $\ldots \ldots \ldots \ldots \ldots \ldots$

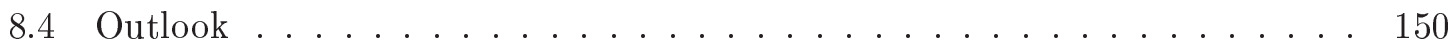

9 Conclusion $\quad 151$

$\begin{array}{lr}\text { A Defining } s_{\mathrm{h}}^{\max } & 158\end{array}$

B Particle Identification $\quad 160$

B.1 Electron identification . . . . . . . . . . . . . . . . . 160

B.2 Kaon identification . . . . . . . . . . . . . . . 161

B.3 Muon identification . . . . . . . . . . . . . . . . . . . . . . 162

B.4 Proton identification . . . . . . . . . . . . . . 163

$\begin{array}{ll}\text { C Alternative Results } & 165\end{array}$ 


\section{List of Figures}

2.1 The unitarity triangle . . . . . . . . . . . . . . . . 10

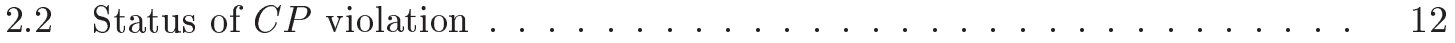

2.3 First order Feymann diagrams for $B$-meson decay . . . . . . . . . . . . 13

2.4 Kinematics of $B \rightarrow X e \bar{\nu}_{e}$ decay. . . . . . . . . . . . . . . 17

2.5 Perturbative contributions to the effective QCD Lagrangian . . . . . . . . 19

2.6 Fermi motion spectra . . . . . . . . . . . . . . . . . . . 24

2.7 Shape Function smearing effect . . . . . . . . . . . . . 25

2.8 Status of $\left|V_{u b}\right|$ measurements prior to this study . . . . . . . . . . . 27

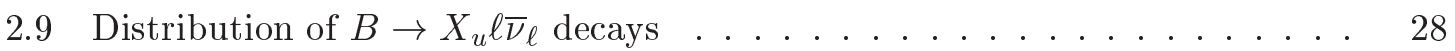

3.1 Longitudinal view of the BABAR detector . . . . . . . . . . . . . . . . 32

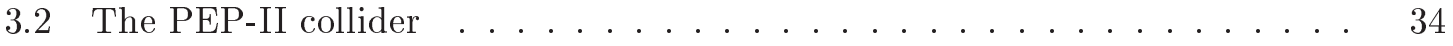

3.3 Integrated luminosity delivered by the PEP-II collider and recorded by the BABAR detector . . . . . . . . . . . . . . . . 35

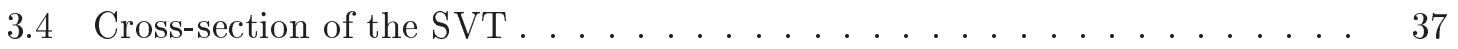

3.5 Longitudinal view of the $\mathrm{SVT} \ldots \ldots \ldots \ldots \ldots \ldots$

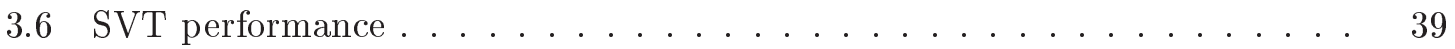

3.7 Longitudinal cross-section of the $\mathrm{DCH} \ldots \ldots \ldots$. . . . . . . . 40

3.8 Schematic layout of drift cells and drift cell isochrones . . . . . . . . . . . . 41

3.9 Particle identification via $\mathrm{dE} / \mathrm{dx}$ measurements in the $\mathrm{DCH} \ldots \ldots . . . .42$

3.10 Schematic view of the DIRC . . . . . . . . . . . . . . . 44 
3.11 Longitudinal cross-secion of the EMC . . . . . . . . . . . . . . . . 47

3.12 Schematic view of an EMC crystal assembly . . . . . . . . . . . . . 48

3.13 EMC performance . . . . . . . . . . . . . . . . . . . 49

3.14 IFR barrel and endcap wheels . . . . . . . . . . . . . . . . . 50

3.15 Cross-section of a RPC . . . . . . . . . . . . . . . . 51

3.16 Distribution of $z_{0}$ for tracks reconstructed by L3 . . . . . . . . 53

4.1 Mass distribution of $X_{u} \ldots \ldots \ldots \ldots \ldots$

$4.2 X_{u}$ mass spectrum for the hybrid sample . . . . . . . . . . . . . . 60

4.3 Electron energy spectrum for the hybrid sample . . . . . . . . . . . . . 61

$4.4 q^{2}$ spectrum for the hybrid sample $\ldots \ldots \ldots \ldots$. . . . . . . . . 62

$4.5 s_{\mathrm{h}}^{\max }$ spectrum for the hybrid sample .............. 63

5.1 Multiplicity and energy reconstruction from selected charged tracks . . . . . 73

5.2 Multiplicity and energy reconstruction from selected neutrals . . . . . . . 74

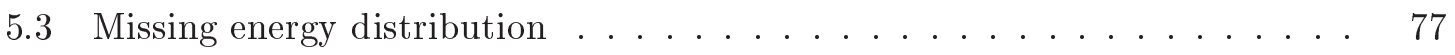

5.4 Missing momentum resolution . . . . . . . . . . . . . . . . 78

5.5 The missing invariant mass squared vs $E_{\text {miss }}^{*}-\left|\vec{P}_{\text {miss }}^{*}\right| \ldots \ldots \ldots$

5.6 Bias correction for neutrino estimator f . . . . . . . . . . . . . 81

5.7 Neutrino and $q^{2}$ resolution . . . . . . . . . . . . . . . . . . . 82

5.8 Reconstruction of $s_{\mathrm{h}}^{\max } \ldots \ldots \ldots \ldots \ldots \ldots$

5.9 Useful variables for continuum suppression . . . . . . . . . . . 85

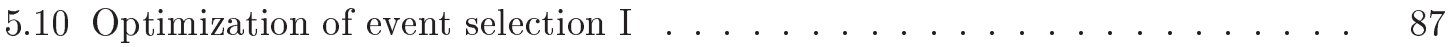

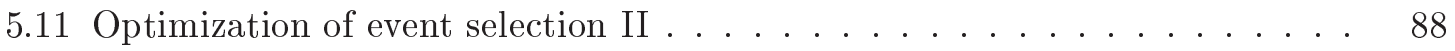

5.12 Multiplicity for data and MC after the refined event selection . . . . . . . 92

5.13 Data and MC distributions satisfying the refined event selection I . . . . . . 93

5.14 Data and MC distributions satisfying the refined event selection II _ . . . . 94

6.1 Distribution of $\cos \theta_{B, D^{0} e}$ for the control sample . . . . . . . . . 98 
6.2 Multiplicity for the $B \rightarrow D^{0} e \bar{\nu}_{e} X$ control sample . . . . . . . . . . . . . . 99

6.3 Distributions for $B \rightarrow X_{c} e \bar{\nu}_{e}$ tuning . . . . . . . . . . . . . 100

6.4 Three-dimensional distributions before and after tuning . . . . . . . . . . 101

6.5 Distributions before and after applying the $B \rightarrow X_{c} e \bar{\nu}_{e}$ tuning I . . . . . . 103

6.6 Distributions before and after applying the $B \rightarrow X_{c} e \bar{\nu}_{e}$ tuning II . . . . . 104

6.7 Resolution for the two $B \rightarrow D^{0} e \bar{\nu}_{e} X$ neutrino estimators . . . . . . . . 106

6.8 Neutrino pseudo-resolution for the $B \rightarrow D^{0} e \bar{\nu}_{e} X$ sample . . . . . . . . 107

6.9 Electron and $K_{S}^{0}$ momentum distributions in the opposite hemisphere . . . 109

6.10 Mass spectrum for the reconstructed $D^{0} \ldots \ldots \ldots \ldots$

6.11 Data and Monte Carlo distributions for the $B \rightarrow D^{0} e \bar{\nu}_{e} X$ sample I . . . . 113

6.12 Data and Monte Carlo distributions for the $B \rightarrow D^{0} e \bar{\nu}_{e} X$ sample II . . . . 114

6.13 Data and Monte Carlo distributions for the $B \rightarrow D^{0} e \bar{\nu}_{e} X$ sample III . . . 115

6.14 Data and Monte Carlo distributions for the $B \rightarrow D^{0} e \bar{\nu}_{e} X$ sample IV $\ldots 116$

7.1 Distributions of simulated events in the $q^{2}-E_{e}^{*}$ plane $\ldots \ldots \ldots \ldots$

7.2 Event multiplicity for data and Monte Carlo simulation . . . . . . . . . 124

7.3 Impact of $\mathcal{B}\left(D^{0} \rightarrow K^{0}\right.$ incl. $)$ uncertainties on reconstructed quantities . . . 126

7.4 Impact of neutral correlated uncertainty on reconstructed quantitites . . . . 133

7.5 Impact of $K_{L}^{0}$ modeling uncertainties on reconstructed quantities . . . . . . 136

7.6 Impact of uncertainties in simulating bremsstrahlung on reconstructed $E_{e}^{*}$. 138

8.1 Measurements of $\mathcal{B}_{u}^{\mathrm{SL}}$ as a function of $E_{e}^{*}$ and $s_{\mathrm{h}}^{\max } \ldots \ldots \ldots \ldots$

8.2 Binned measurements of $\mathcal{B}_{u}^{\mathrm{SL}}$ as a function of $E_{e}^{*} \ldots \ldots \ldots \ldots$

8.3 Measurements of $\mathcal{B}_{u}^{\mathrm{SL}}$ as a function of kinematic constraints . . . . . . . . 146

8.4 Updated comparison between inclusive $\left|V_{u b}\right|$ determinations . . . . . . . . 149

B.1 Čerenkov angle measurement and particle identification . . . . . . . . . 162 


\section{List of Tables}

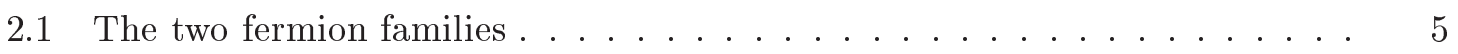

2.2 The vector gauge bosons $\ldots \ldots \ldots \ldots \ldots \ldots$

2.3 Current average measurements of the CKM parameters . . . . . . . . . 11

2.4 Theoretical results for obtaining $\left|V_{u b}\right|$ from $\mathcal{B}\left(B \rightarrow X_{u} e \bar{\nu}_{e}\right) \ldots \ldots \ldots \ldots$

3.1 Cross-section for $e^{+} e^{-}$collisions at $\sqrt{s}=10.58 \mathrm{GeV} \ldots \ldots \ldots \ldots$

3.2 Properties of $\mathrm{CsI}(\mathrm{Tl}) \ldots \ldots \ldots \ldots \ldots \ldots$

4.1 Data and Monte Carlo samples used in this analysis . . . . . . . . . 56

4.2 Composition of the signal $B \rightarrow X_{u} e \bar{\nu}_{e}$ hybrid model $\ldots \ldots \ldots$. . . . . 57

4.3 Phase-space segmentation of the signal $B \rightarrow X_{u} e \bar{\nu}_{e}$ hybrid model . . . . . 59

4.4 Branching fraction for semileptonic $B$ decays to $D$ meson $\ldots \ldots \ldots \ldots$

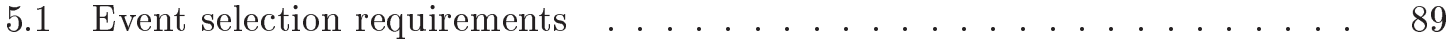

5.2 Residual yields after successive requirements for $B^{0}$ decays $\ldots \ldots$. . . . 89

5.3 Residual yields after successive requirements for $B^{+}$decays $\ldots \ldots$. . . . 90

5.4 Data and Monte Carlo yields satisfying the event requirements . . . . . . 91

6.1 Adjusted $B \rightarrow X_{c} e \bar{\nu}_{e}$ backround decay modes . . . . . . . . . . . . . . 102

6.2 Selection efficiencies for the $B \rightarrow D^{0} e \bar{\nu}_{e} X$ control sample . . . . . . . 111

$7.1 \quad$ Variables entering $\mathcal{B}_{u}^{\mathrm{SL}}$ and $\Delta \mathcal{B}_{u}^{\mathrm{SL}}$ extraction $\ldots \ldots \ldots \ldots \ldots \ldots$

7.2 Yields and efficiencies using nominal branching ratios . . . . . . . . . . . 121 
7.3 Systematic uncertainties from $B \rightarrow X_{u} e \bar{\nu}_{e}$ modeling . . . . . . . . . . . . 123

7.4 Branching ratio of various charm decay modes and uncertainties . . . . . 125

7.5 Systematic uncertainties due to background modeling . . . . . . . . . . . 127

7.6 Systematics uncertainties from detector modeling . . . . . . . . . . . . . . 129

7.7 Correction factor for $K_{L}^{0}$ energy deposition . . . . . . . . . . . . 135

7.8 Final state radiation uncertainty and resulting $E_{e}^{*}$ shift . . . . . . . . . 139

7.9 Summary of systematic uncertainties . . . . . . . . . . . . . . . 141

8.1 Yields and efficiencies using nominal branching ratios . . . . . . . . . . . . . 142

8.2 Partial theory rates for various $\tilde{E}_{e}$ requirements . . . . . . . . . . . 148

C.1 Yields and efficiencies for $E_{e}^{*}>2.0 \mathrm{GeV} \ldots \ldots \ldots \ldots$. . . . . . 165

C.2 Yields and efficiencies for $E_{e}^{*}>2.2 \mathrm{GeV} \ldots \ldots \ldots \ldots$. . . . . . 166

C.3 Summary of systematic uncertainties for $E_{e}^{*}>2.0 \mathrm{GeV} \ldots \ldots \ldots 76$

C.4 Summary of systematic uncertainties for $E_{e}^{*}>2.2 \mathrm{GeV} \ldots \ldots \ldots$ 


\section{Chapter 1}

\section{Introduction}

Felix qui potuit rerum cognoscere causas

-Virgil

One of the outstanding questions in cosmology pertains to the disappearance of antimatter from the Universe. In modern theories of cosmology the net baryon number of the early Universe is zero. Today it is clearly not the case, at least in our local region. Three necessary conditions for generating such excess of matter in the evolution of the Universe have been identified: thermal imbalance, baryon number violation and $C P$ violation [1].

The simultaneous violation of Charge conjugation $(C)$ and Parity-symmetry $(P)$ was first observed in neutral kaon decays in 1964 [2]. It is an expected consequence of the Standard Model (SM) of particle physics, and arises from the complex phase of the Cabbibo-Kobayashi-Maskawa Matrix (CKM) which describes the weak couplings between quarks. Calculations based on the SM show that this phase cannot produce enough matterantimatter imbalance to produce the current asymmetry [3]. Hence, a thorough experimental investigation of $C P$ violation is needed to test the model predictions and search for sources of $C P$ violation beyond the SM.

A plethora of $C P$-violation effects are expected in $B$-meson decays, some of which are well predicted by the Standard Model. Hence, measurements of $B$ mesons decaying into $C P$ eigenstates can provide a series of unique consistency tests of the quark sector of the SM. The $B A B A R$ experiment was designed to study the millions of $B \bar{B}^{1}$ pairs produced by

\footnotetext{
${ }^{1} B$ and anti- $B$ mesons; mesons which contain an anti-bottom or bottom quark, respectively.
} 
the PEP-II $e^{+} e^{-}$collider at the Stanford Linear Accelerator Center [4]. The increasingly precise measurements of $C P$ asymmetries performed by $B A B A R$ allow stringent experimental tests of the SM mechanism for $C P$ violation [5]. Improved determinations of the CKM element $\left|V_{u b}\right|$, the weak coupling of the bottom quark to the up quark, directly improve the sensitivity of these experimental tests.

From an experimental and theoretical point of view, inclusive charmless semileptonic $B$ decays, $B \rightarrow X_{u} e \bar{\nu}_{e}$, are the most accessible channel for measuring $\left|V_{u b}\right|$. Two observables have been used to determine $\left|V_{u b}\right|$ from inclusive semileptonic $B$ decays: the endpoint of the lepton momentum spectrum [6] and the mass of the accompanying hadronic system [7]. In this dissertation, $B \rightarrow X_{u} e \bar{\nu}_{e}$ decays are identified from a sample of 88 million $B \bar{B}$ pairs collected by the BABAR detectorusing a novel approach based on simultaneous requirements for the electron energy, $E_{e}$, and the invariant mass squared of the $e \bar{\nu}_{e}$ pair, $q^{2}$ [8]. The neutrino 4-momentum is reconstructed from the visible 4-momentum and knowledge of the $e^{+} e^{-}$initial state. The dominant charm background is suppressed by selecting a region of the $q^{2}-E_{e}$ phase space where correctly reconstructed $B \rightarrow X_{c} e \bar{\nu}_{e}$ events are kinematically excluded. Background contamination in the signal region is due to resolution effects and is evaluated in Monte Carlo simulations. Further selection criteria are imposed to ensure a proper neutrino reconstruction. The total and partial $B \rightarrow X_{u} e \bar{\nu}_{e}$ branching ratio is then measured using the observed yield in data, and background estimates and signal efficiencies evaluated in Monte Carlo simulations. Finally, theoretical calculations based on the Heavy Quark Expansion, an effective field theory, allow for the determination of the CKM element $\left|V_{u b}\right|$ using the measured $B$-meson lifetime and $B \rightarrow X_{u} e \bar{\nu}_{e}$ branching ratio. The determination of $\left|V_{u b}\right|$ is limited mostly by our knowledge of the $b$-quark mass.

\subsection{Outline}

A brief overview of the Standard Model of particle physics is made in chapter 2. In particular, the relationship between the element $\left|V_{u b}\right|$ of the CKM matrix and $C P$ violation 
is discussed, and the theory of semileptonic $B$ decays is developed in the framework of the Heavy Quark Expansion into an expression for $\left|V_{u b}\right|$ in terms of the $B$-meson lifetime and $B \rightarrow X_{u} e \bar{\nu}_{e}$ branching ratio. In chapter 3 , the experimental environment is presented. The data and details of the Monte Carlo simulation samples used in this study are described in chapter 4 . In chapter 5 , the analysis strategy is outlined, and the remainder of chapter 5 to chapter 7 describe the various steps leading to the extraction of the $B \rightarrow X_{u} e \bar{\nu}_{e}$ branching ratio and the evaluation of the dominant systematic uncertainties. Results are presented in chapter 8, where values for the CKM matrix element $\left|V_{u b}\right|$ are extracted. 


\title{
Chapter 2
}

\section{Theory}

\author{
Ex nihilo nihil \\ -Lucrece
}

Elementary particle physics aims at the understanding of the fundamental constituents of matter and the forces between them. Its roots may be traced back to 1897 with the discovery of the electron by J.J. Thomson. It was already known at the time that cathode rays carried an electric charge since they could be deflected by a magnet. This suggested that these rays were in fact charged particles. Thomson set up an experiment in which a cathodic beam was sent through crossed electric and magnetic field. By tuning the fields strength until the net deflection was zero, he was able to determine the velocity of the particles as well as the ratio of their mass over their charge. He named these particles corpuscles, and their charge the electron.

Over the last one hundred years, particle physics has tremendously evolved. Three theories were united in what is now referred to as the Standard Model (SM). It describes all of the known fundamental particles and their interactions via three forces: electromagnetism, the weak and the strong forces. Quantum Electrodynamics (QED) was the first theory developed and describes electromagnetic phenomena. Later, in the late 1960's, the weak and electromagnetic interactions were unified in the theory of electroweak interactions proposed by Glashow, Weinberg and Salam [9, 10, 11]. The Glashow-Weinberg-Salam theory incorporates QED processes and provides a description of the weak force. The third theory, Quantum Chromodynamics (QCD), describes the interactions of quarks through 


\begin{tabular}{|c|c|c|c|c|}
\hline Families & charge & 1st generation & 2nd generation & 3rd generation \\
\hline \hline Quarks & $+2 / 3$ & $u$ & $c$ & $t$ \\
& $-1 / 3$ & $d$ & $s$ & $b$ \\
\hline \multirow{2}{*}{ Leptons } & -1 & $e$ & $\mu$ & $\tau$ \\
& 0 & $\nu_{e}$ & $\nu_{\mu}$ & $\nu_{\tau}$ \\
\hline
\end{tabular}

Table 2.1: The two fermion families.

the strong "colour" field. In the last thirty years, the SM has been intensively tested by numerous experiments; so far, no experimental result has been conclusively shown to disagree with the predictions of the SM.

In the following sections, an overview of the Standard Model elementary particles and their interactions will be given, followed by a description of the CKM mechanism for charged electroweak interactions. The relationship between $C P$ violation and the CKM matrix will then be discussed, and the importance of obtaining a precise measurement of the element $\left|V_{u b}\right|$ of the matrix, the goal of this dissertation, will be highlighted. Finally, theoretical predictions for charmless semileptonic $B$ decays will be developed into an expression for extracting $\left|V_{u b}\right|$ from a measurement of the inclusive $B \rightarrow X_{u} \ell \bar{\nu}_{\ell}$ branching ratio.

\subsection{The elementary particles}

The Standard Model is composed of three types of particles: the spin- $1 / 2$ fermions (leptons and quarks) that constitute all matter, the spin-1 gauge bosons that mediate the forces through which particles interact, and the spin-0 Higgs boson. As shown in table 2.1, there are in total 12 fermions grouped in two families, each of which is divided into three generations. The electron $(e)$, muon $(\mu)$ and tau $(\tau)$ are charged leptons, whereas all of their neutrino partners $\left(\nu_{e}, \nu_{\mu}\right.$ and $\left.\nu_{\tau}\right)$ are electrically neutral.

The quark family is composed of the up $(u)$, charm $(c)$ and top $(t)$ quarks which carry a charge of $+\frac{2}{3} e$ each, whereas the down $(d)$, strange $(s)$, and bottom $(b)$ quarks have an electrical charge of $-\frac{1}{3} e$. Furthermore, for each fermion there exists an anti-fermion of 
equal mass but with opposite charge and flavour number. For instance, the positron $\left(e^{+}\right)$ is the anti-particle partner of the electron $\left(e^{-}\right)$.

Three of the four known forces are part of the SM: the strong, electromagnetic, and weak forces; gravity is by far the weakest force and is not part of the Standard Model. The electroweak force acts on both leptons and quarks via the exchange of vector gauge bosons: $\gamma, W^{+}, W^{-}$or $Z^{0}$. The unification of the electromagnetic and weak interactions to the electroweak interaction is an essential part of the SM, and requires the existence of at least one Higgs boson. The masses of fermions and gauge bosons are generated after spontaneous symmetry breaking of the vacuum via the Higgs mechanism producing a massive neutral scalar Higgs boson $[10,11]$. With the discovery of the $W^{ \pm}[12]$ and $Z^{0}[13]$ at $\mathrm{CERN}^{1}$ in 1983, and of the top quark in 1995 at Fermilab [14], the Higgs boson is the only remaining particle of the Standard Model which has not been experimentally observed. The discovery of the Higgs boson is the goal of two experiments planned at the Large Hadron Collider, which is currently under construction at CERN.

Leptons do not feel the strong force which acts only on quarks that carry one of three "colour" charges. The strong force is mediated by the exchange of coloured gluons $(g)$, which bind quarks to produce colourless particles called hadrons. Each of the six quarks comes in three different colours, denoted as "red", "blue" and "green". Quarks can therefore combine in two different ways to produce a colourless state. A quark (red, blue or green) can combine with an antiquark (antired, antiblue, or antigreen) to produce integer spin particles called mesons, such as the $\pi^{+}(u \bar{d})$ and the $\pi^{-}(\bar{u} d)$. It is also possible for three quarks (red, blue and green) or three antiquarks (antired, antiblue and antigreen) to combine and form a half-integer spin particle called a baryon such as the proton (uud) and neutron $(u d d)$. A summary of the vector bosons and the forces they mediate is presented in table 2.2 .

\footnotetext{
${ }^{1}$ Conseil Européen pour la Recherche Nucléaire, now the European Center for Particle Physics.
} 


\begin{tabular}{|c|c|}
\hline Bosons & Force mediated \\
\hline \hline$\gamma$ & Electromagnetic \\
$Z^{0}$ & Neutral weak \\
$W^{ \pm}$ & Charged weak \\
$g$ & Strong \\
\hline
\end{tabular}

Table 2.2: The vector gauge bosons.

\subsection{Weak interactions}

Neutral and charged weak interactions are mediated through the exchange of massive vector bosons: the $\mathrm{Z}^{0}$ and the $\mathrm{W}^{ \pm}$, respectively. In the neutral current, quark flavour is conserved. On the other hand, quark flavour changes in charged current processes such as the decay of a bottom quark to an up quark and a lepton anti-neutrino pair:

$$
b \rightarrow W^{*-} u, W^{*-} \rightarrow \ell \overline{\nu_{\ell}},
$$

where $W^{*-}$ denotes a virtual $W^{-}$boson. At tree level, ${ }^{2}$ this weak decay transition is described by the Lagrangian

$$
\mathcal{L}=-\frac{g}{\sqrt{2}} \mathcal{J}^{\mu} W_{\mu}^{+}+\text {h.c. },
$$

where $\mathcal{J}^{\mu}$ is a V-A charged weak current operator coupling to the $W$ boson,

$$
\mathcal{J}^{\mu}=\left(\bar{\nu}_{e}, \bar{\nu}_{\mu}, \bar{\nu}_{\tau}\right) \gamma^{\mu}\left(1-\gamma^{5}\right)\left(\begin{array}{c}
e^{-} \\
\mu^{-} \\
\tau^{-}
\end{array}\right)+(\bar{u}, \bar{c}, \bar{t}) \gamma^{\mu}\left(1-\gamma^{5}\right) V^{\mathrm{CKM}}\left(\begin{array}{c}
d \\
s \\
b
\end{array}\right) .
$$

The first term allows transitions between a charged lepton and its corresponding flavour neutrino, and is governed by a universal coupling constant, $g_{w}$, often expressed as

$$
g_{w}=\frac{e}{\sin \theta_{w}}
$$

where $\theta_{w}$ is the weak or Weinberg mixing angle and $e$ is the electromagnetic charge. At low energy, the charged weak-current interaction gives rise to local four-fermion couplings of the form

$$
\mathcal{L}_{\text {eff }}=-2 \sqrt{2} G_{F} \mathcal{J}^{\mu} \mathcal{J}_{\mu}^{\dagger},
$$

\footnotetext{
${ }^{2}$ The lowest order in a perturbation expansion.
} 
where $G_{F}$ is the Fermi constant $G_{F}=\sqrt{2} g_{w}^{2} / 8 M_{W}^{2}$. The matrix $V^{\mathrm{CKM}}$ in the second term of equation 2.3 is known as the Cabbibo-Kobayashi-Maskawa (CKM) matrix, and relates weak interaction eigenstates to quark flavour eigenstates, and can be written explicitly as

$$
V^{\mathrm{CKM}}=\left(\begin{array}{ccc}
V_{u d} & V_{u s} & V_{u b} \\
V_{c d} & V_{c s} & V_{c b} \\
V_{t d} & V_{t s} & V_{t b}
\end{array}\right) .
$$

Therefore, transitions between two quarks $q, Q$ are possible even between different generations. The relative size of the charged current amplitude is then proportional to $\left|V_{q Q}\right|$. In this dissertation, the magnitude of the element $\left|V_{u b}\right|$ of the matrix will be determined using the process outline in equation 2.1.

\subsubsection{The CKM matrix and $C P$ violation}

In quantum field theory, the Lagrangian is invariant under three operations: time reversal, parity, and charge conjugation. Under time reversal $(T)$,

$$
T \psi(t, \vec{x}) \rightarrow \psi(-t, \vec{x})
$$

The parity operator $(P)$ reverses the handedness of space such that

$$
P \psi(t, \vec{x}) \rightarrow \psi(t,-\vec{x}) .
$$

In other words, a symmetry through the origin of the reference system can be found, leaving the spin the same. Finally, charge conjugation $(C)$ is the operation which replaces all particles by their antiparticles, but where all space-time properties remain the same. Hence, the combination $C P$ replaces a particle by its antiparticle and reverses momentum and helicity. ${ }^{3}$ The strong and electromagnetic interactions have been shown experimentally to respect $C, P$ and $T$ symmetries, whereas weak interactions have been observed to violate both $C$ and $P$. Although $C P$ violation has been observed in neutral kaon [2] and $B$ meson decays [15], no experimental results have shown disagreement with exact $C P T$.

\footnotetext{
${ }^{3}$ The direction of the spin with respect to the particule momentum.
} 
Kobayashi and Maskawa realized that a $3 \times 3$ matrix has an irreducible complex phase from which $C P$ violation observed in neutral kaon decays can, in principle, be explained [5]. This is what led them to propose a third generation of quarks even before the charm quark was discovered. Nowadays, the "standard" parameterization of the CKM matrix is expressed in terms of this complex phase and three real angles:

$$
V^{\mathrm{CKM}}=\left(\begin{array}{ccc}
c_{12} c_{13} & s_{12} c_{13} & s_{13} e^{-i \delta_{13}} \\
-s_{12} c_{23}-c_{12} s_{23} s_{13} e^{i \delta_{13}} & c_{12} c_{23}-s_{12} s_{23} s_{13} e^{i \delta_{13}} & s_{23} c_{13} \\
s_{12} s_{23}-c_{12} c_{23} s_{13} e^{i \delta_{13}} & -c_{12} s_{23}-s_{12} c_{23} s_{13} e^{i \delta_{13}} & c_{23} c_{13}
\end{array}\right),
$$

where $\delta_{13}$ is the complex phase, and $c_{i j}=\cos \theta_{i j}$ and $s_{i j}=\sin \theta_{i j}$ for the 3 generations $i, j=1,2,3[16]$. Experiments have shown that $s_{12} \gg s_{23} \gg s_{13}$, such that equation 2.9 may be expanded in terms of powers of $s_{12}$,

$$
V^{\mathrm{CKM}}=\left(\begin{array}{ccc}
1-\frac{\lambda^{2}}{2} & \lambda & A \lambda^{3}(\rho-i \eta) \\
-\lambda & 1-\frac{\lambda^{2}}{2} & A \lambda^{2} \\
A \lambda^{3}(1-\rho-i \eta) & -A \lambda^{2} & 1
\end{array}\right)+\mathcal{O}\left(\lambda^{4}\right),
$$

where $s_{12}=\lambda \approx V_{u s}, s_{23}=A \lambda^{2} \approx V_{c b}$, and $s_{13} e^{-i \delta_{13}}=A \lambda^{3}(\rho-i \eta)=V_{u b}$ [17]. Note that written this way, all four parameters $(A, \lambda, \rho, \eta)$ are real. $C P$ violation may then be accounted fully by the CKM mechanism if $\eta \neq 0$ and $\eta \neq \pi$. One of the goals of BABAR is to test whether this model is valid.

\subsubsection{The unitarity triangle}

The unitarity of the CKM matrix, $V V^{\dagger}=I$, implies various relationships amongst its elements. Three of them are very useful in understanding the Standard Model predictions for $C P$ violation:

$$
\begin{aligned}
& V_{u d} V_{u s}^{*}+V_{c d} V_{c s}^{*}+V_{t d} V_{t s}^{*}=0, \\
& V_{u s} V_{u b}^{*}+V_{c s} V_{c b}^{*}+V_{t s} V_{t b}^{*}=0, \\
& V_{u d} V_{u b}^{*}+V_{c d} V_{c b}^{*}+V_{t d} V_{t b}^{*}=0 .
\end{aligned}
$$

Each of these three relations requires the sum of three complex quantities to vanish and may be represented graphically in the complex plane as a triangle. The third expression in equation 2.11 is of particular interest as it is related to $B$ physics as studied in BABAR and 


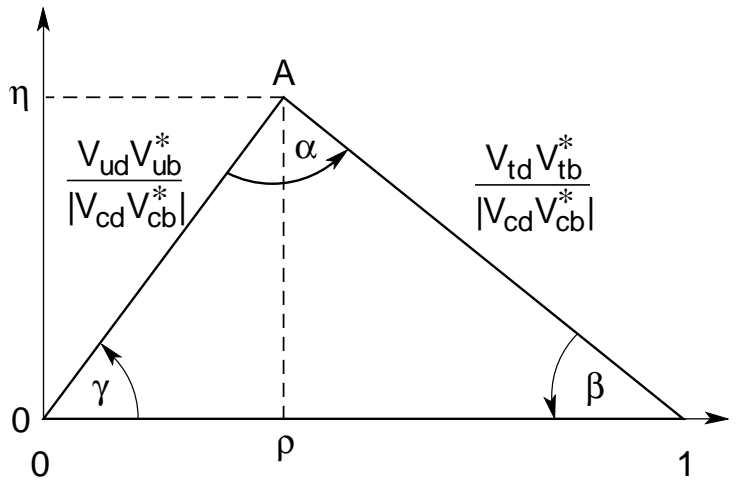

Figure 2.1: The rescaled unitarity triangle. The phase convention is such that $V_{c d} V_{c b}^{*}$ is real.

will be referred to as "the unitarity triangle" throughout this dissertation. Figure 2.1 depicts this particular triangle. The phase convention chosen is such that $V_{c d} V_{c b}^{*}$ is real. It is then possible to normalize the triangle to the length of $\left|V_{c d} V_{c b}^{*}\right|$. The coordinates of the remaining vertex, where $V_{u d} V_{u b}^{*}$ and $V_{t d} V_{t b}^{*}$ meet, are denoted by the Wolfenstein parameters $\rho$ and $\eta$, the later quantity, which dictates the openness of the triangle, reflects the amount of $C P$ asymmetries to expect. In fact, if there was no $C P$ violation, the imaginary phase $\eta$ would be zero such that $\alpha=\beta=\gamma=0$, i.e, the triangle would collapse into a flat line. The same procedure may also be applied for the leading $K^{0}$ and $B_{s}$ decays, which correspond to the first and second expression of equation 2.11, respectively. But measurements of the $\left|V_{q Q}\right|$ yield one side of these being much shorter than the other two, such that these triangles almost collapse to flat lines. This explains the small $C P$ violation observed in neutral kaon decays.

By defining $\bar{\rho}=\left(1-\lambda^{2} / 2\right) \rho$ and $\bar{\eta}=\left(1-\lambda^{2} / 2\right) \eta$, one can express the sides of the unitarity triangle as

$$
\frac{V_{u d} V_{u b}^{*}}{V_{c d} V_{c b}^{*}} \approx-\sqrt{\bar{\rho}^{2}+\bar{\eta}^{2}} e^{i \gamma} \text { and } \frac{V_{t d} V_{t b}^{*}}{V_{c d} V_{c b}^{*}} \approx-\sqrt{(1-\bar{\rho})^{2}+\bar{\eta}^{2}} e^{i \beta} .
$$

The lengths of the two complex sides of the unitarity triangle in the $(\bar{\rho}, \bar{\eta})$ plane are then

$$
R_{\beta}=\sqrt{\bar{\rho}^{2}+\bar{\eta}^{2}}=\frac{1-\lambda^{2} / 2}{\lambda}\left|\frac{V_{u b}}{V_{c b}}\right|
$$




\begin{tabular}{|c|c|}
\hline Parameter & Amplitude \\
\hline \hline$V_{u d}$ & $0.9738 \pm 0.0005$ \\
\hline$V_{u s}$ & $0.2196 \pm 0.0023$ \\
\hline$V_{u b}$ & $0.00367 \pm 0.00047$ \\
\hline$V_{c d}$ & $0.224 \pm 0.012$ \\
\hline$V_{c s}$ & $0.996 \pm 0.013$ \\
\hline$V_{c b}$ & $0.0413 \pm 0.0015$ \\
\hline
\end{tabular}

Table 2.3: Current world average measurements of the CKM parameters as reported by [18]. Measurements performed by the CDF and D $\varnothing$ experiments have set crude limits on the CKM elements involving the top quark of $\frac{\left|V_{t b}\right|^{2}}{\left|V_{t d}\right|^{2}+\left|V_{t s}\right|^{2}+\left|V_{t b}\right|^{2}}=0.94_{-0.24}^{+0.31}$ and $\left|V_{t b}\right|>0.07$.

and

$$
R_{\gamma}=\sqrt{(1-\bar{\rho})^{2}+\bar{\eta}^{2}}=\frac{1}{\lambda}\left|\frac{V_{t d}}{V_{c b}}\right|,
$$

where the subscripts $\beta$ and $\gamma$ denote the angle opposite to that side. Table 2.3 summarizes the latest measurement of the CKM parameters as reported by [18]. From these values, one notices that the length $R_{\beta}$ is accessible, whereas the current status on top quarks results in a looser bound on $R_{\gamma} \propto\left|V_{t d} V_{t b}^{*}\right|=0.0083 \pm 0.0016$

The same exercise may be applied to the angles $\alpha$ and $\beta$ of the triangles such that

$$
\sin 2 \alpha=\frac{2 \bar{\eta}\left[\bar{\eta}^{2}+\bar{\rho}(\bar{\rho}-1)\right]}{\left[\bar{\eta}^{2}+(1-\bar{\rho})^{2}\right]\left[\bar{\eta}^{2}+\bar{\rho}^{2}\right]} \quad \text { and } \quad \sin 2 \beta=\frac{2 \bar{\eta}(1-\bar{\rho})}{\bar{\eta}^{2}+(1-\bar{\rho})^{2}}
$$

This is the parameterization used in BABAR. $C P$ violation in the $B$ meson system is evaluated by measuring the angles $\alpha$ and $\beta$ through specific decay channels. The Standard Model description of $C P$ violation can then be tested by looking at the agreement or non-agreement between the length $R_{\beta}$ of the unitarity triangle and the angle $\beta$ as will be discussed in the following section.

\subsubsection{Importance of measuring $\left|V_{u b}\right|$}

Recent measurements of $\sin 2 \beta$ by the $B A B A R$ and Belle experiments yield an average value of $\sin 2 \beta=0.725 \pm 0.037$ [19]. Figure 2.2 displays the possible representation of this measurement in the complex plane with $\pm 1 \sigma$ and $\pm 2 \sigma$ envelopes. Other constraints are also 


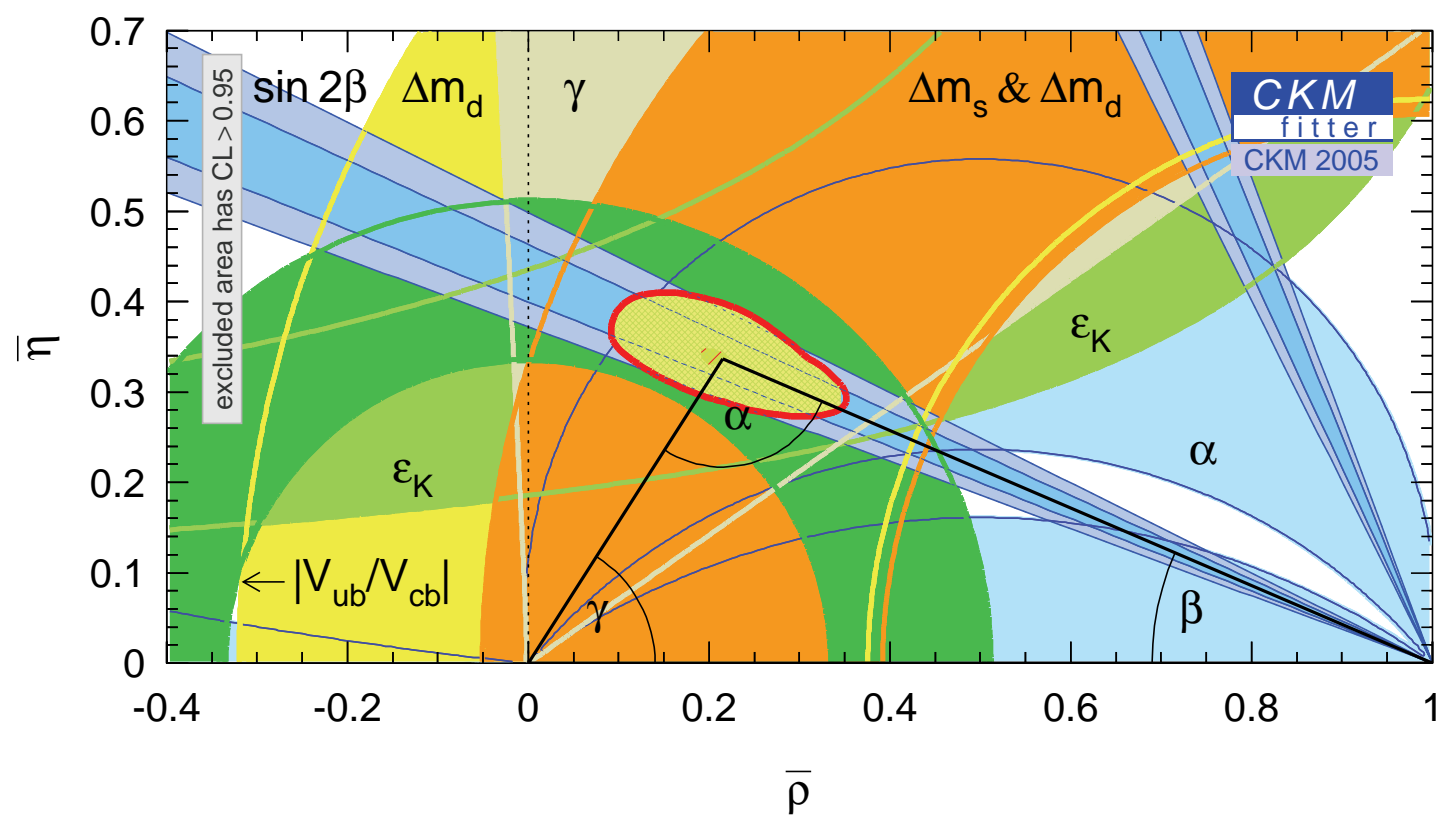

Figure 2.2: Status of $C P$ violation with respect to the Standard Model [20]. The unknown apex $(\bar{\rho}, \bar{\eta})$ of the unitarity triangle is located in the ellipse-like shaded area. It is primarily constrained by the measurements of $\sin 2 \beta$ and $\left|V_{u b} / V_{c b}\right|$.

displayed, such as the $C P$-violating parameter $\left|\epsilon_{K}\right|$ in the neutral kaon system, and $\left|V_{u b} / V_{c b}\right|$, which determines the length $R_{\beta}$ of the unitarity triangle. The $(\bar{\rho}, \bar{\eta})$ apex is then located in the region contained by the uncertainty on $\sin 2 \beta$ and $R_{\beta}$. At present, the error on $\left|V_{u b}\right|$ is about $13 \%$ (see table 2.3 ), which dominates the uncertainty $R_{\beta}$ displayed as the wide $\pm 2 \sigma$ circular region in figure 2.2 . In order to further constrain the unitary triangle and test the SM mechanism for $C P$ violation, the precision on $\left|V_{u b}\right|$ must be improved. Finally, by measuring $\left|V_{u b}\right|$, one has the opportunity to test the CKM model of weak interactions itself.

\section{$2.3 \quad$ Semileptonic $B$-meson decays}

Different types of $B$-meson decay may lead to an extraction of $\left|V_{u b}\right|$. Figure 2.3 shows the first order Feynman diagrams for the weak decay of a $B$ meson via semileptonic, hadronic and purely leptonic channels. Some of these processes are harder to select experimentally 
a)

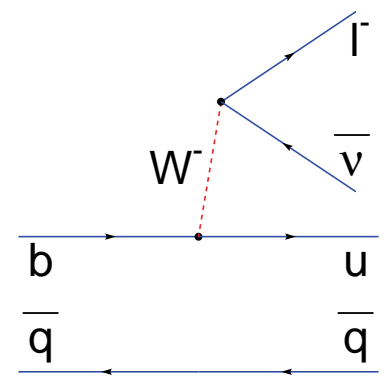

c)

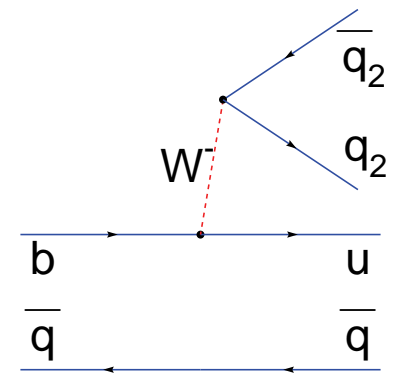

b)

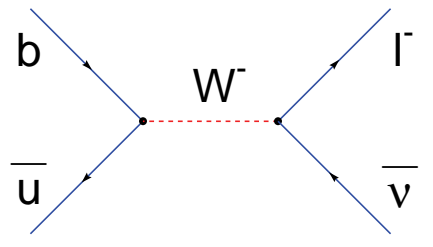

d)

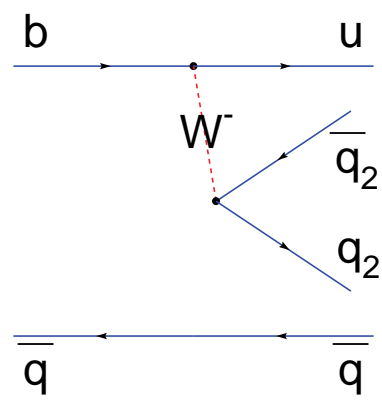

Figure 2.3: First order Feymann diagrams for the weak decay of a $B$ meson $(b \bar{q})$ via different channels: a) semileptonic, b) leptonic, and c) and d) hadronic. The label $q$ denotes a quark, $l$ a lepton and $\nu$ a neutrino.

whereas others have large theoretical uncertainties associated to them. For instance, the weak interactions underlying the hadronic processes (c and $\mathbf{d}$ ) are straightforward to describe theoretically, but complications arise because the quarks are bound inside the hadrons by the strong force. Furthermore, hadrons in the final state can interact strongly with each other. These interactions are impossible to predict using perturbative methods because the strong coupling is large at the typical energies in these decays [21].

Semileptonic and leptonic decays (figure $2.3 \mathbf{a}$ and $2.3 \mathbf{b}$, respectively) are relatively much simpler to deal with since the effects of the strong interaction can be isolated. In heavy quark decays, semileptonic modes are generally much more accessible experimentally 
simply because their branching fractions are larger.

In the following sections, the physics of charmless semileptonic B-meson decay

$$
B \rightarrow X_{u} \ell \bar{\nu}_{\ell}
$$

where $\ell$ is either an electron or muon ${ }^{4}$ and $X_{u}$ denotes any meson containing an up-quark, is developed into an expression where $\left|V_{u b}\right|$ can be extracted. Although strong interactions are difficult to calculate, they are isolated to the hadronic current in semileptonic decays. As a consequence, the effects of the strong interactions may be parameterized in terms of form factors or of a shape function as will be described in sections 2.4 and 2.5.6, respectively. The extraction of $\left|V_{u b}\right|$ is further complicated by the large background from $B \rightarrow X_{c} \ell \bar{\nu}_{\ell}$, which has a rate about 100 times higher than that of charmless semileptonic decay. Therefore, stringent kinematical constraints must be applied in order to differentiate between signal and background as will be discussed in 2.5.6. In order to improve existing determinations of $\left|V_{u b}\right|$, a compromise between experimental and theoretical uncertainties must be considered. Several methods have been used in the the past, but this work uses a novel approach which consists of measuring the invariant-mass squared of the lepton-neutrino system and the lepton energy described in section 2.7.

\subsection{Exclusive measurement}

To perform an exclusive measurement of $\left|V_{u b}\right|$, one selects decays where $X_{u}$ is a particular hadronic final state such as a pion. The $B^{0} \rightarrow \pi^{-} \ell^{+} \nu_{\ell}$ branching fraction is defined as

$$
\mathcal{B}\left(B^{0} \rightarrow \pi^{-} \ell^{+} \nu_{\ell}\right)=\frac{\Gamma\left(B^{0} \rightarrow \pi^{-} \ell^{+} \nu_{\ell}\right)}{\Gamma\left(B^{0} \rightarrow \text { anything }\right)}=\Gamma\left(B^{0} \rightarrow \pi^{-} \ell^{+} \nu_{\ell}\right) \tau_{B}
$$

where $\tau_{B}$ is the $B$-meson lifetime. The amplitude of this decay is

$$
\mathcal{M}\left(B^{0} \rightarrow \pi^{-} \ell^{+} \nu_{\ell}\right)=i \frac{G_{F}}{\sqrt{2}}\left|V_{u b}\right|\left[\bar{\psi}_{\ell} \gamma_{\mu}\left(1-\gamma^{5}\right) \psi_{\nu}\right] F^{\mu}
$$

\footnotetext{
${ }^{4}$ Because the tau lepton is both harder to reconstruct and kinematically suppressed, it is ignored in inclusive calculations.
} 
where $F^{\mu}=f_{+}\left(q^{2}\right) p^{\mu}$ is the form factor describing the $B^{0} \rightarrow W^{*} \pi^{-}$process and includes the underlying strong interactions. In the limit of a massless charged lepton, the differential decay rate can be evaluated in terms of the form factor as

$$
\frac{\mathrm{d} \Gamma\left(B^{0} \rightarrow \pi^{-} \ell^{+} \nu_{\ell}\right)}{\mathrm{d} q^{2}}=\frac{G_{F}^{2}\left|V_{u b}\right|^{2}}{24 \pi^{3}}\left|f_{+}\left(q^{2}\right)\right|^{2}\left|\vec{p}_{\pi}\right|^{3}
$$

where $q^{2}$ is the invariant mass squared of the charged lepton-neutrino system. Note that for vector meson final states such as $\rho$ and $\omega$, three form factors are necessary [22, 23]. Theoretical predictions for the normalized rate, $\tilde{\Gamma}=\Gamma /\left|V_{u b}\right|^{2}$, may then be used in conjunction with measurements of the branching ratio to extract $\left|V_{u b}\right|$ as

$$
\left|V_{u b}\right|=\sqrt{\frac{\mathcal{B}\left(B^{0} \rightarrow \pi^{-} \ell^{+} \nu_{\ell}\right)}{\tilde{\Gamma}\left(B^{0} \rightarrow \pi^{-} \ell^{+} \nu_{\ell}\right) \tau_{B}}} .
$$

Thus, it is possible to extract $\left|V_{u b}\right|$ by combining measurements of the $B^{0} \rightarrow \pi^{-} \ell^{+} \nu_{\ell}$ branching ratio and $B^{0}$-meson lifetime. Unfortunately, current form-factor evaluations result in large uncertainties in the computation of $\tilde{\Gamma}$, ranging from $15 \%$ to $50 \%$ [18]. Nevertheless, recent improvements in unquenched lattice QCD yield preliminary calculations of $\left|f_{+}\left(q^{2}\right)\right|$ with much lower uncertainties [24]. Although limited to $B^{0} \rightarrow \pi^{-} \ell^{+} \nu_{\ell}$ at large $q^{2}$, these calculations may open the way to a new era for $\left|V_{u b}\right|$ extraction.

\subsection{Inclusive measurement}

\subsubsection{Duality and the spectator model}

In an inclusive approach, all possible final hadronic states $X_{u}$ are considered, ignoring the detailed breakdown among the individual decay modes that contribute to the semileptonic rate. This offers an advantage over the exclusive approach: the energy released into the state by the decay of the heavy $b$-quark is large such that the final hadronic state is not dominated by a few sharp resonances. If resonances are indeed unimportant, then there is a factorization between the short distance part of the decay (the disappearance of the $b$ quark) and the long distance part (the eventual hadronization of the decay products). This factorization implies that for sufficiently inclusive quantities it is enough to consider 
the short distance part of the process, with the subsequent hadronization taking place with unit probability. This factorization is known as local parton-hadron duality.

The inclusive charmless semileptonic branching fraction may be defined as

$$
\begin{aligned}
\mathcal{B}_{u}^{\mathrm{SL}}=\frac{\Gamma\left(B \rightarrow X_{u} \ell \bar{\nu}_{\ell}\right)}{\Gamma(B \rightarrow \text { anything })} & =\Gamma\left(B \rightarrow X_{u} \ell \bar{\nu}_{\ell}\right) \tau_{B} \\
& =\Gamma_{u}^{\mathrm{SL}} \tau_{B}
\end{aligned}
$$

such that an expression similar to equation 2.19 may be obtained for $\left|V_{u b}\right|$, but without form factors. By ignoring the interactions between the $b$ quark and the light quark, the Spectator Model predicts the decay width of all hadrons containing a $b$ quark and is the simplest way to evaluate $\Gamma_{u}^{\mathrm{SL}}$. Returning to the $b \rightarrow u \ell \bar{\nu}_{\ell}$ process outlined in equation 2.1 and the associated Lagrangian defined in equation 2.3, the matrix element factorises in a leptonic and hadronic current

$$
\mathcal{M}\left(b \rightarrow u \ell \bar{\nu}_{\ell}\right)=i \frac{G_{F}}{\sqrt{2}} V_{u b}\left[\bar{\psi}_{u} \gamma^{\mu}\left(1-\gamma^{5}\right) \psi_{b}\right]\left[\bar{\psi}_{\ell} \gamma_{\mu}\left(1-\gamma^{5}\right) \psi_{\nu}\right]
$$

where $\psi$ denotes the individual Dirac spinors. Assuming the probability for subsequent hadronization of the final $X_{u}$ state to be $100 \%$ (duality), the $B \rightarrow X_{u} \ell \bar{\nu}_{\ell}$ decay rate can then be obtain by integrating $|\mathcal{M}|^{2}$ as

$$
\begin{aligned}
\Gamma_{u}^{\mathrm{SL}} & =\Gamma\left(b \rightarrow u \ell \bar{\nu}_{\ell}\right) \\
& =\frac{G_{F}^{2} m_{b}^{5}}{192 \pi^{3}}\left|V_{u b}\right|^{2} z_{0}\left(m_{u} / m_{b}\right)
\end{aligned}
$$

where $z_{0}(x)=1-8 x^{2}+8 x^{6}-x^{8}-24 x^{4} \ln x$ [22]. Unfortunately, large uncertainties in the quark masses as well as the interactions between the $b$ quark and the light degrees of freedom in the meson have non-negligible effects [25]. These may be accounted for by an expansion of the QCD Lagrangian as will be discussed below.

\subsubsection{Heavy Quark Expansion}

Figure 2.4 provides a more realistic view of a $B \rightarrow X_{u} \ell \bar{\nu}_{\ell}$ semileptonic decay. As such, the $B$ meson can be considered as composed of one heavy $b$ quark, a light antiquark, and a 


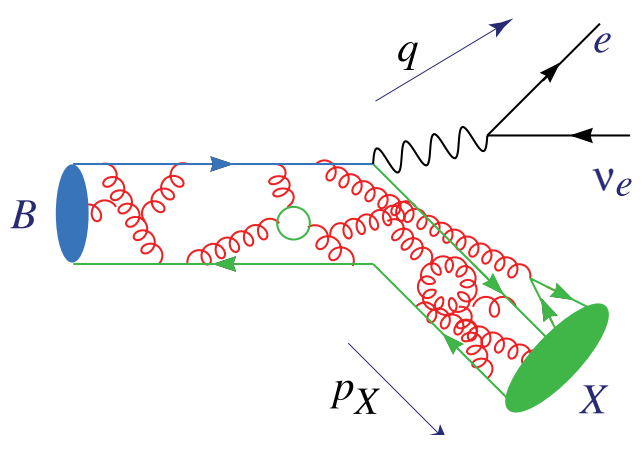

Figure 2.4: Higher order QCD interactions in a semileptonic $B$ decay. Also represented are the kinematic variables used to describe $B \rightarrow X e \bar{\nu}_{e}$ decays.

gluon cloud containing light quark-antiquark pairs. This cloud of virtualities acts such that it keeps all of the meson components together in a colourless bound state. One can then visualize the light cloud as a soft medium in which the heavy $b$ quark is submerged. Ignoring hard gluon exchanges, the momentum which may be transferred from the virtualities to the heavy antiquark is then of order $\Lambda_{\mathrm{QCD}}$. Hence, the $b$ quark moves more or less with the hadron, and the effects of soft gluon exchange may be evaluated in perturbation theory [26]. Historically, Bjorken was the first to discuss the effects due to the heavy quark motion inside heavy hadrons in the problem of heavy quark fragmentation [27]. In application to the heavy hadron decays similar ideas were laid in the basis of the ACCMM model designed as a simple non-relativistic model of the heavy quark motion, which was referred to as the Fermi Motion [28]. These ideas have been incorporated in the Heavy Quark Expansion description of semileptonic $B$ decays.

Effective field theories are based on the principle that in a given process, only certain degrees of freedom may be important for understanding the underlying physics. In particular, kinematical constraints which limit the momenta of external particles effectively restrict the momenta of virtual particles as well. As such, intermediate states of high virtuality may be removed from the theory and replaced by effective interactions among the remaining degrees of freedom. It is then possible to exploit systematically a small expansion 
parameter to obtain a prediction for a given process.

The Heavy Quark Expansion (HQE) is an effective field theory derived from the limit $\Lambda_{\mathrm{QCD}} / m_{b} \rightarrow 0$, and the physics is characterized by virtualities at energy scales $\Lambda_{\mathrm{QCD}}<$ $\mu \leq m_{b}$. The scale, $\mu$, is such that $\alpha_{s}\left(m_{b}\right) / \pi \approx 0.1 \ll 1$, and $\Lambda_{\mathrm{QCD}} / m_{b}<\mu / m_{b} \sim 0.1 \ll 1$. Hence, one may exploit the presence of such small parameters in QCD perturbation theory. Since the $b$ quark inside the $B$ meson is real, it cannot be integrated out. Instead, the $b$ quark interacts with the light degrees of freedom of typical energy $\sim \Lambda_{\mathrm{QCD}}$, which drives the $b$-quark mass off shell by virtualities only of order $\Lambda_{\mathrm{QCD}}$. What can then be integrated out is not the heavy quark itself, but rather those parts of the field which take it far off shell.

In HQE, the Lagrangian is expanded in terms of local operators, the expectation value of which are properties of the $b$ quark within the $B$ meson. Processes with virtual hard gluons which drive the heavy quark far off shell lead to calculable perturbative corrections in the effective theory of order $\alpha_{s}\left(m_{b}\right)$. Further power corrections are necessary and lead to terms of order $\left(\Lambda_{\mathrm{QCD}} / m_{b}\right)^{n}$. These non-perturbative corrections are not calculable from first principle and require the introduction of new phenomenological parameters whose values must be determined experimentally.

\subsubsection{Application of $\mathrm{HQE}$}

Using the Optical Theorem, the decay width of a $B$ meson can be written in the form

$$
\Gamma(B \rightarrow X)=\frac{1}{2 m_{B}} 2 \operatorname{Im}\langle B|\vec{T}| B\rangle,
$$

where the transition operator $\vec{T}$ is given by the time-ordered product of two effective Lagrangians:

$$
\vec{T}=i \int \mathrm{d}^{4} x T\left\{\mathcal{L}_{\text {eff }}(x), \mathcal{L}_{\text {eff }}(0)\right\}
$$



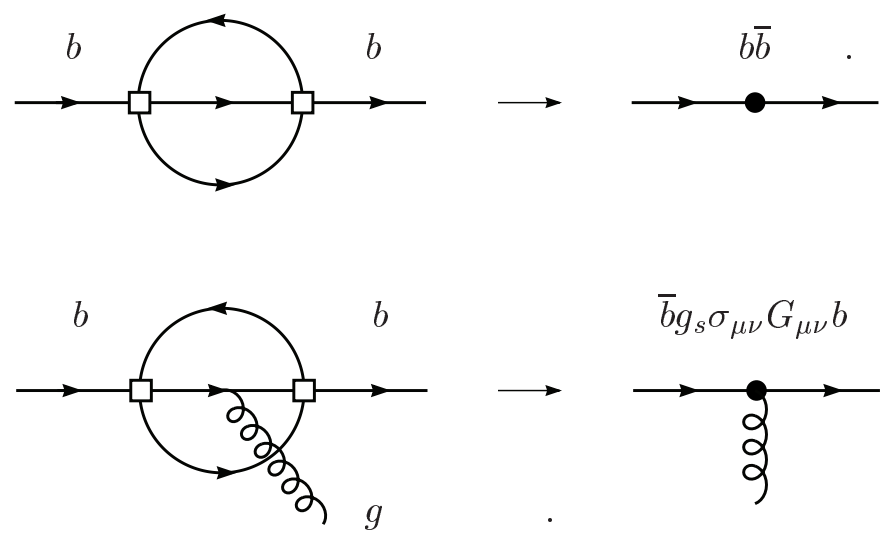

Figure 2.5: Perturbative contributions to the transition operator (left) and the corresponding operators in HQE [31].

and the effective weak Lagrangian $\mathcal{L}_{\text {eff }}$ is given in equation $2.5[29,30] .{ }^{5}$ Hence, if all possible semileptonic states $X$ are considered, we obtain the inclusive charmless decay rate

$$
\Gamma_{u}^{\mathrm{SL}}=\frac{1}{2 m_{B}} \sum_{X}(2 \pi)^{2} \delta^{4}\left(p_{B}-p_{X}\right)\left|\left\langle X\left|\mathcal{L}_{\mathrm{eff}}\right| B\right\rangle\right|^{2} .
$$

Some contributions to the transition operator are given by the two-loop diagrams shown on figure 2.5. It is then possible to construct an expansion of the transition operator, in which $\vec{T}$ is represented as a series of local operators containing the heavy-quark fields. The operator with lowest dimension is $b \bar{b}(d=3)$. It arises from integrating over the internal lines in the first diagram shown in the figure. The equations of motion imply that the matrix elements of dimension-four operators vanish. Thus, the leading non-perturbative corrections arise from $d=5$ operators which contain the gluon field-strength tensor and are of order $\left(1 / m_{b}^{2}\right)$. It originates from diagrams in which a gluon is emitted from one of the internal lines, such as the second diagram shown in figure 2.5.

At higher order, a large number of operators appear, but for dimensional reasons, the matrix elements of these operators are suppressed by inverse powers of the $b$-quark

\footnotetext{
${ }^{5}$ A comprehensive overview of the following calculations may be found in [31].
} 
mass. Therefore, the inclusive charmless semileptonic rate may be written as $[29,30]$

$$
\Gamma_{u}^{\mathrm{SL}}=\frac{G_{F}^{2} m_{b}^{5}}{192 \pi^{3}}\left|V_{u b}\right|^{2}\left\{c_{3}^{f}\langle\bar{b} b\rangle_{B}+c_{5}^{f} \frac{\left\langle\bar{b} g_{s} \sigma_{\mu \nu} G_{\mu \nu} b\right\rangle_{B}}{m_{b}^{2}}+\cdots\right\},
$$

where $c_{n}^{f}$ are calculable short-distance coefficient functions depending on the quantum numbers of the final states $f$, and $\langle O\rangle_{B}$ are the normalized forward matrix elements of local operators, written using the short-hand notation

$$
\langle O\rangle_{B}=\frac{1}{2 m_{B}}\langle B|O| B\rangle .
$$

These matrix elements, which contain all of the long-distance contributions, can be systematically expanded in powers of $1 / m_{b}$ and yield

$$
\langle\bar{b} b\rangle=1+\frac{\lambda_{1}+3 \lambda_{2}}{2 m_{b}^{2}}+\mathcal{O}\left(1 / m_{b}^{3}\right)
$$

and

$$
\left\langle\bar{b} g_{s} \sigma_{\mu \nu} G_{\mu \nu} b\right\rangle=6 \lambda_{2}+\mathcal{O}\left(1 / m_{b}^{3}\right)
$$

The quantity

$$
\lambda_{1}=\frac{1}{2 m_{B}}\left\langle B\left|\bar{b}(i \vec{D})^{2} b\right| B\right\rangle
$$

is the average momentum squared of the $b$ quark inside the $B$ meson, whereas

$$
3 \lambda_{2}=\frac{1}{2 m_{B}}\left\langle B\left|\bar{b} \frac{g_{s}}{2} \sigma_{\mu \nu} G^{\mu \nu} b\right| B\right\rangle
$$

is the Lorentz invariant chromomagnetic interaction energy [29]. Note that the expectation value of the kinetic operator is introduced in the literature also as $\mu_{\pi}^{2}=-\lambda_{1}$, and the expectation value of the chromomagnetic operator as $\mu_{G}^{2}=3 \lambda_{2}$ [30]. The latter may be obtained experimentally from the mass splitting between $B$ and $B^{*}$ as $m_{B^{*}}^{2}-m_{B}^{2}=$ $4 \lambda_{2} \simeq 0.48 \mathrm{GeV}^{2}[18]$. On the other hand, $\lambda_{1}$ cannot be obtained from hadron spectroscopy. Instead, one may use the photon energy spectrum from $B \rightarrow X_{s} \gamma$ decays or the lepton energy and hadronic mass moments from $B \rightarrow X_{c} \ell \bar{\nu}_{\ell}$ decays as will be discussed in section 4.2.2. Combining equations 2.29 and 2.32 yields

$$
\Gamma_{u}^{\mathrm{SL}}=\frac{G_{F}^{2} m_{b}^{5}}{192 \pi^{3}}\left|V_{u b}\right|^{2}\left\{c_{3}^{f}\left(1+\frac{\lambda_{1}+3 \lambda_{2}}{2 m_{b}^{2}}\right)+c_{5}^{f} \frac{6 \lambda_{2}}{m_{b}^{2}}+\mathcal{O}\left(1 / m_{b}^{3}\right)\right\} .
$$




\subsubsection{Weak annihilation}

The size of the contribution from four-quark operators of dimension six to the total $B \rightarrow$ $X_{u} \ell \bar{\nu}_{\ell}$ decay rate presented in the previous section has been shown to be non-negligible [32]. This contribution, or weak annihilation, corresponds to a small portion of the momentum of order $\mathcal{O}\left(1 / m_{b}^{3}\right)$ carried away by the final light quark, and all the momentum of the initial heavy quark flowing through the lepton pair. Thus, this contribution appears only around $q^{2} \simeq m_{b}^{2}$, and constitutes a fixed fraction of the total rate of the $B \rightarrow X_{u} \ell \bar{\nu}_{\ell}$ decays, about $\sim 3 \%$ [33]. However, this effect may be enhanced since kinematic constraints must be applied to remove charm background and typically result in selecting a portion of the phase-space with higher $q^{2}$-value.

\subsubsection{Perturbative corrections and $b$-quark mass}

As mentioned in section 2.5.3, the coefficients $c_{n}^{f}$ appearing in equation 2.32 are purely perturbative corrections. They are computed in a $\alpha_{s}(\mu) / \pi$ expansion of the form

$$
\Gamma_{u}^{\mathrm{SL}}=\frac{G_{F}^{2} m_{b}(\mu)^{5}}{192 \pi^{3}}\left|V_{u b}\right|^{2}\left[1+a_{1} \frac{\alpha_{s}(\mu)}{\pi}+a_{2}\left(\frac{\alpha_{s}(\mu)}{\pi}\right)^{2}+\cdots\right] .
$$

The important feature of the above, is that the $b$-quark mass as well as the strong coupling are running: they depend on the normalization point $\mu$. At the scale $\mu=m_{b}$,

$$
\Gamma_{u}^{\mathrm{SL}}=\frac{G_{F}^{2} m_{b}^{5}}{192 \pi^{3}}\left|V_{u b}\right|\left[1-2.41 \frac{\alpha_{s}}{\pi} \epsilon-3.22 \frac{\alpha_{s}^{2}}{\pi^{2}} \beta_{0} \epsilon^{2}+\frac{\lambda_{1}-9 \lambda_{2}}{2 m_{b}^{2}}+\cdots\right],
$$

where $\beta_{0}=11-2 n_{f} / 3$ is the one-loop beta function of QCD, and $\epsilon \equiv 1$ denotes the order of the expansion [34].

Unfortunately, there is an ambuiguity of order $\sim \Lambda_{\mathrm{QCD}}$ in defining the $b$-quark mass. In fact, the main uncertainty in theoretical predictions of $\Gamma_{u}^{\mathrm{SL}}$ arise from the poorly known $b$-quark mass which defines the phase space, and cause a $20 \%$ uncertainty in the perturbative corrections when it is written in terms of the pole mass [35]. Instead, various techniques are used in order to obtain an expressions for extracting $\left|V_{u b}\right|$ from a measurement of the charmless semileptonic branching fraction, but with reduced sensitivity to renormalization 


\begin{tabular}{|l|c|c|}
\hline Authors & Decay type & $A\left(\times 10^{-3}\right)$ \\
\hline \hline Bigi, Shifman, Uraltsev [26] & $B \rightarrow X_{u} e \overline{\nu_{e}}$ & $4.48 \pm 0.13$ \\
Hoang, Ligeti, Manohar [34] & $B \rightarrow X_{u} e \overline{\nu_{e}}$ & $4.29 \pm 0.10$ \\
Bigi [36] & $B \rightarrow X_{u} \ell \overline{\nu_{\ell}}$ & $8.95 \pm 0.25$ \\
Becher, Neubert [37] & $B \rightarrow X_{u} \ell \overline{\overline{\nu_{\ell}}}$ & $9.76 \pm 0.70$ \\
\hline
\end{tabular}

Table 2.4: Various theoretical results for $\left|V_{u b}\right|$ in terms of the semileptonic branching fraction: $\left|V_{u b}\right|=A \times\left(\frac{\mathcal{B}\left(B \rightarrow X_{u} \ell \overline{\nu_{\ell}}\right)}{0.002} \frac{1.61 \mathrm{ps}}{\tau_{B}}\right)^{1 / 2}$, where $\ell=e$ and $\mu$. Note that the dominant theoretical error comes from the computation of $\mathcal{B}_{u}^{\mathrm{SL}}$, and is not included.

of $m_{b}$. Some of these results are shown in table 2.4. All of these predictions are in good agreement and yield to an average of

$$
\left|V_{u b}\right|=0.000424 \sqrt{\frac{\mathcal{B}\left(B \rightarrow X_{u} e \bar{\nu}_{e}\right)}{0.0002} \frac{1.61 \mathrm{ps}}{\tau_{B}}}(1 \pm 0.048),
$$

after updating the HQE parameters to the latest values [18]. Hence, $\left|V_{u b}\right|$ may be determined with a $\sim 5 \%$ precision using a measurement of the inclusive $B \rightarrow X_{u} \ell \bar{\nu}_{\ell}$ branching ratio.

\subsubsection{Shape Function}

Since the ratio $\left|V_{c b}\right| /\left|V_{u b}\right| \sim 10$, the $B \rightarrow X_{c} \ell \bar{\nu}_{\ell}$ rate is predicted to dominate over that of the $B \rightarrow X_{u} \ell \bar{\nu}_{\ell}$ by a ratio of about 100:1. Nonetheless, these background decays may be discriminated from the signal $B \rightarrow X_{u} \ell \bar{\nu}_{\ell}$ by looking at the phase-space boundaries for $B \rightarrow X_{c} \ell \bar{\nu}_{\ell}$ transitions. In principle, three observables can be used to determine such boundaries:

- The hadronic mass: $m_{X}=m_{D^{0}}=1.865 \mathrm{GeV} / c^{2}$;

- The charged lepton energy: $E_{\ell}=\left(m_{B}^{2}-m_{D^{0}}^{2}\right) c^{2} / 2 m_{B} \simeq 2.3 \mathrm{GeV}$;

- The dilepton invariant mass squared: $q^{2}=\left(m_{B}-m_{D}\right)^{2} \simeq 11.7 \mathrm{GeV}^{2} / c^{4}$.

Of course, one could also use a combination of the above. Any measurement of $\left|V_{u b}\right|$ will therefore be restricted to a small portion of the phase space in order to suppress the dominant charm background. A theoretical model must then be used in order to extrapolate 
the partial $B \rightarrow X_{u} \ell \bar{\nu}_{\ell}$ branching fraction measurement to the full phase space, which implies further theoretical uncertainties.

In an effort to provide such a model, De Fazio and Neubert [38] evaluated the triple differential decay width $d \Gamma_{u}^{\mathrm{SL}} / d x d z d p^{2}$; these three variables have the following meaning at the parton level

$$
x=\frac{2 E_{\ell}}{m_{b}}, z=\frac{2\left(E_{X_{u}}-\bar{\Lambda}\right)}{m_{b}}, \text { and } p^{2}=\frac{m_{X_{u}}^{2}-2 E_{X_{u}} \bar{\Lambda}-\bar{\Lambda}^{2}}{m_{b}^{2}},
$$

where $E_{X_{u}}$ and $m_{X_{u}}$ are the energy and mass of the hadronic state $X_{u}$, and $\bar{\Lambda}=m_{B}-m_{b}$ is the binding energy. The effects of the Fermi motion on the partial decay width are important when studying the prediction for inclusive decay on a scale smaller than $m_{b}$, especially in the low mass region $m_{X}=\mathcal{O}(\Lambda)$. In this approach, these effects are parameterized using a Shape Function $(\mathrm{SF})$ which defines the momentum distribution of the heavy quark inside the $B$ meson. A model $\mathrm{SF}$ is given by the exponential form

$$
F\left(k_{+}\right)=N(1-x)^{a} e^{(1+a) x} ; \quad x=\frac{k_{+}}{\bar{\Lambda}} \leq 1
$$

where the $b$-quark mass, explicitely defined in the context of the SF, $m_{b}=m_{B}-\bar{\Lambda}$, and the dimensionless parameter $a$ are the two free parameters. Other ansätze have been suggested in the literature to describe the SF such as the Gaussian and Roman shape functions [30, 39]. The Gaussian SF is parameterized as

$$
F\left(k_{+}\right)=N(1-x)^{a} e^{-b^{2}(1+x)^{2}} ; \quad b=\Gamma\left(\frac{a+2}{2}\right) / \Gamma\left(\frac{a+1}{2}\right),
$$

where $x$ is defined as per equation 2.37, whereas the Roman SF is defined as

$$
F\left(k_{+}\right)=N \frac{\kappa}{\pi} e^{-\frac{1}{4}\left(\frac{1}{\kappa} \frac{a}{1-x}-\kappa(1-x)\right)^{2}} ; \quad \kappa=\frac{a}{\pi} e^{a / 2} K_{1}(a / 2),
$$

where $K_{1}$ refers to the modified Bessel equation of the first kind. These ansätze, including the exponential form, must satisfy conditions imposed on the first three $k_{+}$moments,

$$
A_{n}=\int_{-\infty}^{\bar{\Lambda}} d k_{+} k_{+}^{n} F\left(k_{+}\right)
$$



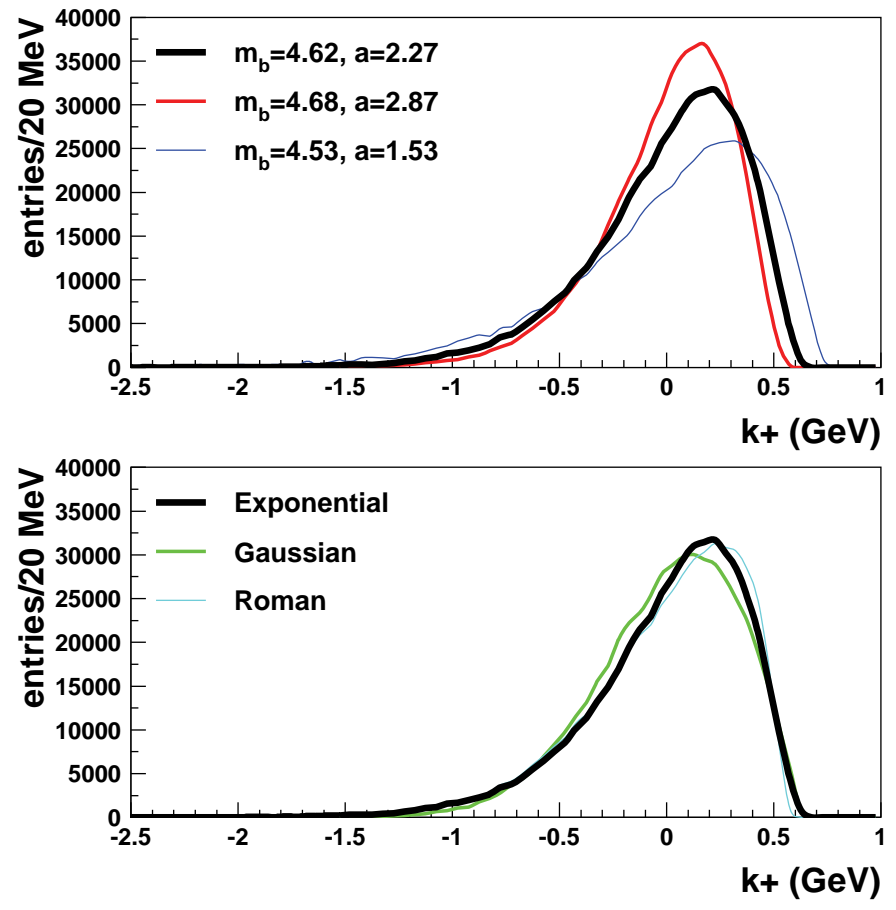

Figure 2.6: Fermi motion spectra obtained using various heavy quark parameters (top) and ansätze (bottom). The nominal choice is displayed in black. The choice of HQE parameters correspond to results presented in [40] and [41].

namely: $A_{0}=1, A_{1}=0$, and $A_{2}=-\lambda_{1} / 3$. Figure 2.6 displays the momentum spectra expected for various combinations of the HQE parameters or ansätze. In all cases, $k_{+}$can take values between $-m_{b}$ and $\bar{\Lambda}$, with a distribution centered around $k_{+}=0$. Cleary, one can also see from figure 2.6, that the uncertainties on the mass of the $b$ quark and parameter $a$ have non-negligible effects on $F\left(k_{+}\right)$. The choice of HQE parameters will be discussed in section 4.2.2.

The convolution of parton spectra with the function $F\left(k_{+}\right)$requires that the $b$-quark mass be replaced by the momentum dependent mass $m_{b}+k_{+}$in the expression for the shape function, i.e.

$$
d \Gamma=\int d k_{+} F\left(k_{+}\right) d \Gamma_{\text {parton }}\left(m_{b}+k_{+}\right)
$$


a)

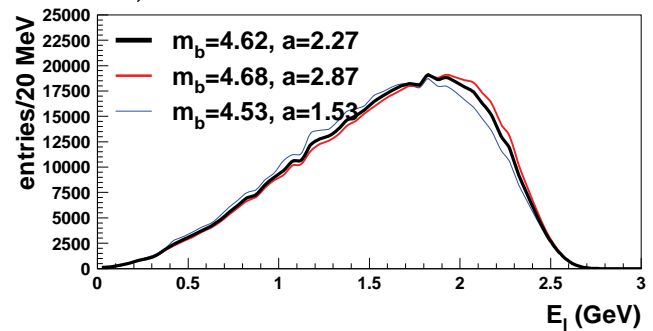

c)

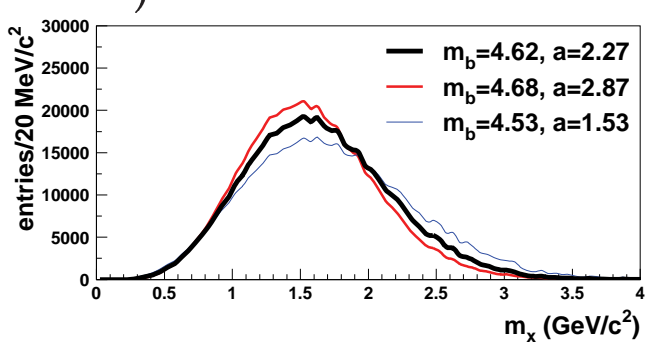

b)
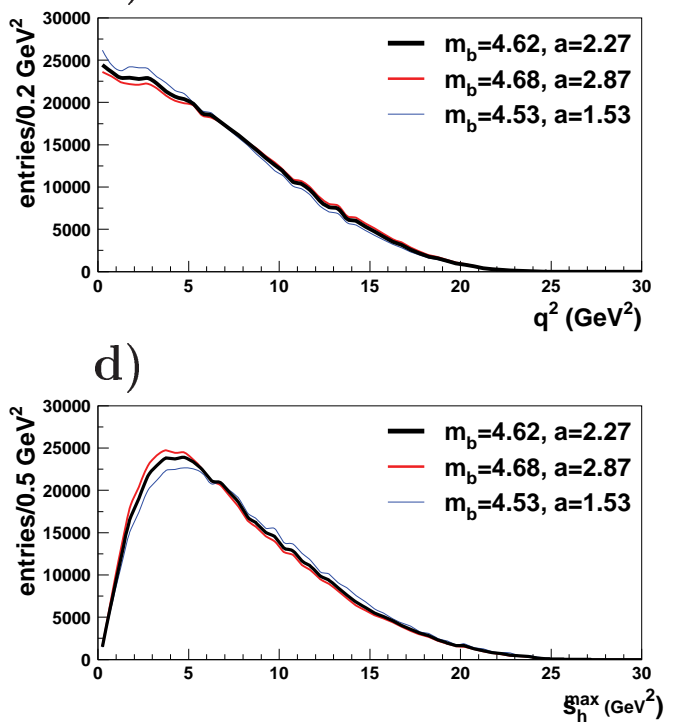

Figure 2.7: Monte Carlo distribution for a) the lepton energy, b) $q^{2}$, c) the hadronic mass and d) $s_{\mathrm{h}}^{\max }$ for $B \rightarrow X_{u} e \bar{\nu}_{e}$ decays using various heavy quark parameters. The convolution of the parton spectra with the shape function effectively smears out the various spectra. The hadronic mass is most affected by the SF uncertainties, whereas $q^{2}$ is the least affected quantity. The quantity $s_{\mathrm{h}}^{\max }$ is defined in section 2.7 .

The lepton energy, hadronic mass and $q^{2}$ spectra are effectively smeared out by the Fermi motion $[29,30]$, and the error on this smearing is a function of the difference between the various distribution shown on figure 2.7. In order to make an improved measurement of $\left|V_{u b}\right|$, it is then crucial to use an observable (or set of observables) which both maximizes the fraction of the $B \rightarrow X_{u} \ell \bar{\nu}_{\ell}$ phase-space probed and minimizes the sensitivity to the theoretical uncertainties. The choice of variable(s) is then a compromise between these two ideals to minimize the overall uncertainty on $\left|V_{u b}\right|$. 


\subsection{Previous extractions of $\left|V_{u b}\right|$}

Prior to this analysis, two observables have been used to determine $\left|V_{u b}\right|$ from inclusive semileptonic $B$ decays: the endpoint of the lepton momentum spectrum [6] and the mass of the accompanying hadronic system [7]. Until recently, endpoint studies were limited to a small slice of the lepton momentum spectrum between 2.3 to $2.6 \mathrm{GeV} / \mathrm{c}$. This represents only $\sim 10 \%$ of the $B \rightarrow X_{u} \ell \bar{\nu}_{\ell}$ rate, the remaining $90 \%$ being hidden underneath the dominant $B \rightarrow X_{c} \ell \bar{\nu}_{\ell}$ transition. Hence, large extrapolations were required in order to obtain the full $B \rightarrow X_{u} \ell \bar{\nu}_{\ell}$ rate and a significant amount of background still remained. Furthermore, there was some skepticism regarding theoretical uncertainties as non-perturbative QCD corrections were thought to affect the value of $\left|V_{u b}\right|$ by as much as $10 \%$ [42].

Measurements based on the reconstruction of the invariant mass $m_{X}$ of the hadronic system in semileptonic $B$ decays and on simultaneous requirements on $m_{X}$ and $q^{2}$ had also been performed [7]. In order to determine $m_{X}$ with good resolution, a large sample of fully reconstructed $B$ mesons were used, thereby allowing the proper assignment of particles to the hadronic and semileptonic $B$ mesons in the event. This required large statistics, since the efficiency to fully reconstruct the hadronic $B$ decay is a few per mille. Requirements on the hadronic mass of $m_{X}<1.55 \mathrm{GeV} / c^{2}$ were made to select a clean sample of $B \rightarrow X_{u} \ell \bar{\nu}_{\ell}$ decays. As mentioned in [33], further requirements on $q^{2}$ were also imposed in order to reduce theoretical uncertainties. Based on the above, a measurement was made using a "simulated annealing" to determine which particles to associate with the semileptonic $B$. This resulted in a rather poor resolution on both $m_{x}$ and $q^{2}$.

Results based on the above methods are presented in figure 2.8. These were obtained prior to the work shown in this dissertation. Hence, the goal of this study is to improve the precision on $\left|V_{u b}\right|$ with respect to these determinations. New measurements have become available while this work was nearing completion and are compared to the results obtained in this work in chapter 8. 


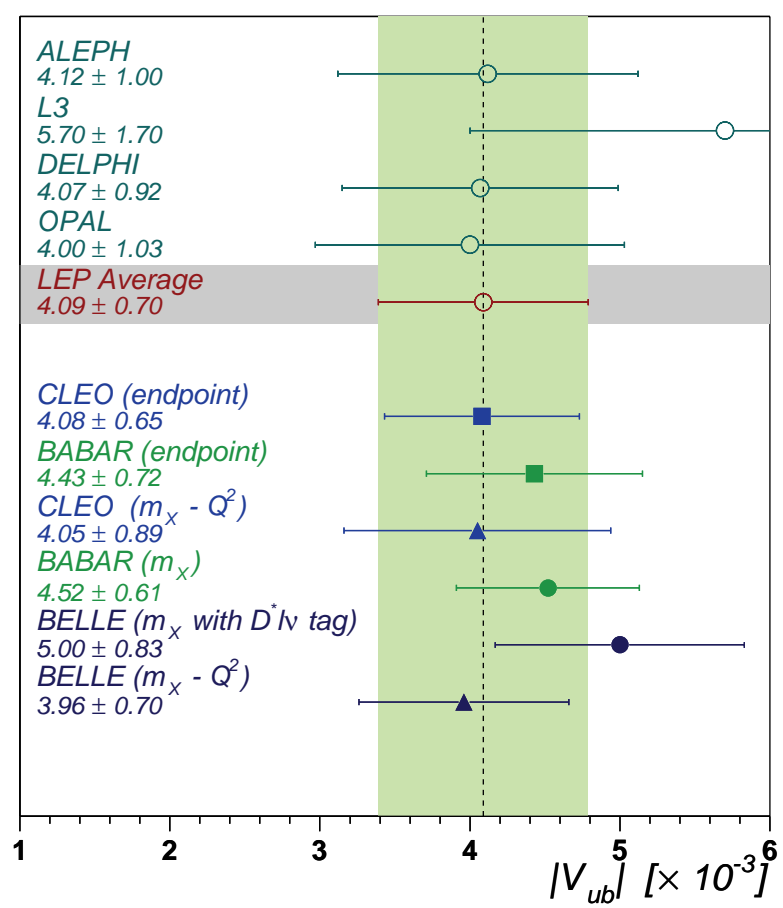

Figure 2.8: Status of $\left|V_{u b}\right|$ measurements prior to this work (winter 2002 [19]). An additional $5 \%$ error must be added to the above results to account for the determination of $\left|V_{u b}\right|$ from $\mathcal{B}_{u}^{\mathrm{SL}}$ (equation 2.35).

\subsection{The $q^{2}-E_{\ell}^{*}$ approach}

The expected distribution for of $q^{2}$ vs $E_{e}^{* 6}$ for $B \rightarrow X_{u} \ell \bar{\nu}_{\ell}$ decays has been obtained through generator-level Monte Carlo simulations and is displayed in figure 2.9. Also shown in figure 2.9 is the expected background from $B \rightarrow X_{c} \ell \bar{\nu}_{\ell}$, where the beauty quark decays to charm. As mentioned above, background events dominate over $B \rightarrow X_{u} \ell \bar{\nu}_{\ell}$ events by a ratio $\sim 100: 1$. Nonetheless, these events may be discriminated from the signal events by looking at the phase-space boundaries of $B \rightarrow X_{c} \ell \bar{\nu}_{\ell}$ transitions in the $q^{2}-E_{\ell}^{*}$ plane. Semileptonic decays are selected based on the presence of a high energy lepton, and the determination of the $\ell \bar{\nu}_{\ell}$ invariant mass squared, $q^{2}$, by combining the measured charged lepton momen-

\footnotetext{
${ }^{6} E_{\ell}^{*}$ is the charged lepton energy in the center-of-mass frame.
} 

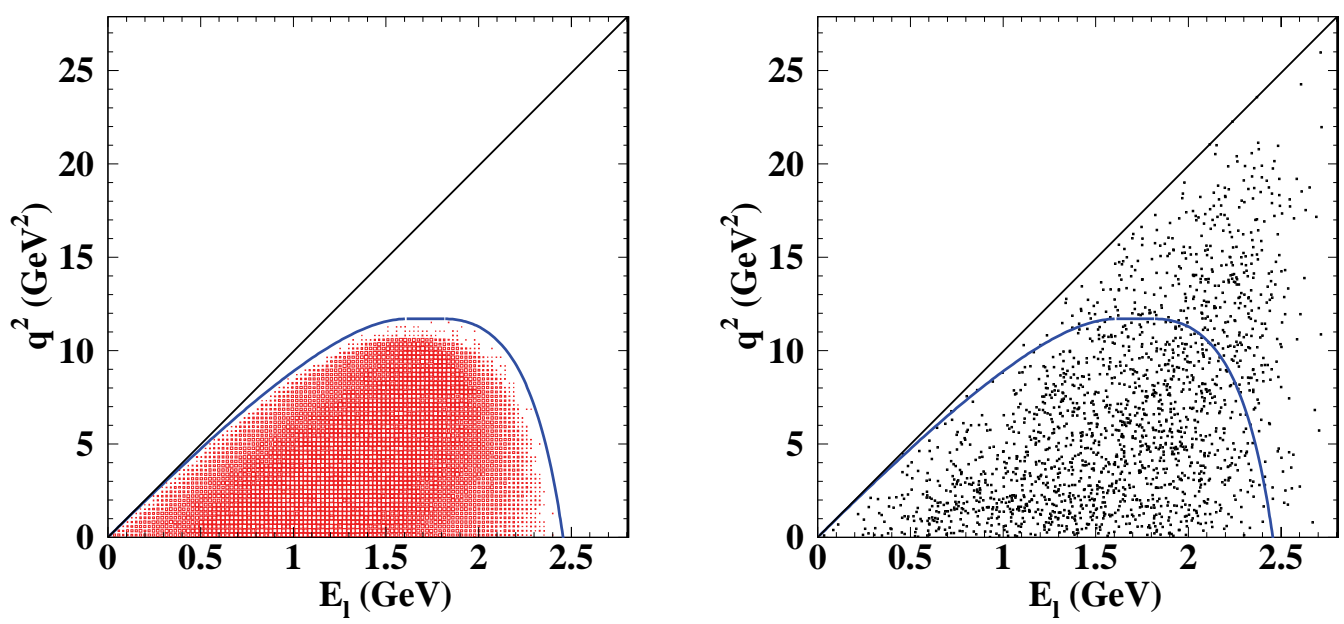

Figure 2.9: Distribution of $B \rightarrow X_{c} \ell \bar{\nu}_{\ell}$ (left) and $B \rightarrow X_{u} \ell \bar{\nu}_{\ell}$ (right) decays in the $q^{2}-E_{\ell}^{*}$ plane. The energy of the charged lepton, $E_{\ell}^{*}$, is given in the center of mass frame, and $q^{2}$ is the invariant of the lepton-neutrino system.

tum with an estimate of the neutrino momentum based on a measurement of the missing momentum of the event. $E_{\ell}^{*}$ and $q^{2}$ are combined to compute the maximum kinematically allowed invariant mass squared of the hadronic recoil system, $s_{\mathrm{h}}^{\max }[8] .{ }^{7}$ In the case where $\pm E_{\ell}^{*}> \pm \frac{\sqrt{q^{2}}}{2}\left(\frac{1 \pm \beta}{1 \mp \beta}\right),{ }^{8}$ the maximum invariant hadronic mass squared is

$$
s_{\mathrm{h}}^{\max }=m_{B}^{2}+q^{2}-2 m_{B} E_{\ell}^{*} \sqrt{\frac{1 \mp \beta}{1 \pm \beta}}-2 m_{B}\left(\frac{q^{2}}{4 E_{\ell}}\right) \sqrt{\frac{1 \pm \beta}{1 \mp \beta}},
$$

otherwise,

$$
s_{\mathrm{h}}^{\max }=m_{B}^{2}+q^{2}-2 m_{B} \sqrt{q^{2}} .
$$

The above variables are all expressed in the $\Upsilon(4 \mathrm{~S})$ rest frame. Constant values of $s_{\mathrm{h}}^{\max }$ define the contour lines in the $q^{2}-E_{\ell}^{*}$ plane as shown in figure 2.9. $B \rightarrow X_{u} \ell \bar{\nu}_{\ell}$ events are contained within the region defined by $s_{\mathrm{h}}^{\max }=0$ (diagonal contour), whereas events containing $B \rightarrow X_{c} \ell \bar{\nu}_{\ell}$ decays can be removed by requiring $s_{\mathrm{h}}^{\max }<m_{D}^{2}$ (blue contour). Hence, no events with a true hadronic recoil mass above $m_{D}$ can enter the sample unless $q^{2}$ or $E_{\ell}^{*}$ is mis-reconstructed.

\footnotetext{
${ }^{7}$ A derivation of $s_{\mathrm{h}}^{\max }$ is provided in appendix A.

${ }^{8}$ Where $\beta=\sqrt{1-\left(2 m_{B} / m_{\Upsilon(4 S)}\right)^{2}} \simeq 0.06$.
} 


\subsection{Comparison with other approaches}

The $q^{2}-E_{\ell}^{*}$ analysis is complementary to analyses based on the lepton momentum endpoint and $m_{X}$ reconstruction. The selection efficiency is higher for the $q^{2}-E_{\ell}^{*}$ analysis than for $m_{x}$ studies, while the signal to background ratio is better than for the endpoint method but worse than studies based on $m_{x}$. Although the $q^{2}-E_{\ell}^{*}$ analysis accepts a smaller fraction of the $B \rightarrow X_{u} \ell \bar{\nu}_{\ell}$ phase space than the $m_{X}$ analyses, it is much less sensitive to shape function uncertainties. This is shown in figure 2.7, where the region corresponding to accepted $B \rightarrow X_{u} \ell \bar{\nu}_{\ell}$ decays is below $1.55 \mathrm{GeV} / c^{2}$ for the $m_{X}$ spectrum, and below $3.5 \mathrm{GeV}^{2}$ for $s_{\mathrm{h}}^{\max }$. Hence, the uncertainty due to Fermi motion when extrapolating the fraction of $B$ decaying to $X_{u} \ell \nu$ over the full spectrum will be smaller using $s_{\mathrm{h}}^{\max }$. On the other hand, non-factorizable effects such as subleading SFs and weak annihilation lead to non-negligible uncertainties. The latter has a larger impact on the determination of $\left|V_{u b}\right|$ from the $q^{2}$ $E_{\ell}^{*}$ analysis than for the $m_{X}$ analyses due to the portion of the $B \rightarrow X_{u} \ell \bar{\nu}_{\ell}$ phase-space probed. While a measurement of the separate charmless semileptonic branching fractions for charged and neutral $B$ mesons could address this uncertainty, this cannot be done in this analysis. 


\title{
Chapter 3
}

\section{The BABAR Experiment}

\author{
Sapiens nihil affirmat quod non probet
}

The primary goal of the BABAR experiment is the systematic study of $C P$ asymmetries in the decays of neutral $B$ mesons. The BABAR experiment also studies a range of other $B$ physics such as the search for rare $B$ meson decays and the measurement of the CKM element $\left|V_{u b}\right|$. The physics of the charm quark and tau leptons as well as hadron spectroscopy are also investigated at BABAR.

Several studies were conducted in the late 1980s and it was determined that an asymmetric $e^{+} e^{-}$collider operating at $\sqrt{s}=10.58 \mathrm{GeV}$, the $\Upsilon(4 S)$ resonance, would be best to study $C P$ violation in $B$ mesons. Table 3.1 shows the various production cross-section for $e^{+} e^{-}$collisions at $\sqrt{s}=10.58 \mathrm{GeV}$. Accordingly, the cross-section for the production of the $b \bar{b}$ state, $e^{+} e^{-} \rightarrow \Upsilon(4 S)$, is $1.05 \mathrm{nb}$, which subsequently decays $50 \%$ of the time to $B^{+} B^{-}$ and $50 \%$ to $B^{0} \overline{B^{0}}$. Furthermore, these $B$ mesons are almost at rest and have no additional particles associated with them, e.g. $B \bar{B} \pi$ is kinematically suppressed. By optimizing the colliding beams with asymmetric energies, it is then possible to distinguish between the decay vertices of the $B$ and $\bar{B}$ due to the longitudinal boost of the centre-of-mass in the lab frame. Without this boost, the relative distance between the two $B$ vertices would be too small ( $\sim 30$ microns) to be measured by any vertex tracker. Finally, it was understood that the machine must have an unprecedented luminosity ${ }^{1}$ in order to provide enough $B$

\footnotetext{
${ }^{1}$ The luminosity is defined as the rate of particles per unit area.
} 


\begin{tabular}{|c|c|}
\hline Decay type & Cross-section (nb) \\
\hline \hline$e^{+} e^{-} \rightarrow b \bar{b}$ & 1.05 \\
$e^{+} e^{-} \rightarrow c \bar{c}$ & 1.30 \\
$e^{+} e^{-} \rightarrow s \bar{s}$ & 0.35 \\
$e^{+} e^{-} \rightarrow u \bar{u}$ & 1.39 \\
$e^{+} e^{-} \rightarrow d \bar{d}$ & 0.35 \\
\hline$e^{+} e^{-} \rightarrow \tau^{+} \tau^{-}$ & 0.89 \\
$e^{+} e^{-} \rightarrow \mu^{+} \mu^{-}$ & 1.16 \\
$e^{+} e^{-} \rightarrow e^{+} e^{-}$ & $\sim 40$ \\
\hline
\end{tabular}

Table 3.1: Cross-section for $e^{+} e^{-}$collisions at $\sqrt{s}=10.58 \mathrm{GeV}$ [43].

mesons. These characteristics were used in the design of the PEP-II collider.

The BABAR detector was optimized to study the millions of $B \bar{B}$ meson pairs produced by the PEP-II collider. Since the center-of-mass is boosted in the electron forward direction, the detector needs to be asymmetric for maximum acceptance. In order to measure the $C P$ asymmetries in the decays of neutral $B$ mesons, the detector needs an excellent vertex resolution to accurately measure the difference in the decay times of the two $B$ mesons $\Delta t$. In fact, the $B$ mesons travel in a direction almost parallel to the beam pipe (z-axis) such that $\Delta t$ is evaluated from the difference between the z-components of their decay positions. The detector must perform a precise measurement of the charged particle trajectory and photon momenta, and provide an excellent identification of electrons, muons and kaons in order to determine the flavour of at least one $B$ meson and to distinguish as many decay channels as possible.

A longitudinal section of the BABAR detector is shown in figure 3.1. It can be decomposed into five major subdetector systems:

- A silicon vertex tracker (SVT) which performs precision measurement on the position information of charged tracks at the heart of $B A B A R$;

- A drift chamber (DCH) which measures momentum and position of charged particles and identifies particles via the measurement of the rate of energy loss $(\mathrm{dE} / \mathrm{dx})$; 


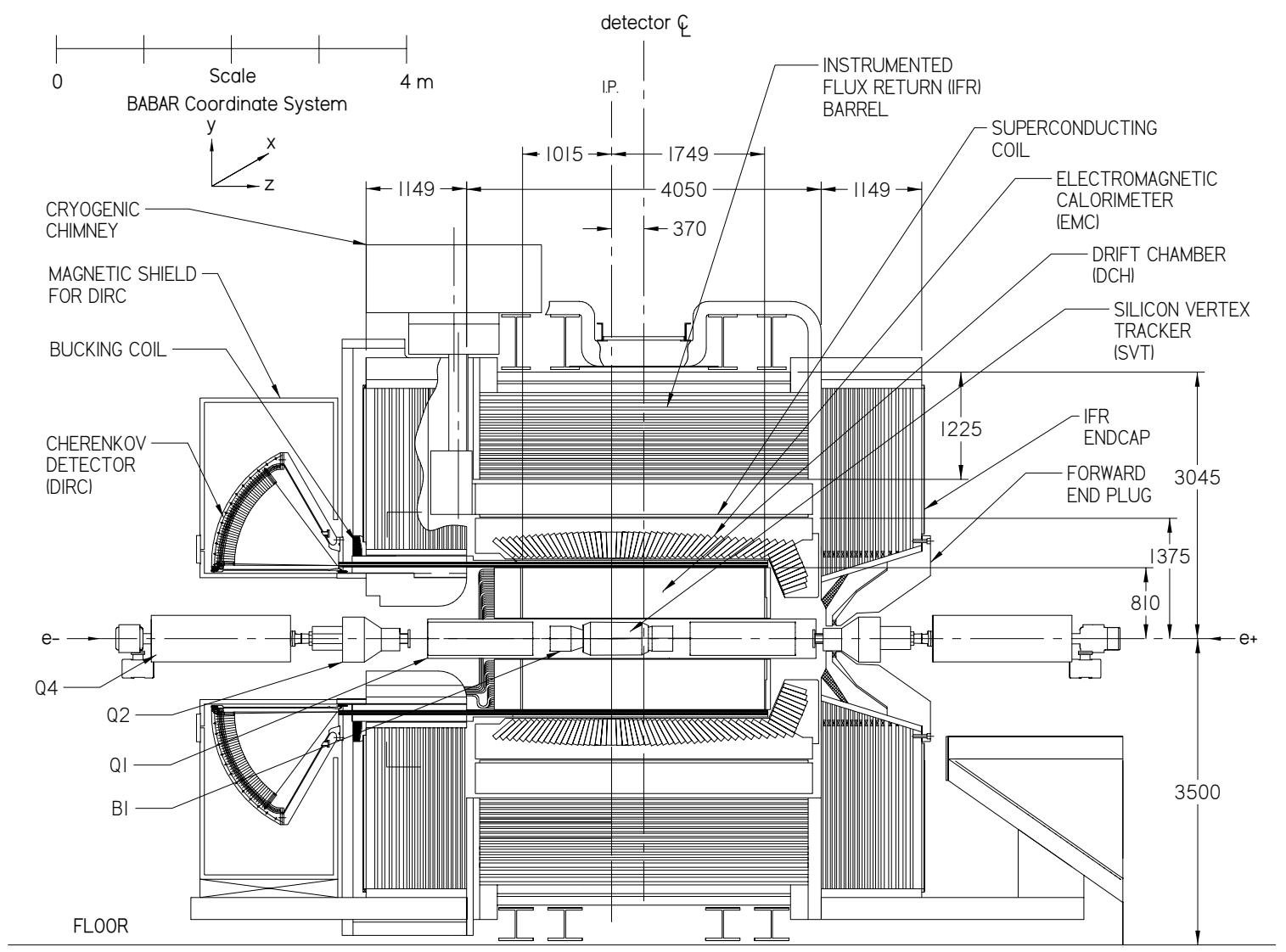

Figure 3.1: Longitudinal view of the BABAR detector. Unless referenced otherwise, all of the figures appearing in this chapter have been obtained from [44].

- A detector of internally reflected Cherenkov light (DIRC) which is used in identifying muons and charged hadrons, especially kaons;

- A Caesium Iodide electromagnetic calorimeter (EMC) which measures the energy and identifies photons and electrons;

- And an instrumented flux return (IFR) used for muon and neutral hadron identification.

There is also a superconducting coil located between the EMC and IFR which provides a 1.5 tesla solenoidal magnetic field needed for the proper operation of the SVT and DCH. 
The detector geometry is cylindrical for the tracking system and hexagonal for the outer structures. It covers most of the $4 \pi$ solid angle: between $350 \mathrm{mrad}$ in the forward region to $400 \mathrm{mrad}$ in the backward direction. Note that the BABAR coordinate system is used throughout this dissertation and is defined in the following way:

- The $z$ axis is parallel to the magnetic field of the solenoid and in the direction of the $e^{-}$beam;

- The $y$ axis points vertically upwards;

- The $x$ axis points horizontally, away from the center of the PEP-II ring.

The origin, $(0,0,0)$, corresponds to the interaction point. The angle $\theta$ is then the angular displacement from the $z$ axis, whereas the angle $\phi$ is defined as the angular displacement from the $x$ axis in the $x-y$ plane.

A trigger system is used to separate collisions producing interesting events from background such as beam interactions with residual gas. The trigger system is divided in two consequent levels: the first level trigger is hardware based and is designed to have a maximum output rate of about $2 \mathrm{kHz}$ and a maximum time delay of $12 \mu \mathrm{s}$, while the other level is software based and has a throughput rate limited to $\sim 200 \mathrm{~Hz}$ in order to permit an easy storage and processing of collected data.

In the following sections, a description of the PEP-II collider is first made and is followed by an overview of the various $B A B A R$ detector components and their performance. A more indepth description of the detector may be found in [44].

\subsection{The PEP-II collider}

Figure 3.2 gives a schematic representation of the linear accelerator and PEP-II collider, located at the Stanford Linear Accelerator Center. In order to achieve $e^{+} e^{-}$collisions at $\sqrt{s}=10.58 \mathrm{GeV}$, high momentum electrons and positrons are first extracted from the linear accelerator and injected into PEP-II which consists of two independent storage rings, 


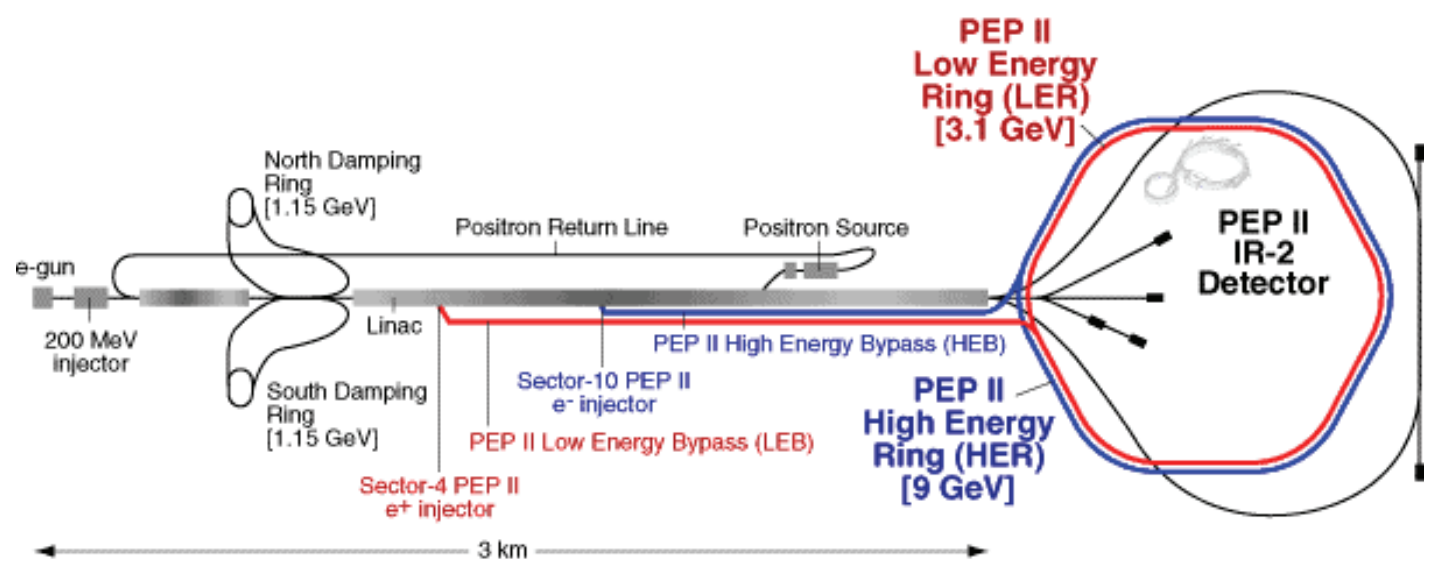

Figure 3.2: Schematic view of the linear accelerator and PEP-II collider at SLAC [44].

one located atop the other. In the high-energy ring (HER), $9 \mathrm{GeV}$ electrons are stored whereas in the low-energy ring (LER), $3.1 \mathrm{GeV}$ positrons are kept traveling in a direction opposite to the electrons. The counter-rotating beams are then collided at the experiment. The asymmetry in the beam energies creates a center-of-mass boost of $\beta \gamma=0.56$ in the direction of the electrons. The $B$ meson lifetime is about $1.6 \mathrm{ps}$, such that the decay vertices of the $B$ mesons are on average about 270 microns apart, well within the resolution of the vertex tracker. The relative $B$ decay times may then be determined and used to measure the time dependence of the decay rates.

The interaction region, where the two beams are focussed such that the $e^{+}$and $e^{-}$ have head-on collisions, is realized using an array of quadrupole magnets acting near the interaction point (IP). There is an offset of $20 \mathrm{mrad}$ between the collision axis and the $z$-axis of the BABAR detector in the horizontal plane to minimize the perturbation of the beams by the solenoidal field. The two beams are then separated after the collision within $62 \mathrm{~cm}$ of the IP by dipole magnets B1, located $21 \mathrm{~cm}$ away from either side of the IP, to avoid spurious collisions between out of phase bunches.

The energy of the two particle beams is calculated from the total magnetic bending strength and the average deviation of the accelerating frequency from the central value. The systematic error on the PEP-II calculation of the absolute beam energies is estimated 


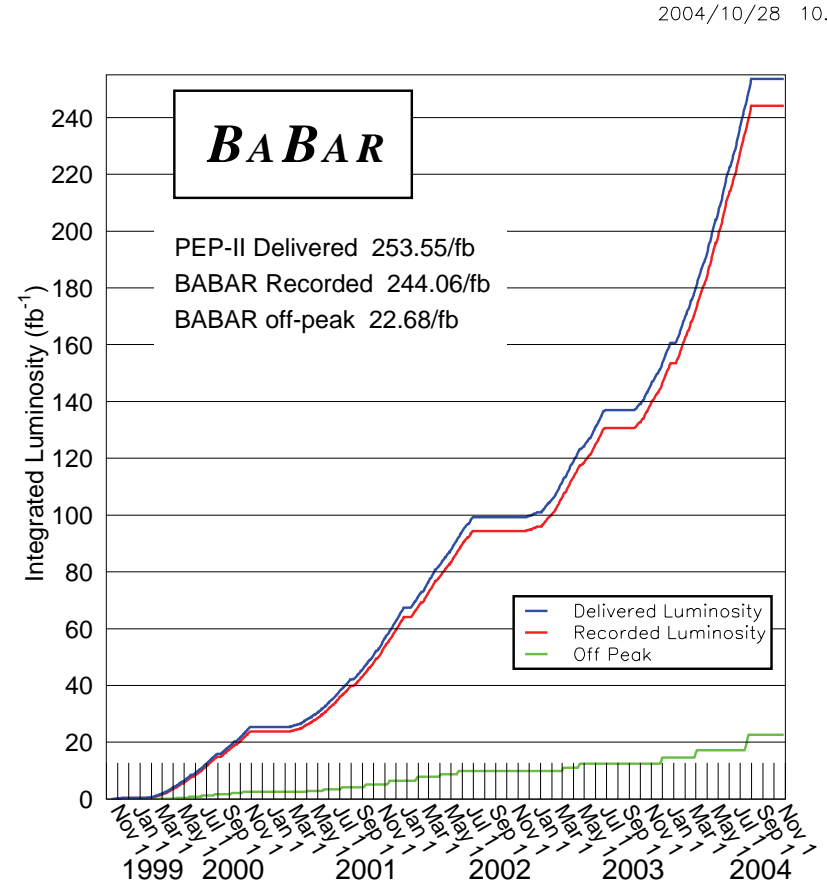

Figure 3.3: Integrated luminosity delivered by the PEP-II collider and recorded by the $B A B A R$ detector. Note that this analysis is based on Run-1 and Run-2 data taking periods (before November 2002), which contains about 88 million $B \bar{B}$ pairs.

to be $5-10 \mathrm{MeV}$, while the relative energy setting for each beams is accurate and stable to about $1 \mathrm{MeV}$. Most of the data is recorded at the $\Upsilon(4 S)$ energy. In order to evaluate the underlying continuum ${ }^{2}$ listed in table 3.1 , about $10 \%$ of the data is collected with a center of mass energy $40 \mathrm{MeV}$ below the $\Upsilon(4 S)$ resonance.

The instanteneous luminosity is measured online by PEP-II using radiative Bhabha scattering, whereas the absolute luminosity is derived offline from other QED processes. The systematic uncertainty on the absolute value of the luminosity is estimated to be about $1.1 \%$, as discussed in section 7.13. PEP-II reached the designed peak luminosity of $3 \times 10^{33} \mathrm{~cm}^{-2} \mathrm{~s}^{-1}$ in its first year of running and in late 2004, an instanteneous luminosity $\sim 10^{34} \mathrm{~cm}^{-2} \mathrm{~s}^{-1}$ was achieved. Figure 3.3 shows the luminosity delivered by the PEP-II collider and recorded by the BABAR detector since the beginning of operation in November 1999. The data is

\footnotetext{
${ }^{2}$ Events other than $e^{+} e^{-} \rightarrow \Upsilon(4 S)$.
} 
divided in four runs, corresponding to data taken between extended shutdown periods. So far, about $250 \mathrm{fb}^{-1}$ of data has been recorded by the BABAR detector, which corresponds to $\sim 230$ million $B \bar{B}$ pairs. Note that the analysis presented in this dissertation is based on Run-1 and Run-2 data taking periods (before November 2002).

\subsection{The tracking system}

The BABAR tracking system consists of two subdetectors: the silicon vertex tracker and the drift chamber. The main purpose of this system is the detection of charged particles and the measurement of their momentum and angles with high precision for the transverse momentum range $60 \mathrm{MeV} / c<p_{t}<4 \mathrm{GeV} / c$. A superconducting solenoid, located between the EMC and the IFR, provides the necessary magnetic field of 1.5 tesla. The DCH provides most of the momentum measurements, but the SVT is the sole tracking device for very low- $p_{t}$ charged particles. Fiducial information gathered by the tracking system is also important for track extrapolations to the DIRC, EMC and IFR. Most critical are the angle measurements at the DIRC, because the uncertainties in the charged particle track parameters add to the uncertainty in the measurement of the Čerenkov angle. Thus, the resolution from the combined SVT and DCH measurements should be of the order of $1 \mathrm{mrad}$, i.e. smaller than the average DIRC Čerenkov angle measurement.

\subsubsection{The silicon vertex tracker}

The silicon vertex tracker is designed to provide the reconstruction of charged particle trajectories and decay vertices near the interaction point. $C P$ violation studies require a precise reconstruction of each $B$ meson decay vertex along the $z$ axis with a resolution of better than 80 microns. The experiment also relies on vertexing to discriminate between beauty, charm, and light-quark vertices. Because of the presence of the 1.5 tesla solenoidal magnetic field, the SVT must provide stand-alone tracking for particles with a transverse momentum less than $120 \mathrm{MeV} / c$, the minimum that can be measured in the DCH alone. To accomplish this requirement, the number of impact points of a single charged particle must 


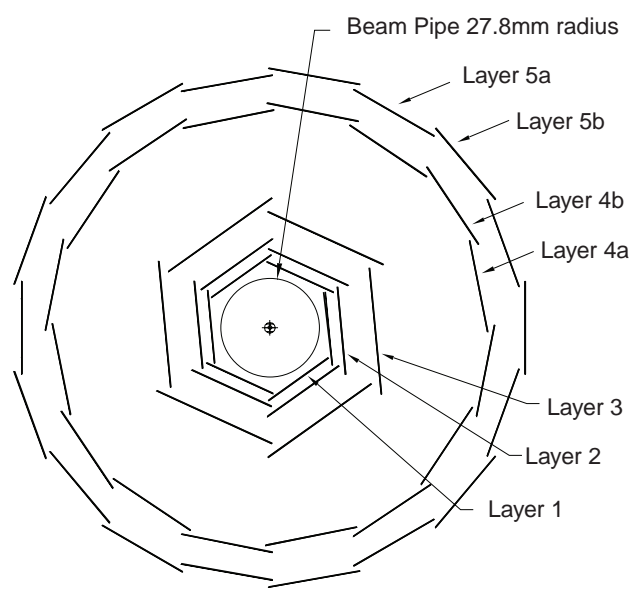

Figure 3.4: Cross-section of the SVT in a plane perpendicular to the beam axis ( $x-y$ plane). The first layer radius is $3.3 \mathrm{~cm}$, and the maximum fifth layer radius is $14.4 \mathrm{~cm}$ [44].

be greater than three. This feature is critical in order to fully constrain the decay products of the $B$ mesons. Beyond the standalone tracking capability, the SVT must provide the best measurement of track angles, which is critical to achieve the design resolution for the Čerenkov angle for high momentum tracks.

Because of its proximity to the beam pipe, the SVT has been designed to withstand high levels of radiation: an integtrated dose of about 2 MRad of ionizing radiation. The expected radiation dose is $1 \mathrm{Rad} /$ day in the horizontal plane immediately outside the beam pipe, and $0.1 \mathrm{Rad} /$ day on average otherwise. A radiation monitoring system capable of aborting the beams ensures that the device is not exposed to radiation beyond the designed tolerance. Since the SVT is inaccessible during normal detector operations, reliability and robustness are essential.

These guidelines have led to the design of the silicon vertex tracker. The SVT, located inside the support tube of the beam magnets, covers the polar angle region between $20.1^{\circ}$ and $150.2^{\circ}$ from the beam line, the region at smaller polar angles being occupied by the B1 permanent dipole magnets as shown in figure 3.1. Figure 3.4 displays the layout of the SVT in the $x-y$ plane. It consists of five concentric cylindrical layers of double- 


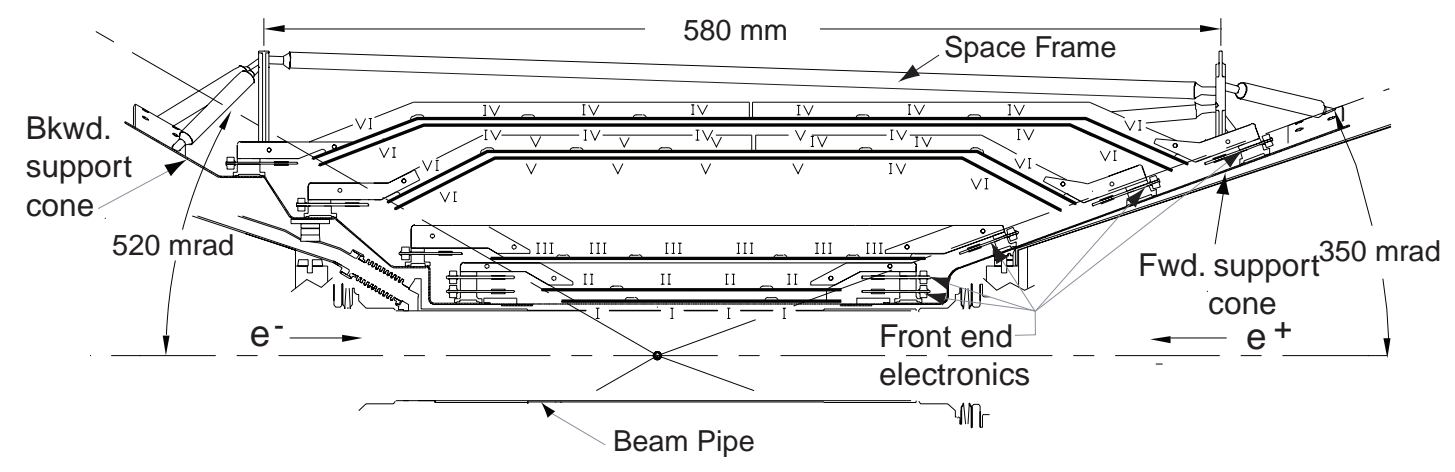

Figure 3.5: Longitudinal view of the upper half of the SVT. The outer layers have an arched structure to minimize the amount of silicon needed for the solid angle coverage and to reduce large incidence angles [44].

$$
\text { :- }
$$

sided silicon strip sensors with radii between $32 \mathrm{~mm}$ for the innermost and $144 \mathrm{~mm}$ for the outermost layer. The silicon strip sensorế âre double-sided to reduce the amount of material the particles have to go through, and hence minimize energy loss and multiple scattering probability compared to single-sided detectors. '- I'

As shown in figure 3.4, each layer is divided in azimuth into modules, which are arranged in a way such that neighbouring ones overlap each other, ensuring a full azimuthal coverage. The three inner layers comprise six barrel-shaped detector modules and are

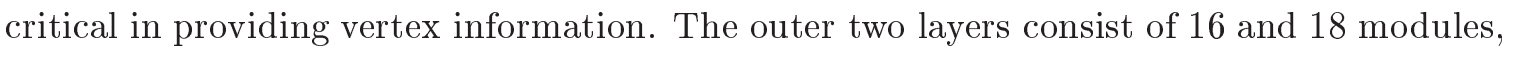
respectively, and follow an arched structure to minimize the amount of silicon needed for the solid angle coverage and to reduce large incidence angles. They provide tracking information and pattern recognition which can be used in conjonction with other subdetectors or in stand-alone mode.

The SVT five layers contain 340 silicon strip detectors, the equivalent of a total silicon area of $0.94 \mathrm{~m}^{2}$, and count 150,000 readout channels. The inner sides of the detectors have strips oriented perpendicular to the beam direction to measure the $z$ coordinate, whereas the outer sides, with longitudinal strips, allow the $\phi$ coordinate measurement. The readout pitch in $\phi$ varies between 50-100 microns, and between 100-210 microns in $z$ for 

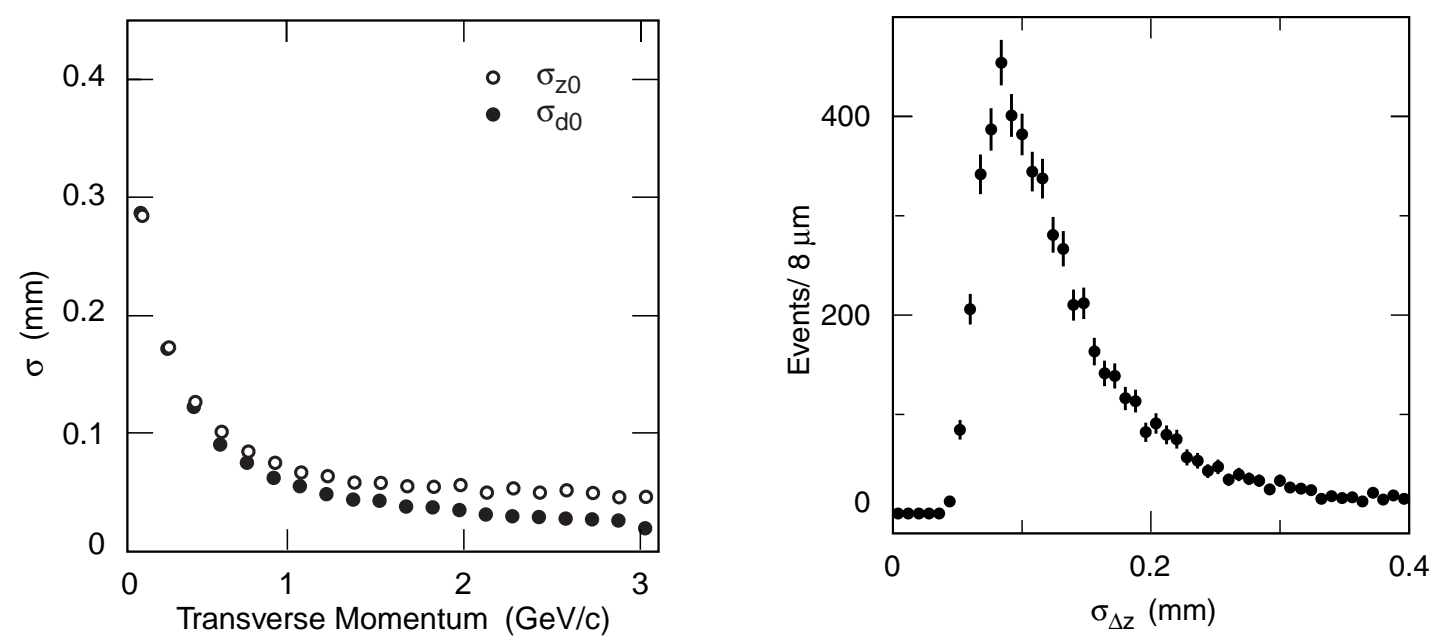

Figure 3.6: Resolution in the parameters $d_{0}$ and $z_{0}$ for tracks as a function of the transverse momentum (left), and error distribution for the measurement of the separation between two $B$ meson decay vertices [45].

the inner/outer-most layer, respectively. These characteristics lead to an intrinsic one-point resolution of about 10 microns in $\phi$ and $12-25$ microns in $z$.

The SVT efficiency may be calculated by comparing the number of associated hits to the number of tracks crossing the active area. The combined hardware and software efficiency as a function of the track incident angle and for each of the five layer is about 97\%, beyond the design goals [45]. Single hit resolutions are in excellent agreement with Monte Carlo simulations. Figure 3.6 displays the resolution of the reconstructed track parameter $d_{0}$ and $z_{0}$, which represent the distances between the point of closest approach to the $z$-axis and the origin of the $x-y$ plane and along the $z$ axis, respectively. Also shown is the error distribution for the measurement of the separation between two $B$ meson decay vertices.

\subsubsection{The drift chamber}

The reconstruction of charged particles with transverse momenta greater than $120 \mathrm{MeV} / c$ is the main task of the drift chamber. The DCH, displayed on figure 3.7 , is a $280 \mathrm{~cm}$ long cylinder with an inner radius of $26.6 \mathrm{~cm}$ and an outer radius of $80.9 \mathrm{~cm}$. Due to 


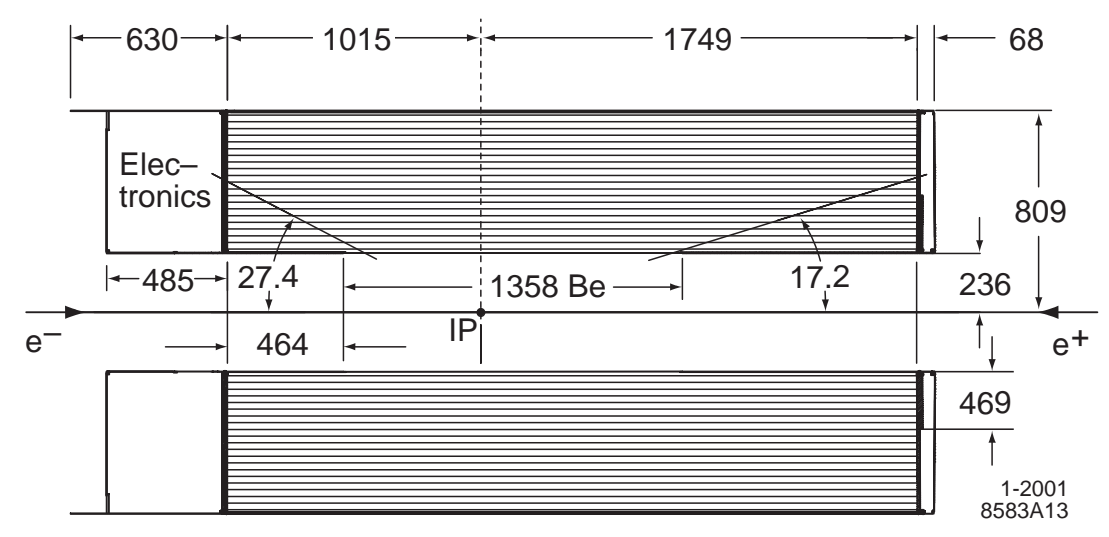

Figure 3.7: Longitudinal cross-section of the DCH [44].

the asymmetric beam energies along the $z$ axis, the center is offset by $37 \mathrm{~cm}$ from the interaction point, leading ta a a chamber operates with a low density gas mixture of helium (80\%) and isobutane (20\%) to reduce multiple scattering.

The sense wires consist of gold plated tungsten-rhenium wires with a diameter of 20 microns. Each of these is surrounded by six $80-120 \mu \mathrm{m}$ field wires made of aluminum making up a hexagonal structure called the drift cell. A schematic layout of the innermost drift cells is shown in figure 3.8. The drift chamber contains 7104 cells arranged in 40 cylindrical layers, with four layers being grouped into one superlayer. In order to reconstruct the 3 dimensional trajectory of a particle, the various superlayers have wires with different stereo angles: axial (A), positive (U) and negative (V) stereo angles. The arrangement of the ten superlayers follows the pattern AUVAUVAUVA, with stereo angles increasing radially from $\pm 45 \mathrm{mrad}$ to $\pm 76 \mathrm{mrad}$. The cells are designed to provide an average intrinsic spatial resolution of aboūt tit 40 microns.

The field wires are grounded while the sense wires were designed to operate at 1960 V. Unfortunately, an accident happened during the commissioning phase which required to lower the voltage to $1900 \mathrm{~V}$ out of concern for a small region of the chamber that was damaged. Aging studies hinted that a voltage of $1930 \mathrm{~V}$ was acceptable and the drift 
- i

i'

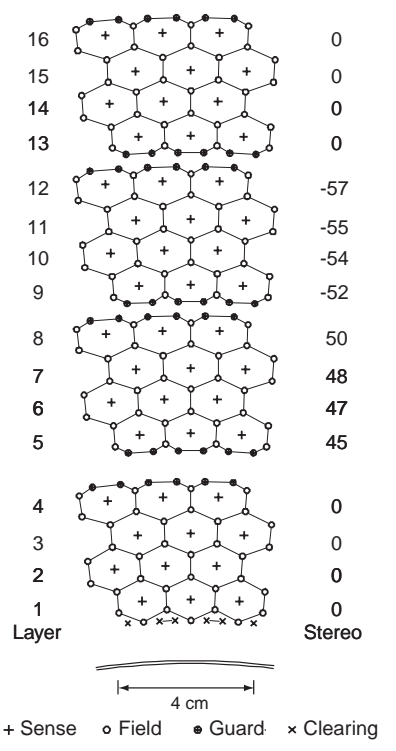

Figure 3.8: Schematic layout of drift cells for the four innermost superlayers [44]. Lines have been added between field wires to aid in visualization of the cell boundaries. On the left are shown two sets of drift cell isochrones, i.e., countours of equal drift times of ion in cells of layers 3 and 4 . The isochrones are spaced by $100 \mathrm{~ns}$.

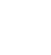

charber has been operating at this lower value since January 2001. Displayed on figure 3.8 :i is a diagram of two sets of drift cell isochrones for cells in layers 3 and 4 . The isochrones are circular near the sense wires, but deviate greatly from circles near the field wires. Ions originating in the gap between superlayers are collected by cells in the edge layers after a delay of several microseconds. These lagging ions do not affect the drift times measurements, but they contribute to the $\mathrm{dE} / \mathrm{dx}$ measurement.

The drift chamber measurement of the energy loss per unit distance is performed from the charge collected within each cell, and is an important information for particle identification as shown in figure 3.9. The charge collected per signal cell is measured as part of the feature extraction algorithm. The truncated mean of the specific energy loss of a track is computed from, the lowest $80 \%$ of the individual (hit) measurements. Corrections need to be applied for:

1) Changes in gas pressure and/or temperature. Such changes can affect $\mathrm{dE} / \mathrm{dx}$ by as 


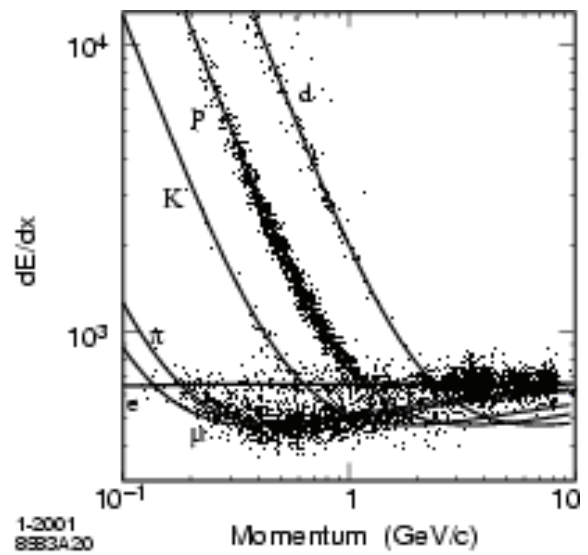

Figure 3.9: Particle identification via $\mathrm{dE} / \mathrm{dx}$ measurements in the drift chamber [44].

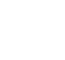

much as $\pm 9 \%$. These changes are corrected by a single overall multiplicative constant (referred to as the global gas gain) online;

2) Differences in cell geometry and charge collection. These lead to $\pm 8 \%$ variation and are corrected by a set of multiplicative constants for each wire;

3) Charge saturation. The build up of charges within the drift cell cause screening and lead to $\pm 11 \%$ variation in $\mathrm{dE} / \mathrm{dx}$. It is corrected by a second-order polynomial in the dip angle, $\lambda$;

4) Non-linearities at large dip angles. Corrections of about $\pm 2.5 \%$ are done using a sixth-order Chebyshev polynomial as a function of $\lambda$;

5) Non-linearities with respect to the entrance angle $(\phi)$ and the distance of closest approach (DOCA). In fact, experimental data shows a correlation between the variations in $\mathrm{dE} / \mathrm{dx}$ and $\phi$ and DOCA.

Correction for (1) is computed online whereas (2-5) must be studied offline. The mean rate of energy loss of particles $(\mathrm{dE} / \mathrm{dx})$ travelling through the $\mathrm{DCH}$ is given by the 
Bethe-Bloch equation. In BABAR, it is parameterized as

$$
\frac{\mathrm{dE}}{\mathrm{dx}}=\frac{P_{1}}{\beta^{P_{5}}}\left(P_{2}-\beta^{P_{5}}-\log \left(P_{3}+(\beta \gamma)^{P_{4}}\right)\right)
$$

where $\beta \gamma$ is measured as $p / m$, and $\left\{P_{1}, P_{2}, P_{3}, P_{4}, P_{5}\right\}$ are free parameters; this function is universal and is used for all particles. In order to parameterize $\mathrm{dE} / \mathrm{dx}$, the mean $\mathrm{dE} / \mathrm{dx}$ is

computed in bins of $\beta \gamma$ for different particle species: proton, pion, muon and electrons. The resolution may then be computed from the difference between the measured and calculated $\mathrm{dE} / \mathrm{dx}$ and result in about $7.3 \%$ for electrons, in agreement with the design values. Details concerning the $\mathrm{dE} / \mathrm{dx}$ calibration may be found in [46].

When operating at the design luminosity, the PEP-II beam crossing rate is $4.2 \mathrm{~ns}$ such that it is impossible to tell, in real time, which event in the detector arose from which beam crossing. Information from the DCH is therefore used to determine this. Using track segments found independently in each superlayer, it is possible to relate the $\Delta \mathrm{T} 0$ to the time of the interaction producing the particle. The time-of-flight of the particle must be accounted for in this procedure. The resulting resolution on the collision time is on the order of $\sim 1$ ns for a typical event.

Overall, the DCH meets the design expectations. It contributes primarily to the measurement of the transverse momentum with a resolution of

$$
\frac{\sigma_{p_{t}}}{p_{t}}=(0.13 \pm 0.01) \% \cdot p_{t}+(0.45 \pm 0.03) \%
$$

where the momentum is measured in units of $\mathrm{GeV} / c$.

\subsection{The Čerenkov light detector}

The detector of internally reflected Čerenkov light is designed to achieve hadronic particle identification, in particular pion/kaon separation up to $4 \mathrm{GeV} / c$. It is based on the principle that charged particles traversing a medium radiate if their velocity is greater than the local phase velocity of light. The light is emitted at a certain Čerenkov angle, $\cos \theta_{c}=1 / n \beta$, which depends on the index of refraction $n$ of the medium and the velocity of the particle 


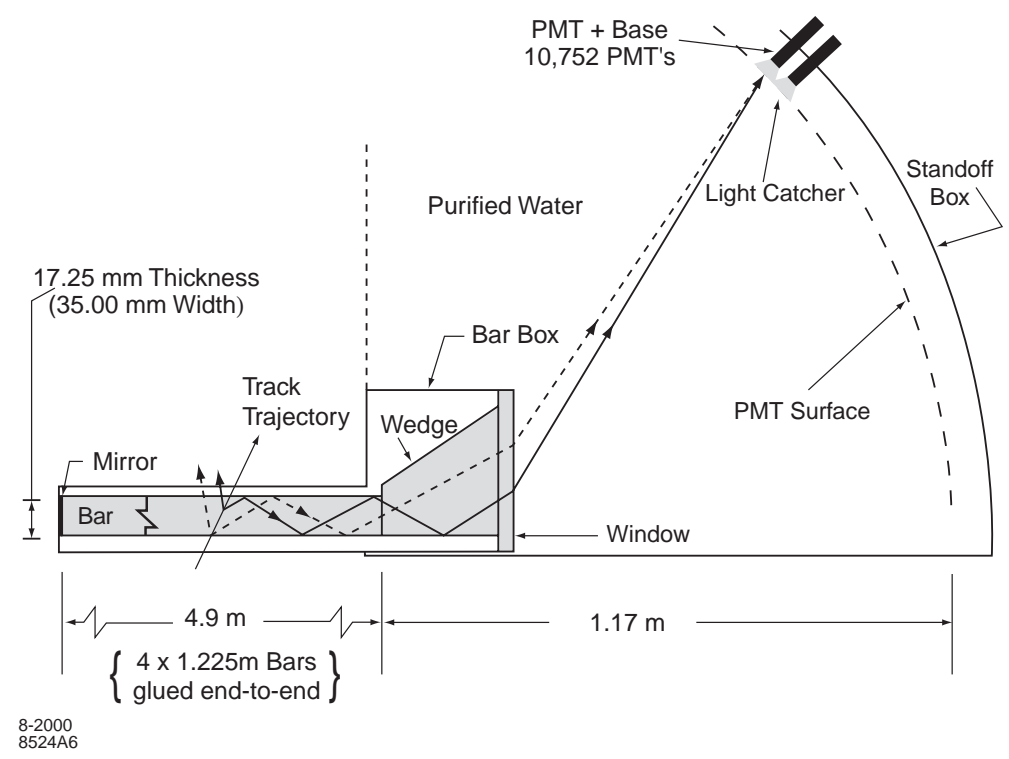

Figure 3.10: Schematic of the DIRC displaying a fused silica bar (grey), standoff box (white) and imaging region (dashed line) [44].

$\beta=v / c$. The measurement of $\theta_{c}$ can then be used in conjunction with tracking information to identify particles.

The DIRC features a new type of ring imaging Čerenkov detector based on total internal reflection. Figure 3.10 illustrates the principle of light production, transportation and detection. The radiator is located between the drift chamber and the electromagnetic calorimeter, and extends $178 \mathrm{~cm}$ forward from the interaction point. It consists of 144 thin fused silica (quartz) bars arranged in a 12-sided polygon barrel of $84 \mathrm{~cm}$ radius. Each bar has dimension $1.76 \mathrm{~cm} \times 3.5 \mathrm{~cm} \times 1.225 \mathrm{~m}$, and has a refractive index of about 1.47 . The bars are glued end-to-end in groups of $3 \times 4$ and are housed in bar boxes surrounded by nitrogen of refractive index of $\approx 1$. At normal incidence, this corresponds to $19 \%$ of a radiation length. Due to gaps between the boxes, the azimuthal coverage is $93 \%$, and about $87 \%$ of the polar solid angle in the center-of-mass frame is covered.

Since the refractive index of the bars is larger than $\sqrt{2}$, a fraction of the Cerenkov 
light emitted by charged particles traversing the radiator propagates via total internal reflection along the bar, regardless of the incidence angle of the tracks. Only the rear end of the detector is instrumented, so a mirror of $92 \%$ reflectivity is located at the front end of the bar. Hence, the light traveling through the radiator cannot leave the bars until the rear end of the detector is reached after multiple internal reflections. Photons exciting the bars at large angles in the radial direction are partly recovered into the instrumented area by a prism at the readout end. The prism also reduces the required area for photon detection by a factor of two.

The photons eventually exit the prism at the rear end of the bars into an expansion region filled with 6,000 litres of purified water: the standoff box. At the backplane of the standoff box, the photons are detected by a close packed array of 10,572 photomultiplier tubes (PMTs) of approximately $25 \%$ detection efficiency at $400 \mathrm{~nm}$, pointing to the exit of a corresponding bar box. Each PMT has a diameter of $2.82 \mathrm{~cm}$ and is operated directly in water, with an average distance of $1.2 \mathrm{~m}$ to the end of the fused silica bars. Except for a number of discrete ambiguities, the magnitude of the Cerenkov angle is preserved during this process. To resolve these ambiguities, measurements of the photon arrival time and pattern recognition algorithms are used.

The DIRC is primarily limited by track reconstruction. Nevertheless, it achieves a single photon resolution of about 10.2 mrad. The resolution on a track scales as $\sigma_{\gamma} / \sqrt{N_{\gamma}}$, where $\sigma_{\gamma}$ is the resolution of a single photon, and $N_{\gamma}$ is the number of detected photons, about 30 for tracks entering the radiator at normal incidence. Di-muon studies yield an average track resolution of about $2.5 \mathrm{mrad}$, which corresponds to a $4.2 \sigma$ separation between kaons and pions at $3 \mathrm{GeV} / c$.

\subsection{The electromagnetic calorimeter}

The electromagnetic calorimeter is designed to measure electromagnetic showers over a wide energy range, from $20 \mathrm{MeV}$ to $9 \mathrm{GeV}$. It is the principal device for electron/pion separation, 


\begin{tabular}{ll}
\hline Parameter & Values \\
\hline \hline Radiation length & $1.85 \mathrm{~cm}$ \\
Molière radius & $3.8 \mathrm{~cm}$ \\
Density & $4.53 \mathrm{~g} / \mathrm{cm}^{3}$ \\
Light yield & $50,000 \mathrm{\gamma} / \mathrm{MeV}$ \\
Peak emission $\left(\lambda_{\max }\right)$ & $565 \mathrm{~nm}$ \\
Refractive index at $\lambda_{\max }$ & 1.80 \\
\hline
\end{tabular}

Table 3.2: Properties of $\mathrm{CsI}(\mathrm{Tl})$.

photon and neutral pion reconstruction. The calorimeter consists of 6580 thallium-doped caesium iodide $(\mathrm{CsI}(\mathrm{Tl}))$ crystals which feature a small Molière radius $\left(R_{m}=3.8 \mathrm{~cm}\right)$ and a short radiation length $(1.85 \mathrm{~cm})$ for precise angular measurements and which fully contain showers with a relatively compact design. Furthermore, the high light yield of CsI(Tl) and the emission spectrum permit efficient use of silicon photodiodes which operate well in high magnetic fields. Table 3.2 summarizes some of the physical properties of the material used for the crystals.

As shown on figure 3.11, the EMC crystals are arranged in a cylindrical barrel section $\left(26.9^{\circ}<\theta<140.8^{\circ}\right)$ and a forward conic endcap $\left(15.8^{\circ}<\theta<26.9^{\circ}\right)$. Located within the magnet cryostat, the barrel has an inner radius of $91 \mathrm{~cm}$ and an outer radius of $136 \mathrm{~cm}$. It consists of 48 polar angle rows, each having 120 identical crystals in azimuthal angle. The arrangement is different in the endcap, where 820 crystals are divided into 8 polar angle rows with a segmentation in $\phi$ varying between 80 and 120 crystals. To account for the boost, which leads to higher energies at small polar angles, the crystal lengths increase from $29.76 \mathrm{~cm}\left(15.1 X_{0}\right)$ in the backward direction to $32.55 \mathrm{~cm}\left(17.6 X_{0}\right)$ in the endcap. The typical area of the front face is $4.7 \times 4.7 \mathrm{~cm}^{2}$, while the back face area is typically $6.1 \times 6.0 \mathrm{~cm}^{2}$.

The crystals act not only as a total-absorption scintillating medium, but also as a light guide to collect light at the photodiodes. Depicted in figure 3.12 is the assembly of a crystal. Mounted at the back of the crystal are two silicon photodiodes collecting 


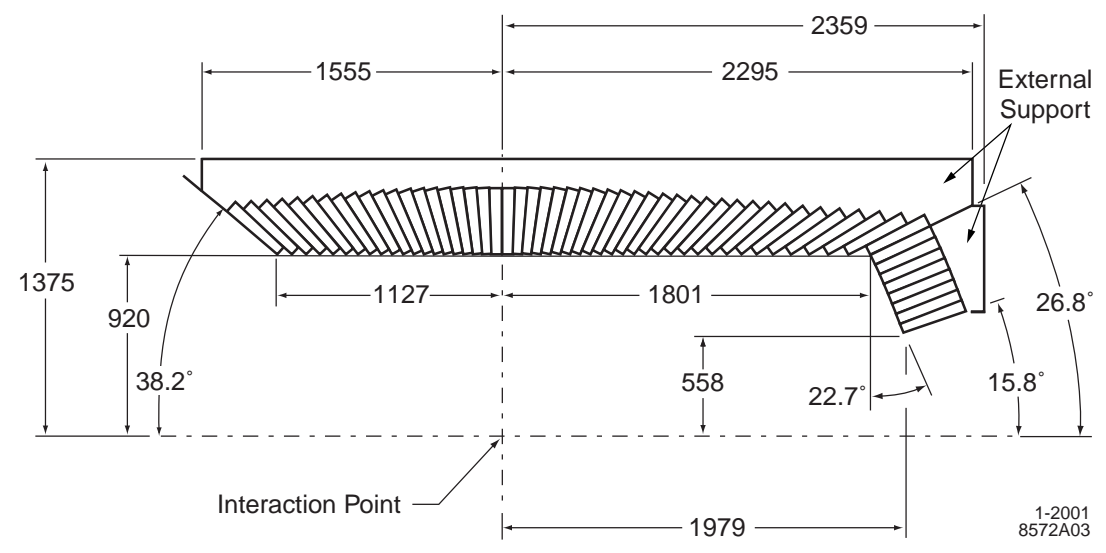

Figure 3.11: Longitudinal cross-section of the EMC showing the dimensions of the calorimeter barrel and forward endcap [44].

scintillation light. The crystal is surrounded by a reflector plate to prevent, the loss of photons missing the diodes. Preamplifiers are connected to each diode, and the whole - :

readout system is enclosed within aluminum housing to provide shielding against the RF noise. At the polished surface of the crystal, the light is internally reflected and only a small fraction of it is transmitted. The latter is partially recovered by two layers of a diffuse white reflector (Tyvek) wrapped around each crystal. The crystal are held by modules made of carbon-fiber composit with 300 micron walls between the individual compartments. Althogether, the inactive material between the crystals leads to gaps of about $1.25 \mathrm{~mm}$. To

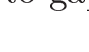

minimize the loss of photons entering the EMC at those gaps, the crystal axes feature a non-projectivity in $\theta$. The average light yield is about 7300 photo-electrons/MeV.

Since the energy deposited by a particle is scattered over many crystals, a clustering algorithm is applied. Starting with a seed crystal whose energy measurement exceeds a threshold of $5 \mathrm{MeV}$, all neighboring crystals with energy deposition above $1 \mathrm{MeV}$ are associated to the seed. This procedure is repeated iteratively for each of these neighbors, leading to a set of adjacent clusters, which represents the energy deposited by one or more particles. If the energy distribution within the cluster displays more than one local maximum, it is divided into as many "bumps", with each "bump" assumed to represent the 


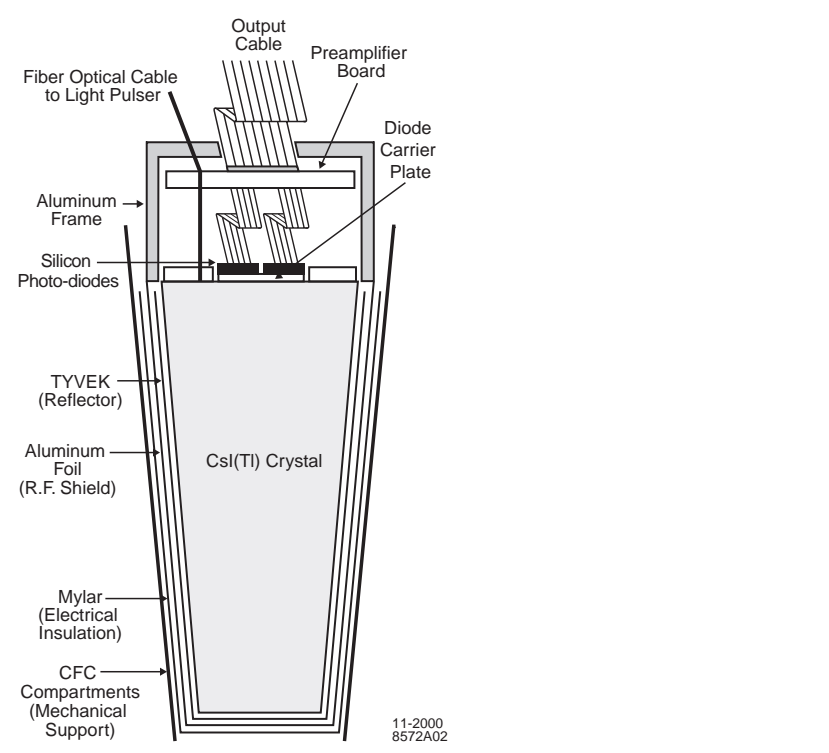

Figure 3.12: Schematic view of an EMC crystal assembly [44].

energy deposited by a single particle. Clusters with one local maximum are also referred to as a bump.

The calorimeter has an efficiency of about $96 \%$ for detecting photons with energy above $20 \mathrm{MeV}$, backgrounds and the amount of material between the IP and the calorimeter being the major limiting factors. The EMC angular resolution is determined by the transverse crystal size and the distance from the interaction point. The photon resolution was studied using $\pi^{0}$ decays. As shown in figure 3.13, it varies between $12 \mathrm{mrad}$ for low energy photons, to $3 \mathrm{mrad}$ for very energic photons. A fit to an emperical function yields

$$
\sigma_{\theta}=\sigma_{\phi}=\left(\frac{3.87 \pm 0.07}{\sqrt{E(\mathrm{GeV})}} \oplus(0.00 \pm 0.04)\right) \operatorname{mrad},
$$

where $E$ is the energy of the photon in $\mathrm{GeV}$.

The EMC energy resolution was obtained using a radioactive source yielding $\sigma_{e} / E=$ $(5.0 \pm 0.8) \%$ at $6.13 \mathrm{MeV}$. For high energies, the resolution was studied using Bhabha scattering events, where the energy of the detected shower can be predicted from the polar angle of the electrons. Figure 3.13 shows the energy resolution extracted from a variety of 

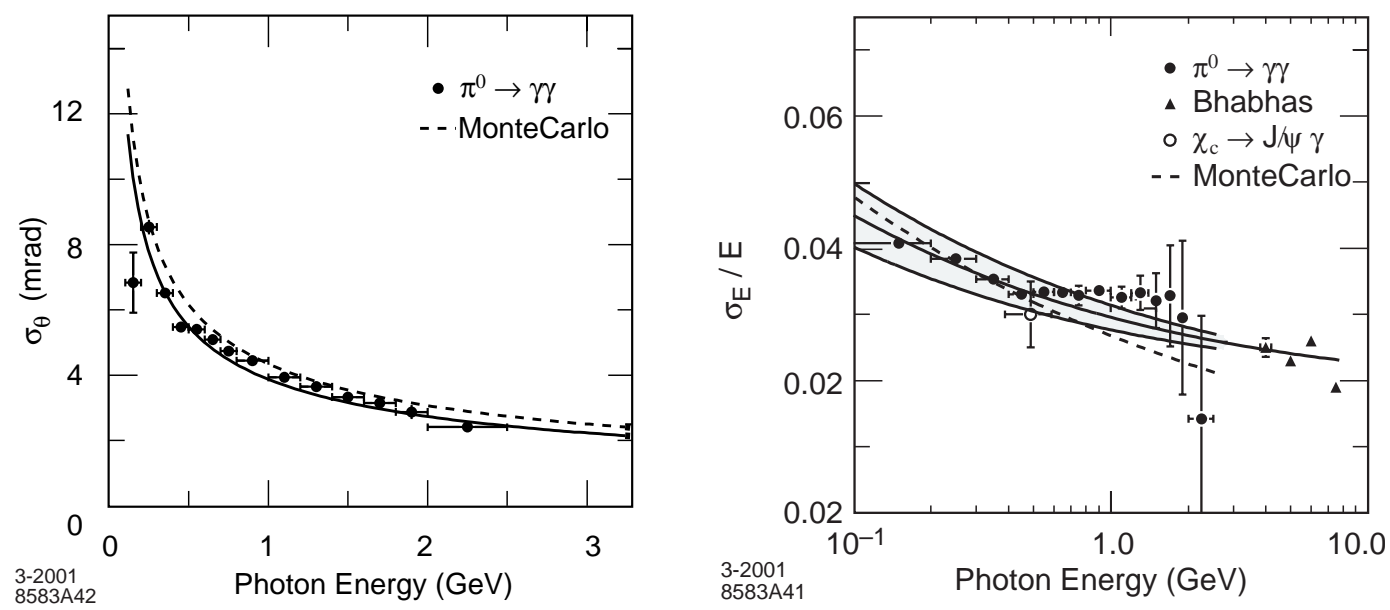

Figure 3.13: Electromagnetic calorimeter performance. The spatial (left) and energy (right) resolutions are pilotted as a function of the incident photon energy [44].

processes as a funcion of energy. A fit to the energy dependence results in

$$
\quad \frac{\sigma_{E}}{E}=\left(\frac{2.32 \pm 0.30}{4 \sqrt{E\left(\mathrm{G}^{\top} \mathrm{V}^{\top}\right)}} \oplus(1.85 \pm 0.12)\right) \%
$$

At lower energies, the resolution is dominated by fluctuations in photon statistics and by beam generated backgrounds. Above $1 \mathrm{GeV}$, the resolution depends mostly on the nonuniformity in light collection from leakage or absorption in the material between or in front of the crystals. Although the resolution parameters obtained are higher than the design expectations, they agree well with Monte Carlo simulations which include the contributions from electronic noise and beam background, as well as the ing pact of the material and the energy thresholds.

\subsection{The instrumented flux return}

The instrumented flux return is designed to identify muons and to detect neutral hadrons using the steel flux return of the magnet. The IFR, depicted on figure 3.14, consists of a barrel and two endcaps made of iron, and covers the polar angle region between $17^{\circ}$ and $157^{\circ}$. The steel is segmented into 18 plates, increasing in thickness from $2 \mathrm{~cm}$ in the inner 


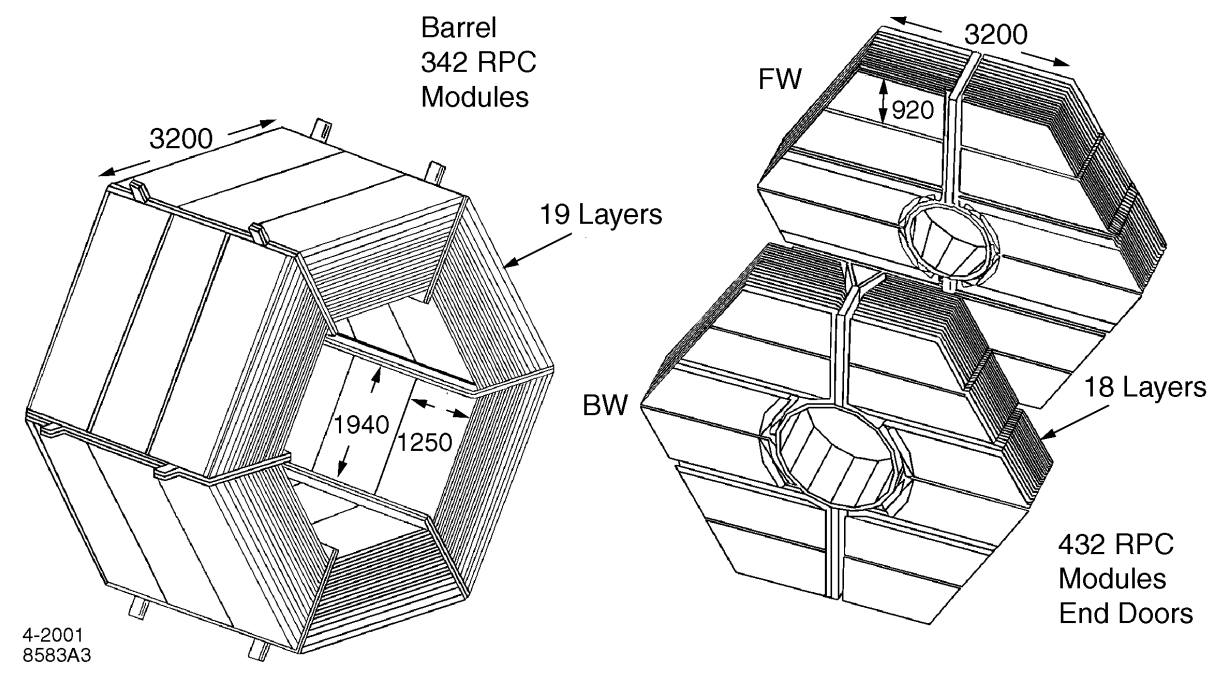

Figure 3.14: View of the IFR barrel and endcap wheels [44].

nine plates to $10 \mathrm{~cm}$ for the outermost plates. This feature was motivated by Monte Carlo studies which demonstrated that thin absorber plates improve muon and $K_{L}^{0}$ detection during the first absorption length only, therefore allowing wider absorbers and less readout layers at larger distances. The total thickness of the iron plates at normal incidence amount to $65 \mathrm{~cm}(60 \mathrm{~cm})$ in the barrel (endcaps).

The IFR is instrumented with 806 resistive plate chambers (RPCs) mounted in the spaces between the steel plates. The gap beteen the steel plates is $3.5 \mathrm{~cm}$ in the inner layers of the barrel and $3.2 \mathrm{~cm}$ elsewhere. There are $19 \mathrm{RPC}$ layers in the barrel, and 18 in the endcaps. The barrel contains two additional layers of cylindrical RPCs located between the EMC and the magnet cryostat to detect particles exiting the EMC.

Although they differ in details, planar and cȳindrical RPCs are based on the same concept. They are gas chambers enclosed between bakelite (phenolic polymer) sheet $2 \mathrm{~mm}$ thick surrounding a $2 \mathrm{~mm}$ gap filled with a gas mixture of $56.7 \%$ argon, $38.8 \%$ Freon-134a and $4.5 \%$ isobutane. A cross section of an RPC is shown schematically in figure 3.15 . The external surfaces of the bakelite are coated with graphite to achieve a high surface resistivity, whereas the inner surfaces are treated with linseed oil. The two graphite surfaces 


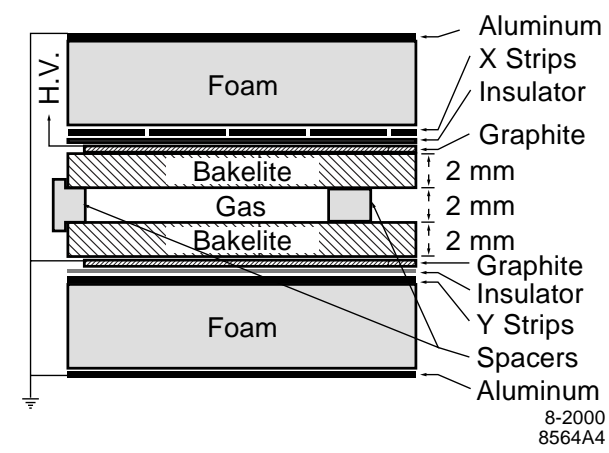

\section{1}

Figure 3.15: Cross-section of a RPC from the IFR [44].

$$
\text { i: }
$$

are connected to a high voltage of about $8 \mathrm{kV}$ and to ground, and both are protected by an insulating mylar film. Hence, ionizing particles crossing the gas gap will produce a discharge. This ionization induces a pulse which is picked up by aluminum strip electrodes running in perpendicular directions on the outside of the bakelite. The bakelite was chosen for its resistive properties $\left(10^{11}-10^{12} \Omega \mathrm{cm}\right)$ which localize the discharge.

IFR muon identification is based on measuring the number of traversed interaction lengths in the entire detector and comparing it with the number of expected interaction lengths for a muon of a given momentum. Moreover, the projected intersections of a track with the RPC planes are computed and must be associated within a certain transverse distance from the extrapolated trajectory of a reconstructed charged track. Finally, the average number of excited strips per layer is expected to be larger for pions producing a hadronic shower than for muons. Using the above observations, the muon identification efficiency was measured using $e^{+} e^{-} \rightarrow \mu^{+} \mu^{-}(\gamma)$ events and two photon production of $\mu^{+} \mu^{-}$ pairs, and resulted in an overall efficiency of about $90 \%$ in $1999,70 \%$ in 2000 , and $60 \%$ in -2001.

The efficiency drop is caused by a large fraction of the RPC modules having suffered high temperatures during the summer of 2000. In fact, the experimental hall does not have a temperature regulation system and the temperature inside the steel rose to more than $37^{\circ} \mathrm{C}$. Although the exact problem is not understood, it seems that high temperatures 
damaged the RPCs: change in the bakelite bulk resistivity, loosened spacers, gas flow, or gas composition. A number of prototype RPCs developed similar efficiency problems after being opeated above $36^{\circ} \mathrm{C}$ for a period of two weeks. A water cooling system was added on the steel, stabilizing the temperature below $24^{\circ} \mathrm{C}$ throughout the IFR, but the efficiency of some of the RPCs continued to deteriorate while others remained stable.

\subsection{The trigger system}

The trigger system is responsible for selecting events of interest while rejecting background events, and keeping the event rate at a reasonable level, about $120 \mathrm{~Hz}$ at design luminosity. The total trigger efficiency is required to exceed $99 \%$ for all $B \bar{B}$ events and at least $95 \%$ for continuum events. The trigger is implemented as a two-level hierarchy: level 1 (L1) in hardware followed by level 3 (L3) in software. The trigger system is designed to accommodate up to ten times the expected PEP-II background rates at design luminosity and to degrade slowly above that level.

\subsubsection{L1 trigger}

The decision to read out the front-end data from the detector subsystems is made by the fast control and timing system (FCTS) based primarily on information from the level 1 trigger system. The L1 trigger decision is based on charged tracks in the DCH above a preset transverse momentum, showers in the EMC, and tracks detected in the IFR. As such, the L1 is broken into three trigger subsystems.

The input data to the drift chamber trigger consists of one bit for each of the 7104 DCH cells, updated every 269 ns. These bits convey time information derived from the sense wire signal for that cell. Track segments are formed from clusters of cell hits, and their $\phi$ positions and drift time estimates are then used to assemble the segments into full tracks. The drift chamber trigger then checks those tracks against preset transverse momentum

thresholds. For tracks originating from the IP, the efficiency for finding track segments is 97\%. The DCH trigger efficiency is above $90 \%$ for tracks with $p_{t}>1 \mathrm{GeV} / c$. 


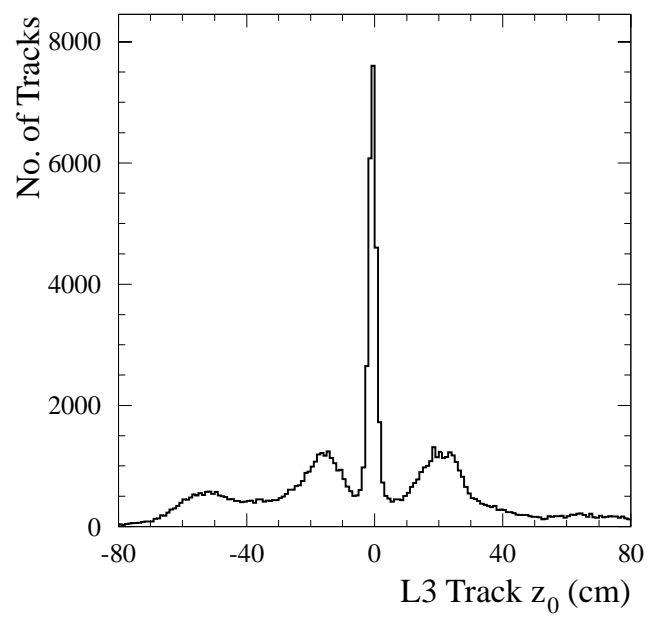

Figure 3.16: Distribution of $z_{0}$ for tracks satisfying the L1 trigger as reconstructed by L3 [44].

For trigger purposes, the EMC barrel is divided into 240 towers of $8 \times 3(\theta \times \phi)$ crystals, whereas the endcap is divided into 40 towers, each forming a wedge in $\phi$ containing 19-22 crystals. For each tower, all crystal energies above a threshold of $20 \mathrm{MeV}$ are summed and sent to the calorimeter trigger every $269 \mathrm{~ns}$. The trigger identifies energy deposits that span two adjacent towers and combines them when necessary. The EMC trigger efficiency is $99 \%$ for clusters of energy above $180 \mathrm{MeV}$, close to the average energy deposition of a minimum ionizing particle at normal incidence.

The IFR trigger is used to identify decays to two $\mu^{+} \mu^{-}$and cosmic rays and will not be discussed here since these events are mostly used for background and luminosity studies. DCH and EMC subtriggers are produced and assembled within a fixed latency window of 11-12 $\mu$ s after the collision, and are delivered to the FCTS where the final decision is made. If necessary, FCTS inform the subsystems to send their information to the level 3 trigger. Data used to form the trigger decision are preserved with each event for efficiency studies.

The L1 is configured to have an output rate of about $2 \mathrm{kHz}$ at design luminosity. Out of this total rate, about $10 \%$ is produced from Bhabha and annihilation physics events, whereas cosmic ray and random beam crossing triggers contribute to another $10 \%$. The 
remaining triggers are due to lost particles interacting with the beam pipe or other components. The $z_{0}$ distribution of all accepted L1 track as reconstructed by L3 is shown in figure 3.16 and illustrates how a large fraction of the L1 tracks originate from backgrounds. Tracks produced near the IP $(z=0)$ correspond to physics events, whereas the peaks centered around $z= \pm 20 \mathrm{~cm}$ correspond to a flange joint on the beam pipe.

\subsubsection{L3 trigger}

The level 3 trigger is an online application that acts primarily as an event filter and is responsible for making a logging decision based on the output of the L1 hardware trigger. The L3 trigger is performed in three stages using 32 Unix processors operating in parallel mode. In the first stage, events are classified according to the DCH and EMC trigger information from the FCTS. In the second stage, algorithms are applied to the complete event data to provide a partial event reconstruction. Quantities of interest are then extracted, and filters are applied to test whether these quantities satisfy specific criteria. The last stage creates the L3 output, which identifies each event as either physics or flags the special categories of events needed for luminosity determination, diagnostics, and calibration purposes. 


\title{
Chapter 4
}

\section{Data and Monte Carlo Samples}

\author{
In data veritas
}

\subsection{On-peak and off-peak data}

The analysis presented in this dissertation is based on the 2000 to 2002 data. This corresponds to an integrated luminosity of $81.38 \mathrm{fb}^{-1}$ recorded at the $\Upsilon(4 S)$ resonance ("onpeak" data) or 88.35 million $B \bar{B}$ events. An additional integrated luminosity of $9.58 \mathrm{fb}^{-1}$ was recorded below the $\Upsilon(4 S)$ resonance ("off-peak" data). This allows the study of the underlying continuum events, $e^{+} e^{-} \rightarrow f \bar{f}$, where $f$ may be any of the charged leptons or lighter quarks $u, d, s$, or $c$. The breakdown of the integrated luminosity for each year is listed in table 4.1.

\subsection{Monte Carlo simulations}

In order to understand the performance of the detector and evaluate backgrounds, Monte Carlo simulations are produced by the BABAR Collaboration, typically in a ratio 3:1 with respect to on-peak data. The various Monte Carlo samples used in this work are presented in table 4.1. The production of the Monte Carlo is performed in three stages. First, physical processes are simulated by an event generator and particle four-vectors are recorded. The generator simulates the spread of energies allowed in the PEP-II beam collisions to determine the energy available to the resulting particles. The particles are then propagated 


\begin{tabular}{|l|c|c|}
\hline Data set & $N_{B \bar{B}} \times 10^{6}$ & $\mathcal{L}\left(\mathrm{fb}^{-1}\right)$ \\
\hline \hline 2000 Data on-resonance & 21.83 & 20.25 \\
2000 Data off-resonance & & 2.60 \\
2001 Data on-resonance & 39.17 & 35.59 \\
2001 Data off-resonance & & 3.79 \\
2002 Data on-resonance & 27.35 & 25.54 \\
2002 Data off-resonance & & 3.19 \\
\hline \hline Non-resonant signal MC & 2.46 & 523.4 \\
Resonant signal MC & 3.95 & 840.4 \\
$B^{0} \bar{B}^{0}$ MC & 109 & 207.6 \\
$B^{+} B^{-}$MC & 121 & 230.5 \\
\hline
\end{tabular}

Table 4.1: Integrated luminosity and number of $B \bar{B}$ events for data and Monte Carlo samples.

through a model of the material of the BABAR detector. Interactions between these particles and the material are simulated using the GEANT4 simulation package [47]. The energy, charge and angular information are used to calculate positions and idealized energy deposits in the detector, and the information is stored in a database. Note that while GEANT4 provides particle algorithms, the BABAR Collaboration developed the routines specific to the simulation of its detector response. Finally, the information from the database is retrieved and digitized, i.e. it is transformed into realistic signals which mimic those collected from the detector electronics. Real background events are stored in the database and are mixed with simulated events to more closely reproduce the data. Level 1 and level 3 triggers are then applied to the event. The end result is an event analogous to real data recorded by the BABAR detector.

\subsubsection{Simulation of signal events}

Two different $B \rightarrow X_{u} \ell \bar{\nu}_{\ell}$ signal Monte Carlo samples are used in this analysis. In the first sample, non-resonant $B \rightarrow X_{u} \ell \bar{\nu}_{\ell}$ events are simulated according to the triple differential decay width $d \Gamma_{u}^{\mathrm{SL}} / d m_{X} d E_{\ell} d q^{2}$ model defined in section 2.5.6, such that final state hadrons are produced with a continuous invariant mass spectrum. Subsequent hadronization of the 
$X_{u}$ system is handled by JETSET [48]. This generator has the advantage that it allows studies of the change in the fraction of signal events as input parameters are varied, but it cannot produce hadronic states with mass below $2 m_{\pi}$, and does not produce any resonant structure in the hadronic mass spectrum.

A resonant signal sample is therefore used in conjunction to the non-resonant sample. In this case, exclusive charmless semileptonic decays are generated using the ISGW2 model [23]. The branching fractions for the various resonant decay modes are listed in Table 4.2. These branching fractions were set to reflect the latest world average values [18] and to enforce isospin conservation which dictates relations amongst the decays to pseudo-scalar and vector final states, and requires that the $B^{+} / B^{0}$ branching fractions be in the same ratio as the $B^{+} / B^{0}$ lifetimes, namely 1.08 . The mass spectra of the $X_{u}$ particle produced by both generators are displayed in figure 4.1.

\begin{tabular}{|c|c|c|c|c|c|}
\hline \multirow{2}{*}{$\begin{array}{l}\frac{B^{0} \text { mode }}{} \\
B^{0} \rightarrow \pi^{-} \ell^{+} \nu\end{array}$} & \multicolumn{2}{|c|}{$\mathrm{BF} \times 10^{6}$} & $B^{+}$mode & \multicolumn{2}{|c|}{$\mathrm{BF} \times 10^{6}$} \\
\hline & $\overline{133}$ & 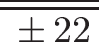 & 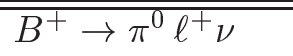 & 72 & 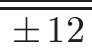 \\
\hline & & & $B^{+} \rightarrow \eta \ell^{+} \nu$ & 84 & \pm 36 \\
\hline$B^{0} \rightarrow \rho^{-} \ell^{+} \nu$ & 269 & $\begin{array}{l}+74 \\
-77\end{array}$ & $B^{+} \rightarrow \rho^{0} \ell^{+} \nu$ & 145 & $\begin{array}{l}+40 \\
-41\end{array}$ \\
\hline & & & $B^{+} \rightarrow \omega \ell^{+} \nu$ & 145 & $\begin{array}{l}+40 \\
-41\end{array}$ \\
\hline & & & $B^{+} \rightarrow \eta^{\prime} \ell^{+} \nu$ & 84 & \pm 84 \\
\hline Hybrid inclusive & 2271 & & Hybrid inclusive & 2357 & \\
\hline Total & 2673 & & Total & 2887 & \\
\hline
\end{tabular}

Table 4.2: Branching fractions used in the hybrid model for $B \rightarrow X_{u} e \bar{\nu}_{e}$ decays. The total branching fraction appearing in this table was determined in preliminary results.

Neither the non-resonant nor the exclusive generator provides a realistic simulation of $B \rightarrow X_{u} \ell \bar{\nu}_{\ell}$ decays, since the former lacks important resonant structure at low mass and the latter is incomplete and contains no non-resonant decays. Therefore, these two samples are mixed to provide a more realistic simulation. The resulting model, which will be referred to as the hybrid sample throughout this dissertation, is constructed as follows. First, the phase space in $m_{X}, q^{2}$ and $E_{\ell}$ is divided into a $8 \times 8 \times 8$ array. The segmentation of the phase-space is presented in table 4.3. The hybrid model is then a combination of the 

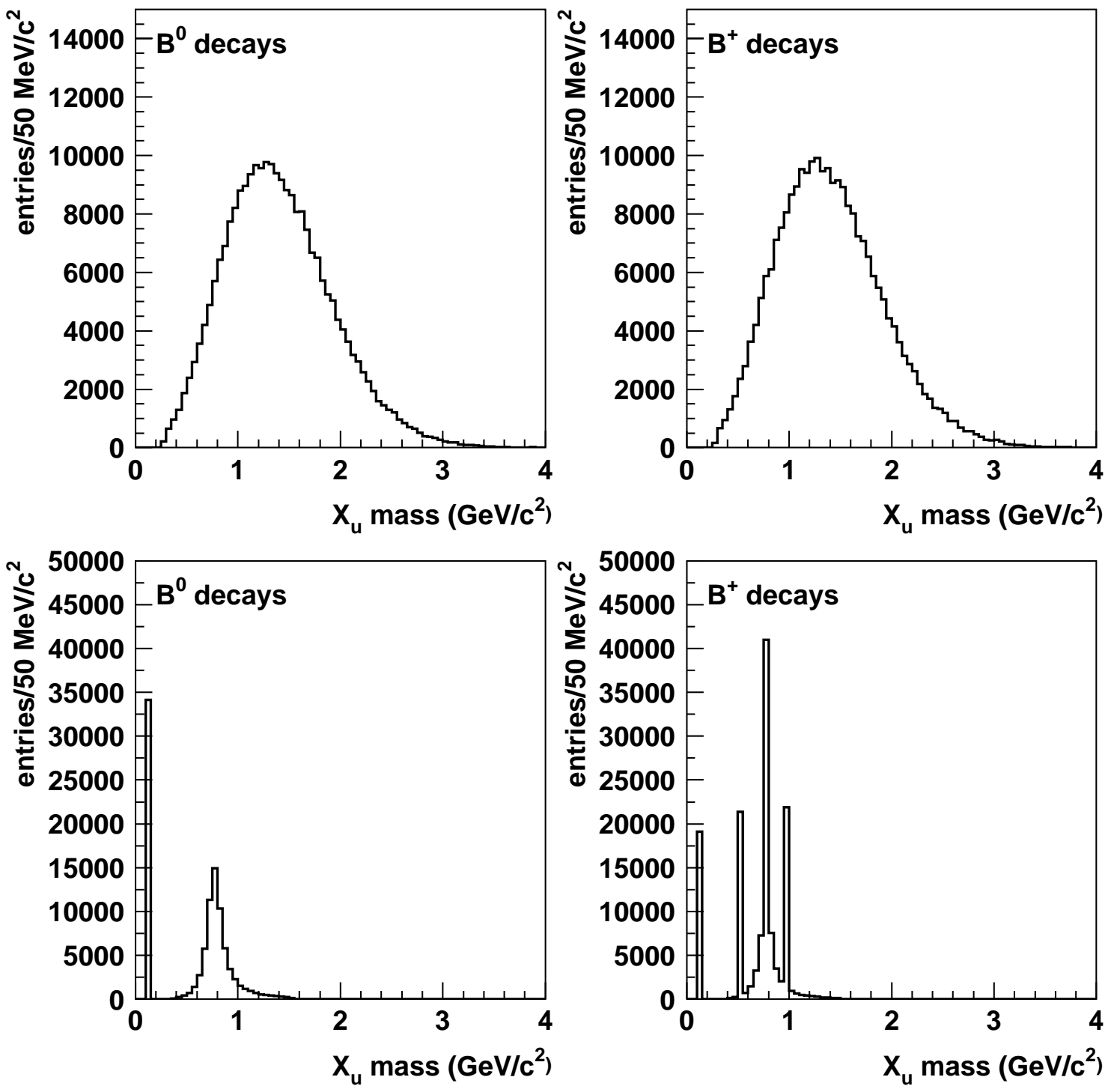

Figure 4.1: Mass spectra of the generated $X_{u}$ from $B^{0}$ and $B^{+}$decays using the non-resonant (top row) and resonant (bottom row) generators. 


\begin{tabular}{|lc|cccccccc|}
\hline \multicolumn{2}{|l|}{ Kinematic variable } & 1 & 2 & 3 & 4 & 5 & 6 & 7 & 8 \\
\hline \hline$m_{X}$ & $\left(\mathrm{GeV} / c^{2}\right)$ & 0.0 & 1.4 & 1.6 & 1.8 & 2.0 & 2.5 & 3.0 & 3.5 \\
\hline$q^{2}$ & $\left(\mathrm{GeV}^{2} / c^{4}\right)$ & 0.0 & 2.5 & 5.0 & 7.5 & 10.0 & 12.5 & 15.0 & 20.0 \\
\hline$E_{\ell}$ & $(\mathrm{GeV})$ & 0.00 & 0.50 & 1.00 & 1.25 & 1.50 & 1.75 & 2.00 & 2.25 \\
\hline
\end{tabular}

Table 4.3: Segmentation of the signal $B \rightarrow X_{u} e \bar{\nu}_{e}$ hybrid phase-space in $m_{X}, q^{2}$ and $E_{\ell}$. The values shown correspond to the lower edge of a given bin. Overall, the phase-space is divided into $8 \times 8 \times 8=512$ bins.

exclusive and inclusive models, such that for each bin $i$

$$
H_{i}=R_{i}+w_{i} N_{i},
$$

where $H_{i}, R_{i}$ and $N_{i}$ represent the bin content from hybrid, resonant and non-resonant samples, respectively. The determination of the weight $w_{i}$ is discussed below.

The overall normalization of the non-resonant component of the hybrid is first adjusted to give the proper total $B \rightarrow X_{u} \ell \bar{\nu}_{\ell}$ branching fraction (see table 4.2). The resonant and non-resonant samples are then mixed such that the fraction of hybrid events for a given bin $i, H_{i} / \sum_{j} H_{j}$, is the same as for the non-resonant sample, $N_{i} / \sum_{j} N_{j}$, resulting in a weight $w_{i}$ for each bin. If the $w_{i}$ obtained from this procedure is negative it is then set to $w_{i}=0$. Finally, a global normalization factor is applied to the weights to maintain the proper normalization. This is necessary to account for setting negative $w_{i}$ to zero. In practice, this factor is never larger than 1.00-1.02. The kinematic distributions of the hybrid model are shown and compared with those of the non-resonant sample in figures 4.2 to 4.5 . As expected, the hybrid $E_{e}$ and $q^{2}$ spectra match well the non-resonant model.

\subsubsection{HQE parameters}

Precise measurements of the HQE parameters $\bar{\Lambda}$ and $\lambda_{1}$ from moments of the electron momentum and hadronic mass spectra in $B \rightarrow X_{c} e \bar{\nu}_{e}$ decays [25] have recently become available. Unfortunately, these values cannot be used directly in the theoretical model used throughout the development of this analysis [49]. For the purpose of estimating signal efficiencies, the above non-resonant $B \rightarrow X_{u} \ell \bar{\nu}_{\ell}$ model of [38] is used and the shape function 

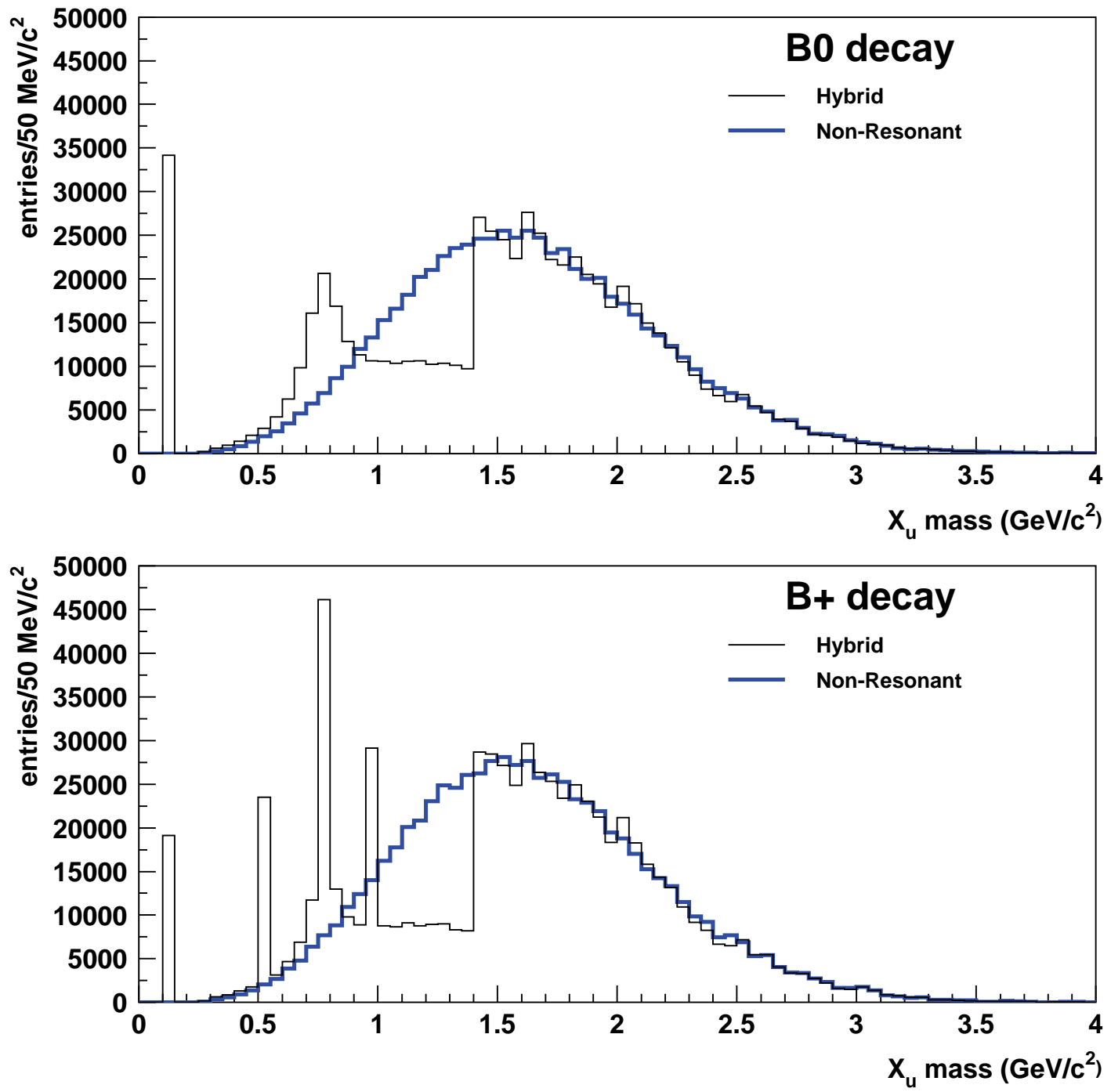

Figure 4.2: $\mathrm{MC} X_{u}$ mass spectrum for $B^{0}$ and $B^{+}$decays. The resulting hybrid signal sample can be compared with the ideal non-resonant model described in [38]. 

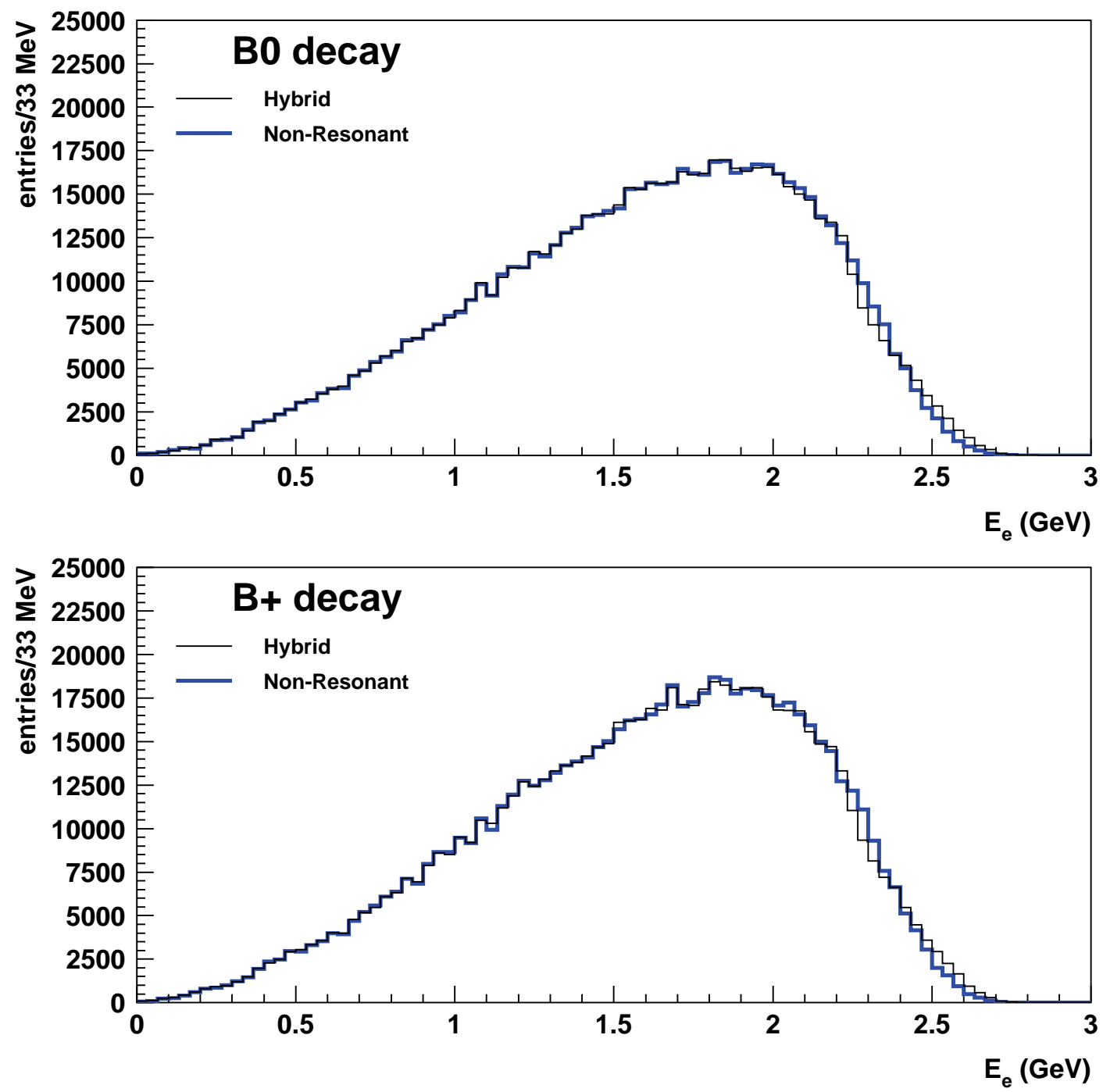

Figure 4.3: $\mathrm{MC}$ electron energy spectrum for $B^{0}$ and $B^{+}$decays. The resulting hybrid signal sample can be compared with the ideal non-resonant model described in [38]. 

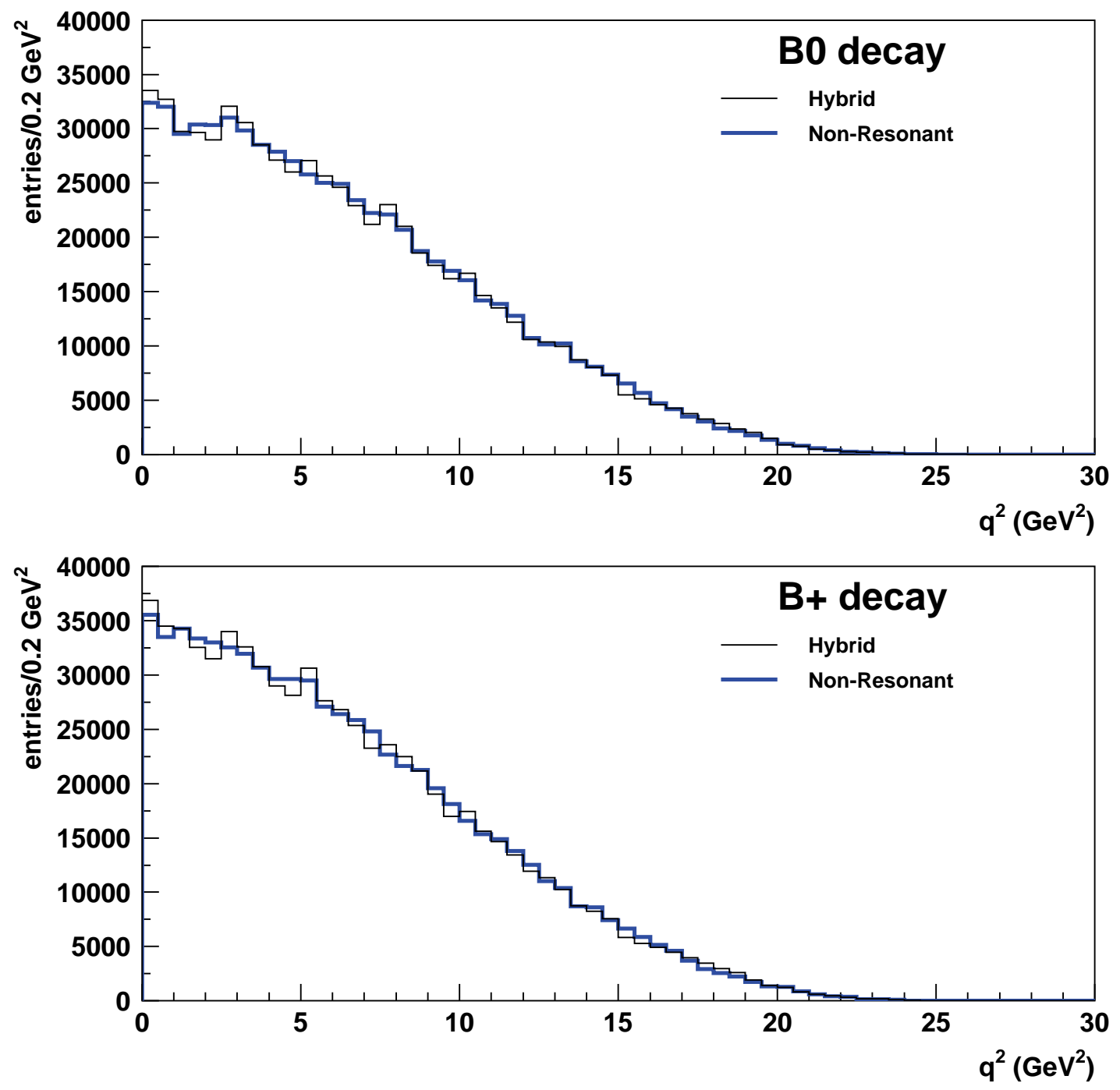

Figure 4.4: $\mathrm{MC} q^{2}$ spectrum for $B^{0}$ and $B^{+}$decays. The resulting hybrid signal sample can be compared with the ideal non-resonant model described in [38]. 

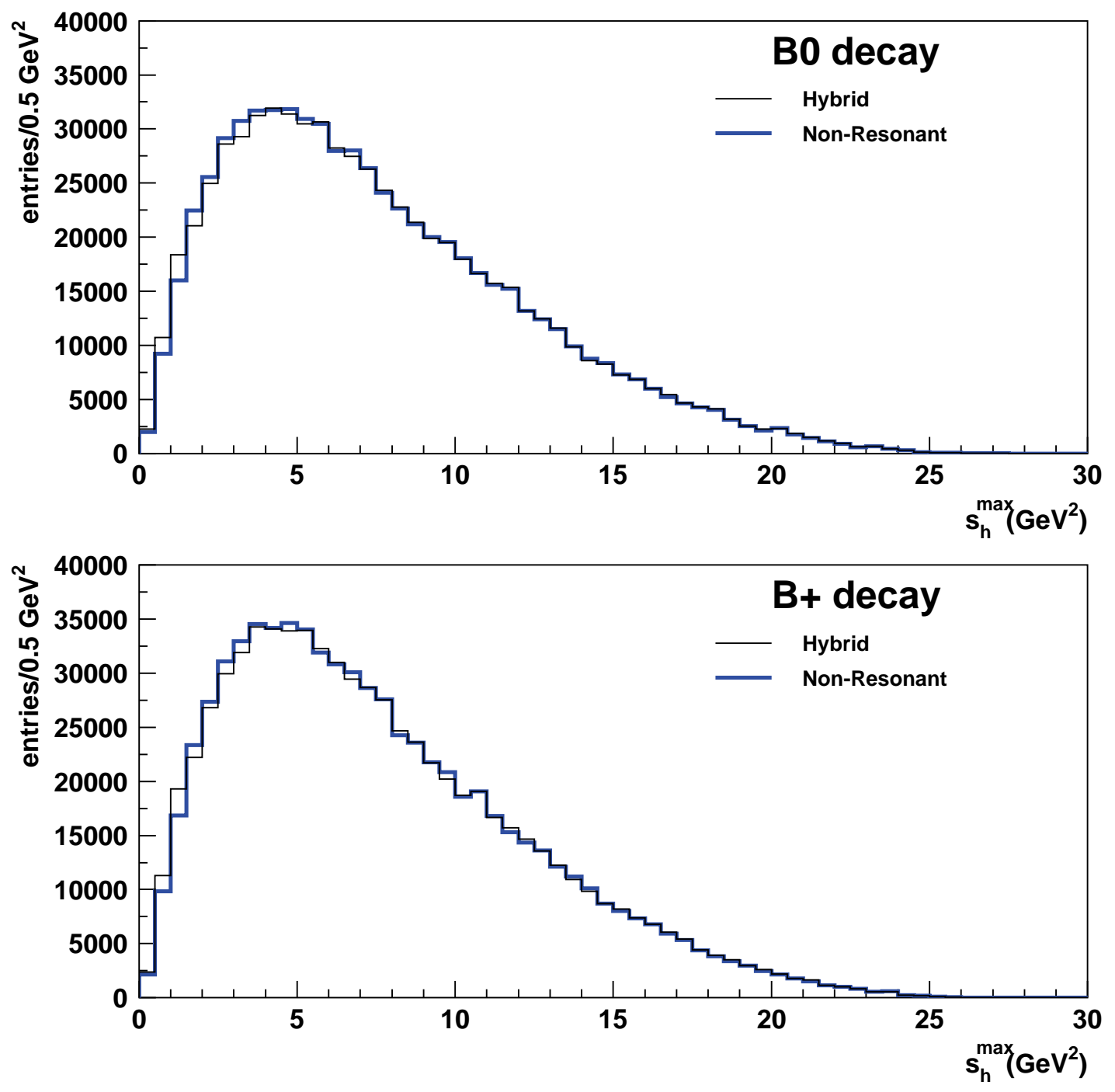

Figure 4.5: $\mathrm{MC} s_{\mathrm{h}}^{\max }$ spectrum $B^{0}$ and $B^{+}$decays. The resulting hybrid signal sample can be compared with the ideal non-resonant model described in [38]. 
(HQE) parameters are extracted from the measurements of the first and second energy moments in $B \rightarrow X_{s} \gamma$ which allows a constraint on the parameters $m_{b}$ and $a$. Such measurements were first performed by the CLEO collaboration [50]. The two-dimensional 1- $\sigma$ contour measured in this study can be described by a set of discrete points from which the following points were selected:

$$
\begin{aligned}
\left\{m_{b}, a\right\}= & \{4.845,2.55\},\{4.48,0.574\},\{4.785,1.15\},\{4.785,3.59\},\{4.735,0.896\}, \\
& \{4.69,0.68\},\{4.69,2.05\},\{4.58,0.55\},\{4.58,1.13\},\{4.53,0.56\}
\end{aligned}
$$

with the central value located at $\{4.735,1.6\} .{ }^{1}$ A more recent study of $B \rightarrow X_{s} \gamma$ has been performed and yields

$$
\begin{aligned}
\left\{m_{b}, a\right\}= & \{4.68,2.87\},\{4.64,3.89\},\{4.64,1.45\},\{4.62,2.27\},\{4.60,1.23\}, \\
& \{4.60,3.20\},\{4.57,2.44\},\{4.57,2.45\},\{4.53,1.53\}
\end{aligned}
$$

with the central point now located at $\{4.62,2.27\}$ [40]. From a general consensus within the BABAR Collaboration, the latter set of parameters are considered for this analysis [51].

\subsubsection{Simulation of backgrounds}

Monte Carlo samples were used to provide background estimates for $B^{0} \bar{B}^{0}$ and $B^{+} B^{-}$ events. The number of events generated and the equivalent integrated luminosity are listed in table 4.1. These backgrounds may be classified into two categories: charm and other backgrounds.

Charm backgrounds are produced in semileptonic $B \rightarrow X_{c} \ell \bar{\nu}_{\ell}$ decays, and will often be referred to as $b \rightarrow c$ events. The other backgrounds consist of events where the charged leptons are produced in secondary decays (cascades), or more rarely, are charged tracks that have been misidentified as leptons (fakes). These events will be referred to as non- $b \rightarrow c$ events.

The branching fraction for semileptonic $B$ decays to $D$ mesons were initially set to the values listed in table 4.4. If a particular $B^{0}$ or $B^{+}$branching fraction measurement was

\footnotetext{
${ }^{1}$ The $b$-quark mass is expressed in units of $\mathrm{GeV} / c^{2}$.
} 


\begin{tabular}{|l|c|c|}
\hline Decay type & $\mathcal{B}\left(B^{0}\right)$ & $\mathcal{B}\left(B^{+}\right)$ \\
\hline \hline$B \rightarrow D \ell \nu$ & $2.07 \pm 0.15$ & $2.24_{-0.16}^{+0.15}$ \\
$B \rightarrow D^{*} \ell \nu$ & $5.70_{-1.02}^{+0.53}$ & $6.17_{-1.13}^{+0.59}$ \\
$B \rightarrow D_{1} \ell \nu$ & $0.52 \pm 0.15$ & $0.56 \pm 0.16$ \\
$B \rightarrow D_{2}^{*} \ell \nu$ & $0.23 \pm 0.23$ & $0.30 \pm 0.30$ \\
$B \rightarrow D_{0}^{*} \ell \nu$ & $0.45_{-0.45}^{+0.41}$ & $0.49_{-0.49}^{+0.38}$ \\
$B \rightarrow D_{1}^{\prime} \ell \nu$ & $0.83_{-0.83}^{+0.41}$ & $0.90_{-0.90}^{+0.38}$ \\
\hline$B \rightarrow D \ell \nu X$ & $10.21 \pm 0.17$ & $11.04 \pm 0.18$ \\
\hline
\end{tabular}

Table 4.4: Branching fractions for semileptonic $B$ decays to $D$ meson. The inclusive rate is saturated with decays of the type $B \rightarrow D n(\pi) \ell \nu$. These branching fractions correspond to the latest world average values [18] and results reported in [52] and [53].

unavailable, the branching fraction was set based on isospin symmetry and the measured $B^{+} / B^{0}$ lifetime ratio. The total $B \rightarrow X_{c} \ell \bar{\nu}_{\ell}$ rate was finally obtained by adding decays of the type $B \rightarrow D n(\pi) e \bar{\nu}_{e}$ based on the model of Goity and Roberts [54]. This contribution amounts to $0.41 \%$ for $B^{0}$ and $0.38 \%$ for $B^{+}$. The above branching ratios were eventually adjusted to match the data more closely as will be discussed in section 6.2. 


\title{
Chapter 5
}

\section{Selection of Semileptonic Decays}

\author{
Labor omnia vincit improbus \\ -Virgil
}

\subsection{Analysis strategy}

In order to measure the inclusive $B \rightarrow X_{u} e \bar{\nu}_{e}$ branching fraction, data and Monte Carlo yields are extracted for the selected portion of the $B \rightarrow X_{u} e \bar{\nu}_{e}$ phase-space. The signal yield is computed as the number of candidates within the signal region in data, $N_{\text {cand }}^{\text {data }}$, minus the number of background events estimated from the Monte Carlo simulation, $N_{\mathrm{bkg}}$. The branching fraction is then measured as

$$
\mathcal{B}_{u}^{\mathrm{SL}}=\frac{N_{\mathrm{cand}}^{\text {data }}-N_{\mathrm{bkg}}}{\epsilon_{u} N_{B}}
$$

where $\epsilon_{u}$ is the $B \rightarrow X_{u} \ell \bar{\nu}_{\ell}$ efficiency evaluated using the signal simulation and $N_{B}$ is the total number of $B$ mesons within the data sample. Therefore, to reduce the above measurement sensitivity to uncertainties in the modeling of background decays, it is imperative to suppress backgrounds, in particular $B \rightarrow X_{c} \ell \bar{\nu}_{\ell}$ events, as much as possible.

In this analysis, semileptonic $B$ decays are first selected using the criteria outlined in section 5.2, which consist of an event shape selection and the presence of a high energy electron. The discrimination from charm backgrounds is based on the determination of the invariant mass squared of the electron-neutrino system. It is obtained by combining the measured electron momentum with an estimate of the neutrino inferred from a measurement 
of the missing momentum of the event.

Hence, the particle selection described in section 5.3 is designed to provide an excellent total energy and momentum reconstruction while eliminating regions where data and Monte Carlo simulations do not agree well. The adequacy of this selection is demonstrated in section 5.3.6 in terms of the improvements seen in the neutrino reconstruction.

Further criteria are applied to the events to guarantee a proper neutrino reconstruction and to optimize the signal to background ratio. The event selection must also define a region of the phase-space where the simulation follows closely the data. Section 5.5 will describe the optimization procedure and the criteria adopted for this analysis.

Since the evaluation of the background content in the signal extraction relies on the simulation, a $B \rightarrow D^{0} e \bar{\nu}_{e}$ control sample is used to reduce this dependence. This control sample may also be used to adjust the background normalization and to study the neutrino reconstruction as will be discussed in chapter 6 .

Once the agreement between data and Monte Carlo is satisfactory for both the signal and control samples, systematic uncertainties on the measurement of $\mathcal{B}_{u}^{\mathrm{SL}}$ will be evaluated (chapter 7). Note that in order to avoid possible biases, this analysis was originally performed blind: the event selection and the evaluation of the systematic uncertainties were performed using Monte Carlo simulation and off-peak data only. The extraction of the signal yield in data and comparisons between data and the simulation within the signal region were performed only in the final stage of the analysis. Preliminary studies based on blinded data may be obtained in [55], but no blinding has been applied to the data shown in this dissertation.

\subsubsection{Continuum subtraction}

In order to study the properties of underlying continuum events, $e^{+} e^{-} \rightarrow f \bar{f}$ events were also simulated. Unfortunately, studies have shown a rather poor agreement with continuum data. To circumvent this problem, the data presented in this analysis is shown after the continuum component has been removed. This is done by subtracting from the on-peak 
sample the off-peak data scaled to on-peak luminosity for any quantity $x_{i}$ studied in data,

$$
\begin{aligned}
x_{i}^{\text {data }} & =x_{i}^{\text {on }}-r \cdot x_{i}^{\text {off }} \\
& =x_{i}^{\text {on }}-\frac{\mathcal{L}_{\text {on }}}{\mathcal{L}_{\text {off }}}\left(\frac{E_{\text {off }}}{E_{\text {on }}}\right)^{2} x_{i}^{\text {off }},
\end{aligned}
$$

where $E_{\text {off }}$ and $E_{\text {on }}$ are the off-peak and on-peak center of mass energies, respectively, and $r \approx 8.41$. In order to reduce the statistical uncertainties brought about by subtracting the scaled off-peak data, the selection described in section 5.5 includes criteria designed to suppress continuum events. Furthermore, the momenta of particles from off-peak data are scaled to account for the difference between off-peak and on-peak center of mass energies according to

$$
\begin{aligned}
& E_{\text {scaled }}=E_{\text {measured }} \frac{10.58 \mathrm{GeV}}{\sqrt{s}} \\
& \vec{p}_{\text {scaled }}=\vec{p}_{\text {measured }} \frac{\sqrt{E_{\text {scaled }}^{2}-m^{2}}}{\left|\vec{p}_{\text {measured }}\right|}
\end{aligned}
$$

where $m$ is the mass hypothesis assigned to the particle. Unless specified otherwise, this procedure will be used to obtain the results presented throughout this dissertation.

\subsection{Event preselection}

Events enter the analysis if they survive the requirements described in this section. Each event must satisfy the L3 trigger and the background filter criteria: $N_{\text {tracks }} \geq 3$ and $R_{2}<$ 0.98 , where $R_{2}$ is the ratio of the second to first Fox-Wolfram moments defined in [56]. The latter is a powerful method for discriminating $B \bar{B}$ events from lepton-pair backgrounds. In a true signal event, the primary $e^{+} e^{-}$pair produces a $B \bar{B}$ pair via the $\Upsilon(4 S)$ resonance. In the $\Upsilon(4 S)$ rest frame, the $B$ mesons have low momenta, such that the decay of each $B$ meson is nearly isotropic. As such, there are negligible correlations between the decay products coming from each of the two $B$ mesons.

However, in light-quark $(u, d, s)$ continuum events, the event shape has a pronounced two-jet structure, so there is a strongly preferred direction characterizing the whole 
event. These events therefore tend to have less isotropic decay shapes in the $\Upsilon(4 S)$ rest frame. In a $c \bar{c}$ event, the jet structure is still present, but is less pronounced, and event shape variables provide less discriminating power for this type of background.

Since this analysis is based on semileptonic decays, the event must also contain an identified electron with energy $E_{e}^{*}>1.4 \mathrm{GeV}$ in the center of mass frame to be retained. ${ }^{1}$ Because of the larger uncertainties associated with muon reconstruction, only semileptonic $B$ decays to electrons are considered. In addition, the event must survive a Bhabha veto, developed in [57], that requires the event to have a multiplicity greater than 5 objects

$$
N_{\text {tracks }}^{\prime}+N_{\gamma}^{\prime} / 2>5
$$

where $N_{\text {track }}^{\prime}$ is the number of tracks not being part of identified $\gamma \rightarrow e^{+} e^{-}$pairs, and $N_{\gamma}^{\prime}$ is the sum of photons with energy $>80 \mathrm{MeV}$ and identified $\gamma \rightarrow e^{+} e^{-}$pairs.

\subsection{Particle selection}

\subsubsection{General philosophy}

In order to reconstruct $q^{2}$, this analysis depends on the total event momentum and energy measurements. Hence, a particular approach to charged particles (tracks) and neutral particles (neutrals) selection is required. Criteria are applied to particles to discriminate between "primary particles", ${ }^{2}$ and particles produced by interactions with the detector material and artefacts from the reconstruction algorithms. This selection must also maintain a high efficiency and acceptance for primary particles while eliminating regions that are poorly simulated. Finally, the selection aims at improving the missing 4-momentum reconstruction. Clearly, the selection criteria chosen are a compromise to fulfill all of these requirements.

\footnotetext{
${ }^{1}$ The star, ${ }^{*}$, denotes quantities measured in the $\Upsilon(4 S)$ frame. This notation will be used throughout this dissertation.

${ }^{2}$ Particles produced from $e^{+} e^{-}$interactions.
} 


\subsubsection{Charged track selection}

Charged tracks are reconstructed in the drift chamber and/or silicon vertex tracker with parameters determined using a $\pi^{ \pm}$mass hypothesis. Selection criteria were obtained by comparing qualitatively data and Monte Carlo distributions. Regions where the agreement between generated and reconstructed MC quantities was poor, and regions with large discrepancies between data and $\mathrm{MC}$ simulation were removed. The charged track selection procedure is detailed in [58] and is summarized below. Requirements are made for:

1. The angle of the track in the lab frame, $0.41<\theta_{\text {lab }}<2.54$ (radians);

2. The momentum of the track in the lab frame, $p_{\text {lab }}<10 \mathrm{GeV} ;^{3}$

3. The transverse momentum of the track,

3a. For tracks with DCH hits, $p_{\mathrm{t}, \mathrm{lab}}>0.06 \mathrm{GeV}$;

3b. For tracks with SVT hits only, $0.06 \mathrm{GeV}<p_{\mathrm{t}, \mathrm{lab}}<0.2 \mathrm{GeV}$;

4. The distance of closest approach of the track, $|D O C A|<1.5 \mathrm{~cm}$;

5. The z-position of the track at the point of closest approach, $\left|z_{0}\right|<5 \mathrm{~cm}$;

6. Loopers and ghost candidates are vetoed using cuts specified below.

Loopers are produced by low momentum tracks trapped by the solenoidal magnetic field and looping in the tracking devices. As a result they are reconstructed as two or more tracks, depending on the momentum of the charged particle. Loopers are identified when a set of tracks satisfies the following:

1. The tranverse momentum is $p_{\mathrm{t}, \mathrm{lab}}<0.25 \mathrm{GeV}$ for each track;

2. Close angular separation between pairs of tracks corresponding to

\footnotetext{
${ }^{3}$ To simplify the notation, $c$ was set to 1 throughout this dissertation such that $\mathrm{GeV} / c \equiv \mathrm{GeV}$.
} 
2a. $\left|\Delta \phi_{\text {lab }}\right|<0.18,\left|\pi-\theta_{1, \text { lab }}-\theta_{2, \text { lab }}\right|<0.16$ if the charges associated with the pair of tracks are opposite;

2b. $\left|\Delta \phi_{\text {lab }}\right|<0.20,\left|\Delta \theta_{\text {lab }}\right|<0.18$ if the charges associated with the pair of tracks are equal;

3. Similar tranverse momenta, $\left|\Delta p_{\mathrm{t}, \mathrm{lab}}\right|<0.12 \mathrm{GeV}$.

For such a set of tracks, only the one produced closest to the interaction point is kept, i.e. the track with the smallest $\left|z_{0}\right|$, whereas the remaining tracks are marked as loopers and are not used further in the analysis.

Ghosts are artefacts from the tracking algorithms: multiple tracks are reconstructed from a set of DCH hits left by only one charged particle. Such tracks are identified as pairs of tracks for which:

1. $p_{\mathrm{t}, \mathrm{lab}}<0.35 \mathrm{GeV}$;

2. $\left|\Delta \phi_{\text {lab }}\right|<0.3$

3. $\left|\Delta \theta_{\text {lab }}\right|<0.3$

4. $\left|\Delta p_{\mathrm{t}, \mathrm{lab}}\right|<0.15 \mathrm{GeV}$;

5. $N_{\text {hits }}^{\mathrm{DCH}}($ track i $)+N_{\text {hits }}^{\mathrm{DCH}}($ track j $)<45$.

In this case, only the track with the greatest number of DCH hits is used in the analysis.

As can be seen in figure 5.1, the above selection improves the overall agreement between the data and simulation in terms of the charged track multiplicity and total energy reconstruction from the tracking system. Note that in this figure and all similar figures, the top plot features the data after continuum subtraction and the contribution from signal and background Monte Carlo simulations, and the bottom plot displays the comparison between data and complete (signal + background) simulation as $y_{i}^{\text {data }} / y_{i}^{\mathrm{MC}}-1$, where $y_{i}$ denotes the number of entries for a given bin $i$. Also provided in the lower plot is the $\chi^{2}$,

$$
\chi^{2}=\sum_{i} \frac{\left(y_{i}^{\mathrm{MC}}-y_{i}^{\mathrm{data}}\right)^{2}}{\sigma_{i}^{2}(\mathrm{MC})+\sigma_{i}^{2}(\text { data })},
$$


based purely on statistical uncertainties, and the number of bins (d.o.f.) used. The dominant systematic uncertainties will be discussed later in chapter 7. In order to enter the $\chi^{2}$ computation, a bin $i$ had to satisfy $y_{i}^{\mathrm{MC}}-3 \sigma_{i}(\mathrm{MC})>0$. Clearly, the $\chi^{2}$ improves after applying the charged track selection.

\subsubsection{Neutral energy selection}

All EMC bumps not matched to any charged track are considered suitable to be clusters and are contained in a neutral cluster list. The criteria for neutral energy (photons) selection were determined using the same philosophy used for the charged track selection and a detailed study may be found in [58]. Selection criteria are applied for:

1. The number of crystals in the cluster, $N_{\text {crystal }}>2$;

2. The energy of the cluster in the lab frame, $E_{\text {cluster }}>50 \mathrm{MeV}$;

3. The cluster lateral moment, LAT $<0.6$;

4. The angle $\theta$ of the cluster in the lab frame, $0.32<\theta_{\text {cluster }}<2.44$ (radians);

5. The angle between the positions of the cluster and the impact point of the nearest charged track at the EMC surface for tracks that do not satisfy the charged track selection,

$$
\Delta \alpha=\cos ^{-1}\left[\cos \theta_{\text {clus }} \cos \theta_{\text {trk }}+\sin \theta_{\text {clus }} \sin \theta_{\text {trk }} \cos \left(\phi_{\text {clus }}-\phi_{\text {trk }}\right)\right]<0.08 .^{4}
$$

Note that all of the above kinematic variables are measured in the laboratory frame. The lateral shower shape, LAT, was first used by the ARGUS Collaboration and is described in detail elsewhere [59]. Neutral objects not satisfying the above selection were excluded from the analysis.

The agreement between data and the simulation improves for both the neutral multiplicity and the total energy reconstructed, as can be seen in figure 5.2. The $\chi^{2} /$ d.o.f.

\footnotetext{
${ }^{4}$ No cut is made on this variable if the closest track is identified as an electron to enable bremsstrahlung photons to be retained.
} 

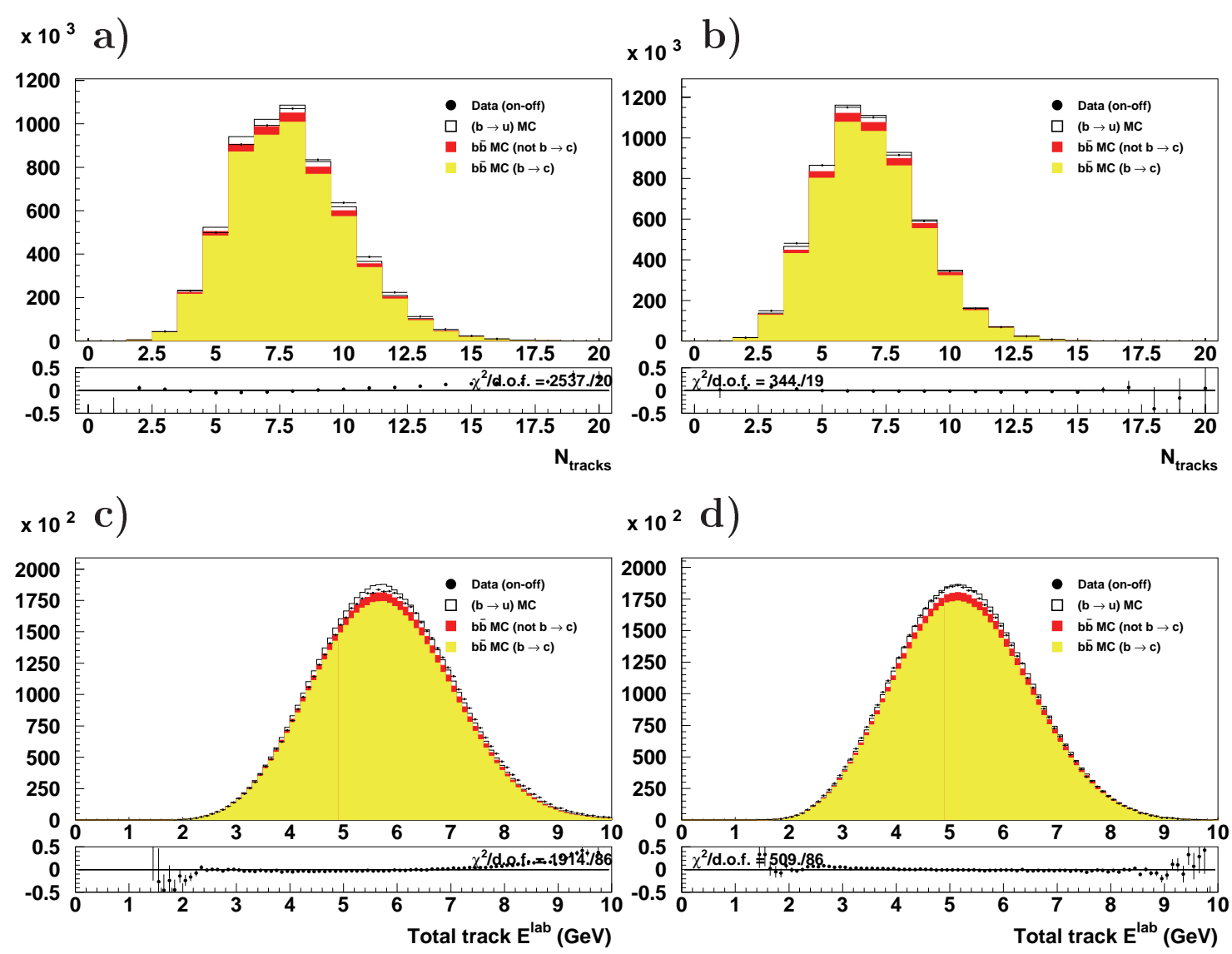

Figure 5.1: Distributions for the charged track multiplicity a) before and b) after applying the track selection; and the total energy reconstructed from the charged tracks c) before and after applying the selection criteria. The upper plots show the distributions from data after off-peak continuum subtraction as well as the Monte Carlo. Non- $b \rightarrow c$ events are either secondary electrons from charm decays or hadrons misidentified as electrons (see section 4.2.3). The lower plots show the relative comparison between data and MC as $y_{i}^{\mathrm{data}} / y_{i}^{\mathrm{MC}}-1$. The simulation is scaled to the number of $B \bar{B}$ pairs in the data. The events plotted satisfy the preselection described in section 5.2. 

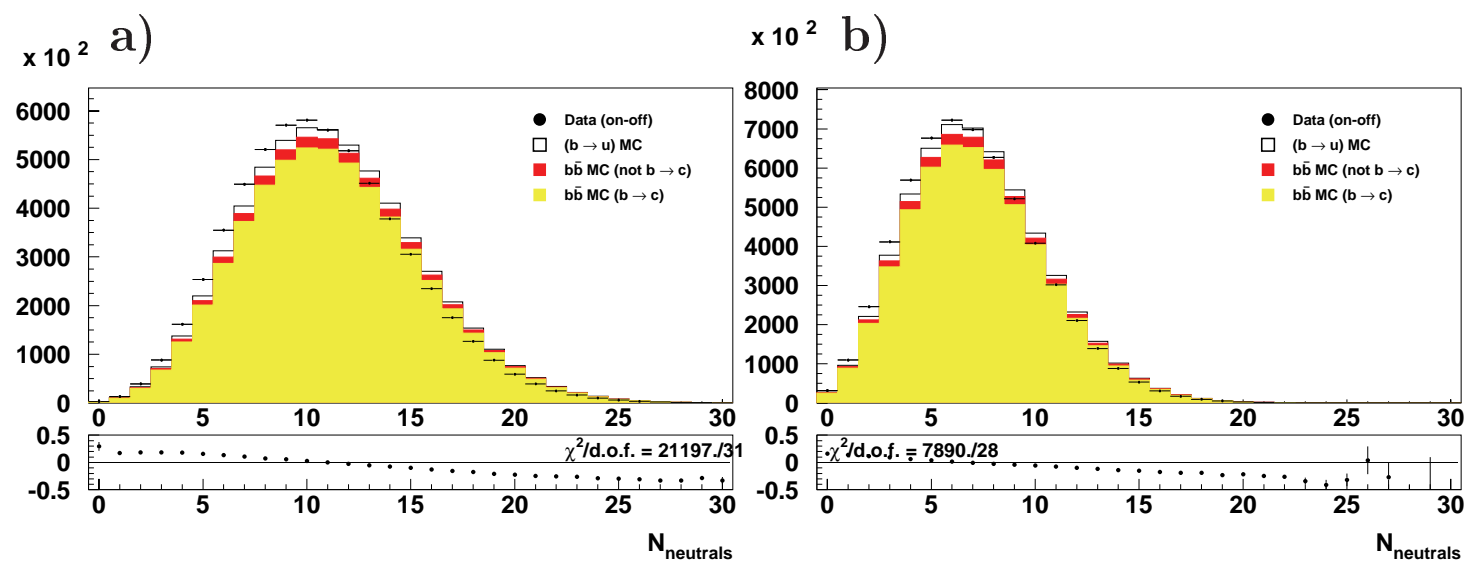

c)

d)
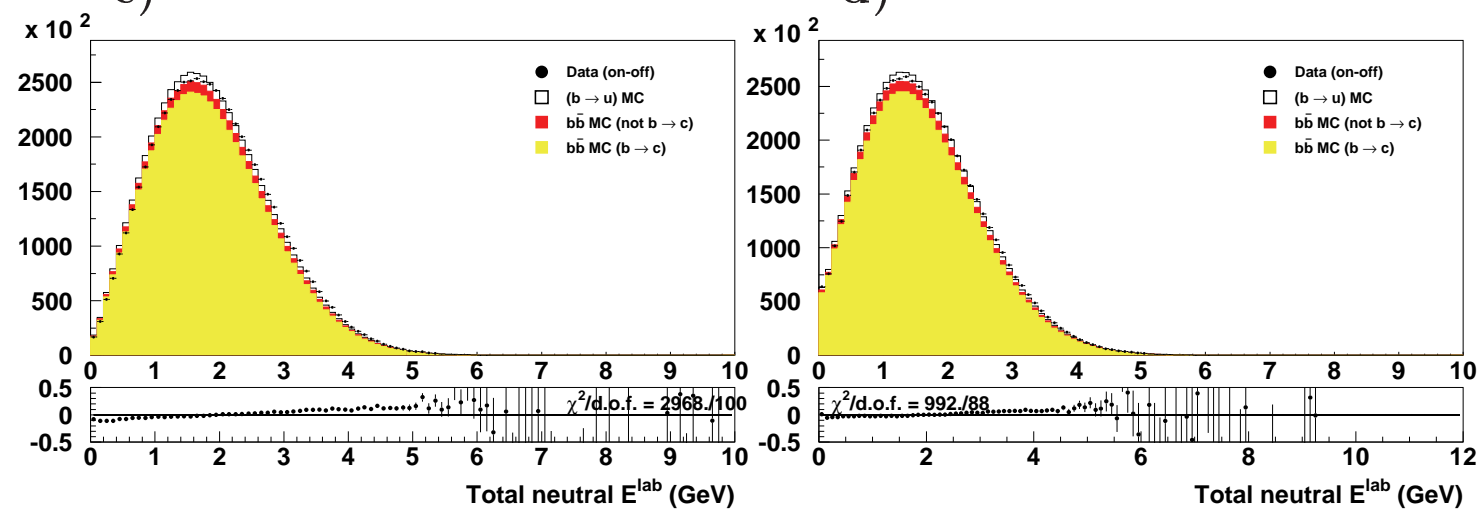

Figure 5.2: Distributions for the neutral deposits multiplicity a) before and b) after applying the neutral selection; and the total energy reconstructed from these EMC energy deposits c) before and d) after the applying the selection criteria. These plots follow the same conventions as those of figure 5.1.

is reduced by about a factor 3 in terms of the multiplicity and total energy reconstructed in the EMC.

\subsubsection{Composite particles}

Standard BABAR tools are used to reconstruct the following composite particles: $K_{S}^{0} \rightarrow$ $\pi^{+} \pi^{-}, \Lambda \rightarrow p \pi^{-}$and $\gamma \rightarrow e^{+} e^{-}[60]$. The $K_{S}^{0}$ selection requires a decay length $>2 \mathrm{~mm}$ and an invariant mass satisfying $0.4906 \mathrm{GeV}<m_{\pi \pi}<0.5047 \mathrm{GeV}$. The $\Lambda$ selection requires a decay length $>5 \mathrm{~mm}$ and a mass satisfying $1.112 \mathrm{GeV}<m_{p \pi^{-}}<1.120 \mathrm{GeV}$. 
Conversion photons must satisfy $m_{e e}<0.030 \mathrm{GeV}$. The standard $J / \psi$ sequence is also used to reconstruct $J / \psi \rightarrow \ell^{+} \ell^{-}$decays. Note that particles that were excluded by the charged track cuts when forming composites.

The set of composite particles produced by these selectors is then examined. If two or more composite candidates are found to share a common daughter track(s), then these composites are rejected. Similarly, if the association between a daughter track making a composite particle to a track in the charged track list was lost ${ }^{5}$ the composite candidate is rejected. All charged track daughters from the final set of accepted composite particles are removed from the charged track list.

The lepton daughters of reconstructed $J / \psi$ decays are not considered as leptons for the remainder of the analysis, neither as the signal electron nor as additional leptons, on which a veto will be placed.

\subsubsection{Particle identification}

Electrons are identified using a selector described in reference [61]. The need to reconstruct the total visible energy in this analysis also requires a best mass hypothesis to be defined for each accepted charged track. This is done by the following algorithm, where the first positive response determines the particle hypothesis to be used:

- Does the track satisfy the electron selection [61] ?

- Does the track satisfy the kaon selection [62]?

- Does the track satisfy the muon selection [63] ?

- Does the track satisfy the proton selection?

If all responses are negative, the particle is assumed to be a pion. A brief overview of particle identification is made in appendix ??.

\footnotetext{
${ }^{5}$ This is the case for electron daughters of $J / \psi$ on which bremsstrahlung recovery was performed.
} 


\subsubsection{Validation from missing momentum reconstruction}

The missing energy and momentum resolutions obtained after applying the particle selections defined in the previous sections are compared to the resolutions obtained prior to these selections. Note that the figures appearing in this section contain all data surviving the preselection criteria. Figure 5.3 shows the missing energy, $E_{\text {miss }}^{*}$, and missing momentum, $\left|\vec{P}_{\text {miss }}^{*}\right|$, distributions in the $\Upsilon(4 S)$ frame for both data and Monte Carlo simulation. A net improvement in the agreement between data and the simulation is observed after applying the refined particle selection. The $E_{\text {miss }}^{*}$ distribution is more affected by the selection requirements. This is not surprising as $\left|\vec{P}_{\text {miss }}^{*}\right|$ is less sensitive than $E_{\text {miss }}^{*}$ to mis-reconstructed particles since it involves the vector sum of the true missing momentum vector and the momentum vectors of additional missing particles, in which some cancellations occur that do not happen in the scalar sum $\left(E_{\text {miss }}^{*}\right)$. The energy component of the reconstructed neutrino 4-vector is therefore replaced with the magnitude of the missing momentum in calculating $q^{2}$, as will be discussed in section 5.4. Also shown in figure 5.3 is the quantity $E_{\text {miss }}^{*}-\left|\vec{P}_{\text {miss }}^{*}\right|$, an indicator of the quality of the neutrino reconstruction; since neutrinos are (nearly) massless, $E_{\text {miss }}^{*}-\left|\vec{P}_{\text {miss }}^{*}\right|$ should be zero within detector resolution. Missing particles, such as additional neutrinos, $K_{L}^{0}$ or particles lost due to detector acceptance, cause a positive bias in $E_{\text {miss }}^{*}-\left|\vec{P}_{\text {miss }}^{*}\right|$. Hence, negative $E_{\text {miss }}^{*}-\left|\vec{P}_{\text {miss }}^{*}\right|$ values can only come from mis-reconstruction. The particle selection clearly improves the agreement between the data and Monte Carlo simulation.

In figure 5.4, the signal Monte Carlo resolutions for the missing energy, $E_{\text {miss }}^{*}$ $E_{\nu, t r u e}^{*}$, and the missing momentum, $\left|\vec{P}_{\text {miss }}^{*}\right|-\left|\vec{P}_{\nu, \text { true }}^{*}\right|$, are shown prior to and after applying the particle selection criteria. Since the new selection has more stringent requirements, the mean of the $E_{\text {miss }}^{*}$ distribution is shifted to the positive side by $\sim 600 \mathrm{MeV}$, but the r.m.s. of the distribution remains about the same. The effect is less noticeable for $\left|\vec{P}_{\text {miss }}^{*}\right|$ and only a small $\simeq 24 \mathrm{MeV}$ bias is introduced while the r.m.s. stays the same within a few $\mathrm{MeV}$.

\footnotetext{
${ }^{6}$ Throughout this dissertation, the subscript $\nu$, true refers to the generated neutrino quantity.
} 


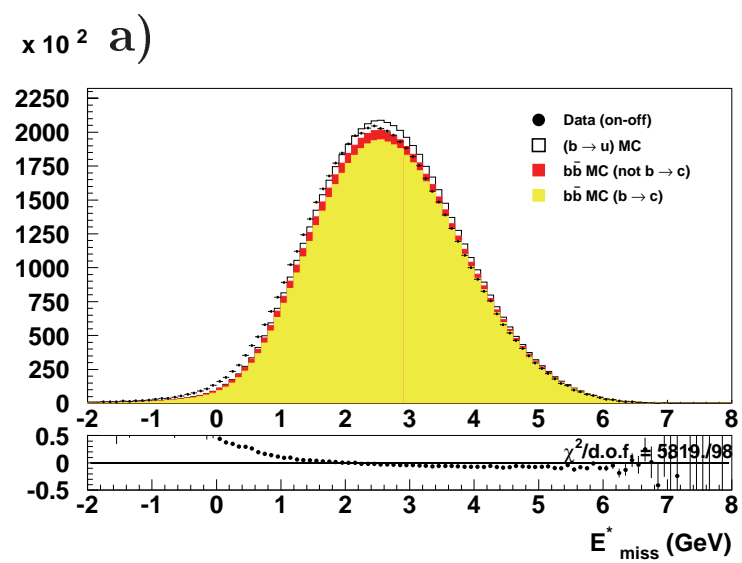

b)
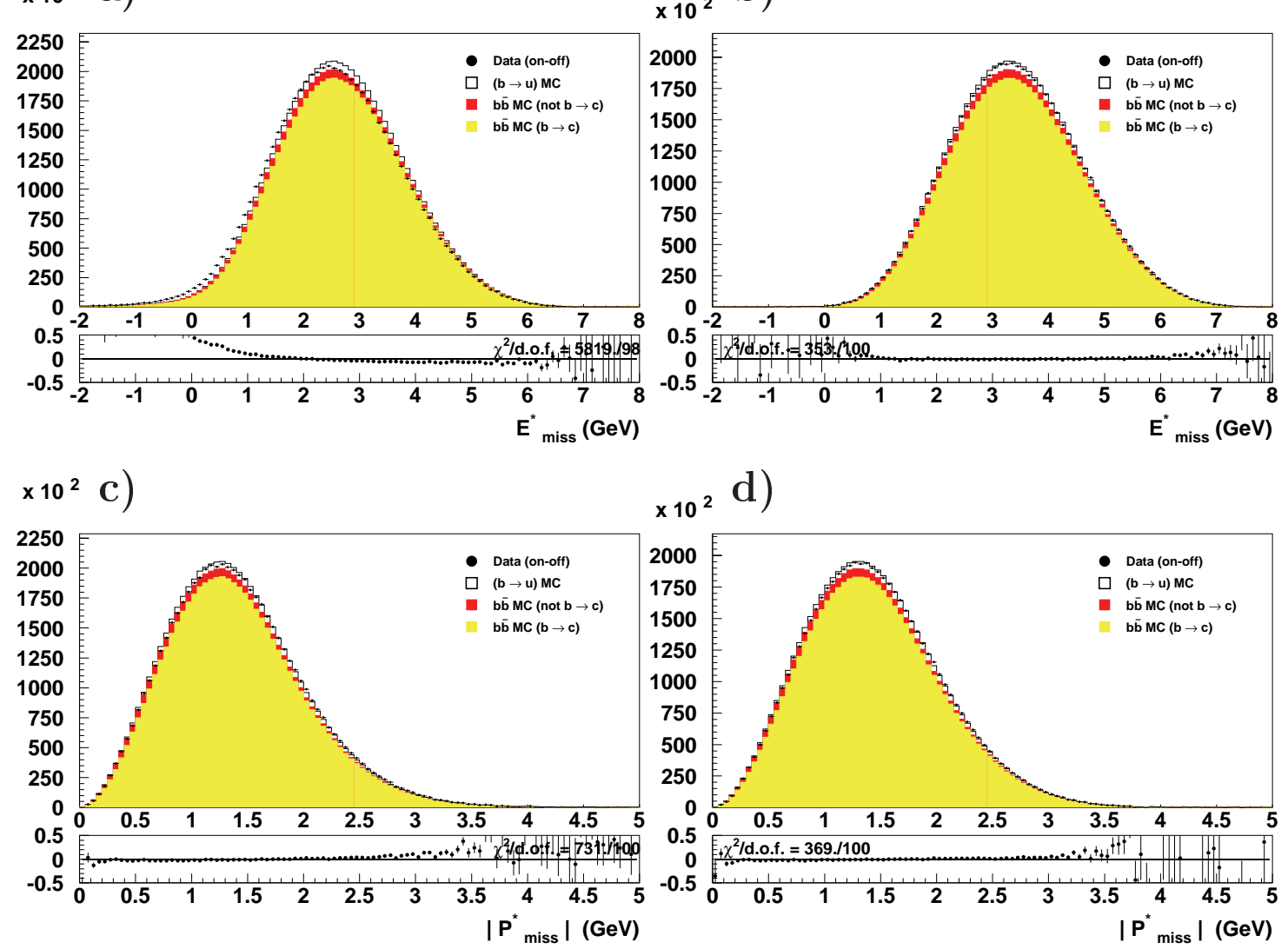

d)
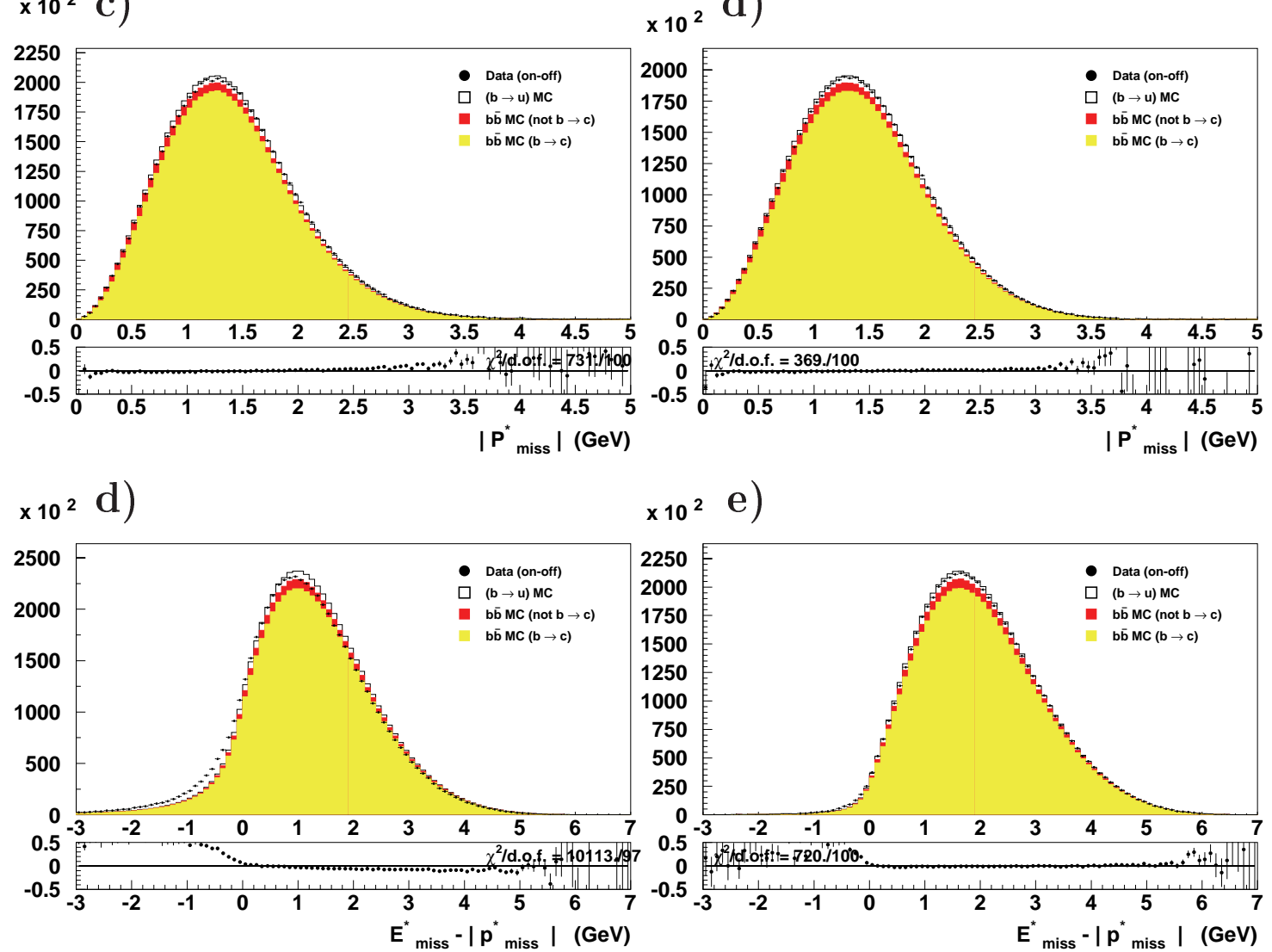

Figure 5.3: Data and Monte Carlo kinematical distributions prior to (left) and after (right) applying the particle selection described in sections 5.3.2 and 5.3.3. Displayed are distributions for the missing energy $E_{\text {miss }}^{*}$ (top), missing momentum $\left|\vec{P}_{\text {miss }}^{*}\right|$ (center) and the difference $E_{\text {miss }}^{*}-\left|\vec{P}_{\text {miss }}^{*}\right|$ (bottom). Note that the above quantities were computed in the $\Upsilon(4 S)$ frame. The plots follow the same conventions as those of figure 5.1. 
a)

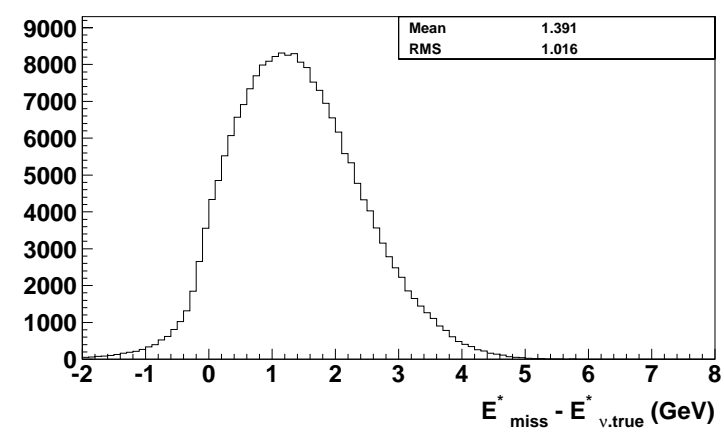

c)

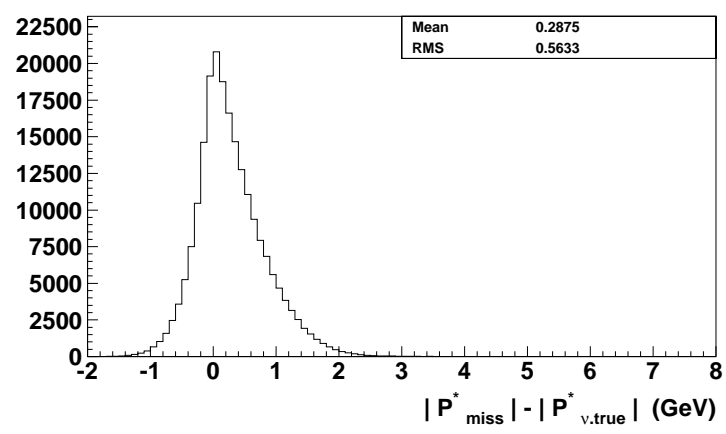

b)

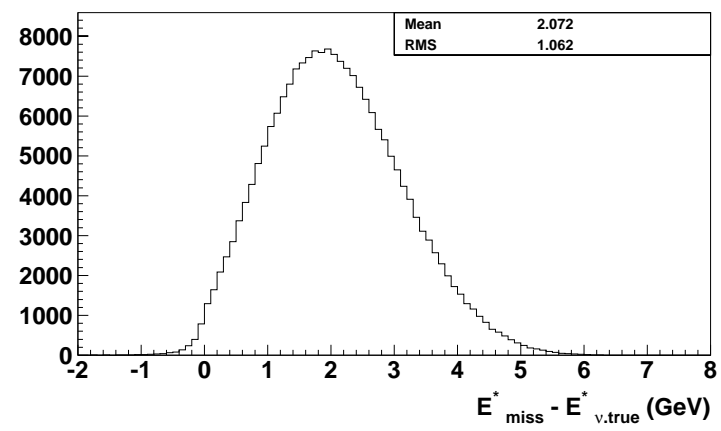

d)

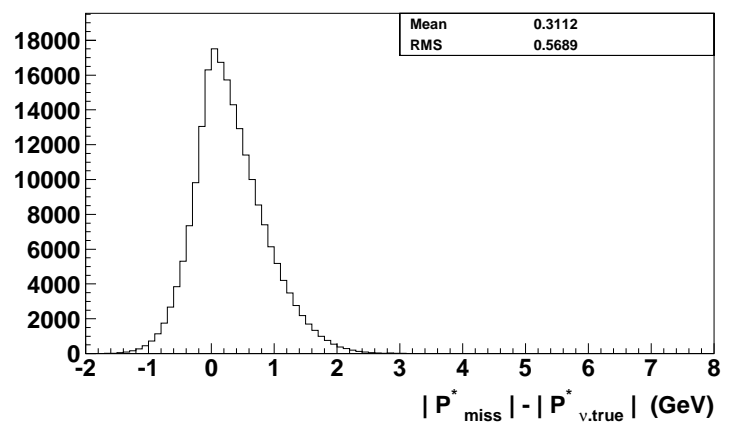

Figure 5.4: Signal Monte Carlo resolutions for the missing energy (top) and missing momentum (bottom) prior to (left) and after (right) applying the particle selection criteria. The quantities were computed in the $\Upsilon(4 S)$ frame.

Hence, the revised track and neutral selection improves the agreement between data and Monte Carlo and does not degrade the missing momentum resolution.

\subsection{Neutrino and $q^{2}$ reconstruction}

The electron energy, $E_{e}^{*}$, is directly measured by the detector whereas the neutrino momentum and $q^{2}$ need to be reconstructed from all of the decay products of the $\Upsilon(4 S)$. The procedure used to measure the neutrino momentum and $q^{2}$ is as follows:

- The visible 4-momentum, $p_{v i s}^{*}$, is computed by summing the 4-momenta from all accepted charged tracks, neutral EMC bumps and composite particles passing the 
selection described in 5.3;

- The $\Upsilon(4 S) 4$-momentum, $p_{\Upsilon(4 S)}^{*}$, has a zero 3-momentum and an energy equal to the $\sqrt{s}$ of the $e^{+} e^{-}$initial state;

- The missing 4-momentum is then computed as $p_{\mathrm{miss}}^{*}=p_{\Upsilon(4 S)}^{*}-p_{v i s}^{*}$;

- The magnitude of the missing momentum is used in place of the missing energy when calculating an estimator for the neutrino 4-vector in order to improve the neutrino energy resolution;

- The expected bias in $\left|\vec{P}_{\nu, \text { true }}^{*}\right|-\left|\vec{P}_{\text {miss }}^{*}\right|$ is corrected as a function of $\left|\vec{P}_{\text {miss }}^{*}\right|$ to derive an estimate of the neutrino momentum (discussed below);

- Finally, $q^{2}$ is calculated by computing the electron-neutrino invariant $q^{2}=\left(p_{e}^{*}+p_{\nu}^{*}\right)^{2}$.

Hence, the "observed" missing momentum in the detector is used to calculate the momentum of the "unseen" neutrino.

Based on the fact that neutrinos are nearly massless, one can remove some events where the neutrino momentum is not well determined by requiring the missing energy and the missing momentum to have similar magnitudes; accordingly, constraints are applied on $E_{\text {miss }}^{*}-\left|\vec{P}_{\text {miss }}^{*}\right|$. Note that the selection is not based on the missing invariant mass squared, $m_{\text {miss }}^{2}$, because it involves both physics $\left(E_{\text {miss }}^{*}+\left|\vec{P}_{\text {miss }}^{*}\right|\right)$ and resolution $\left(E_{\text {miss }}^{*}-\left|\vec{P}_{\text {miss }}^{*}\right|\right)$. If the direction of the missing momentum vector points toward uninstrumented regions of the detector (i.e. near the beamline) one cannot distinguish between neutrinos and inherently detectable particles that are outside the acceptance; as a result, events for which $\left|\cos \theta_{\text {miss }}^{*}\right| \sim 1$ are removed. The requirements made on these variables are part of the signal optimization described in section 5.5.

The missing momentum vector is a biased estimator of the true neutrino momentum. Studies performed on Monte Carlo simulations show that this bias arises principally from the presence of $K_{L}^{0}$ or additional neutrinos in an event, each of which account for about $45 \%$ 

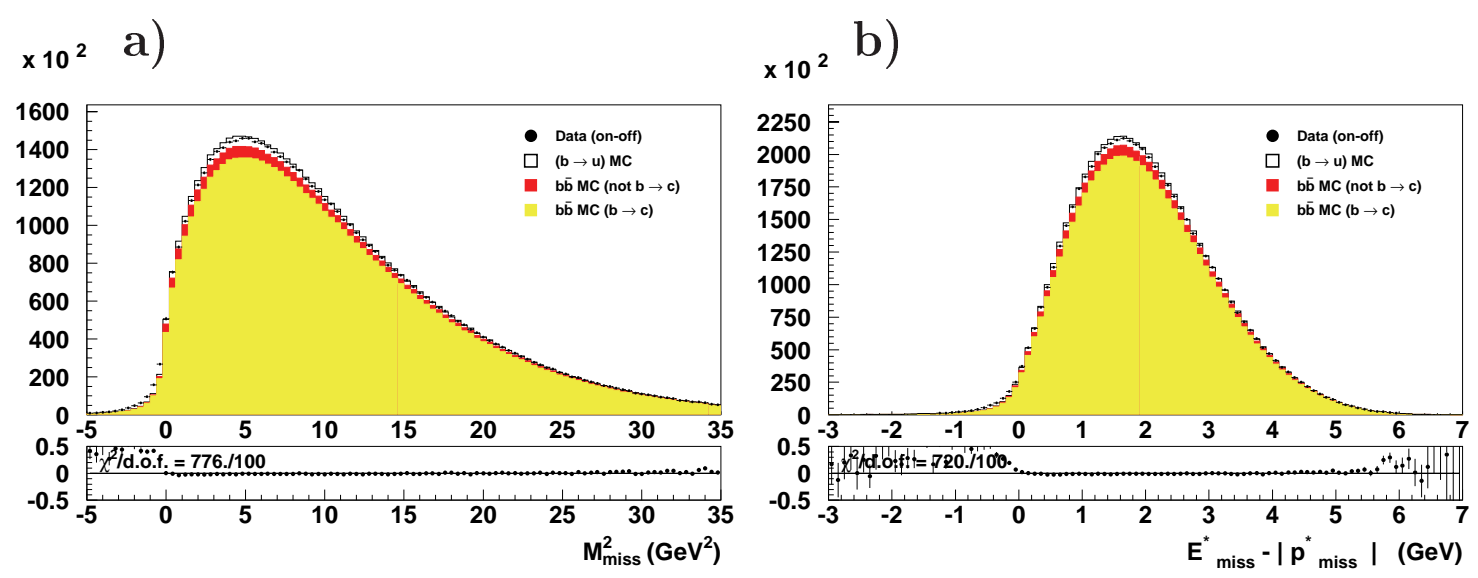

Figure 5.5: Data and Monte Carlo simulation distributions for a) the missing invariant mass squared $m_{\text {miss }}^{2}$ and b) $E_{\text {miss }}^{*}-\left|\vec{P}_{\text {miss }}^{*}\right|$ for events satisfying the requirements listed in section 5.2 only. No requirements are applied to $m_{\text {miss }}^{2}$ because it involves both physics $\left(E_{\text {miss }}^{*}+\left|\vec{P}_{\text {miss }}^{*}\right|\right)$ and resolution $\left(E_{\text {miss }}^{*}-\left|\vec{P}_{\text {miss }}^{*}\right|\right)$. The plots follow the same conventions as those of figure 5.1 .

of the bias. No dedicated $K_{L}^{0}$ veto is used since the efficiency in identifying these particles is very low. Secondary neutrinos are partially removed by requiring that the signal electron is the only identified lepton in the event. The remaining bias originates from sources such as shower leakage out of the EMC, misreconstructed particles, etc. The observed bias from Monte Carlo studies is shown in figures 5.6 and 5.7 for signal $B \rightarrow X_{u} e \bar{\nu}_{e}$ events after applying the selection requirements outlined in section 5.5. A linear function is fitted in the region $\left|\vec{P}_{\text {miss }}^{*}\right|<2.125 \mathrm{GeV}$ where about $\sim 75 \%$ of the events lie. This linear function is used to remove this bias and obtain an improved neutrino 4-vector estimate. The vector momentum is corrected according to

$$
\vec{P}_{\nu}^{*}=\vec{P}_{\text {miss }}^{*}\left(1-0.196+\frac{0.078 \mathrm{GeV}}{\left|\vec{P}_{\text {miss }}^{*}\right|}\right)
$$

and the 4-vector is constructed as

$$
p_{\nu}^{*}=\left(\vec{P}_{\nu}^{*},\left|\vec{P}_{\nu}^{*}\right|\right) .
$$



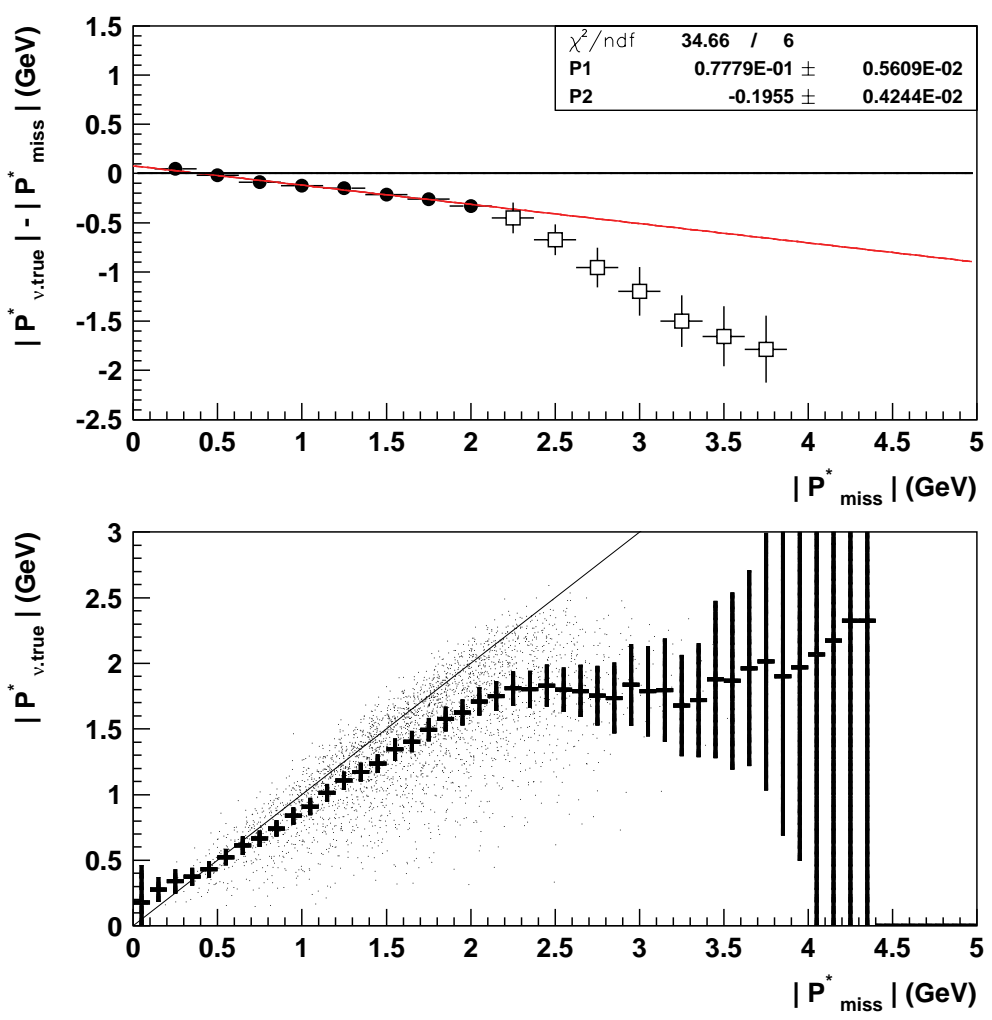

Figure 5.6: Generated $B \rightarrow X_{u} e \bar{\nu}_{e}$ neutrino momentum $\vec{P}_{\nu}^{*}$ true minus $\left|\vec{P}_{\text {miss }}^{*}\right|$ as a function of $\left|\vec{P}_{\text {miss }}^{*}\right|$ (top) and $\vec{P}_{\nu}^{*}$ true as a function of $\left|\vec{P}_{\text {miss }}^{*}\right|$ (bottom). In the latter plot, the mean $\vec{P}_{\nu}^{*}$ true value for $100 \mathrm{MeV}$ bin in $\left|\vec{P}_{\text {miss }}^{*}\right|$ is overlaid. The region $0 \mathrm{GeV}<\left|\vec{P}_{\text {miss }}^{*}\right|<2.125 \mathrm{GeV}$ where a linear fit applied is used to correct for the observed bias. Note that the selection requirements discussed in section 5.5 were applied to the events entering these plots.

As can be seen in figure 5.6, there is very little sensitivity to the true neutrino momentum for $\left|\vec{P}_{\text {miss }}^{*}\right|>2.125 \mathrm{GeV}$, which is why this region is ignored in computing the correction. Figure 5.7 displays the resolution on the neutrino reconstruction and $q^{2}$ for the signal simulation; clearly, the bias correction improves the $q^{2}$ resolution. Hence, the bias correction is used in computing $q^{2}$ and $s_{\mathrm{h}}^{\max }$ and results in the distributions shown in figure 5.8. Note that only the preselection requirements listed in section 5.2 were applied to the events entering these distributions. At this stage, the simulation appears to follow 
a)

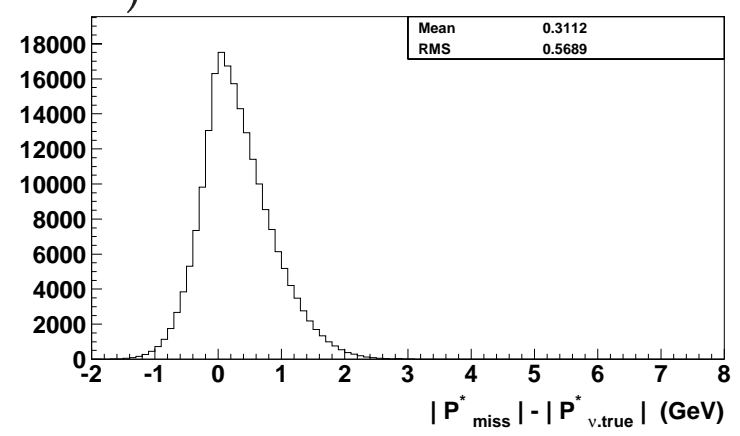

c)

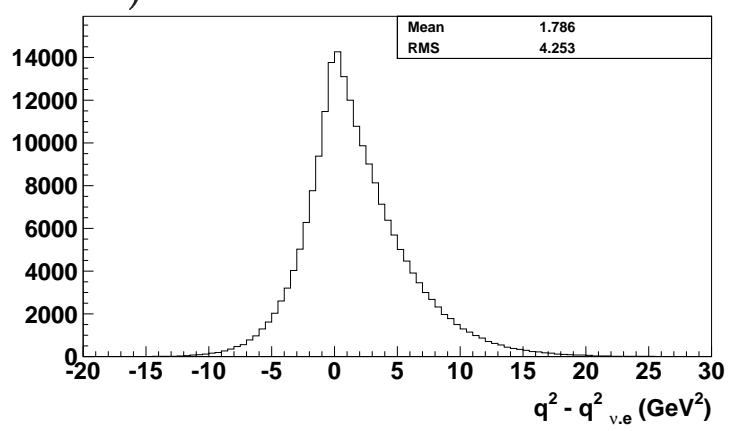

b)

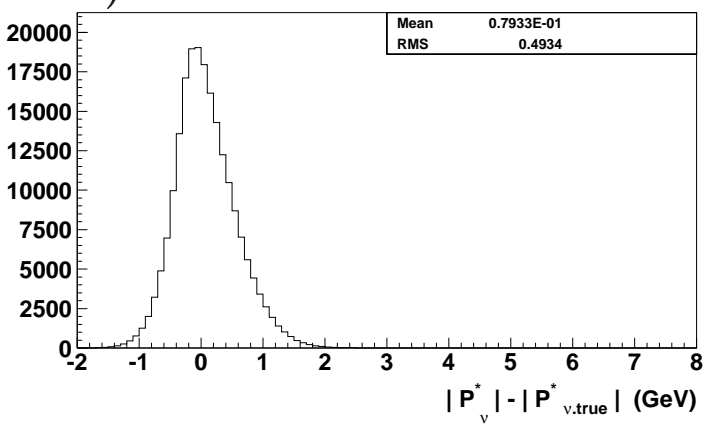

d)

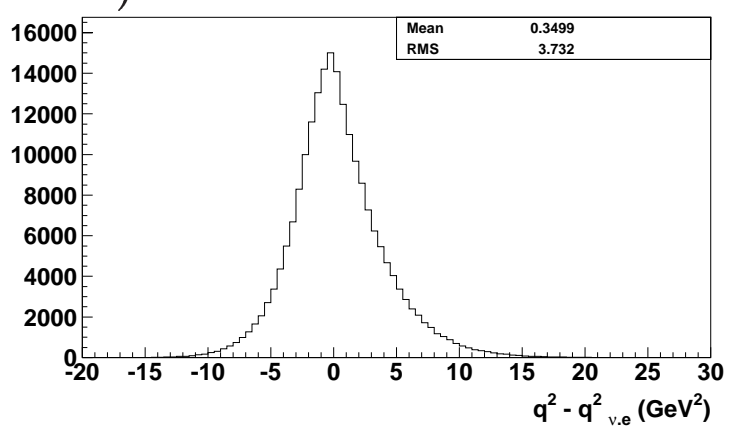

Figure 5.7: Signal $B \rightarrow X_{u} e \bar{\nu}_{e}$ distributions for a) the missing momentum resolution and b) the corrected neutrino momentum resolution; and $q^{2}$ resolution c) without and d) with the correction applied for the neutrino estimator. The resolution is computed as the reconstructed quantity minus the generated one. Note that these quantities were computed in the $\Upsilon(4 S)$ frame.

closely the data. A control sample will be used to further assess the quality of the neutrino reconstruction in section 6.3 .

\subsection{Refinement of the event selection}

Further requirements are applied to the events to reduce the amount of background, ensure a reasonable event reconstruction and hence improve the quality of the neutrino momentum measurement. To avoid introducing any bias in the analysis, the event selection is performed blind: only continuum (off-peak) data and $B \bar{B}$ Monte Carlo were used in optimizing the 

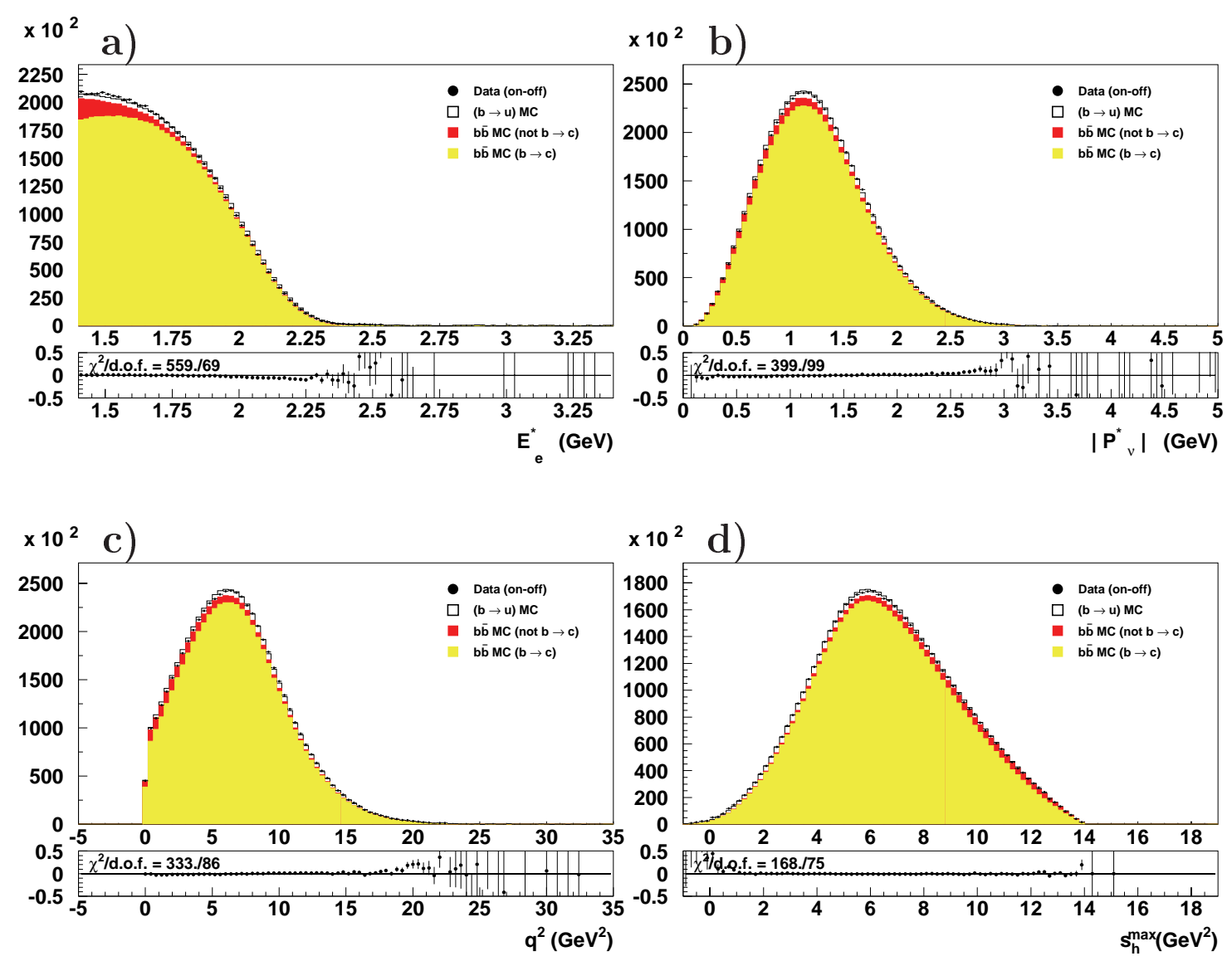

Figure 5.8: Comparison between data and Monte Carlo for the reconstructed a) electron energy,b) corrected neutrino momentum,c) $q^{2}$, and d) $s_{\mathrm{h}}^{\max }$. No further criteria than those listed in section 5.2 were applied to the events entering this figure. The plots follow the same conventions as those of figure 5.1. 
selection criteria. Selection requirements are applied for:

- A limit on the number of charged leptons in the event, $N_{\text {leptons }}$. This requirement aims at improving the neutrino resolution by reducing the number of events for which more than one neutrino is produced;

- A limit on the the magnitude of the missing momentum in the CM frame, $\left|\vec{P}_{\text {miss }}^{*}\right|$, to remove events where $\left|\vec{P}_{\text {miss }}^{*}\right|$ is a poor estimate of the neutrino momentum;

- A limit on $E_{\text {miss }}^{*}-\left|\vec{P}_{\text {miss }}^{*}\right|$, to improve the neutrino reconstruction and provide a reasonable agreement between the data and simulation (section 5.4);

- A fiducial limit on the missing energy vector in the $\Upsilon(4 S)$ frame, $\cos \theta_{\text {miss. }}^{*}$ This variable is also useful in suppressing continuum events as shown in figure 5.9;

- A limit on the angle between the thrust axis and the semileptonic electron momentum, $\cos \theta_{\vec{e} \cdot \vec{T}}^{*}$ in the $\Upsilon(4 S)$ frame. Similarly to the variable $R_{2}$ defined in section 5.2 , the thrust is an event shape variable which makes use of the $B \bar{B}$ topology to discriminate against continuum events as shown in figure 5.9. The thrust is generally defined as

$$
T_{R}=\left[\sum_{i=1}^{n} \overrightarrow{p_{i}} \cdot \vec{T} / \sum_{i=1}^{n} \overrightarrow{p_{i}}\right]_{\max },
$$

where $\vec{T}$ is the thrust axis. In this case, the semileptonic electron was not included in the computation of the thrust;

- A limit on the energy of the electron in the $\Upsilon(4 S)$ frame, $E_{e}^{*}$. An upper bound corresponding to the physical limit is set at $2.8 \mathrm{GeV}$. Furthermore, a lower bound was determined such that the statistical error on the measured $\mathcal{B}_{u}^{\mathrm{SL}}$ is optimal;

- Finally, a limit on $s_{\mathrm{h}}^{\max }$ is applied to further reduce the amount of $B \rightarrow X_{c} e \bar{\nu}_{e}$ backgrounds.

The selection criteria were optimized by finding a set of values for which the overall uncertainty on the extracted signal yield was minimal. The extracted signal in data, 
a)

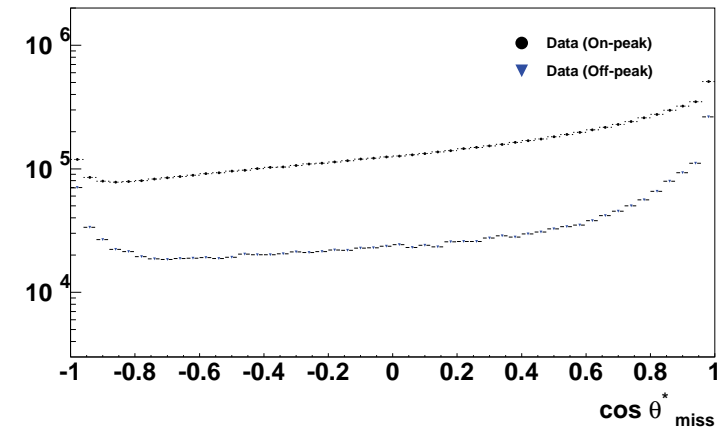

b)

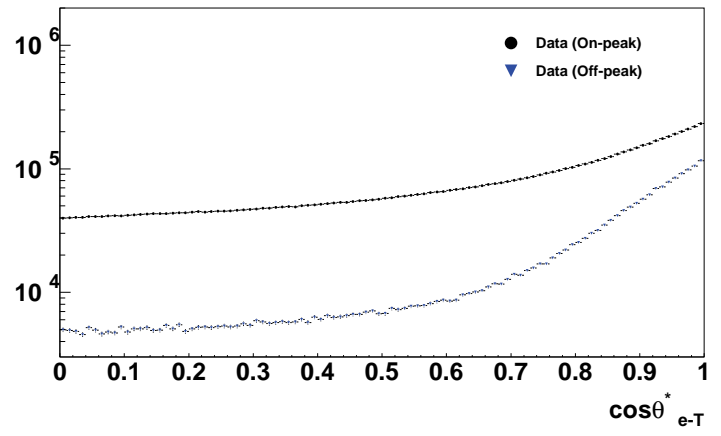

Figure 5.9: Distribution for a) $\cos \theta_{\text {miss }}^{*}$ and b) $\cos \theta_{\vec{e} \cdot \vec{T}}^{*}$ for on-peak and off-peak data. The off-peak data was scaled using the prescription described in section 5.1.1. The distributions sharply peak at \pm 1 for both variables. This property is used for suppressing continuum background events.

corrected for efficiency and acceptance, is

$$
S=\frac{N-B-r \cdot O}{\epsilon}
$$

where

- $N=\epsilon S+B+r \cdot O$ is the measured yield after the cuts;

- $B$ is the estimated background component ( $B \rightarrow X_{c} \ell \bar{\nu}_{\ell}$ events, cascades and fakes);

- $r \cdot O$ is the off-peak (continuum) data yield scaled to on-peak luminosity;

- $\epsilon$ is the efficiency times acceptance for $B \rightarrow X_{u} \ell \bar{\nu}_{\ell}$ signal events.

In the optimization procedure the yields corresponding to signal and $B \bar{B}$ background are taken from Monte Carlo (scaled to the number of $B \bar{B}$ pairs in data) while the off-peak data are used for evaluating the continuum yield. The systematic uncertainty in the predicted background is accounted for in the optimization by assigning an uncertainty $\delta B_{\mathrm{sys}}=\rho \cdot B$ where $\rho$ is a fixed parameter (discussed below). Hence, the overall uncertainty on the signal 
is approximately

$$
\begin{aligned}
\frac{\Delta S^{2}}{S^{2}} & =\frac{\delta N^{2}+\delta B^{2}+\delta(r \cdot O)^{2}}{(N-B-r \cdot O)^{2}}+\frac{\delta \epsilon^{2}}{\epsilon^{2}} \\
& =\frac{N+B+(\rho \cdot B)^{2}+r^{2} \cdot O}{(N-B-r \cdot O)^{2}}+\frac{\delta \epsilon^{2}}{\epsilon^{2}}
\end{aligned}
$$

where $r=8.41$ (defined in section 5.1.1), $O$ is the measured yield from off-peak data, $B$ is the estimated background from the simulation, $\rho$ is the fractional uncertainty in the background $B$ and $N-B-r \cdot O$ is the signal yield from the simulation. The fractional uncertainty on the signal yield due to $\rho$ is $\Delta S / S=\rho \cdot B / S$. The ratio $B / S$ in this analysis is approximately 1.2 , so choosing $\rho=0.05$ corresponds to a $6 \%$ uncertainty on $\Delta S / S$, which is roughly what is found in chapter 7 .

Figures 5.10 and 5.11 display the yield and relative uncertainty $\Delta S / S$ as a function of the selection criteria studied. For each plot, all selection criteria are held fixed except for the variable studied. The contributions from the statistical, background systematics and theoretical uncertainties are shown individually, and their sum computed in quadrature is also shown. Hence, the selection was optimized by adjusting each of the criteria and iterating the procedure until stable values were obtained. A description of the computation of the theoretical uncertainty is given in section 7.3. At this stage the theoretical uncertainties due to varying the two HQE parameters $m_{b}$ and $a$ are added in quadrature to the systematic and statistical uncertainties. The resulting selection criteria are presented in table 5.1. It's worth nothing that the upper bound obtained for $s_{\mathrm{h}}^{\max }$ using the optimization procedure is $3.5 \mathrm{GeV}^{2} \simeq m_{D^{0}}^{2}$. The effect of charge imbalance was also studied but no improvement was found by requiring an overall event charge of zero. ${ }^{7}$

\footnotetext{
${ }^{7}$ The sum of the charge from all tracks should be zero.
} 
a)

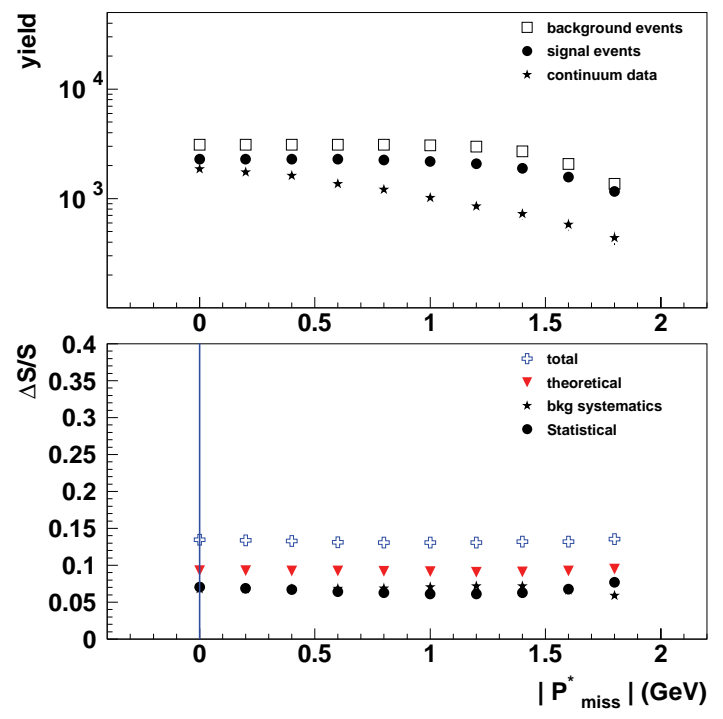

c)

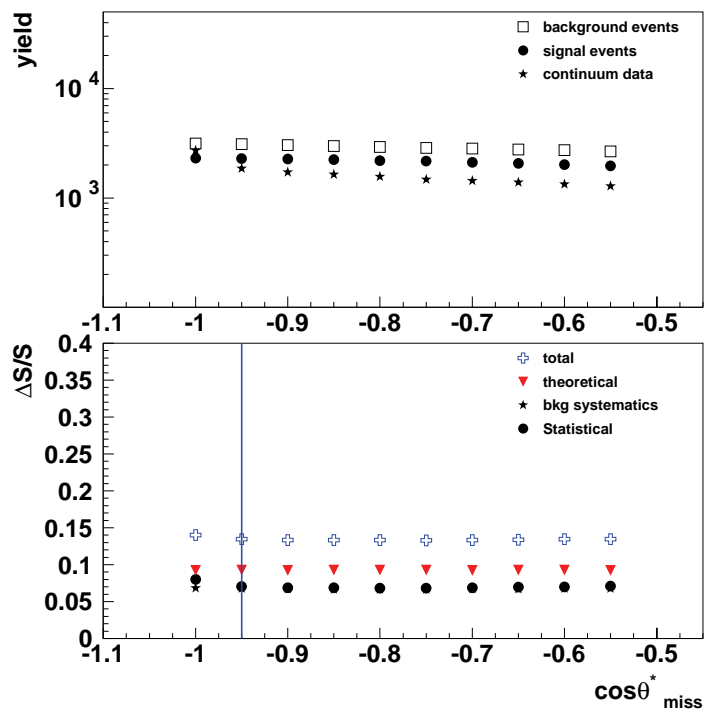

b)

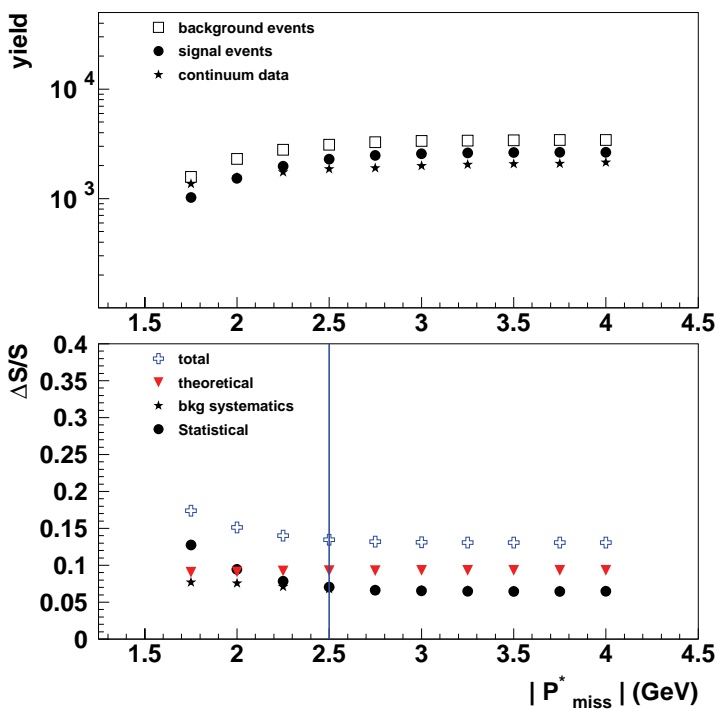

d)

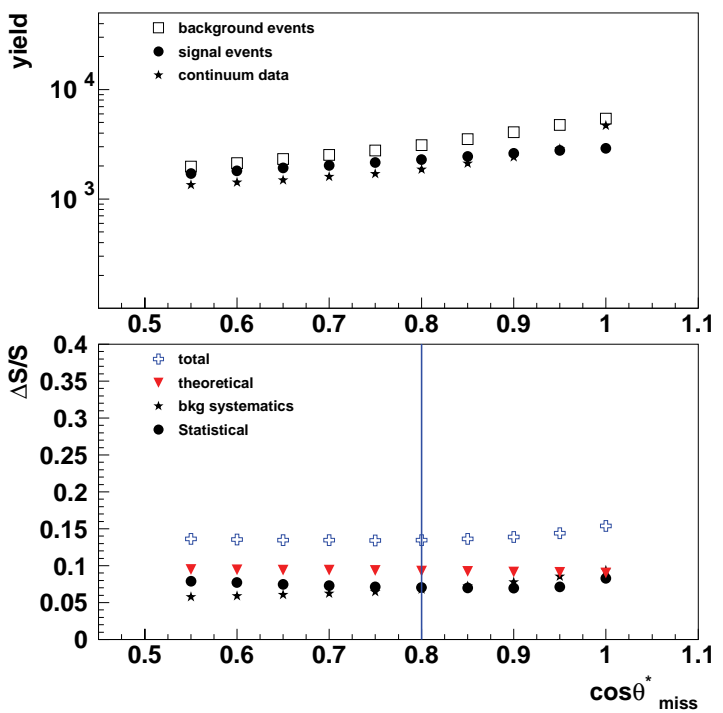

Figure 5.10: Optimization of event selection using the magnitude of the missing momentum (top) and the cosine of the missing momentum angle calculated in the $\Upsilon(4 S)$ frame. The vertical lines denote the lower (left) and upper (right) bounds chosen for the selection criteria. The upper plots display the yields for background, signal and continuum events, where as the lower plots display the total fractional uncertaintiy $\Delta S / S$ due to statistical, background systematics and theoretical uncertainties. Note that all selection criteria are held fixed except for the variable studied. 
a)

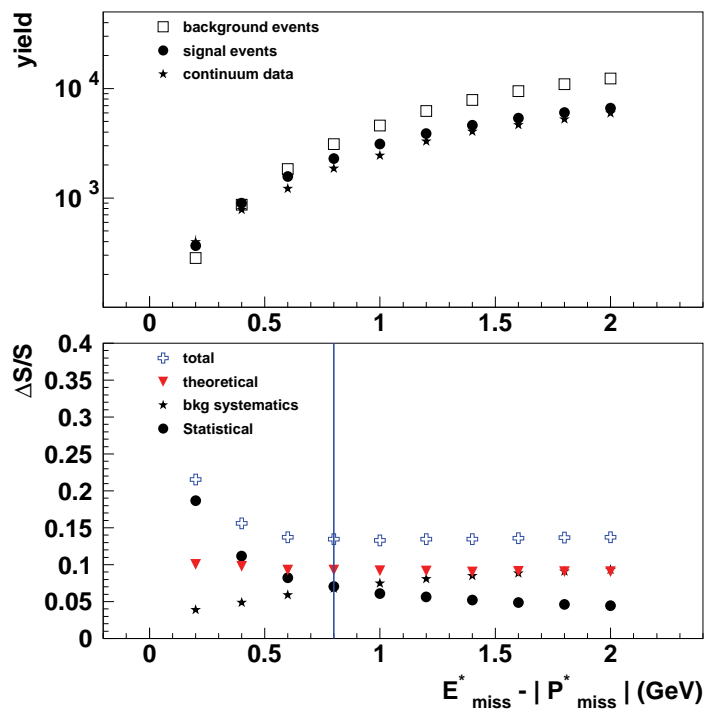

c)

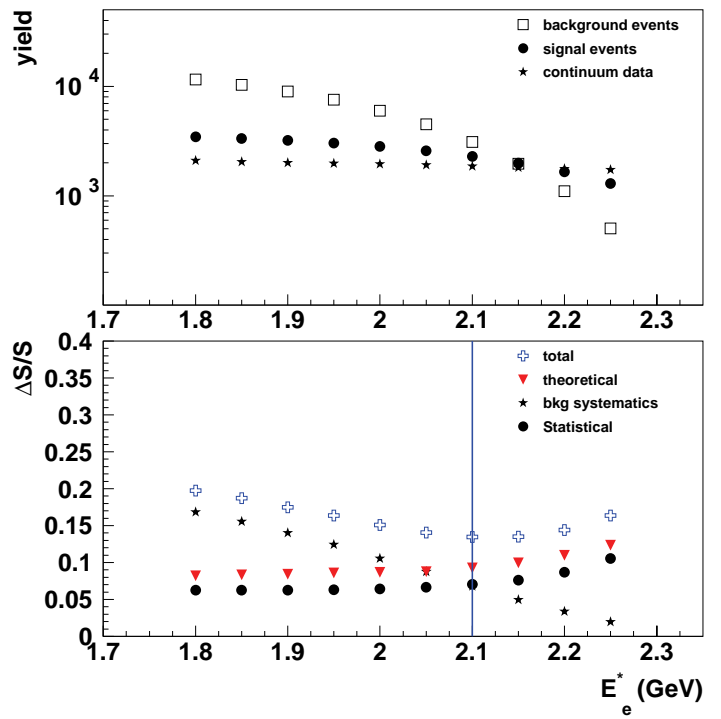

b)

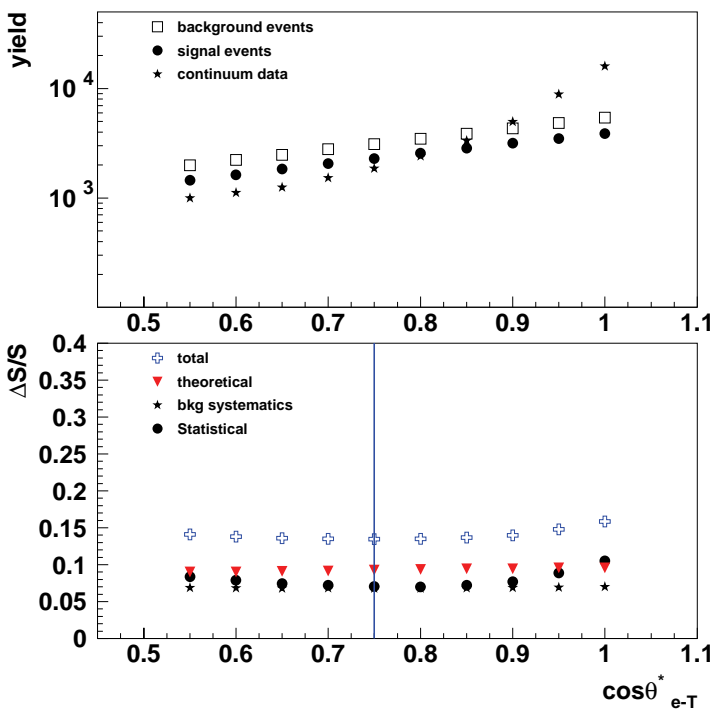

d)

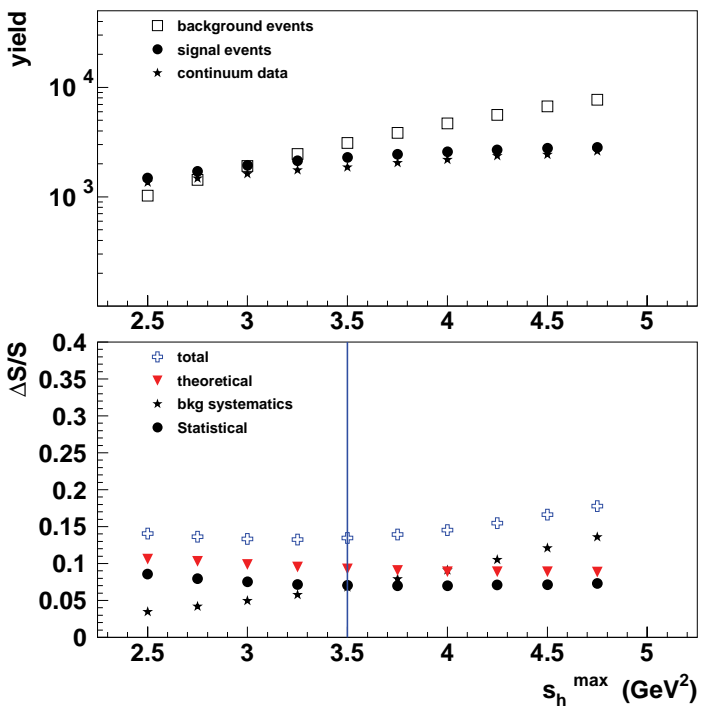

Figure 5.11: Optimization of the event selection using a) $E_{\text {miss }}^{*}-\left|\vec{P}_{\text {miss }}^{*}\right|$, b) $\cos \theta_{\vec{e} \cdot \vec{T}}^{*}$, c) $E_{e}^{*}$ and d) $s_{\mathrm{h}}^{\max }$. The vertical lines denote the requirement chosen for the selection criteria. The upper plots display the yields for background, signal and continuum events, where as the lower plots display the total fractional uncertaintiy $\Delta S / S$ due to statistical, background systematics and theoretical uncertainties. Note that all selection criteria are held fixed except for the variable studied. 


\begin{tabular}{|l|c|c|}
\hline Quantity & lower bound & upper bound \\
\hline \hline$N_{\text {leptons }}$ & 1 & 1 \\
$\left|\vec{P}_{\text {miss }}^{*}\right|$ & $0.0 \mathrm{GeV}$ & $2.5 \mathrm{GeV}$ \\
$E_{\text {miss }}^{*}-\left|\vec{P}_{\text {miss }}^{*}\right|$ & $0.0 \mathrm{GeV}$ & $0.8 \mathrm{GeV}$ \\
$\cos \theta_{\text {miss }}^{*}$ & -0.95 & 0.80 \\
$\cos \theta_{\vec{e} \cdot \vec{T}}^{*}$ & -0.75 & 0.75 \\
$E_{e}^{*}$ & $2.1 \mathrm{GeV}$ & $2.8 \mathrm{GeV}$ \\
$s_{\mathrm{h}}^{\text {max }}$ & none & $3.5 \mathrm{GeV}^{2}$ \\
\hline
\end{tabular}

Table 5.1: Refined event selection requirements as obtained by the optimization procedure.

\begin{tabular}{|l|r|r|r|r|}
\hline Criteria & $B \rightarrow X_{u} e \bar{\nu}_{e}$ & $B \rightarrow X_{c} e \bar{\nu}_{e}$ & Cascades & Fakes \\
\hline \hline Preselection & $97685 \pm 181$ & $2739770 \pm 1048$ & $121953 \pm 221$ & $7033 \pm 53$ \\
Bhabha veto & $96227 \pm 180$ & $2716443 \pm 1044$ & $120955 \pm 220$ & $7000 \pm 52$ \\
$N_{\text {leptons }}$ & $73598 \pm 157$ & $1937310 \pm 881$ & $70851 \pm 168$ & $3283 \pm 36$ \\
$\left|\vec{P}_{\text {miss }}^{*}\right|$ & $68068 \pm 151$ & $1854040 \pm 862$ & $68741 \pm 165$ & $3123 \pm 35$ \\
$E_{\text {miss }}^{*}-\left|\vec{P}_{\text {miss }}^{*}\right|$ & $11486 \pm 61$ & $215227 \pm 293$ & $8443 \pm 58$ & $354 \pm 11$ \\
$\cos \theta_{\text {miss }}^{*}$ & $8999 \pm 54$ & $161996 \pm 255$ & $5350 \pm 46$ & $209 \pm 9$ \\
$\cos \theta_{\vec{e} \cdot \vec{T}}^{*}$ & $5358 \pm 41$ & $95373 \pm 195$ & $2457 \pm 31$ & $94 \pm 6$ \\
\hline$E_{e}^{*}$ & $1339 \pm 19$ & $5033 \pm 44$ & $60 \pm 4$ & $9 \pm 1$ \\
$s_{\mathrm{h}}^{\max }$ & $1022 \pm 16$ & $1435 \pm 23$ & $23 \pm 3$ & $9 \pm 1$ \\
\hline
\end{tabular}

Table 5.2: Residual yields for $B^{0}$ decays after applying successively the selection requirements. The events of the simulation are scaled to the number of $B \bar{B}$ in data. Note that the last two requriements define the so-called signal region.

The impact of the selection and the resulting yields after applying successively the selection criteria to the simulation is illustrated in tables 5.2 and 5.3 for neutral and charged $B$ decays, respectively. Thus, about 2300 signal $B \rightarrow X_{u} e \bar{\nu}_{e}$ events and 3200 background events are expected to be selected from $B \bar{B}$ data. The difference in yields between $B^{+} B^{-}$ and $B^{0} \bar{B}^{0}$ at the preselection level is due to the difference in semileptonic branching fractions (see tables 4.2 and 4.4). The selection requirements amplifies this asymmetry slightly, since the efficiency for $B^{0} \bar{B}^{0}$ events is $10 \%$ smaller than for $B^{+} B^{-}$events. This is caused by the higher $D^{+}$content in $B^{0}$ decays. In fact, $D^{+}$decays more often than $D^{0}$ to neutrinos and $K_{L}^{0}$ (see table 7.4), and such events are therefore more likely to fail the neutrino reconstruction requirements. 


\begin{tabular}{|l|r|r|r|r|}
\hline Criteria & $B \rightarrow X_{u} e \bar{\nu}_{e}$ & $B \rightarrow X_{c} e \bar{\nu}_{e}$ & Cascades & Fakes \\
\hline \hline Preselection & $109295 \pm 187$ & $2917330 \pm 1028$ & $103114 \pm 193$ & $6786 \pm 49$ \\
Bhabha veto & $107977 \pm 186$ & $2896460 \pm 1024$ & $102587 \pm 192$ & $6763 \pm 49$ \\
$N_{\text {leptons }}$ & $84167 \pm 164$ & $2133490 \pm 879$ & $61126 \pm 148$ & $3561 \pm 35$ \\
$\left|\vec{P}_{\text {miss }}^{*}\right|$ & $78071 \pm 158$ & $2044380 \pm 860$ & $59175 \pm 146$ & $3396 \pm 35$ \\
$E_{\text {miss }}^{*}-\left|\vec{P}_{\text {miss }}^{*}\right|$ & $14557 \pm 67$ & $288078 \pm 323$ & $9082 \pm 57$ & $559 \pm 14$ \\
$\cos \theta_{\text {miss }}^{*}$ & $11396 \pm 59$ & $217863 \pm 280$ & $5720 \pm 45$ & $337 \pm 11$ \\
$\cos \theta_{\vec{e} \cdot \vec{T}}^{*}$ & $6825 \pm 46$ & $129552 \pm 216$ & $2677 \pm 31$ & $141 \pm 7$ \\
\hline$E_{e}^{*}$ & $1684 \pm 21$ & $6628 \pm 49$ & $68 \pm 4$ & $0 \pm 0$ \\
$s_{\mathrm{h}}^{\text {max }}$ & $1274 \pm 17$ & $1678 \pm 24$ & $30 \pm 3$ & $0 \pm 0$ \\
\hline
\end{tabular}

Table 5.3: Residual yields for $B^{+}$decays after applying successively the selection requirements. The events of the simulation are scaled to the number of $B \bar{B}$ in data. Note that the last two requriements define the so-called signal region.

\subsection{Comparison between data and simulation}

In this section, kinematical distributions for data and Monte Carlo are compared to validate the simulation. Since the dominant systematic uncertainties are not included in these comparisons, the following is mostly a qualitative crosscheck. Figures 5.12 to 5.14 present spectra for various quantities satisfying the refined event selection listed in section 5.5; all of the criteria listed therein were applied in producing the plots except when a cut corresponded to the variable plotted, in which case the selected region is denoted by an arrow. As shown in figure 5.12, the event requirements do not introduce any discrepancy between data and the simulation in terms of the event multiplicity and energy reconstruction when comparing with the results shown in figures 5.1 and 5.2. The particle selection also results in a net improvement in $\chi^{2}$ probability.

Similarly, reasonable agreement between the data and Monte Carlo simulation is achieved for the neutrino reconstruction in terms of the missing energy and momentum, $E_{\text {miss }}^{*}-\left|\vec{P}_{\text {miss }}^{*}\right|$, and $m_{\text {miss }}^{2}$, as presented in figure 5.13. The sharpness of the latter quantity is a result of the $E_{\text {miss }}^{*}-\left|\vec{P}_{\text {miss }}^{*}\right|$ and $\left|\vec{P}_{\text {miss }}^{*}\right|$ requirements. The variables $\cos \theta_{\text {miss }}^{*}$ and $\cos \theta_{\vec{e} \cdot \vec{T}}^{*}$ used in suppressing continuum events also display a reasonable agreement within the selected region, and yield $\chi^{2}$ probabilities of $19 \%$ and $32 \%$, respectively. Figure 5.14 


\begin{tabular}{|l|r|r|r|r|}
\hline Criteria & On-peak data & Off-peak data & $B \bar{B}$ data & $B \bar{B}$ Monte Carlo \\
\hline \hline Preselection & $9794431 \pm 3129$ & $3854240 \pm 5693$ & $5940190 \pm 6497$ & $6102970 \pm 1521$ \\
Bhabha veto & $7722665 \pm 2778$ & $1844300 \pm 3938$ & $5878370 \pm 4820$ & $6054420 \pm 1515$ \\
$N_{\text {leptons }}$ & $5992187 \pm 2447$ & $1591610 \pm 3658$ & $4400572 \pm 4402$ & $4367390 \pm 1286$ \\
$\left|\vec{P}_{\text {miss }}^{*}\right|$ & $5538573 \pm 2353$ & $1347200 \pm 3366$ & $4191370 \pm 4107$ & $4179002 \pm 1258$ \\
$E_{\text {miss }}^{*}-\left|\vec{P}_{\text {miss }}^{*}\right|$ & $825651 \pm 908$ & $290524 \pm 1563$ & $535127 \pm 1808$ & $547788 \pm 453$ \\
$\cos \theta_{\text {miss }}^{*}$ & $608556 \pm 780$ & $206114 \pm 1316$ & $402442 \pm 1530$ & $411874 \pm 393$ \\
$\cos \theta_{\vec{e} \cdot \vec{T}}^{*}$ & $261854 \pm 511$ & $21724 \pm 427$ & $240129 \pm 666$ & $242480 \pm 301$ \\
\hline$E_{e}^{*}$ & $17446 \pm 132$ & $3524 \pm 172$ & $13921 \pm 216$ & $14824 \pm 72$ \\
$s_{\mathrm{h}}^{\text {max }}$ & $6989 \pm 83$ & $1858 \pm 125$ & $5130 \pm 150$ & $5473 \pm 42$ \\
\hline
\end{tabular}

Table 5.4: Yields after applying the event requirements successively for data and Monte Carlo simulation. Note that the errors shown are purely statistical.

displays the electron energy spectrum, the corrected neutrino momentum and the resulting $q^{2}$ and $s_{\mathrm{h}}^{\max }$ distributions. In the latter, the signal $s_{\mathrm{h}}^{\max }<3.5 \mathrm{GeV}^{2}$ as well as the sideband $s_{\mathrm{h}}^{\max }>4.25 \mathrm{GeV}^{2}$ regions are denoted by the arrows, and separate $\chi^{2}$ probabilities are computed for each region. Overall, a reasonable agreement is achieved between data and simulation within the selected phase-space regions, with $\chi^{2}$ probabilities of $29 \%$ or better. The apparent discrepancies observed at $E_{e}^{*}<1.9 \mathrm{GeV}$ are well within background systematics as will be discussed in section 8.1. Furthermore, large theoretical uncertainties reside in the signal simulation which affect the details of the spectra displayed in this section. Table 5.4 summarizes the yields for data prior to and after continuum subtraction, and compares these results with the expected yields from Monte Carlo. For each selection criteria, comparable yields and relative efficiencies are obtained for $B \bar{B}$ data and Monte Carlo. Note that there is a $1.1 \%$ uncertainty in the number of $B \bar{B}$ events from our knowledge of the luminosity and $B \bar{B}$ cross-section, which will be discussed in section 7.13. 
a)

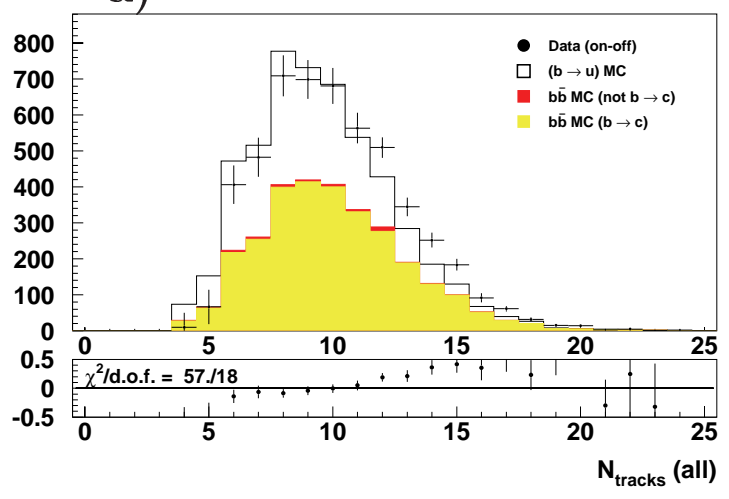

c)

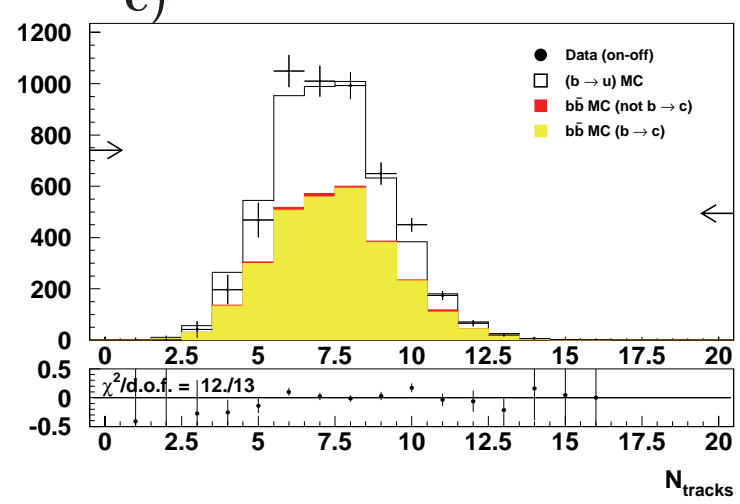

e)

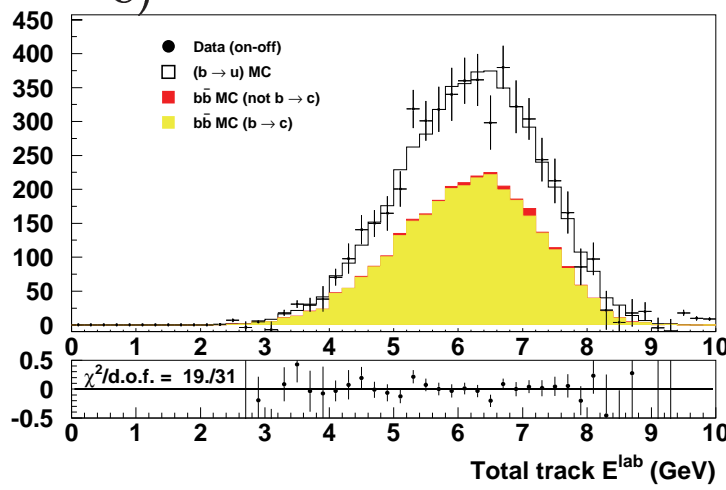

b)

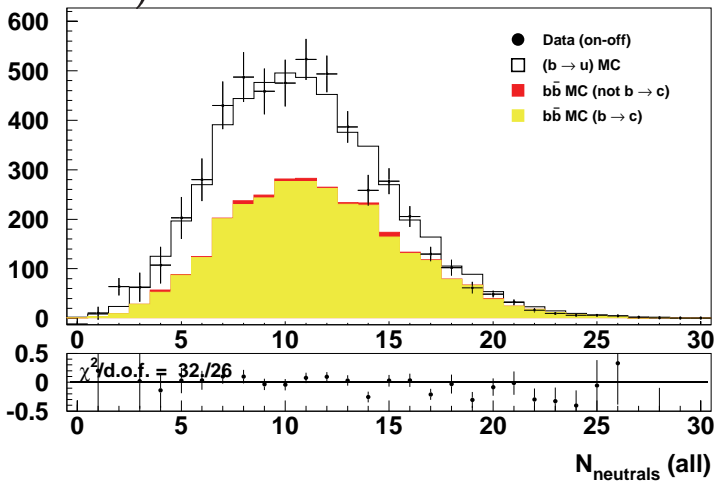

d)

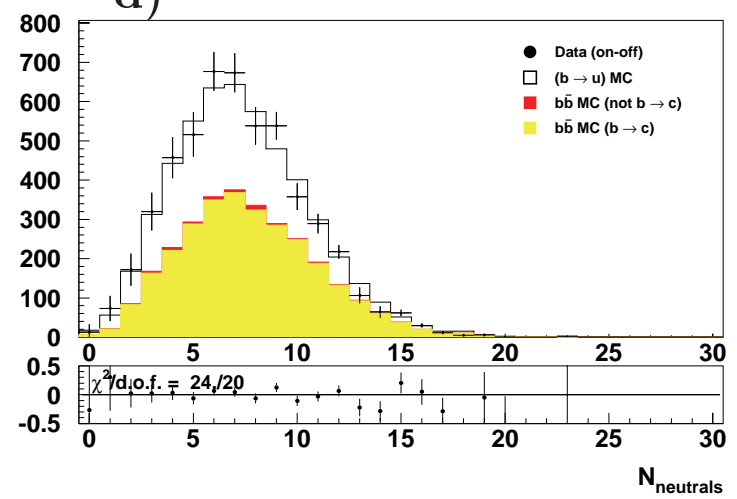

Figure 5.12: Data and MC distributions displaying the raw multiplicity for a) charged tracks and b) neutral clusters; also shown in c) and d) are the corresponding multiplicities after applying the particle selection described in section 5.3.2 and section 5.3.3, respectively. The resulting total energy distributions reconstructed from e) charged track and f) neutral clusters in the laboratory frame are displayed. The events satisfy the refined selection described in section 5.5. The plots follow the same conventions as those of figure 5.1. 
a)

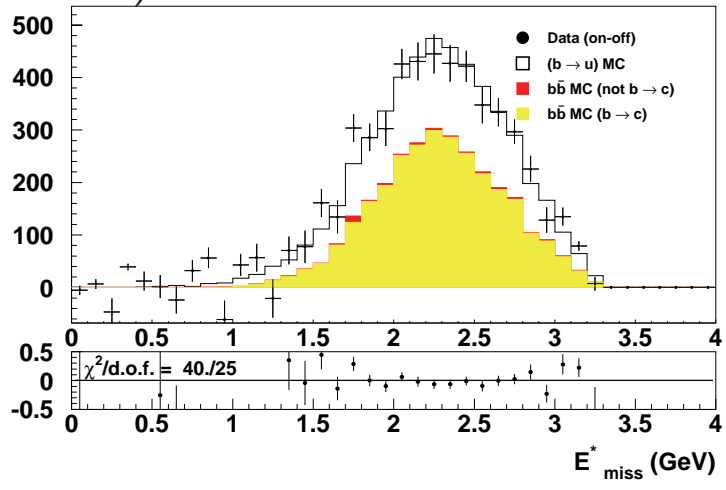

c)

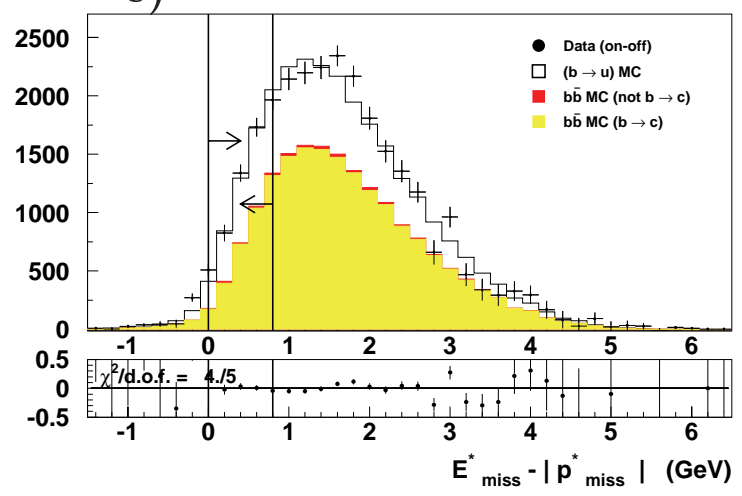

e)

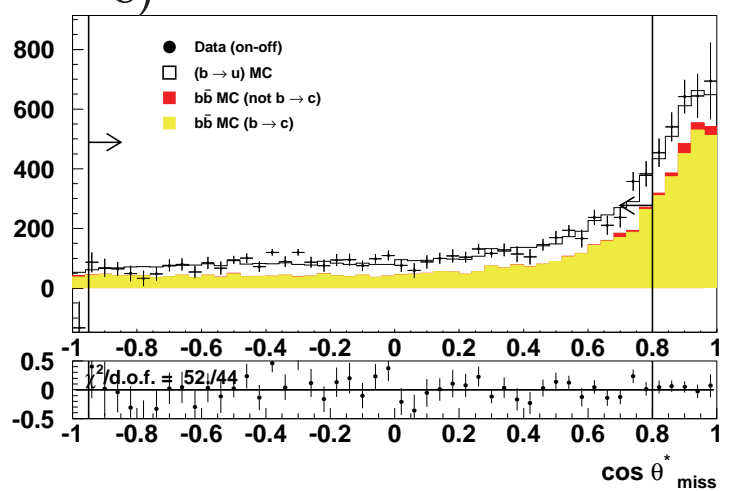

b)

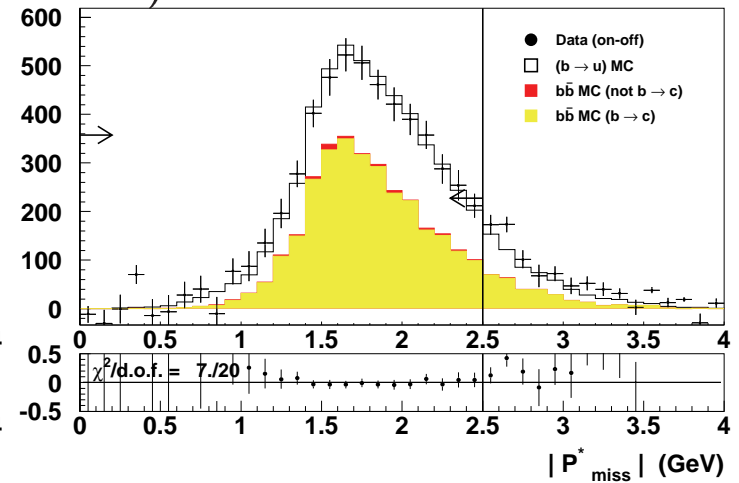

d)

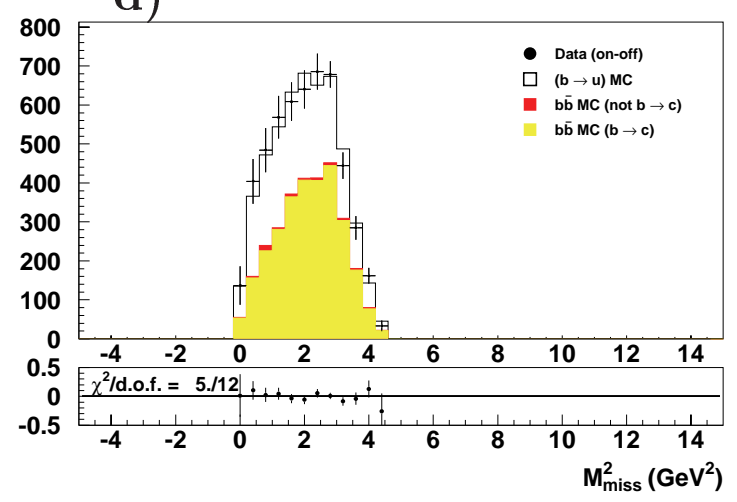

f)

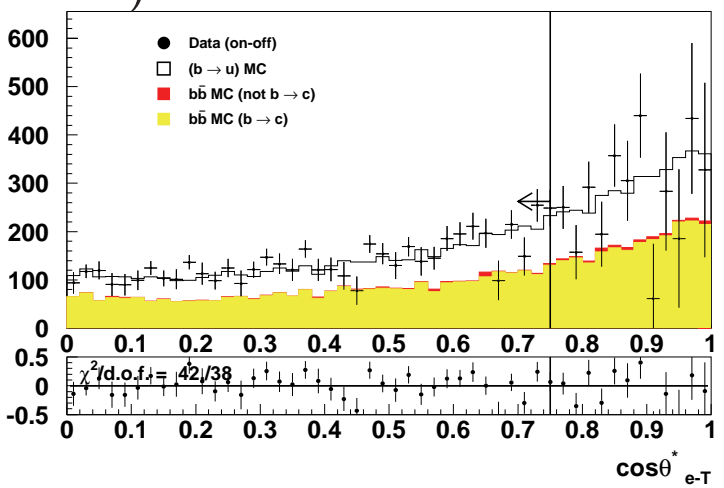

Figure 5.13: Data and Monte Carlo distributions computed in the $\Upsilon(4 S)$ frame for a) $E_{\text {miss }}^{*}$, b) $\left|\vec{P}_{\text {miss }}^{*}\right|$, c) $E_{\text {miss }}^{*}-\left|\vec{P}_{\text {miss }}^{*}\right|$, d) $m_{\text {miss }}^{2}$ e) $\cos \theta_{\text {miss }}^{*}$ and f) $\cos \theta_{\vec{e} \cdot \vec{T}}^{*}$ for events satisfying the refined selection described in section 5.5. The plots follow the same conventions as those of figure 5.1. 
a)

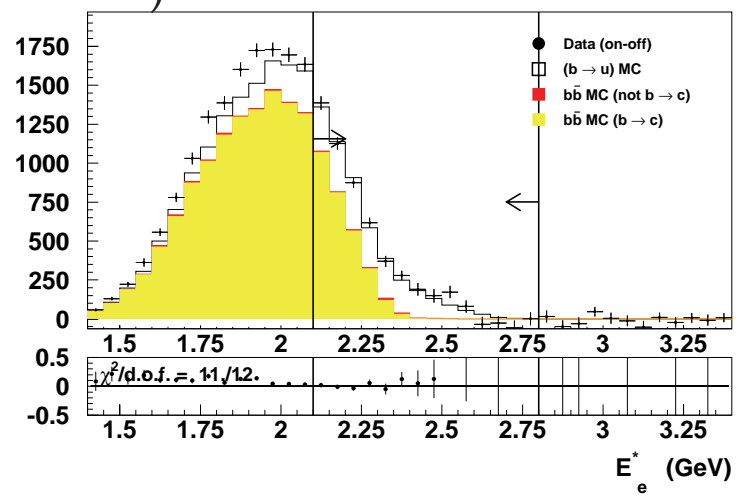

c)

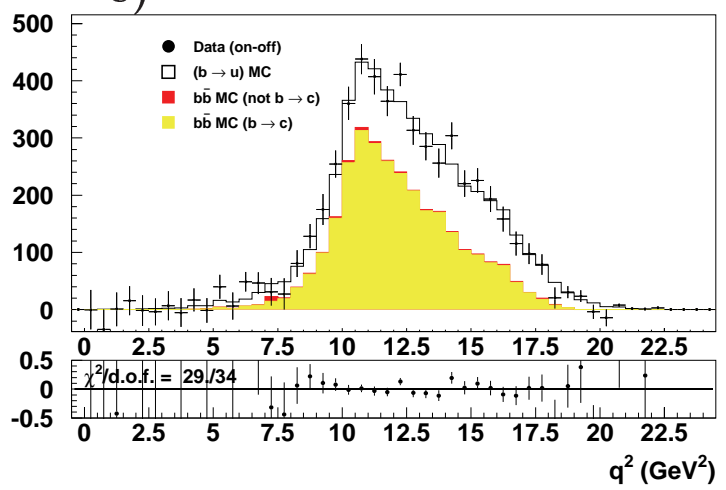

b)

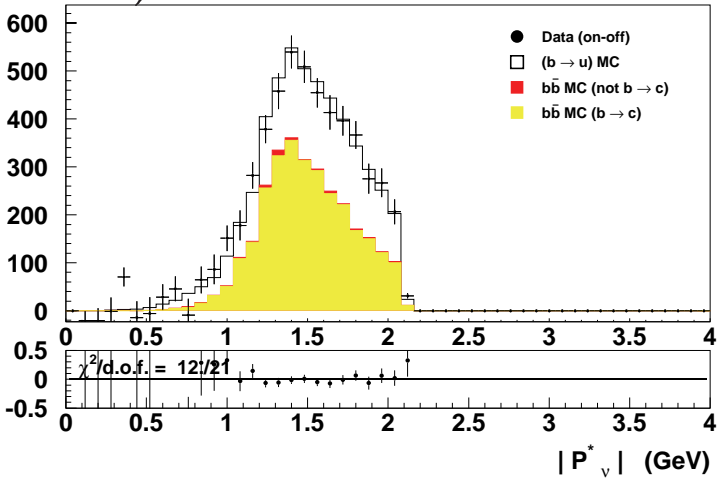

d)

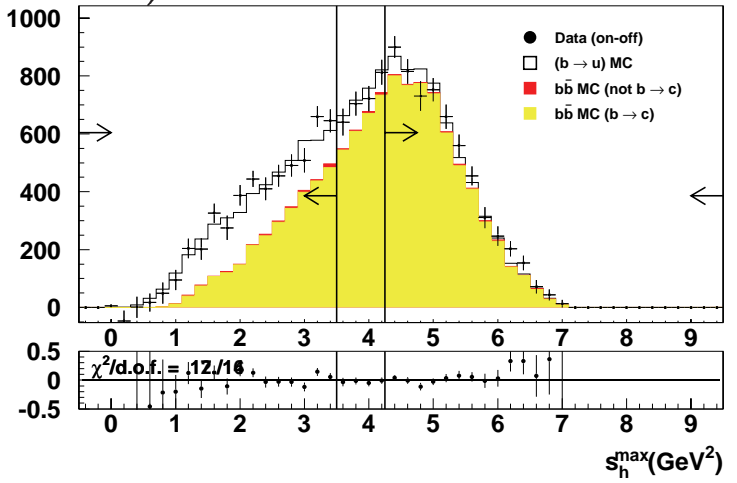

Figure 5.14: Distributions computed in the $\Upsilon(4 S)$ frame for a) $E_{e}^{*}$, b) $\cos \theta_{\vec{e} \cdot \vec{T}}^{*}$, c) $q^{2}$ and, d) $s_{\mathrm{h}}^{\max }$ for events satisfying the refined selection described in section 5.5. The plots follow the same conventions as those of figure 5.1. 


\section{Chapter 6}

\section{Control Sample Studies}

Testis unus, testis nullus

The event selection requirements listed in the previous sections make the efficiency predictions sensitive to the details of the Monte Carlo simulation. It is thereful useful to consider an independent control sample for which the effects of the selection criteria on the agreement between data and simulation can be studied. The Monte Carlo efficiency can then be corrected using this control sample such that it reproduces the data selection efficiency more accurately.

For these purposes the decay chain $B \rightarrow D^{0} e \bar{\nu}_{e} X$, where the $D^{0}$ is reconstructed in the $K^{-} \pi^{+}$decay mode, is studied. The kinematic requirements outlined in section 6.1 are applied to improve the purity of the sample and result in $X$ typically being null or a soft pion or photon from a $D^{*}$. These decays have a topology similar to the $B \rightarrow X_{u} e \bar{\nu}_{e}$ signal events:

- No additional neutrinos or neutral hadrons are present in the decay chain;

- The decay of the other $B$ is unbiased by the $B \rightarrow D^{0} e \bar{\nu}_{e} X$ selection procedure and thus identical to the other $B$ in $B \rightarrow X_{u} e \bar{\nu}_{e}$ signal events.

The latter item is particularly important. It means that this control sample can be used to correct for deficiencies in the simulation of the decay of the other $B$ meson, and in the 
simulation of the reconstruction of the tracks and clusters that come from it.

Section 6.2 describes how the control sample is also used to improve the modeling of the $B \rightarrow X_{c} e \bar{\nu}_{e}$ decays. The updated Monte Carlo is compared to data following the procedure used in section 5.6. The quality of the neutrino reconstruction is then investigated using the $D^{0} e \bar{\nu}_{e}$ decays in section 6.3. Finally, a detailed study of the selection efficiency is presented in section 6.5 and a correction factor for the signal simulation is obtained.

\subsection{Selecting $B \rightarrow D^{0} e \bar{\nu}_{e}$ events}

The selection of $B \rightarrow D^{0} e \bar{\nu}_{e}$ decays is based on an earlier study [64] and is summarized below. $D^{0}$ mesons are first reconstructed by studying all possible combinations of charged tracks consistent with the decay of a $D^{0}$ to a $K^{-} \pi^{+}$pair. A vertex fit is performed and combinations for which the $\chi^{2}$ probability is above $0.1 \%$ are retained. The pairs of tracks are then required to have an invariant mass within $40 \mathrm{MeV}$ of the nominal $D^{0}$ mass of $1865 \mathrm{MeV}$ [18]. This rather wide acceptance region within the invariant mass is applied to allow events to populate the "sideband" regions of the $D^{0}$ mass spectrum. These sideband samples are then used in order to study background contributions from both continuum and $B$ decays. Finally, the $D^{0}$ meson is required to have a momentum $\left|\vec{p}_{D^{0}}^{*}\right|>0.5 \mathrm{GeV}$.

Candidate $B \rightarrow D^{0} e \bar{\nu}_{e}$ decays are identified by combining reconstructed $D^{0}$ with the signal electron. Further criteria are imposed to the $D^{0} e$ combination to ensure they originated from $B \rightarrow D^{0} e \bar{\nu}_{e} X$ decays:

1. The electron energy must be $>2.0 \mathrm{GeV}$. Note that this requirement is relaxed to $E_{e}^{*}>1.4 \mathrm{GeV}$ in sections 6.2 and 6.3 in order to have sufficent statistics;

2. The vertex fit probability is $>0.1 \%$;

3. The invariant mass of the $D^{0} e$ system is $m_{D^{0} e}>3.0 \mathrm{GeV}$;

4. Finally, the angle between the $B$ and the $D^{0} e$ combination is required to satisfy $-2.5<\cos \theta_{B, D^{0} e}<1.1$. 
Note that the event preselection described in section 5.2 is also applied to the control sample. The quantity $\cos \theta_{B, D^{0} e}$ is not measured directly since there is no handle on the direction of the $B$ meson. Instead, it is calculated under the assumption that the only unreconstructed particle from the $B$ is a massless neutrino. The energy $E_{B}^{*}$ and magnitude of the momentum $\left|p_{B}^{*}\right|$ of the $B$ meson are determined in the center-of-mass frame using the invariant mass of the $e^{+} e^{-}$beams and the $B$ meson mass as

$$
E_{B}=\frac{1}{2} \sqrt{\left(p_{e^{+}}+p_{e^{-}}\right)^{2}}
$$

and

$$
\left|p_{B}^{*}\right|=\sqrt{E_{B}^{2}-m_{B}^{2}}
$$

respectively. The angle between the $D^{0} e$ and the $B$ meson is defined as

$$
\cos \theta_{B, D^{0} e}=\vec{p}_{B} \cdot \vec{p}_{D^{0} e} /\left|\vec{p}_{B}\right|\left|\vec{p}_{D^{0} e}\right|
$$

If one asumes that the only particle missing is a neutrino, it follows that

$$
\cos \theta_{B, D^{0} e}=\frac{2 E_{B}^{*} E_{D^{0} e}^{*}-m_{\mathrm{B}}^{2}-m_{D^{0} e}^{2}}{2\left|p_{B}^{*}\right|\left|p_{D^{0} e}^{*}\right|} .
$$

If this is truly the case, then $\cos \theta_{B, D^{0} e}$ should lie in the physical \pm 1 range. The asymmetry in the $\cos \theta_{B, D^{0} e}$ requirement is designed to provide a good efficiency for both the direct $B \rightarrow D^{0} e \bar{\nu}_{e}$ decay and for decays of the type $B \rightarrow X_{c} e \bar{\nu}_{e}$ where $X_{c}$ is a relatively low-mass charm system, such as a $D^{\star}$, that produces a $D^{0}$. The latter tend to produce lower negative values, as can be seen in figure 6.1. The unphysical upper constraint on $\cos \theta_{B, D^{0} e}$ of 1.1 is to account for any reconstruction and detector effects that may feed into the calculation. The limits on $\cos \theta_{B, D^{0} e}$ are further relaxed in section 6.2 to adjust the $B \rightarrow X_{c} e \bar{\nu}_{e}$ in the simulation.

The control sample cannot be expected to correct for deficiencies in the simulation of the semileptonically decaying $B$ meson, since it is biased (the daughters of the $D^{0}$ are required to be reconstructed). However, to the extent that the multiplicity of the control sample is similar to that for signal $B \rightarrow X_{u} e \bar{\nu}_{e}$ decays, as shown in figure 6.2 ), detector 


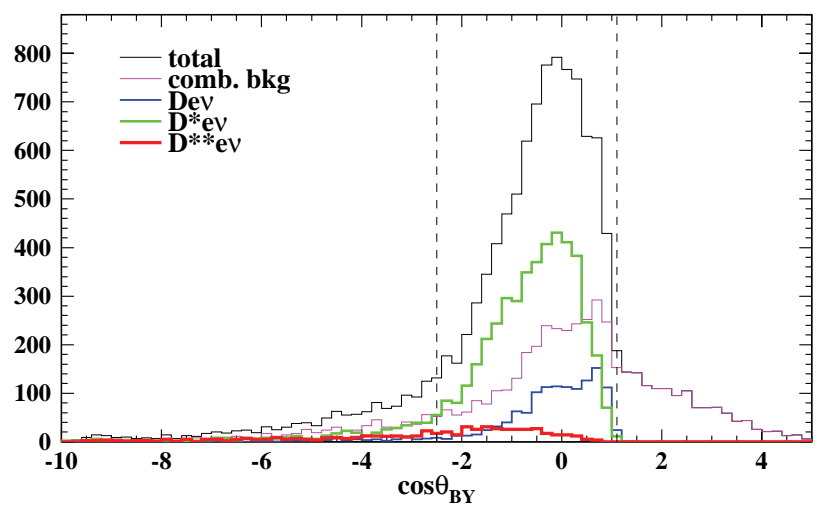

Figure 6.1: The distribution of $\cos \theta_{B, Y}$ (where $Y$ refers to the $D^{0} e$ system) in the $D^{0} e \bar{\nu}_{e}$ control sample for candidates in the $D^{0}$ mass window, showing the contributions from direct $B \rightarrow D^{0} e \bar{\nu}_{e}$ decays and feeddown from higher mass charm states. The combinatorial component can be subtracted using $D^{0}$ mass sidebands.

modeling uncertainties are similar between the signal and control sample even for $B$ mesons decaying semileptonically.

\subsection{Adjusting background branching ratios}

The $B \rightarrow D^{0} e \bar{\nu}_{e} X$ control sample consists of partially reconstructed semileptonic decays where what is missing is typically nothing or a soft transition particle. In relaxing the cuts on $\cos \theta_{B, D^{0} e}$ the sample then includes feeddown from all higher mass $B \rightarrow X_{c} e \bar{\nu}_{e}$ decays, allowing its use in tuning the modeling of semileptonic $b \rightarrow c$ decays to the data. The three variables $E_{e}^{*}, p_{D}^{*}$ and $\cos \theta_{B, D^{0} e}$ provide useful discrimination between the direct $B \rightarrow D^{0} e \bar{\nu}_{e}$ decays, the $B \rightarrow D^{*} e \bar{\nu}_{e}$ decays, and other sources of $D^{0} e \bar{\nu}_{e}$ combinations, namely cases where the electron comes from a $B \rightarrow D^{* *} e \bar{\nu}_{e}$ or $B \rightarrow D(*) \pi e \bar{\nu}_{e}$ decay $(\sim 80 \%$ of the cases $)$, is a fake or non-primary electron $(\sim 15 \%)$ or where the electron and $D^{0}$ don't come from the same semileptonic $B$ decay $(\sim 5 \%)$. For this purpose, a 3-dimensional distribution is formed with binning:

- $p_{D}^{*}: 0.5,1.0,1.5,2.0,2.5 \mathrm{GeV}$

- $E_{e}^{*}: 1.4,1.6,1.8,2.0,2.2,2.5 \mathrm{GeV}$ 


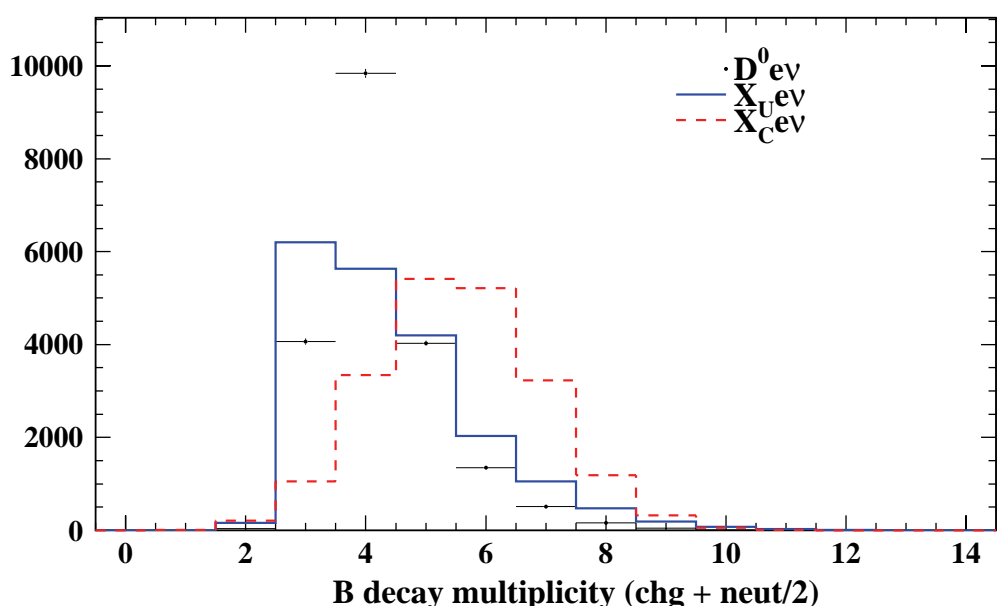

Figure 6.2: Multiplicity, defined as $N_{\text {chg }}+N_{\text {neut }} / 2$, for the $B \rightarrow D^{0} e \bar{\nu}_{e} X$ control sample (points), for $B \rightarrow X_{u} e \bar{\nu}_{e}$ decays (solid histogram) and for $B \rightarrow X_{c} e \bar{\nu}_{e}$ decays (dashed histogram), all normalized to equal area.

- $\cos \theta_{B, D^{0} e^{:}}-10,-2.5,-1.1,1.1,5$

This 80 bin array is chosen to exploit the features of the underlying components while keeping the overall number of bins small enough to allow meaningful statistics in each bin.

The Monte Carlo distributions ${ }^{1}$ are shown in figure 6.3 for $D^{0} e \bar{\nu}_{e}$ candidates satisfying the event preselection cuts. The 3 - $\mathrm{d}$ information is represented as a 1-dimensional histogram of 80 bins with structure $i+j * 5+k * 20$, where $i, j$ and $k$ are the bin indices for $p_{D}^{*}, E_{e}^{*}$ and $\cos \theta_{B, D^{0} e}$, respectively. As can be seen in Figure 6.4 , the $B \rightarrow D^{0} e \bar{\nu}_{e}$, $B \rightarrow D^{*} e \bar{\nu}_{e}$ and remaining components differ in shape, and the sum of these histograms is compared with the data. Note that the overall normalization in this plot is irrelevant; it is the bin-to-bin differences that carry information about the relative contributions from different $B \rightarrow X_{c} e \bar{\nu}_{e}$ decays.

\footnotetext{
${ }^{1}$ Recall that all plots of kinematic quantities from the $B \rightarrow D^{0} e \bar{\nu}_{e} X$ control sample have been subtracted for combinatorial background using the $D^{0}$ mass sidebands and for continuum background using the off-peak data.
} 
a)

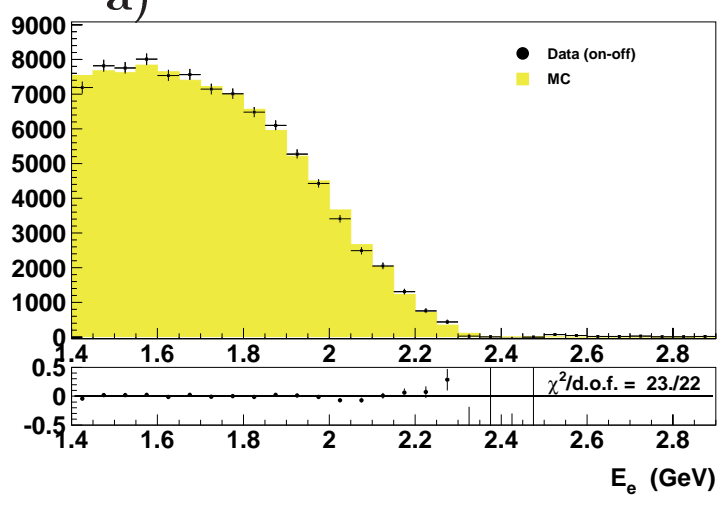

c)

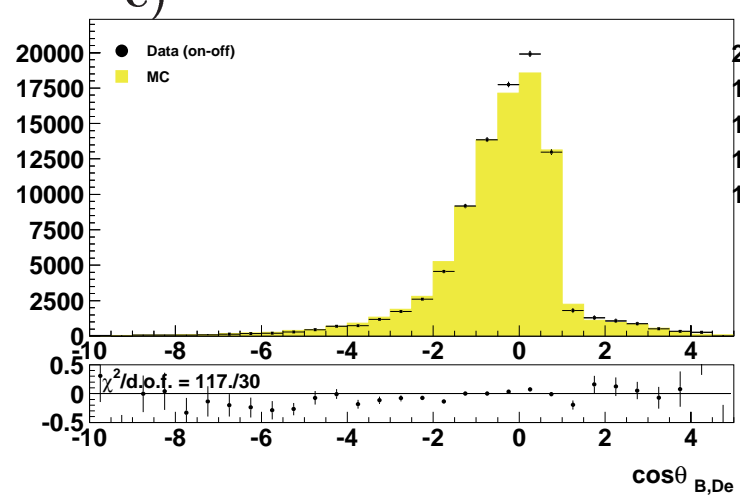

e)

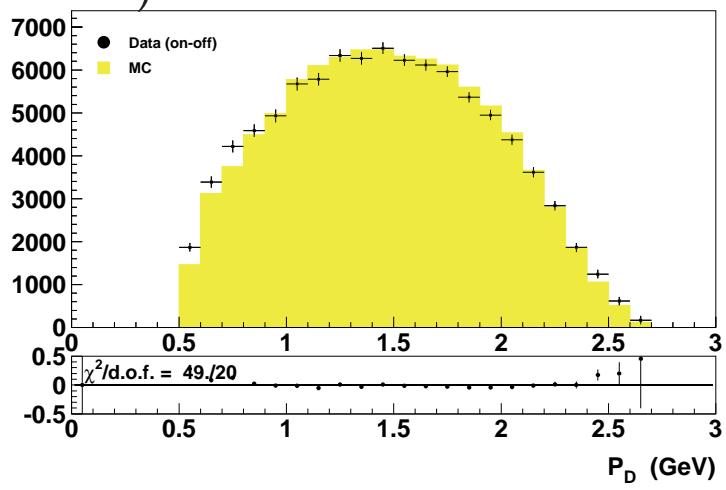

b)

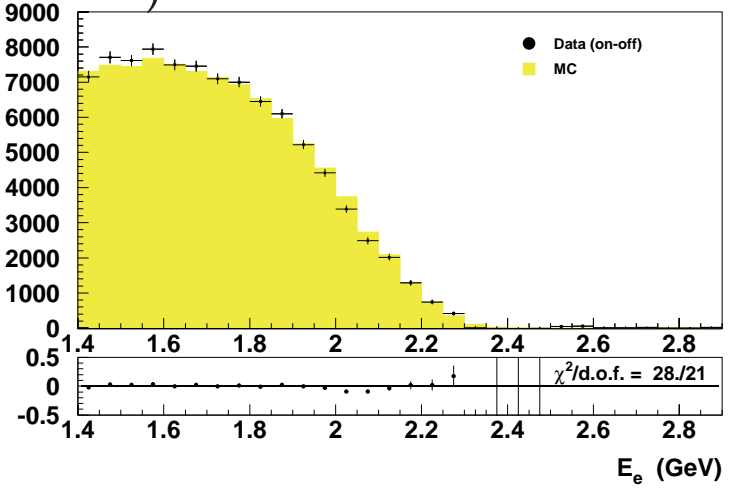

d)

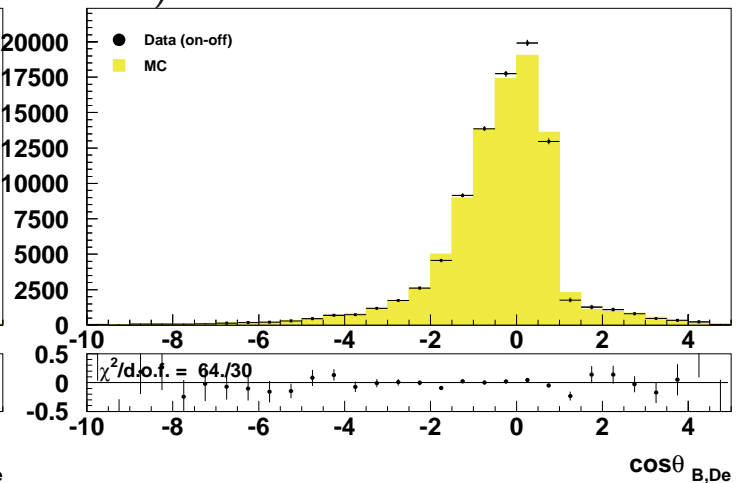

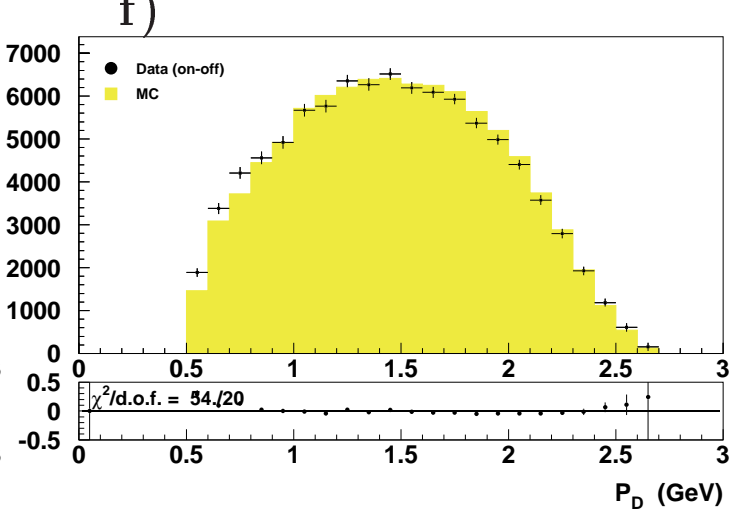

Figure 6.3: Data and Monte Carlo distributions for a) $E_{e}^{*}$, c) $\cos \theta_{B, D^{0} e}$ and e) $p_{D}^{*}$ prior to the $B \rightarrow D^{((*, *)} e \bar{\nu}_{e}$ tuning, and corresponding distributions after applying the tuning (b, $\mathrm{d}, \mathrm{f}$, respectively). Events entering these distributions satisfy the preselection requirements listed in section 5.2. 
a)

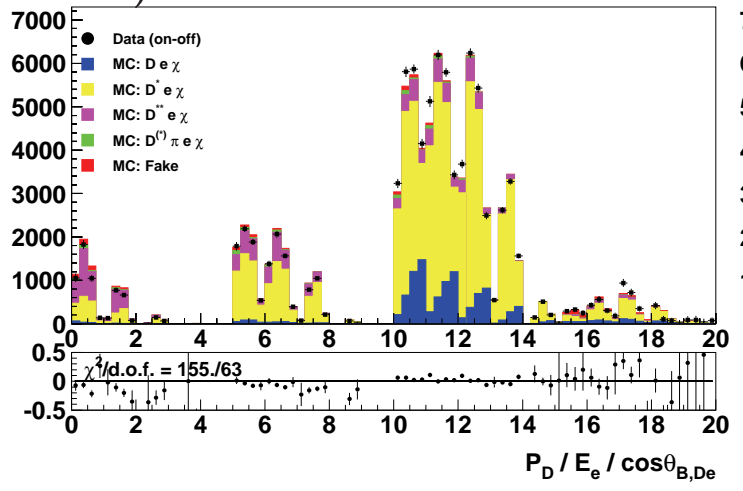

b)

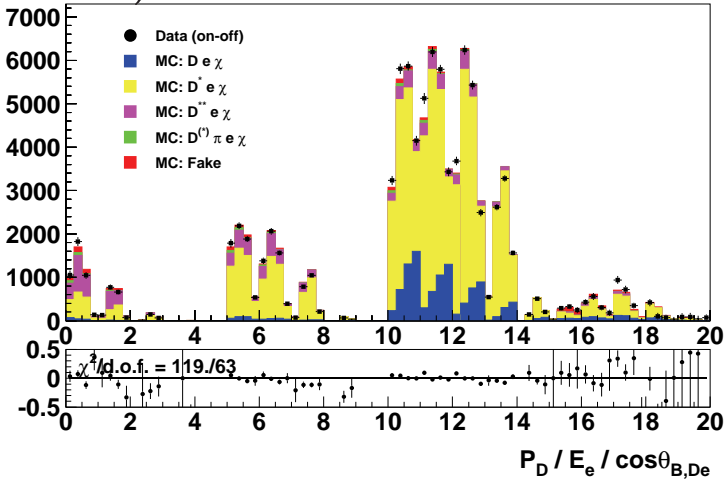

Figure 6.4: Three-dimensional distribution for data and Monte Carlo a) prior to and b) after applying the $B \rightarrow D^{\left({ }^{*, * *}\right)} e \bar{\nu}_{e}$ correction. The text provides a description of the variables shown.

The distribution shown figure 6.4 is fitted to three components: $B \rightarrow D e \bar{\nu}_{e}, B \rightarrow$ $D^{*} e \bar{\nu}_{e}$ and "other". The fit determines a scale factor for each component by minimizing

$$
\chi^{2}\left(x_{1}, x_{2}, x_{3}\right)=\sum_{\text {goodbins }} \frac{\left(N^{\text {data }}-\sum_{i=1}^{3} x_{i} N_{i}^{\mathrm{MC}}\right)^{2}}{N^{\text {data }}+\sigma_{x}^{2}}
$$

where $\sigma_{\mathrm{MC}}^{2}$ is the statistical uncertainty on the nominal MC yields. Only bins for which the MC yield is positive by $>3 \sigma$ are considered. The change in $\sigma_{x}^{2}$ with $x_{i}$ is ignored in the $\chi^{2}$ formula; this is dealt with by iterating the fit twice. Before the fit, a $\chi^{2}$ of 155 for 63 d.o.f. is obtained. The first iteration of the fit returns the following information:

$$
\begin{aligned}
& x_{1}=0.994 \pm 0.045 \\
& x_{2}=0.944 \pm 0.020 \\
& x_{3}=0.717 \pm 0.033 \\
& \chi^{2}=121 \text { for } 63 \text { d.o.f. }
\end{aligned}
$$

A second iteration, in which the 3 scale factors are $0.90,0.91$ and 0.88 , reduces the $\chi^{2}$ to 118 , and a third iteration gives 0.90 for each scale factor and $\chi^{2}=116$, indicating that the fit has converged. The correlations are manageable: -0.73 between $x_{1}$ and $x_{2},-0.59$ between 


\begin{tabular}{|l||c|c|}
\hline Decay type & $B^{0}$ & $B^{+} \rightarrow$ \\
\hline \hline$B \rightarrow D e \bar{\nu}_{e}$ & $2.294 \pm 0.104$ & $2.481 \pm 0.113$ \\
$B \rightarrow D^{*} e \bar{\nu}_{e}$ & $6.024 \pm 0.128$ & $6.520 \pm 0.139$ \\
$B \rightarrow D^{* *} e \bar{\nu}_{e}$ & $1.892 \pm 0.087$ & $2.039 \pm 0.094$ \\
\hline$B \rightarrow X_{c} e \bar{\nu}_{e}$ & $10.21 \pm 0.17$ & $11.04 \pm 0.18$ \\
\hline
\end{tabular}

Table 6.1: Branching fractions for semileptonic $B \rightarrow D^{(*, * *)} e \bar{\nu}_{e}$ decays resulting from the tuning procedure described in the text.

$x_{2}$ and $x_{3}$ and -0.11 between $x_{1}$ and $x_{3}$; none is dangerously close to unity, as would be the case if only information on $E_{e}^{*}$ were used. The scale factors determined in the fit are used to calculate branching fractions under the constraint that the inclusive $B \rightarrow X_{c} e \bar{\nu}_{e}$ branching fractions for $B^{0}$ and $B^{+}$equal the values given in table 4.4:

$$
\begin{aligned}
\mathcal{B}(D e \nu)_{\text {new }} & =x_{1} \mathcal{B}(D e \nu)_{\text {old }} \mathcal{G} \\
\mathcal{B}\left(D^{*} e \nu\right)_{\text {new }} & =x_{2} \mathcal{B}\left(D^{*} e \nu\right)_{\text {old }} \mathcal{G} \\
\mathcal{B}\left(D^{* *} \text { or } D^{(*)} \pi e \nu\right)_{\text {new }} & =x_{3} \mathcal{B}\left(D^{* *} \text { or } D^{(*)} \pi e \nu\right)_{\text {old }} \mathcal{G} \\
\mathcal{G} & =\frac{\sum \mathcal{B}(i)_{\text {old }}}{\sum x_{i} \mathcal{B}(i)_{\text {old }}}=0.90
\end{aligned}
$$

The relative branching fractions of the $B \rightarrow D^{* *} e \bar{\nu}_{e}$ modes and $B \rightarrow D^{(*)} \pi e \bar{\nu}_{e}$ are kept fixed in this procedure; they are varied when assessing systematic uncertainties. The resulting branching fractions listed in table 6.1 .

The fitted values of the $B \rightarrow D^{0} e \bar{\nu}_{e}$ and $B \rightarrow D^{*} e \bar{\nu}_{e}$ branching fractions are substantially higher than measured values for these decays. To understand whether the fit results are reasonable, the agreement between data and the simulation is compared using the nominal and fitted branching fractions on the inclusive $B \rightarrow D^{0} e \bar{\nu}_{e} X$ sample. These distributions are shown in figures 6.5 and 6.6. The agreement between data and the simulation for the reconstructed missing momentum is improved by using the $B \rightarrow D^{0} e \bar{\nu}_{e}$ fit as shown by the smaller $\chi^{2} /$ d.o.f., and the resulting $E_{\text {miss }}^{*}-\left|\vec{P}_{\text {miss }}^{*}\right|$ shows some slight improvement as well. The agreement in terms of the electron momentum is slightly worse and result in $s_{\mathrm{h}}^{\max }$ being overall slightly worse after the correction. The precise determination of 
a)

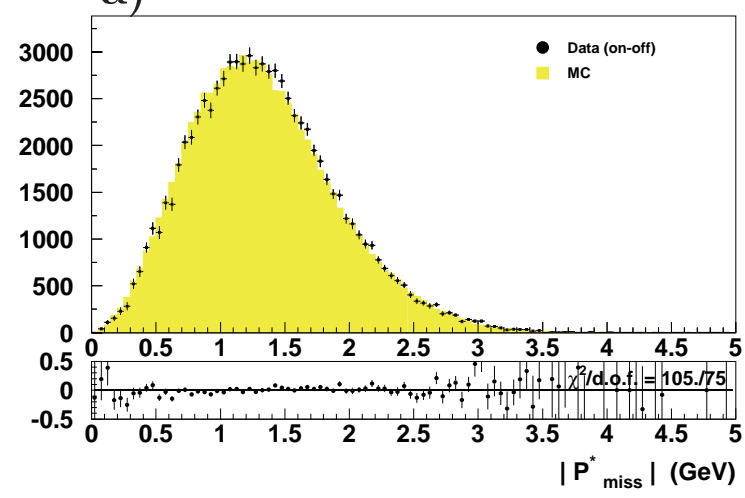

c)

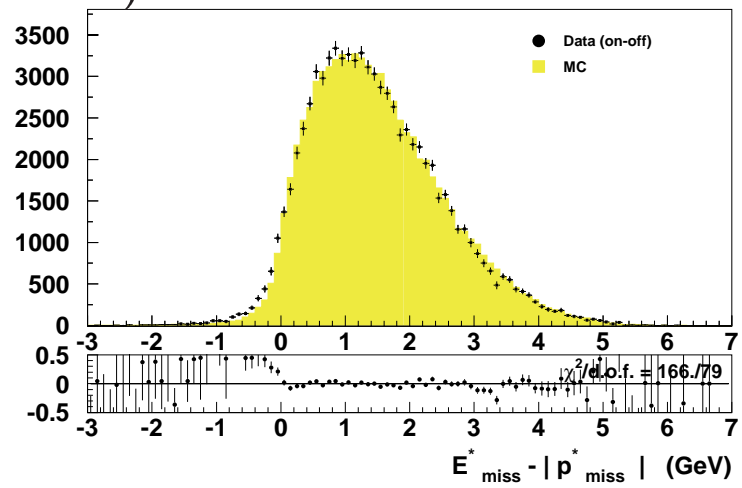

b)

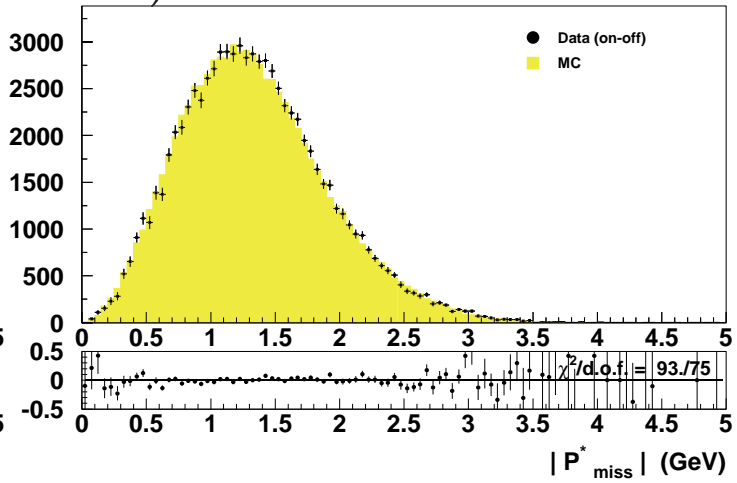

d)

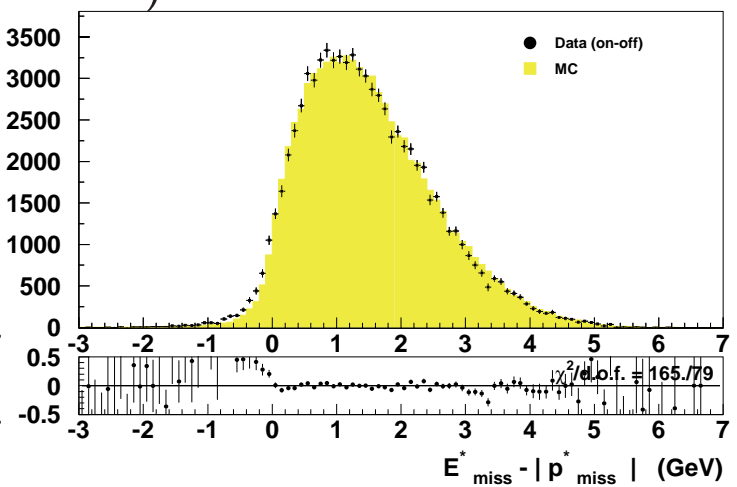

Figure 6.5: Comparison between data and Monte Carlo simulation for a) $\left|\vec{P}_{\text {miss }}^{*}\right|$ and c) $E_{\text {miss }}^{*}-\left|\vec{P}_{\text {miss }}^{*}\right|$ reconstruction with nominal $B \rightarrow D^{\left({ }^{*}, * *\right)} e \bar{\nu}$ branching fractions and corresponding distributions (b and d, respectively) after adjusting the $B \rightarrow D^{\left(\left(^{* * *}\right)\right.} e \bar{\nu}$ contributions. Only events satisfying the requirements listed in section 6.1 enter these distributions.

the overall scale factor, $\mathcal{G}$, is not critical for this analysis due to the sideband normalization used in the signal extraction (see section 7.1).

\subsection{Study of neutrino reconstruction}

The control sample selection makes no requirements on the other $B$ in the event, and can therefore be used to study the impact of the modeling of the other $B$ on the neutrino reconstruction. The neutrino momentum may be evaluated in two different ways. First, 
a)

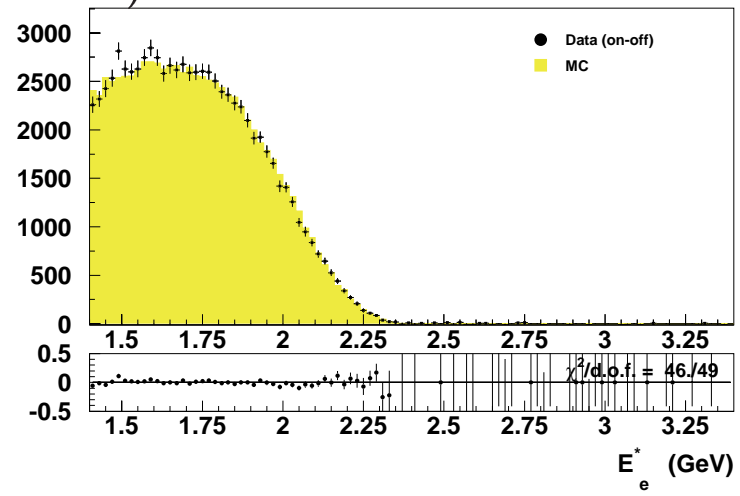

c)

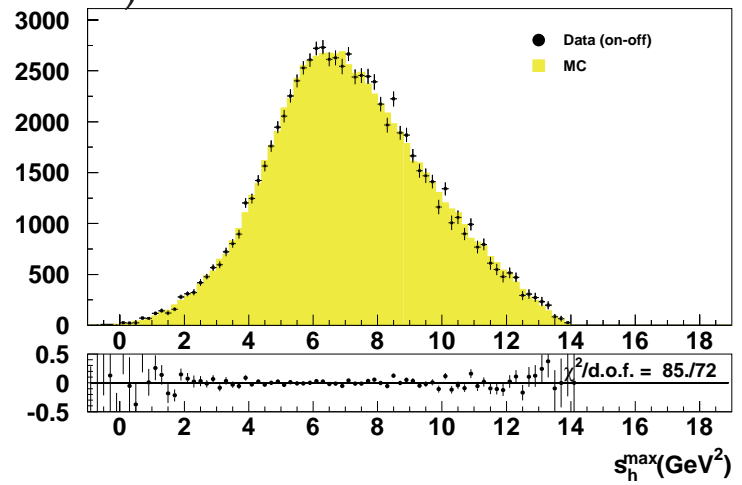

b)

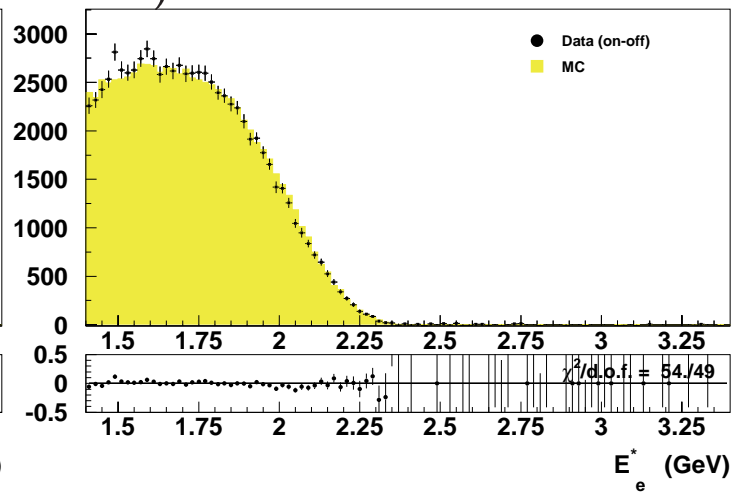

d)

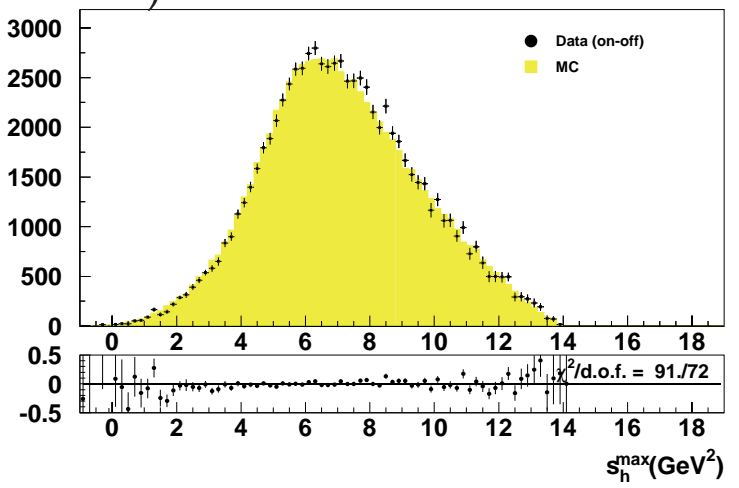

Figure 6.6: Comparison between data and Monte Carlo simulation for a) $E_{e}^{*}$ and c) $s_{\mathrm{h}}^{\max }$ reconstruction with nominal $B \rightarrow D^{\left(\left(^{* * *}\right)\right.} e \bar{\nu}_{e}$ branching fractions and corresponding distributions (b and d, respectively) after adjusting the $B \rightarrow D e \bar{\nu}$ contributions. Only events satisfying the requirements listed in section 6.1 enter these distributions. 
one can consider the neutrino momentum $\vec{P}_{\nu}^{*}$ based on the missing momentum in the event as described in section 5.4. Assuming the only missing particle in the $B \rightarrow D^{0} e \bar{\nu}_{e} X$ decay is the neutrino, one can also use the energies of the $D$ and $e$ to infer the momentum of the neutrino:

$$
E_{\nu}^{D^{0} e}=E_{B}^{*}-E_{D}^{*}-E_{e}^{*},
$$

where the above estimators are computed in the center-of-mass frame. By construction, most $D^{*}$ decays into $D^{0}$ feed into the control sample. The small $q$-value in the decay means that the 4-momentum of the $D^{*}$ can be estimated based on the $D^{0}$ as

$$
p_{D^{*}}^{*} \simeq p_{D}^{*} \frac{m_{D^{*}}}{m_{D}}
$$

For each $D^{0} e \bar{\nu}_{e}$ candidate, a compatibility test is performed by computing $\cos \theta_{B, D^{*} e}$, where the $D^{*} e$ combination is formed using the 4-momentum given above. If $\cos \theta_{B, D^{*} e}<1$, then the $D^{*}$ hypothesis is taken and $E_{\nu}^{D^{*} e}$ is computed as

$$
E_{\nu}^{D^{*} e}=E_{B}^{*}-E_{e}^{*}-E_{D^{0}}^{*} \cdot \frac{m_{D^{*}}}{m_{D^{0}}}
$$

otherwise,

$$
E_{\nu}^{D^{0} e}=E_{B}^{*}-E_{e}^{*}-E_{D^{0}}^{*}
$$

In figure 6.7 the resolution for $E_{\nu}^{*}$ from Monte Carlo is plotted using the above definitions. Note the significantly better resolution when using $E_{\nu}^{D^{(*)} e}$ to estimate the neutrino momentum. As a result, the width of the distribution of the difference between these estimators is dominated by the $\left|\vec{P}_{\nu}^{*}\right|$ resolution, allowing a comparison between data and Monte Carlo for this "pseudo-resolution" quantity.

Figure 6.8 shows comparison between data and Monte Carlo for the missing momentum and energy, and for the two neutrino estimates $\left|\vec{P}_{\nu}^{*}\right|$ and $E_{\text {miss }}^{D^{(*)}}$. Reasonable agreement is achieved for all of the quantities. The resulting pseudo-resolutions $\left|\vec{P}_{\text {miss }}^{*}\right|-E_{\text {miss }}^{D^{(*)} e}$ and $\left|\vec{P}_{\nu}^{*}\right|-E_{\text {miss }}^{D^{(*)} e}$ are also shown in figure 6.8. In both cases, a good agreement is found between data and MC before and after applying the refined selection but the neutrino correction offers a much better estimate of the true neutrino momentum. The average (r.m.s.) 
a)

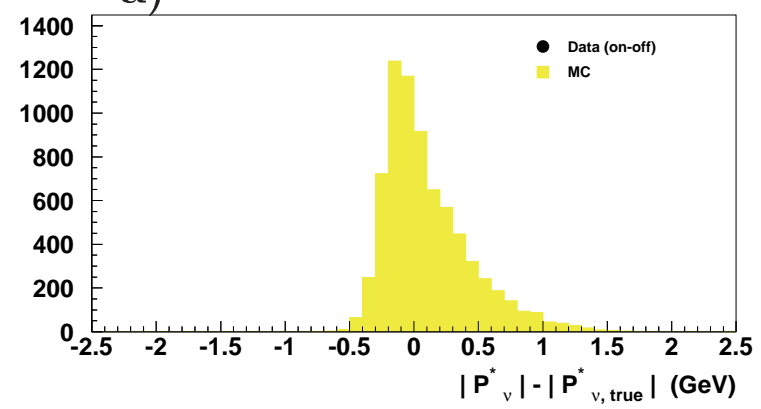

b)

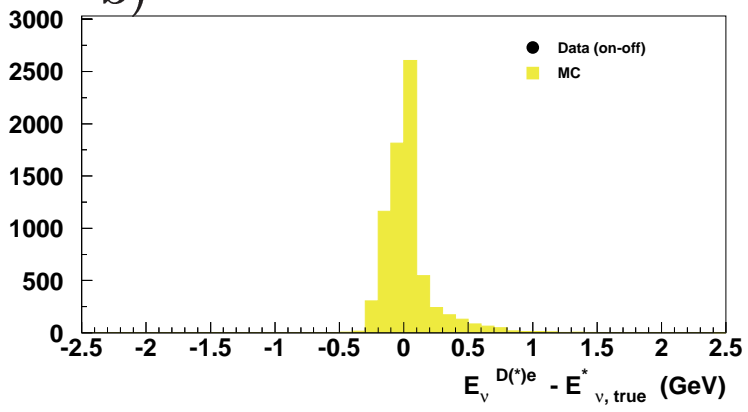

Figure 6.7: Resolution plots using the simulation for the two neutrino estimators, a) $\left|\vec{P}_{\nu}^{*}\right|-$ $\left|\vec{P}_{\nu, \text { true }}\right|$ and b) $E_{\nu}^{D^{(*)} e}-E_{\nu, \text { true }}$ for the $D^{0} e \bar{\nu}_{e} X$ control sample for events satisfying the refined selection defined in section 5.5 (but not $s_{h}^{\max }$ ).

$\left|\vec{P}_{\nu}^{*}\right|-E_{\text {miss }}^{D^{(*)} e}$ for events satisfying the refined selection is $0.101 \mathrm{GeV}(0.472 \mathrm{GeV})$ for data and $0.101 \mathrm{GeV}(0.474 \mathrm{GeV})$ for simulated events. In comparison, the $\left|\vec{P}_{\text {miss }}^{*}\right|-E_{\text {miss }}^{D^{(*)} e}$ average (r.m.s.) is about $0.700 \mathrm{GeV}(0.40 \mathrm{GeV})$ for events satisfying the refined selection for both data and Monte Carlo. The $\left|\vec{P}_{\nu}^{*}\right|$ is therefore a better estimate of the true neutrino momentum, as designed. The good agreement between data and the simulation in the $\left|\vec{P}_{\nu}^{*}\right|-E_{\text {miss }}^{D^{(*)}}$ also demonstrates that the simulation of the other $B$ in the event is adequate.

\subsection{Additional tests}

The validation of the neutrino resolution on the $B \rightarrow D^{0} e \bar{\nu}_{e} X$ control sample, where the $D^{0}$ from the semileptonic $B$ decay is fully reconstructed, is not nearly as sensitive to missing energy from $D$ decays as is the signal sample, in which the $D$ and $\nu$ both recoil against the high energy signal electron and are more likely to survive the $E_{\text {miss }}^{*}-\left|\vec{P}_{\text {miss }}^{*}\right|$ cut. To further investigate the modeling of $D$ decays, the distributions of electrons and $K_{S}^{0}$ in the hemisphere opposite to the signal electron are considered. The electrons are associated with additional neutrinos, and the $K_{S}^{0}$ are studied as they should have the same rate and distribution as the potentially problematic $K_{L}^{0}$. The relevant distributions are shown in figure 6.9. The data and Monte Carlo are in each case in reasonable agreement using both 
a)

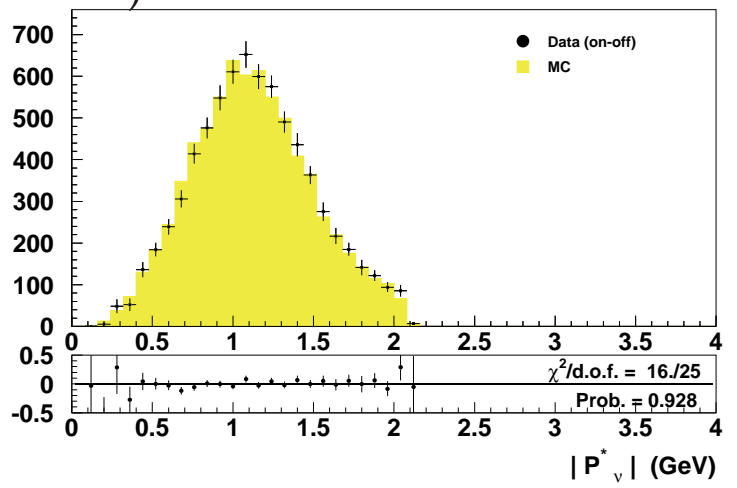

C)

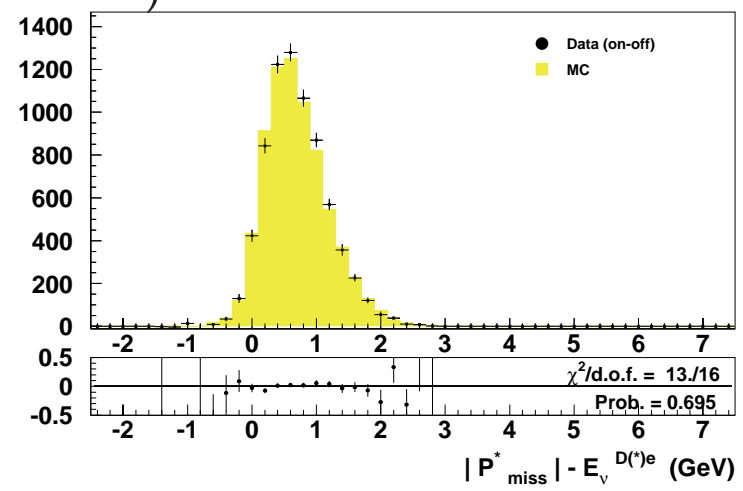

b)

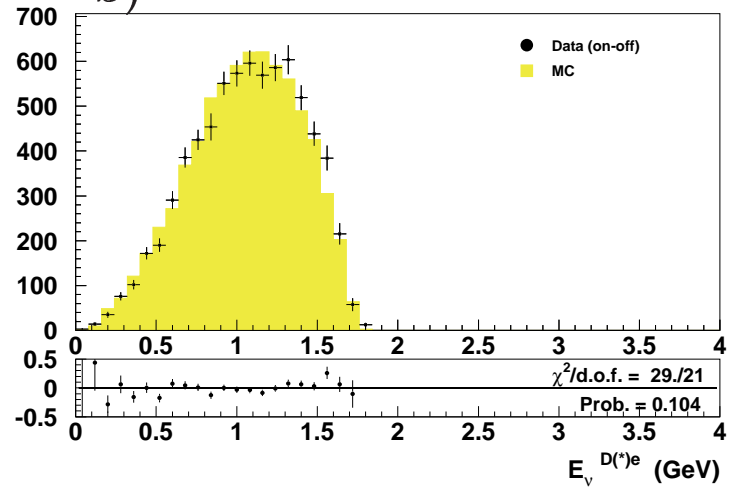

d)

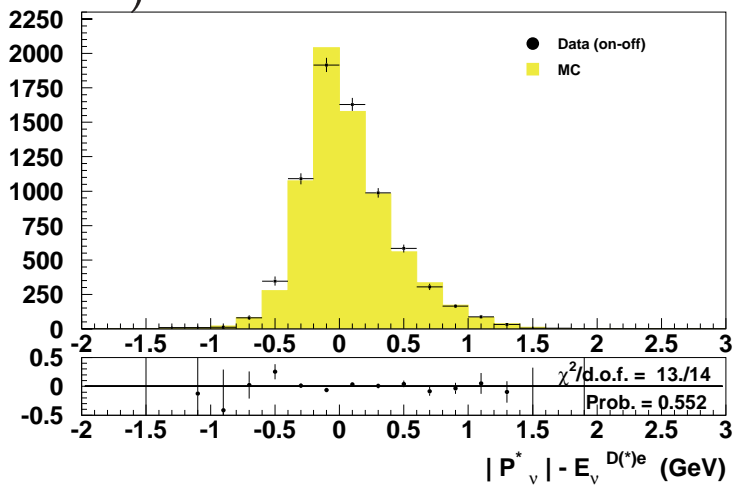

Figure 6.8: Data and Monte Carlo distributions using the $D^{0} e \bar{\nu}_{e} X$ control sample for a) $\left|\vec{P}_{\nu}^{*}\right|$, b) $E_{\nu}^{D^{(*)} e}$, c) $\left|\vec{P}_{\text {miss }}^{*}\right|-E_{\text {miss }}^{D^{(*)} e}$ and d) $\left|\vec{P}_{\nu}^{*}\right|-E_{\text {miss }}^{D^{(*)} e}$ for events satisfying the requirements outlined in section 6.1 and the refined event selection of section 5.5 (excluding $s_{\mathrm{h}}^{\max }$ ). 
the signal and $B \rightarrow D^{0} e \bar{\nu}_{e} X$ control samples.

As expected, more opposite-sign electrons than like-sign electrons are observed since there is either a semileptonic $B$ decay followed by a semileptonic charm decay, or there is a hadronic $B$ decay, $b \rightarrow c \bar{c} q$ where both charm quarks decay semileptonically or there are semileptonic decays of both $B$ mesons (the opposite lepton charge correlation is diluted in case of neutral $B$-decays). Combinations leading to like-sign electrons are possible with other configurations but less frequent. However, they preferably occur in background rather than in $B \rightarrow X_{u} e \bar{\nu}_{e}$ since in this case secondary electrons from the same side are highly suppressed.

These comparisons do not address possible uncertainties due to form factors in $D \rightarrow K^{(*)} e \bar{\nu}_{e}$ decay or the energy deposition of $K_{L}^{0}$ in the EMC, which will be evaluated in chaper 7. They also have contributions from sources other than the $D$ from the semileptonic $B$ decay that produced the high energy electron, i.e. their sensitivity to the modeling of $D$ decays is somewhat limited.

\subsection{Adjusting Monte Carlo efficiencies}

The event selection requirements listed in section 5.5 render the efficiency predictions sensitive to the details of the Monte Carlo simulation. To reduce this sensitivity, the ratio of the $B \rightarrow D^{0} e \bar{\nu}_{e} X$ sample efficiencies for data and Monte Carlo is used. In order to compute these efficiencies, the yields are determined by first counting the number of entries in the signal region, defined as within $2.5 \sigma(\sim 15 \mathrm{MeV})$ of the fitted $D^{0}$ mass peak after subtracting from them the number of combinatorial background events estimated from the sideband regions, defined as within $3.75 \sigma$ to $6.25 \sigma(22.5$ to $37.5 \mathrm{MeV})$ of the fitted $D^{0}$ mass. As such, the fit consists of a Gaussian distribution plus a constant:

$$
f(m)=P_{1} \exp \left(\frac{m-P_{2}}{\sqrt{2} P_{3}}\right)^{2}+P_{4},
$$

where the $P_{i}$ 's are the parameters being fitted for. Figure 6.10 displays the $D^{0}$ mass spectra for both data and Monte Carlo after applying the $D^{0}$ requirements and the refined event 
a)

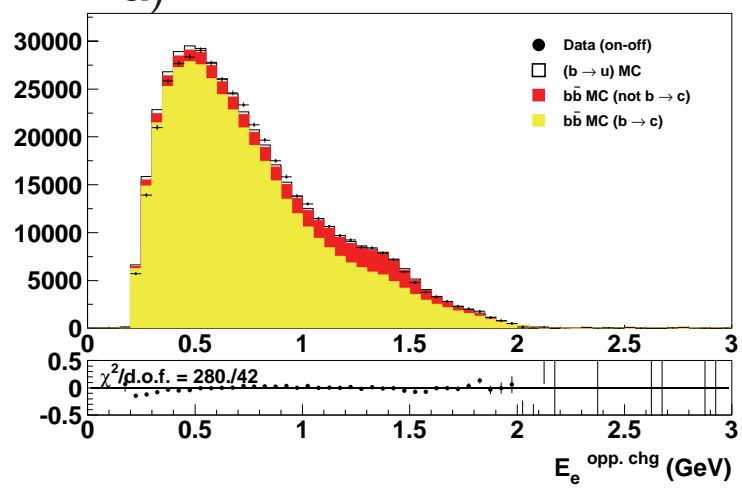

c)

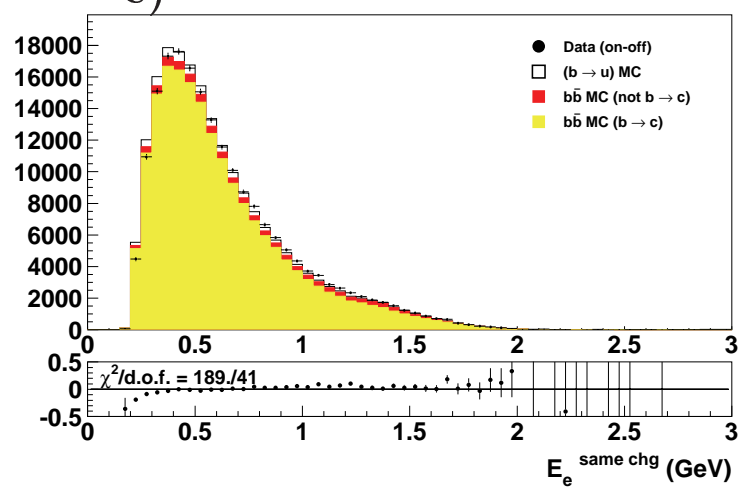

e)

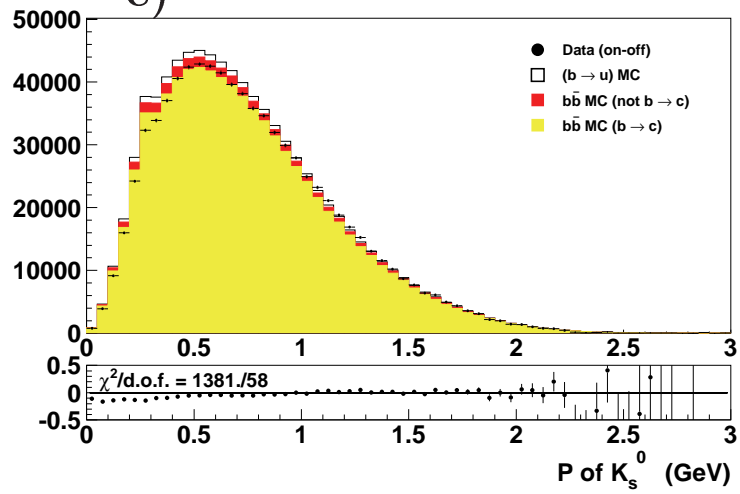

b)

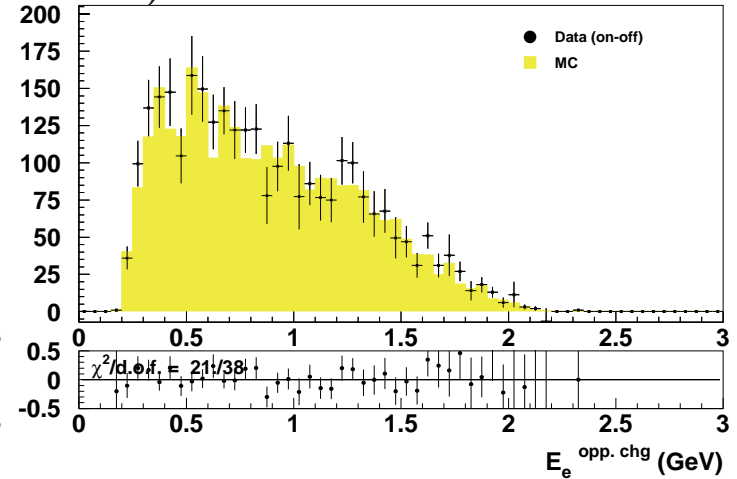

d)

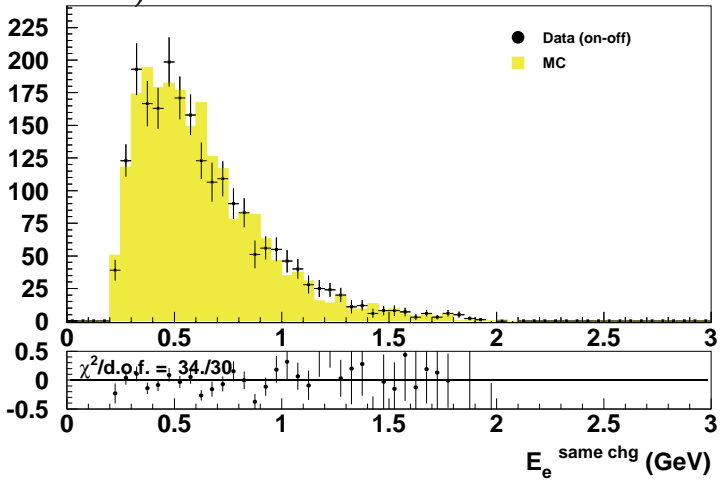

f)

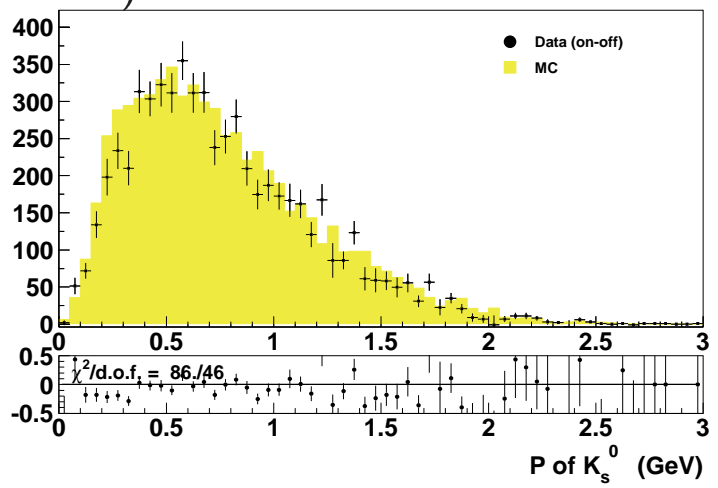

Figure 6.9: Data and Monte Carlo comparison for a) opposite-sign and c) same-sign electron energy distributions, and c) $K_{S}^{0}$ momentum for particles in the hemisphere opposite the signal electron for the signal sample and $B \rightarrow D^{0} e \bar{\nu}_{e} X$ control sample (b, $\mathrm{d}$, and $\mathrm{f}$, respectively). 
a)

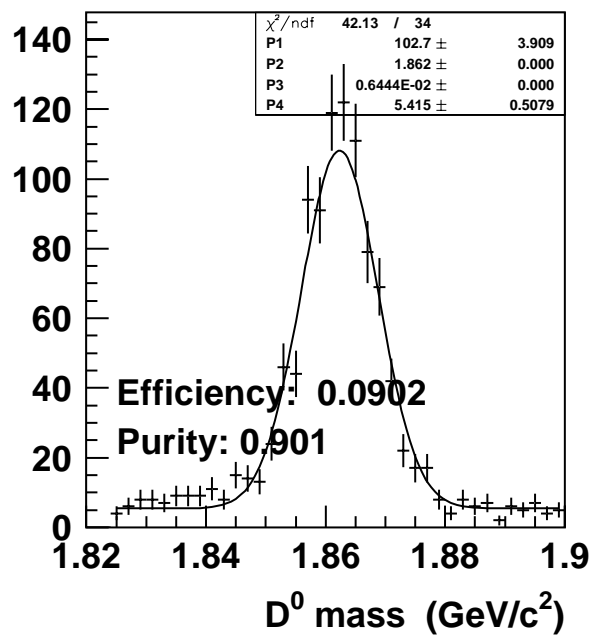

b)

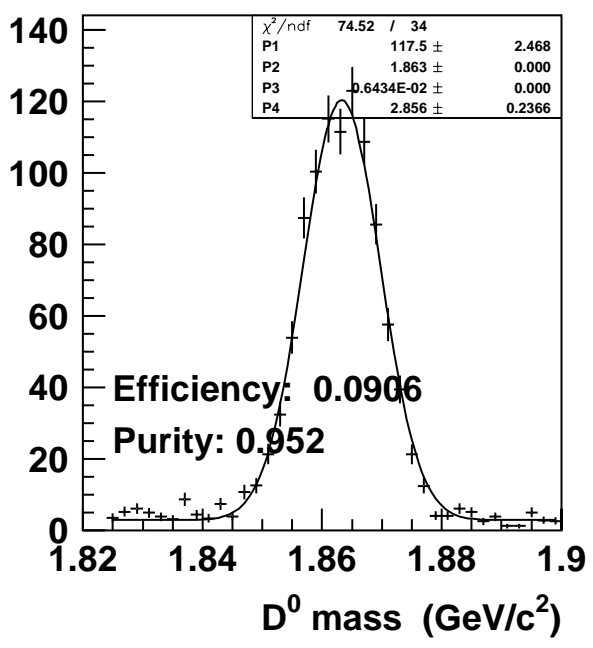

Figure 6.10: $D^{0}$ Mass spectrum for a) data and b) Monte Carlo after applying the selection cuts (except $\left.s_{\mathrm{h}}^{\max }\right)$. The signal region is defined as within $2.5 \sigma(\sim 15 \mathrm{MeV})$ of the fitted $D^{0}$ mass peak, whereas continuum is estimated from the sideband regions taken within $3.75 \sigma$ to $6.25 \sigma(22.5 \mathrm{MeV}$ to $37.5 \mathrm{MeV})$ of the fitted $D^{0}$ mass peak.

selection (section 5.5) except on $s_{\mathrm{h}}^{\max }$. The fits do not attempt to account for peaking backgrounds, e.g. from $B \rightarrow D^{(*)} \bar{D}^{(*)}$ where one of the $D$ mesons decays semileptonically and the other to $\mathrm{K}^{-} \pi^{+}$, as their contribution is small and is relevant only to the extent that they differ in data and Monte Carlo. There is a small $3 \mathrm{MeV}(2 \mathrm{MeV})$ difference between the fitted mean in data (Monte Carlo) and the average $D^{0}$ mass measurement.

The yields after each selection requirement can be used to form relative and cumulative efficiencies. These are listed in table 6.2 , where the sample satisfying the $B \rightarrow D^{0} e \bar{\nu}_{e} X$ selection requirements defined the denominator in computing the efficiencies. Notice that comparable efficiencies are obtained for data and the simulation. These are used to adjust the Monte Carlo prediction for the $B \rightarrow X_{u} \ell \bar{\nu}_{\ell}$ signal efficiency according to

$$
\epsilon_{b \rightarrow u}^{\mathrm{data}}=\epsilon_{b \rightarrow u}^{\mathrm{MC}}\left(\frac{\epsilon_{D^{0} e \overline{\nu_{e}}}^{\mathrm{data}}}{\epsilon_{D^{0} e \bar{\nu}_{e}}^{\mathrm{MC}}}\right),
$$

where the above efficiencies are taken from table 6.2 , and correspond to the cumulative efficiencies before applying the $s_{\mathrm{h}}^{\max }$ requirement. The latter selection criterion is omitted 


\begin{tabular}{|l|c|c|c|c|}
\hline Applied cut & \multicolumn{2}{|c|}{ Data } & \multicolumn{2}{c|}{ MC } \\
\hline \hline$D^{0} \ell \nu$ & \multicolumn{2}{|c|}{$8785 \pm 218$} & \multicolumn{2}{c|}{$10509 \pm 106$} \\
\hline Requirements & $\epsilon$ & cumul. $\epsilon$ & $\epsilon$ & cumul. $\epsilon$ \\
\hline \hline Bhabha veto & $0.9954 \pm 0.0201$ & $0.9954 \pm 0.0201$ & $0.9946 \pm 0.0089$ & $0.9946 \pm 0.0089$ \\
$N_{\text {leptons }}$ & $0.7869 \pm 0.0159$ & $0.7834 \pm 0.0158$ & $0.7731 \pm 0.0068$ & $0.7692 \pm 0.0068$ \\
$P_{\text {miss }}^{*}$ & $0.9811 \pm 0.0198$ & $0.7720 \pm 0.0156$ & $0.9820 \pm 0.0088$ & $0.7560 \pm 0.0067$ \\
$E_{\text {miss }}^{*}-\left|P_{\text {miss }}^{*}\right|$ & $0.2914 \pm 0.0086$ & $0.2313 \pm 0.0075$ & $0.2910 \pm 0.0037$ & $0.2217 \pm 0.0034$ \\
$\cos \theta_{\text {miss }}^{*}$ & $0.7847 \pm 0.0157$ & $0.1713 \pm 0.0064$ & $0.7860 \pm 0.0069$ & $0.1666 \pm 0.0027$ \\
$\cos \theta_{\vec{p} l}^{*} \vec{T}$ & $0.5464 \pm 0.0118$ & $0.0902 \pm 0.0039$ & $0.5484 \pm 0.0053$ & $0.0906 \pm 0.0019$ \\
\hline
\end{tabular}

Table 6.2: Marginal and cumulative efficiency for the $B \rightarrow D^{0} e \bar{\nu}_{e} X$ control sample. The first row provides the data and Monte Carlo yields for events satisfying the $D^{0} e \bar{\nu}_{e}$ selection outline in section 6.1: selection efficiencies are calculated with respect to these yields. Note that $\epsilon_{D^{0} e \bar{\nu}_{e}}^{\mathrm{data}}$ and $\epsilon_{D^{0} e \bar{\nu}_{e}}^{\mathrm{MC}}$ correspond to the cumulative efficiencies in the last line.

since it is designed to remove semileptonic $B \rightarrow X_{c} \ell \bar{\nu}_{\ell}$ decays and would suppress our control sample. The efficiency correction factor is

$$
(902 \pm 39) /(906 \pm 19)=(0.996 \pm 0.048)
$$

While this correction factor is unity within error, applying it allows the efficiency to be far less sensitive to changes in the simulation. When the systematic studies are performed this correction factor is also taken into account; without it some effects from the systematic uncertainties would be much larger as this factor is not unity when considering some systematic variations as will be demonstrated in chapter 7 .

\subsection{Comparison between data and simulation}

Figures 6.11 to 6.14 diplay various kinematical distributions for events satisfying the $D^{0} e \bar{\nu}_{e}$ selection and events satisfying the refined selection listed in section 5.5; all of the criteria listed therein were applied in producing the latter plots except when a cut corresponded to the variable plotted, in which case the selected region is denoted by an arrow. The selection excludes the requirements on $E_{e}^{*}$ and $s_{\mathrm{h}}^{\max }$ in order to have a reasonable rate. The distributions of events with $K^{-} \pi^{+}$invariant masses in the $D^{0}$ mass sideband regions are 
subtracted from the corresponding distributions of events within the $D^{0}$ mass window. ${ }^{2}$

As shown in figure 6.11 , the event requirements do not introduce any discrepancy between data and the simulation in terms of the event multiplicity and energy reconstruction when comparing with the results shown in figures 5.1 and 5.2. The particle selection also results in a net improvement of the $\chi^{2}$ probability. Note that the latter was computed only in the selected region of the phase-space, and the associated $\chi^{2}$ probability is also displayed. Similarly, reasonable agreement between the data and Monte Carlo simulation is achieved for the neutrino reconstruction in terms of the missing momentum and energy, and $E_{\text {miss }}^{*}-\left|\vec{P}_{\text {miss }}^{*}\right|$ and $\cos \theta_{\text {miss }}^{*}$, as presented in figures 6.12 and 6.13 , respectively. Figure 6.14 displays the $q^{2}$, electron energy and $s_{\mathrm{h}}^{\max }$ distributions. Overall, a reasonable agreement is achieved between data and simulation within the selected phase-space regions, with $\chi^{2}$ probabilities of $13 \%$ or better even though the (larger) systematic uncertainties were not included in calculating the $\chi^{2}$. Therefore, the simulation appears adequate for both the control sample and the other $B$ in the event and the analysis may proceed to the branching fraction measurement and the evaluation of the systematics.

\footnotetext{
${ }^{2}$ The Monte Carlo yield was scaled to the area of the data. This scale factor (0.9) was discussed in section 6.2 .
} 
a)

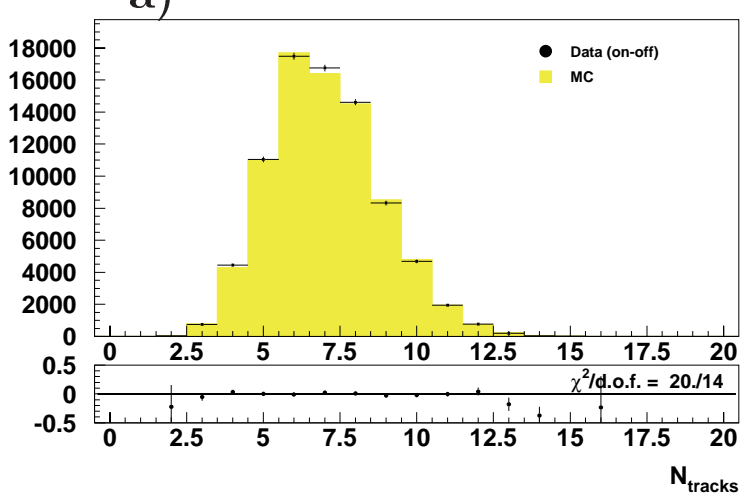

c)

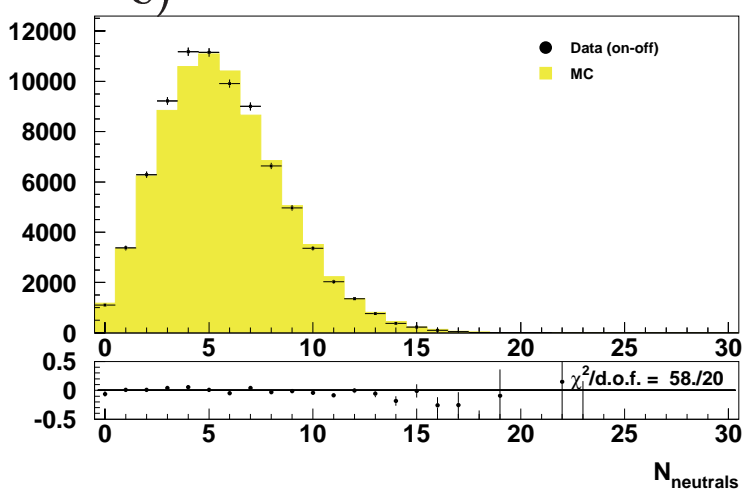

b)

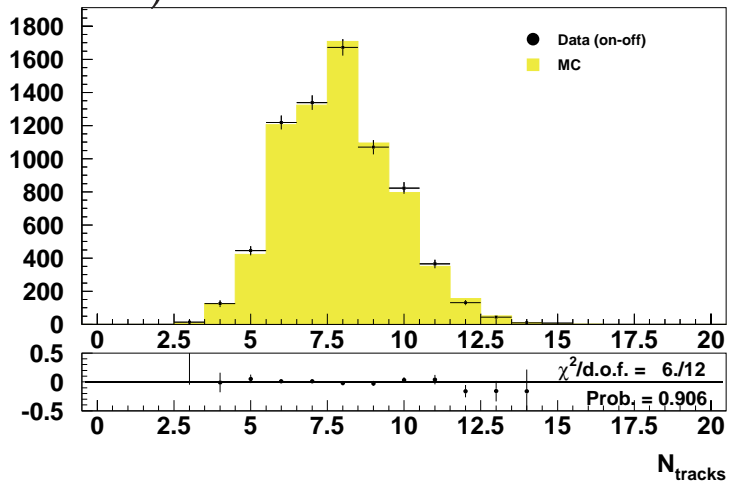

d)

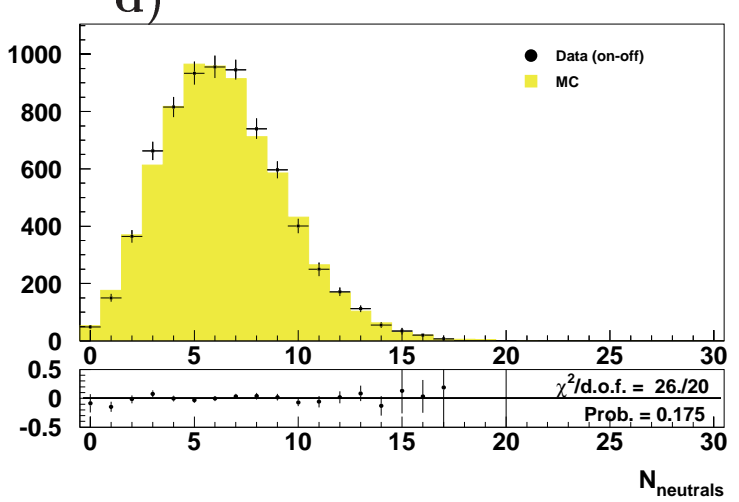

Figure 6.11: Data and Monte Carlo distributions for a) charged track and c) neutral cluster multiplicity for events satisfying the $D^{0} \ell \bar{\nu}_{\ell}$ selection, and the corresponding distributions (b and d) for events satisfying the refined selection described in section 5.5 (excluding $s_{\mathrm{h}}^{\max }$ ). The plots follow the same conventions as those of figure 5.1. 
a)

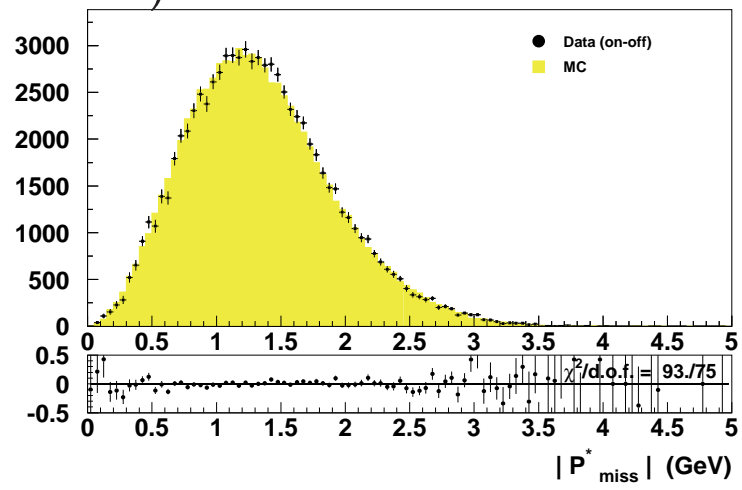

c)

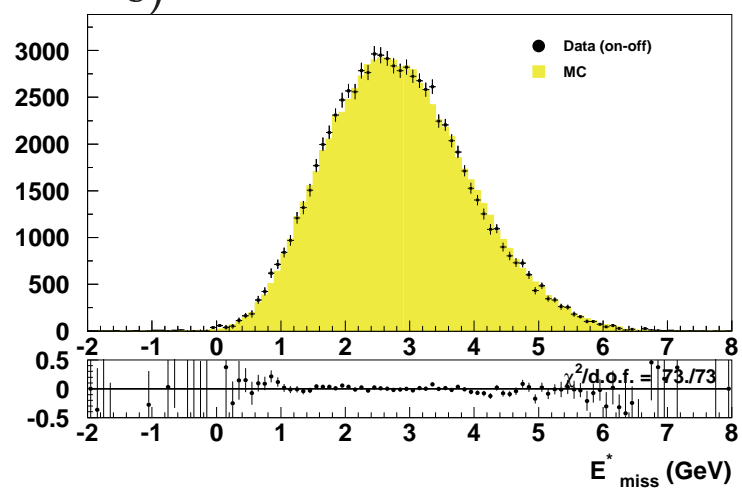

e)

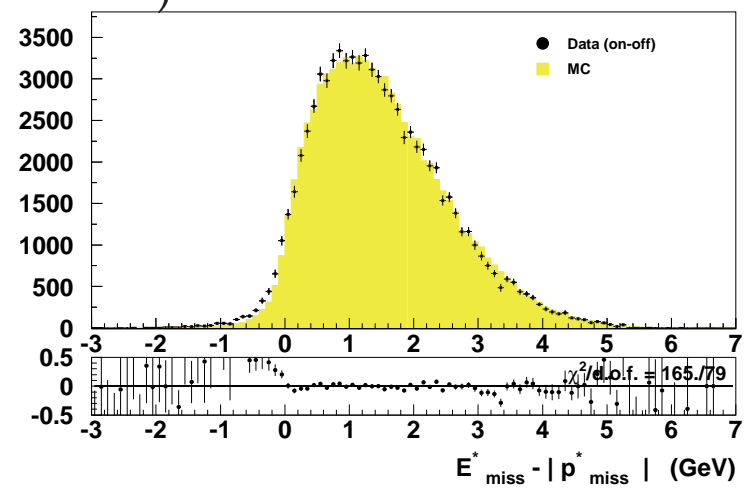

b)

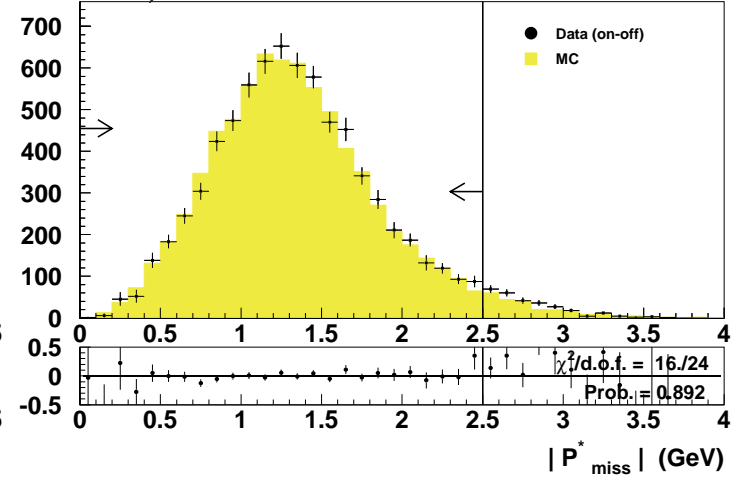

d)

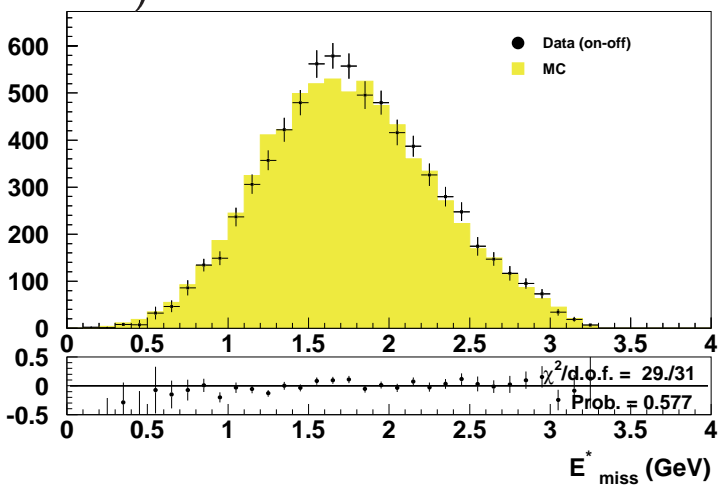

Figure 6.12: Data and Monte Carlo distributions for a) $\left|\vec{P}_{\mathrm{miss}}^{*}\right|$, c) $E_{\mathrm{miss}}^{*}$ and e) $E_{\text {miss }}^{*}-\left|\vec{P}_{\mathrm{miss}}^{*}\right|$ for events satisfying the $D^{0} \ell \bar{\nu}_{\ell}$ selection, and the corresponding distributions $(\mathrm{c}, \mathrm{d}$ and $\mathrm{f}$, respectively) for events satisfying the refined selection described in section 5.5 (excluding $\left.s_{\mathrm{h}}^{\max }\right)$. The plots follow the same conventions as those of figure 5.1. 
a)

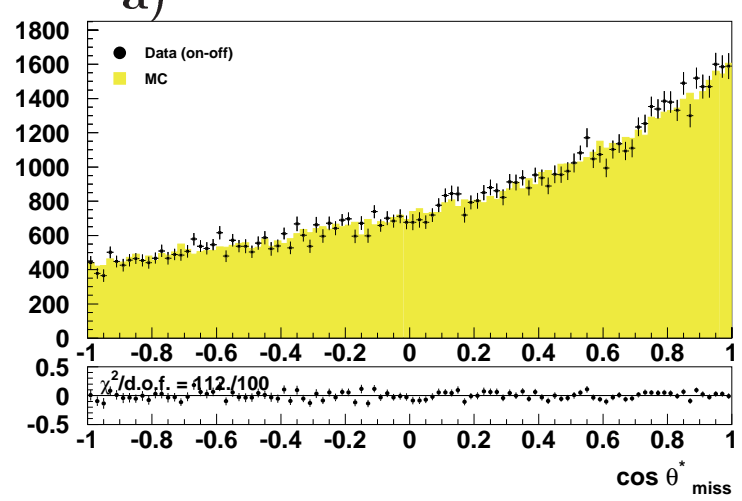

c)

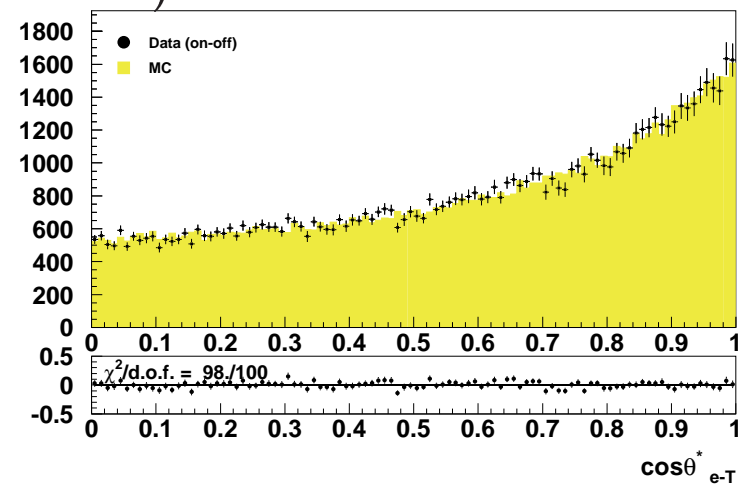

b)

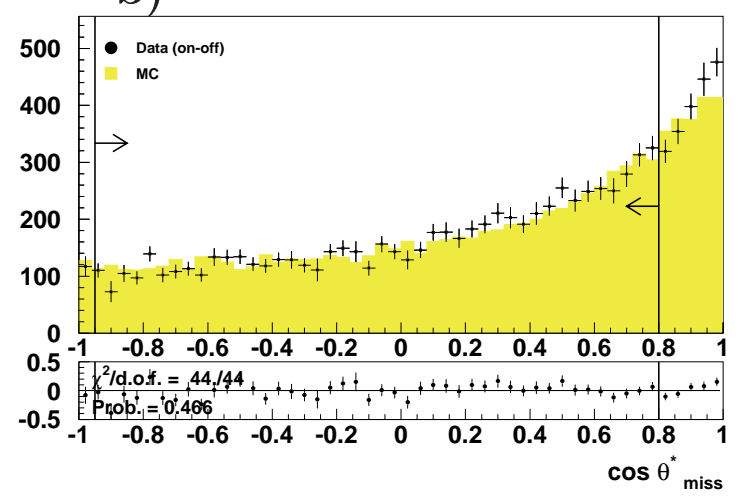

d)

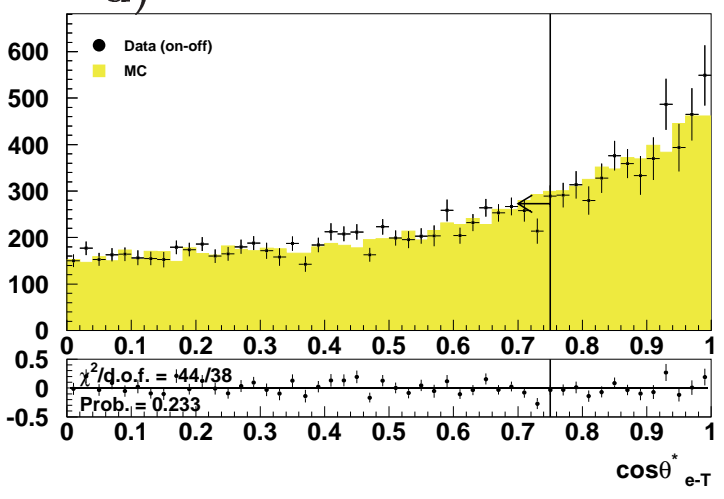

Figure 6.13: Data and Monte Carlo distributions for a) $\cos \theta_{\text {miss }}^{*}$ and b) $\cos \theta_{\vec{e} \cdot \vec{T}}^{*}$ for events satisfying the $D^{0} \ell \bar{\nu}_{\ell}$ selection, and the corresponding distributions (c and d) for events satisfying the refined selection described in section 5.5 (excluding $s_{\mathrm{h}}^{\max }$ ). The plots follow the same conventions as those of figure 5.1. 
a)

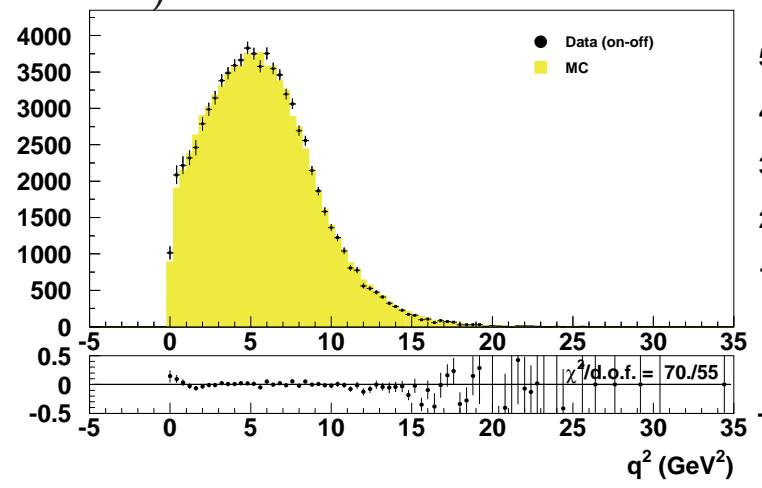

c)

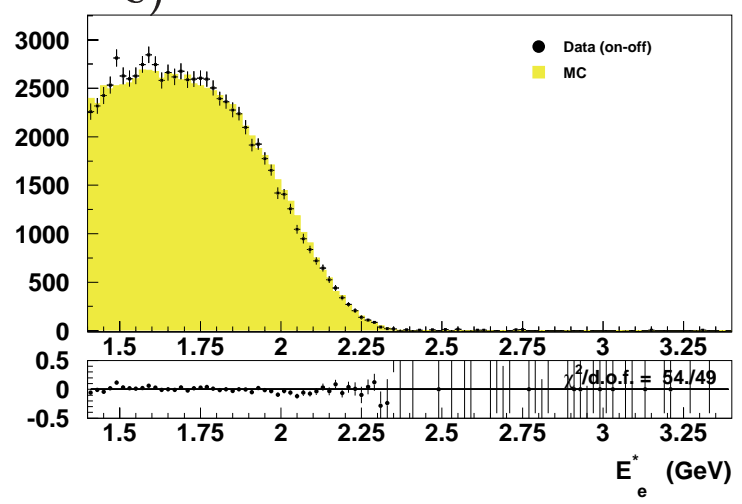

e)

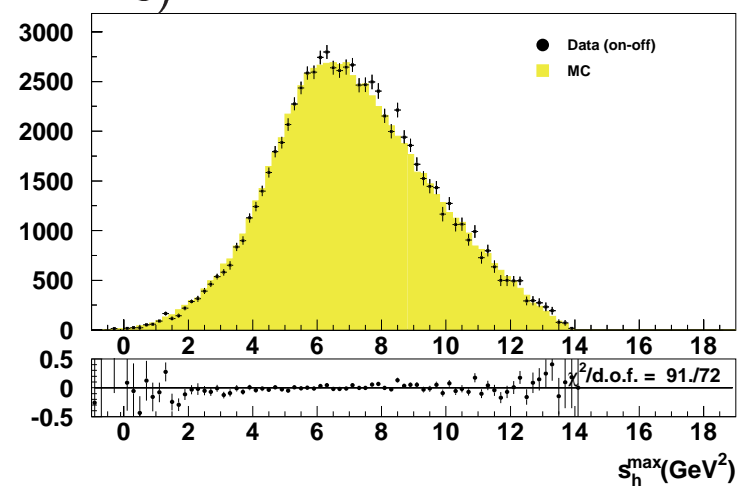

b)

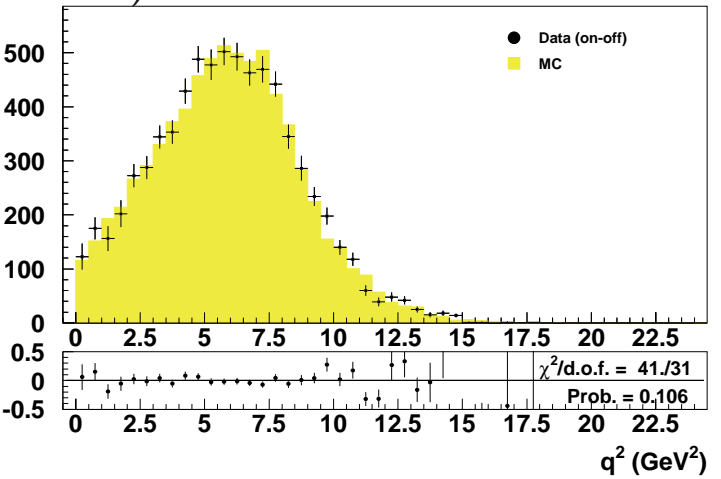

d)

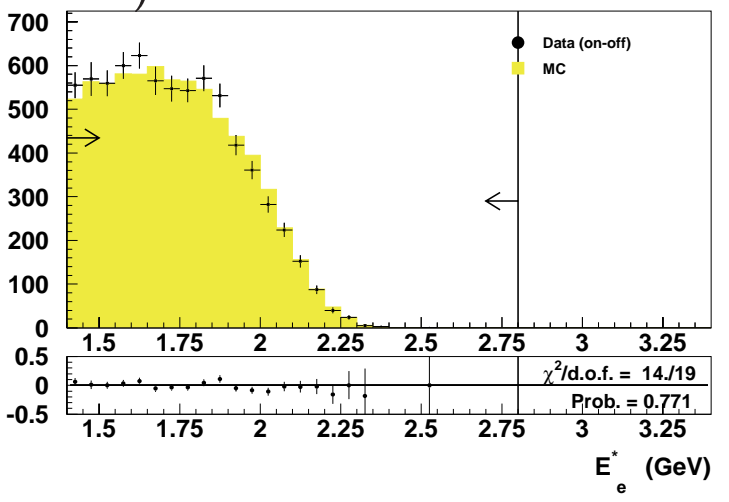

f)

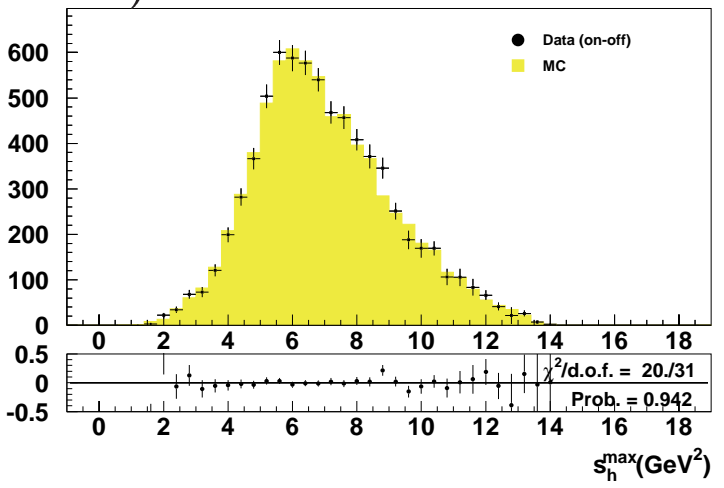

Figure 6.14: Data and Monte Carlo distributions for a) $q^{2}$, c) $E_{e}^{*}$ and e) $s_{\mathrm{h}}^{\max }$ for events satisfying the $D^{0} \ell \bar{\nu}_{\ell}$ selection, and the corresponding distributions (b, d and f, respectively) for events satisfying the refined selection described in section 5.5 (excluding $s_{\mathrm{h}}^{\max }$ ). The plots follow the same conventions as those of figure 5.1. 


\section{Chapter 7}

\section{Systematics Studies}

Errare humanum est

\subsection{Signal extraction}

No major discrepancies were found between data and Monte Carlo simulations in terms of the signal sample (chapter 5) and $B \rightarrow D^{0} e \bar{\nu}_{e} X$ control sample (chapter 6). Hence, the simulation of background events and the other $B$ decay in the event appears to be adequate and one may proceed to the next stage of the analysis. In order to extract the signal $\mathcal{B}\left(B \rightarrow X_{u} e \bar{\nu}_{e}\right)$, the yield for events satisfying the event selection outlined in section 5.5 is extracted for both data and the simulation. The $B \rightarrow X_{u} e \bar{\nu}_{e}$ yield is computed as the number of candidates in data within the signal region minus the number of background events estimated from the Monte Carlo simulation. Equation 5.1 may then be rewritten as

$$
\mathcal{B}_{u}^{\mathrm{SL}}=\frac{N_{\mathrm{cand}}^{\mathrm{data}}-N_{\mathrm{bkg}}}{2 N_{B \bar{B}} \epsilon_{u}}
$$

where $N_{B \bar{B}}=88.35 \times 10^{6}$ is the number of $\Upsilon(4 S) \rightarrow B \bar{B}$ decays analyzed. Relying solely on the Monte Carlo for the quantities $\epsilon_{u}$ and $N_{\mathrm{bkg}}$ would lead to a complete dependence on the simulation of the background and of the decay of the other $B$ meson in the event, resulting in large systematic uncertainties. These are substantially reduced by the use of two control samples. First, the $D^{0} e \bar{\nu}_{e} X$ control sample is used to reduce the $\mathcal{B}_{u}^{\text {SL }}$ extraction sensitivity 


\begin{tabular}{|l|l|}
\hline Variable & Definition \\
\hline \hline$\epsilon_{u}$ & Selection efficiency for $B \rightarrow X_{u} e \bar{\nu}_{e}$ events (data) \\
$\epsilon_{u}^{\mathrm{MC}}$ & Selection efficiency for $B \rightarrow X_{u} e \bar{\nu}_{e}$ events (MC) \\
$f_{u}$ & Selected fraction of $B \rightarrow X_{u} e \bar{\nu}_{e}$ phase-space (target region in MC) \\
$\epsilon_{\mathrm{sig}}$ & Selection efficiency for target $B \rightarrow X_{u} e \bar{\nu}_{e}$ events (MC) \\
$\epsilon_{\overline{\mathrm{sig}}}^{\mathrm{data}}$ & Selection efficiency for non-target $B \rightarrow X_{u} e \bar{\nu}_{e}$ events (MC) \\
$\epsilon_{D^{0} e \nu}^{\mathrm{MC}}$ & Selection efficiency for $B \rightarrow D^{0} e \bar{\nu}_{e} X$ events* (data) \\
$\epsilon_{D^{0} e \nu}^{\mathrm{eu}}$ & Selection efficiency for $B \rightarrow D^{0} e \bar{\nu}_{e} X$ events* $(\mathrm{MC})$ \\
$N_{\text {cand }}^{\text {ata }}$ & Total yield for signal region $($ data) \\
$M_{\mathrm{bkg}}^{\mathrm{MC}}$ & Background yield for signal region $(\mathrm{MC})$ \\
$N_{\mathrm{sita}}^{\mathrm{data}}$ & Yield for $s_{\mathrm{h}}^{\max }>4.25 \mathrm{GeV}^{2}$ sideband region (data) \\
$M_{\mathrm{side}}^{\mathrm{MC}}$ & Yield for $s_{\mathrm{h}}^{\max }>4.25 \mathrm{GeV}^{2}$ sideband region $(\mathrm{MC})$ \\
\hline
\end{tabular}

Table 7.1: Summary of the variables used to extract $\mathcal{B}_{u}^{\mathrm{SL}}$ and $\Delta \mathcal{B}_{u}^{\mathrm{SL}}$.

to the signal efficiency prediction, as discussed in section 6.5. Furthermore, the Monte Carlo background estimate is adjusted by comparing the yields in data and Monte Carlo for the sideband region $s_{\mathrm{h}}^{\max }>4.25 \mathrm{GeV}^{2}$ displayed in figure 5.14, as most of the events entering this sideband are background events. In fact, $B \rightarrow X_{u} e \bar{\nu}_{e}$ decays contribute to only $\sim 2 \%$ of the total sideband yield computed from the simulation. Hence, the efficiency and background estimates are adjusted as

$$
\epsilon_{u}=\epsilon_{u}^{\mathrm{MC}} \frac{\epsilon_{D^{0} e \nu}^{\mathrm{data}}}{\epsilon_{D^{0} e \nu}^{\mathrm{MC}}} \text { and } N_{\mathrm{bkg}}=M_{\mathrm{bkg}}^{\mathrm{MC}} \frac{N_{\text {side }}^{\mathrm{data}}}{M_{\text {side }}^{\mathrm{MC}}},
$$

such that equation 7.1 may be rewritten as

$$
\mathcal{B}_{u}^{\mathrm{SL}}=\frac{N_{\text {cand }}^{\text {data }}-M_{\mathrm{bkg}}^{\mathrm{MC}} \frac{N_{\text {side }}^{\text {data }}}{M_{\text {side }}^{\mathrm{MC}}}}{2 N_{B \bar{B}} \epsilon_{u}^{\mathrm{MC}} \frac{\epsilon_{D^{0}{ }_{e \nu}}^{\text {data }}}{\epsilon_{D^{0} e \nu}^{\mathrm{MC}}}} .
$$

Table 7.1 summarizes the variables used in the above equations and provides a brief description of their origin.

As the theoretical community is very active and new results are being published on a monthly basis, it is important to provide an experimental measurement with little sensitivity to theoretical models such that this result may be used in the context of an 
improved theory. The determination of a partial branching ratio, $\Delta \mathcal{B}_{u}^{\mathrm{SL}}$, unfolded for the detector efficiency and the boost of the $B$ meson fulfill this need. For this purpose, the signal efficiency may be expanded as

$$
\epsilon_{u}=\epsilon_{\mathrm{sig}} f_{u}+\epsilon_{\operatorname{sig}}\left(1-f_{u}\right),
$$

where $\epsilon_{\mathrm{sig}}$ is the efficiency for reconstructing a target event in the selected signal region, $\epsilon \overline{\mathrm{sig}}$ is the efficiency for reconstructing a non-target event in the selected signal region, and $f_{u}$ is the fraction of $B \rightarrow X_{u} \ell \bar{\nu}_{\ell}$ decays that fall in the true signal region as obtained in theoretical calculations. The target is the region defined in terms of the unsmeared kinematic variables $s_{\mathrm{h}}^{\max }$ and $E_{e}$ in the $B$-meson rest frame. The unfolded partial branching ratio is then extracted as

$$
\begin{aligned}
\Delta \mathcal{B}_{u}^{\mathrm{SL}} & =\mathcal{B}_{u}^{\mathrm{SL}} f_{u} \\
& =\frac{N_{\text {cand }}^{\text {data }}-N_{\mathrm{bkg}}}{\epsilon_{u} N_{B}} f_{u} \\
& =\frac{N_{\text {cata }}^{\text {data }}-N_{\mathrm{bkg}}}{\left(\epsilon_{\mathrm{sig}} f_{u}+\epsilon_{\mathrm{sig}}\left(1-f_{u}\right)\right) N_{B}} f_{u} \\
& =\frac{N_{\text {cand }}^{\text {data }}-M_{\mathrm{bkg}}^{\mathrm{MC}}}{\epsilon_{\mathrm{sig}} N_{B}}\left[1+\left(\frac{1}{f_{u}}-1\right) \frac{\epsilon_{\overline{\mathrm{sig}}}}{\epsilon_{\mathrm{sig}}}\right]^{-1} .
\end{aligned}
$$

Since the ratio $\epsilon_{\overline{\mathrm{sig}}} / \epsilon_{\mathrm{sig}}$ is small, $\Delta \mathcal{B}_{u}^{\mathrm{SL}}$ depends only weakly on the model used, i.e. it depends only weakly on $f_{u}$. The unfolded partial branching ratio described in equation 7.5 is adjusted similarly using the quantity listed in equation 7.2 such that

$$
\Delta \mathcal{B}_{u}^{\mathrm{SL}}=\frac{N_{\text {cand }}^{\text {data }}-M_{\mathrm{bkg}}^{\mathrm{MC}} \frac{N_{\mathrm{side}}^{\text {data }}}{M_{\mathrm{side}}^{\mathrm{de}}}}{2 N_{B \bar{B}} \epsilon_{\operatorname{sig}} \frac{\epsilon_{D^{0} 0_{e \nu}}^{\mathrm{dat}}}{\epsilon_{D^{0}{ }_{e \nu}}^{\mathrm{NC}}}}\left[1+\left(\frac{1}{f_{u}}-1\right) \frac{\epsilon_{\overline{\mathrm{sig}}}}{\epsilon_{\mathrm{sig}}}\right]^{-1} .
$$

Figure 7.1 displays the $q^{2}-E_{e}^{*}$ signal region for reconstructed events satisfying the event selection; also shown are the generated quantities for these events. Hence, a small fraction of events generated outside the signal (target) region feeds into the signal region due to the resolution on $E_{e}^{*}$ and, more likely, due to a mis-reconstruction of $q^{2}$. The target region is $\tilde{E}_{e}>2.0 \mathrm{GeV}$ and $s_{\mathrm{h}}^{\max }<3.5 \mathrm{GeV}^{2}$, a choice that roughly minimizes $\epsilon \overline{\mathrm{sig}} / \epsilon_{\text {sig }}$ and thus the dependence of the unfolding on the theoretical model used to determine $f_{u}$. 


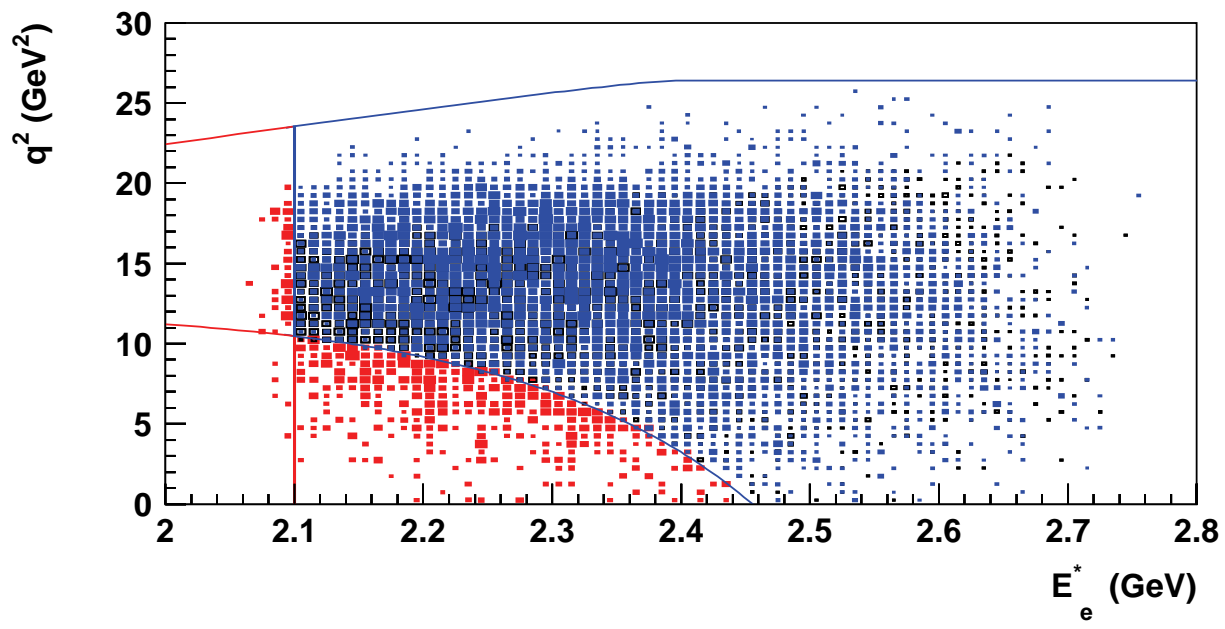

Figure 7.1: Generated $B \rightarrow X_{u} \ell \bar{\nu}_{\ell}$ events which have been reconstructed in the $q^{2}-E_{e}^{*}$ signal region. As can be seen, events generated outside the signal (target) region may feed into the signal region due to poor $E_{e}^{*}$ or, more likely, $q^{2}$ reconstruction.

Prior to performing a measurement of the (partial) $B \rightarrow X_{u} \ell \bar{\nu}_{\ell}$ branching ratio, systematic uncertainties must be evaluated to validate the above extraction procedure. The following sections discuss the various source of systematics and their effect on the signal extraction. The evaluation of the branching ratio is performed in chapter 8 once these effects are well understood.

\subsection{Procedure for evaluating uncertainties}

The nominal Monte Carlo yields and efficiencies used for the measurement of the (partial) $\mathcal{B}\left(B \rightarrow X_{u} \ell \bar{\nu}_{\ell}\right)$ branching fraction are listed in table 7.2. Various uncertainties may affect one or many of the quantities used to compute $\mathcal{B}_{u}^{\mathrm{SL}}$ and $\Delta \mathcal{B}_{u}^{\mathrm{SL}}$ such as the choice of HQE parameters used in signal modeling, uncertainties in the charm background branching ratios and modeling of the detector. Therefore, the simulation is adjusted to reflect the effect of each systematic uncertainty. For instance, in order to study the systematics due 


\begin{tabular}{|l|l|l|l|l|}
\hline$N_{\text {cand }}^{\text {nomi }}$ & $f_{u} \times 10^{-3}$ & $\epsilon_{\text {sig }} \times 10^{-3}$ & $\epsilon_{\overline{\text { sig }}} \times 10^{-3}$ & $\epsilon_{u}^{\mathrm{MC}} \times 10^{-3}$ \\
$5473 \pm 42$ & $140.1 \pm 0.5$ & $30.1 \pm 0.3$ & $0.56 \pm 0.020$ & $4.68 \pm 0.05$ \\
\hline$\epsilon_{\text {Dev }}^{\text {nomi }} \times 10^{-3}$ & $M_{\text {cand }}^{\text {bkg }}$ & $N_{\text {side }}^{\text {nomi }}$ & $\mathcal{B}_{u}^{\mathrm{SL}} \times 10^{-3}$ & $\Delta \mathcal{B}_{u}^{\mathrm{SL}} \times 10^{-4}$ \\
$90.6 \pm 1.9$ & $3176 \pm 35$ & $6423 \pm 49$ & $2.78 \pm 0.20$ & $3.88 \pm 0.28$ \\
\hline
\end{tabular}

Table 7.2: Nominal Monte Carlo yields and efficiencies. The uncertainties are purely statistical and the branching fraction uncertainty includes uncertainties from off-peak data.

to uncertainties in the simulation of the tracking efficiency, Monte Carlo simulations are reprocessed, randomly discarding tracks to simulate a reduced efficiency. This changes $M_{\mathrm{bkg}}^{\mathrm{MC}}$, $M_{\text {side }}^{\mathrm{MC}}, \epsilon_{u}^{\mathrm{MC}}$ and $\epsilon_{D e \nu}^{\mathrm{MC}}$. The signal extraction procedure is then reapplied and a measurement of an alternative $\mathcal{B}_{u}^{\mathrm{SL}}$,

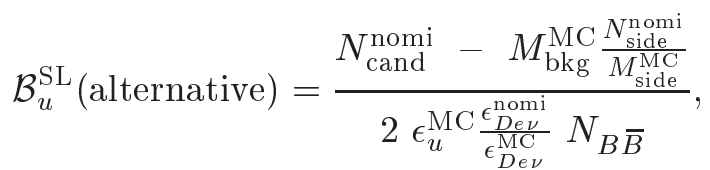

is performed, where $N_{\text {cand }}^{\text {nomi }}, N_{\text {side }}^{\text {nomi }}$, and $\epsilon_{D e \nu}^{\text {nomi }}$ correspond to the yields and efficiencies computed with the nominal Monte Carlo simulation as appearing in table 7.2. The difference observed in the extracted branching ratio with respect to the nominal value,

$$
\pm \mid \mathcal{B}_{u}^{\mathrm{SL}} \text { (alternative) }-\mathcal{B}_{u}^{\mathrm{SL}} \mid
$$

is taken as the systematic uncertainty. This procedure is repeated for each source of systematic error. The total uncertainty is then computed as the quadratic sum of all of the individual uncertainties.

\subsection{Modeling of $B \rightarrow X_{u} e \bar{\nu}_{e}$ signal events}

The systematic uncertainties inherent to the hybrid model are first evaluated by considering the uncertainty from the HQE parameters, $m_{b}$ and $a$, which affect the non-resonant component of the hybrid model as discussed in sections 2.5.6 and 4.2.2. As mentioned in section 2.5.6, three ansätze are available in the literature to parameterize the shape function. The nominal ansatz in this analysis follows the exponential form. A systematic uncertainty 
is therefore assigned to the branching ratio measurement from the difference observed in the extracted value using the alternative shape functions. These ansätze, including the exponential form, must satisfy the conditions imposed on the first three $k_{+}$moments, namely: $A_{0}=1, A_{1}=0$, and $A_{2}=-\lambda_{1} / 3$. Using the $B \rightarrow X_{s} \gamma$ result from [40], these criteria are used to solve for the parameters $m_{b}$ and $a$ of the various shape functions [41]. The list of extracted parameters and corresponding $\mathcal{B}_{u}^{\mathrm{SL}}$ and $\Delta \mathcal{B}_{u}^{\mathrm{SL}}$ extracted values are presented in table 7.3. The maximum deviations $\left( \pm\right.$ ) observed on the extracted $\mathcal{B}_{u}^{\mathrm{SL}}$ and $\Delta \mathcal{B}_{u}^{\mathrm{SL}}$ using the various HQE parameter ellipses are defined as the SF systematic uncertainties. For instance, the largest deviations observed on the extraction of $\mathcal{B}_{u}^{\mathrm{SL}}$ are observed using the Exponential SF with parameters $\left\{m_{b}, a\right\}=\left\{4.57 \mathrm{GeV} / c^{2}, 2.44\right\}$ and Gaussian SF with parameters $\left\{m_{b}, a\right\}=\left\{4.701 \mathrm{GeV} / c^{2}, 1.04\right\}$. Accordingly, the systematic uncertainty on $\mathcal{B}_{u}^{\mathrm{SL}}$ is evaluated to be $10.1 \%$. As expected, the SF uncertainty on $\Delta \mathcal{B}_{u}^{\mathrm{SL}}$ is much lower, about $\pm 1.3 \%$ even though $f_{u}$ varies by $(+11.2 /-8.9) \%$. This is a feature of the unfolding procedure: $\epsilon_{\mathrm{sig}} / \epsilon_{\mathrm{sig}}$ is small (about 0.019), such that the dependence on $f_{u}$ is suppressed (see equation 7.6).

Further systematic uncertainties arise from to the modeling of the resonant $B \rightarrow$ $X_{u} e \bar{\nu}_{e}$ sample. To evaluate the corresponding error on $\mathcal{B}_{u}^{\mathrm{SL}}$, the branching fractions for the resonant component (see table 4.2) of the hybrid model are scaled by their uncertainties coherently for charged and neutral $B$ decays. New hybrid models corresponding to the revised exclusive branching fractions are computed using the usual prescription, and are used to evaluate the systematic uncertainty on the $B \rightarrow X_{u} e \bar{\nu}_{e}$ branching fraction. Uncertainties due to the hybridization of the signal simulation are then evaluated by comparing the fully non-resonant signal Monte Carlo with the nominal hybrid. The $B \rightarrow X_{u} e \bar{\nu}_{e}$ simulation results in uncertainties of $4.1 \%$ on $\mathcal{B}_{u}^{\mathrm{SL}}$, and $4.4 \%$ on $\Delta \mathcal{B}_{u}^{\mathrm{SL}}$.

The modeling of the hadronization process from the breaking of the $X_{u}$ particle in JETSET was also investigated. To do so, the branching ratio obtained using the purely non-resonant signal sample was compared with the result obtained using the hybrid and 


\begin{tabular}{|c|c|c|c|c|c|c|}
\hline MC signal type & $f_{u}$ & $\epsilon_{\mathrm{sig}}$ & $\epsilon_{\overline{\mathrm{sig}}}$ & $\epsilon_{u}^{\mathrm{MC}}$ & $\mathcal{B} \times 10^{-3}$ & $\Delta \mathcal{B} \times 10^{-4}$ \\
\hline $\begin{array}{l}\text { Nominal } \\
\{m b, a\}=\{4.62,2.27\}\end{array}$ & 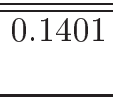 & 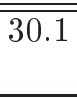 & 0.56 & 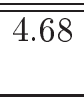 & 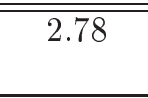 & 3.88 \\
\hline Non-resonant only & 0.1394 & 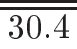 & 0.51 & 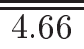 & $\overline{2.76}$ & 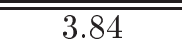 \\
\hline $\mathcal{B}\left(B \rightarrow \pi e \bar{\nu}_{e}\right)(-30 \%)$ & 0.1401 & 30.7 & 0.57 & 4.76 & 2.73 & 3.81 \\
\hline $\mathcal{B}\left(B \rightarrow \pi e \bar{\nu}_{e}\right)(+30 \%)$ & 0.1402 & 29.5 & 0.55 & 4.59 & 2.83 & 3.95 \\
\hline $\mathcal{B}\left(B \rightarrow \rho e \bar{\nu}_{e}\right)(-30 \%)$ & 0.1404 & 29.4 & 0.56 & 4.59 & 2.83 & 3.96 \\
\hline $\mathcal{B}\left(B \rightarrow \rho e \bar{\nu}_{e}\right)(+30 \%)$ & 0.1399 & 30.7 & 0.56 & 4.76 & 2.73 & 3.80 \\
\hline $\mathcal{B}\left(B \rightarrow \omega e \bar{\nu}_{e}\right)(-40 \%)$ & 0.1402 & 29.6 & 0.56 & 4.62 & 2.81 & 3.93 \\
\hline $\mathcal{B}\left(B \rightarrow \omega e \bar{\nu}_{e}\right)(+40 \%)$ & 0.1400 & 30.5 & 0.56 & 4.73 & 2.75 & 3.83 \\
\hline $\mathcal{B}\left(B \rightarrow \eta^{\left({ }^{\prime}\right)} e \bar{\nu}_{e}\right)(-100 \%)$ & 0.1404 & 29.0 & 0.54 & 4.53 & 2.86 & 4.00 \\
\hline $\mathcal{B}\left(B \rightarrow \eta^{\left({ }^{\prime}\right)} e \bar{\nu}_{e}\right)(+100 \%)$ & 0.1398 & 31.1 & 0.57 & 4.82 & 2.70 & 3.76 \\
\hline$\overline{\mid c m b, a\}=\{4.68,2.87\}}$ & $\overline{0.1518}$ & 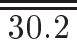 & 0.60 & 5.08 & 2.57 & 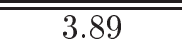 \\
\hline$\{m b, a\}=\{4.64,3.89\}$ & 0.1399 & 29.6 & 0.59 & 4.63 & 2.82 & 3.92 \\
\hline$\{m b, a\}=\{4.64,1.45\}$ & 0.1486 & 30.6 & 0.56 & 5.01 & 2.60 & 3.84 \\
\hline$\{m b, a\}=\{4.60,1.23\}$ & 0.1328 & 29.5 & 0.56 & 4.39 & 2.96 & 3.91 \\
\hline$\{m b, a\}=\{4.60,3.20\}$ & 0.1428 & 30.6 & 0.54 & 4.81 & 2.70 & 3.83 \\
\hline$\{m b, a\}=\{4.57,2.44\}$ & 0.1281 & 29.7 & 0.53 & 4.24 & 3.05 & 3.89 \\
\hline$\{m b, a\}=\{4.57,2.45\}$ & 0.1365 & 30.5 & 0.52 & 4.60 & 2.82 & 3.83 \\
\hline$\{m b, a\}=\{4.53,1.53\}$ & 0.1276 & 30.1 & 0.50 & 4.26 & 3.03 & 3.85 \\
\hline$\{m b, a\}=\{4.65,0.64\}$ & 0.1443 & 30.1 & 0.57 & 4.82 & 2.71 & 3.90 \\
\hline$\{m b, a\}=\{4.701,1.04\}$ & 0.1561 & 30.4 & 0.61 & 5.24 & 2.50 & 3.89 \\
\hline$\{m b, a\}=\{4.66,1.72\}$ & 0.1438 & 29.7 & 0.60 & 4.77 & 2.75 & 3.93 \\
\hline$\{m b, a\}=\{4.66,0.46\}$ & 0.1495 & 30.6 & 0.56 & 5.04 & 2.59 & 3.86 \\
\hline$\{m b, a\}=\{4.64,1.61\}$ & 0.1393 & 29.6 & 0.58 & 4.61 & 2.84 & 3.93 \\
\hline$\{m b, a\}=\{4.64,0.43\}$ & 0.1457 & 30.6 & 0.55 & 4.90 & 2.66 & 3.86 \\
\hline$\{m b, a\}=\{4.62,1.41\}$ & 0.1352 & 29.6 & 0.57 & 4.47 & 2.92 & 3.93 \\
\hline$\{m b, a\}=\{4.62,0.44\}$ & 0.1417 & 30.5 & 0.54 & 4.76 & 2.73 & 3.86 \\
\hline$\{m b, a\}=\{4.584,0.74\}$ & 0.1319 & 30.0 & 0.53 & 4.39 & 2.96 & 3.88 \\
\hline$\{m b, a\}=\{4.62,0.78\}$ & 0.1400 & 30.1 & 0.55 & 4.67 & 2.79 & 3.89 \\
\hline$\{m b, a\}=\{4.682,1.01\}$ & 0.1524 & 30.3 & 0.60 & 5.10 & 2.57 & 3.90 \\
\hline$\{m b, a\}=\{4.655,1.46\}$ & 0.1441 & 29.8 & 0.59 & 4.78 & 2.74 & 3.93 \\
\hline$\{m b, a\}=\{4.655,0.47\}$ & 0.1504 & 30.6 & 0.56 & 5.07 & 2.58 & 3.86 \\
\hline$\{m b, a\}=\{4.61,1.27\}$ & 0.1345 & 29.6 & 0.56 & 4.45 & 2.93 & 3.93 \\
\hline$\{m b, a\}=\{4.61,0.35\}$ & 0.1428 & 30.6 & 0.54 & 4.81 & 2.71 & 3.85 \\
\hline$\{m b, a\}=\{4.58,1.00\}$ & 0.1298 & 29.7 & 0.54 & 4.30 & 3.03 & 3.91 \\
\hline$\{m b, a\}=\{4.58,0.35\}$ & 0.1372 & 30.5 & 0.52 & 4.61 & 2.82 & 3.85 \\
\hline$\{m b, a\}=\{4.548,1.80\}$ & 0.1283 & 30.0 & 0.51 & 4.28 & 3.03 & 3.88 \\
\hline
\end{tabular}

Table 7.3: Systematic effects due to uncertainties within the signal simulation. Negligible variations are observed in the evaluation of $M_{\mathrm{bkg}}^{\mathrm{MC}}$ and $M_{\mathrm{side}}^{\mathrm{MC}}$. Hence, these values are not displayed in the above table. The last three blocks of values refer to the shape function uncertainties using Exponential, Gaussian, or Roman ansatz, respectively. Note that all of the above efficiencies are expressed in units of $\times 10^{-3}$. 
a)

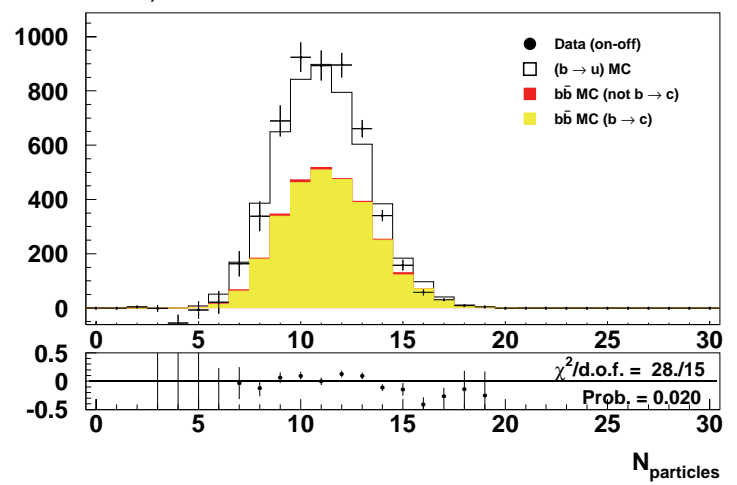

b)

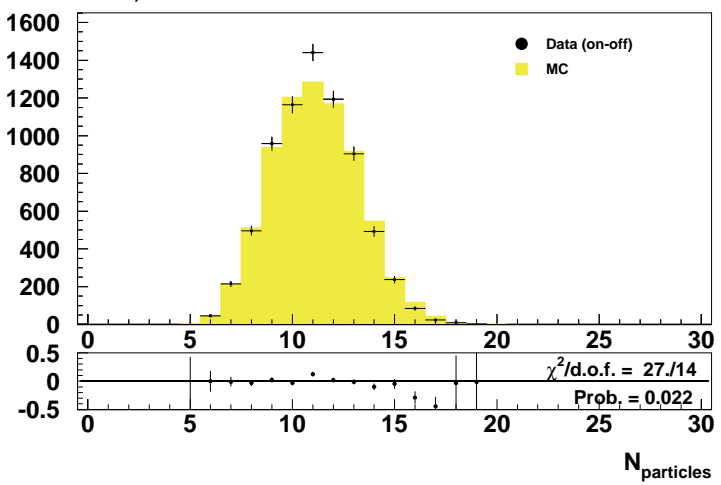

Figure 7.2: Number of particles per event for the a) signal and b) $D^{0} e \bar{\nu}_{e}$ control sample. After removing the $D^{(*)}$ daughters, the multiplicity from the other $B$-meson decay is found to be on average $\sim 7$.

only a small difference was found, as shown in table 7.3. As the signal efficiency decreases with more particles produced in a given event, the multiplicity of the signal events was also taken into account. The event multiplicity was first evaluated in both data and MC using the control sample as shown in figure 7.2. Under the assumption that the multiplicity of the $B \rightarrow D^{0} e \bar{\nu}_{e} X$ was properly modeled, the multipliciy of the other $B$ meson decay appears to be reasonable, on average about $\sim 7$ for both data and simulation. A good agreement was also found for the total event multiplicity based on the signal sample. Hence the multiplicity from $B \rightarrow X_{u} e \bar{\nu}_{e}$ decays appears to be properly modeled, and systematic uncertainties due to the hadronization process are assumed to be negligible.

\subsection{Background simulation}

In simulating background events, several decay channels must be considered. Tables 6.1 and 7.4 summarizes the principal beauty to charm and charm decay modes, respectively, along with the corresponding branching fractions. The uncertainties listed in these tables correspond to current world average measurements [18], or to measurements performed at 


\begin{tabular}{|l|l|}
\hline Decay type & Branching ratio \\
\hline \hline$D^{0} \rightarrow K^{0} X$ & $0.42 \pm 0.05$ \\
$D^{+} \rightarrow K^{0} X$ & $0.59 \pm 0.07$ \\
$D_{s} \rightarrow K^{0} X$ & $0.39 \pm 0.28$ \\
\hline$D^{0} \rightarrow e X$ & $0.0687 \pm 0.0028$ \\
$D^{+} \rightarrow e X$ & $0.172 \pm 0.019$ \\
$D_{s} \rightarrow e X$ & $0.08 \pm 0.06$ \\
\hline
\end{tabular}

Table 7.4: Branching ratio for inclusive $D$ decay modes [18].

$B A B A R$, and affect background estimates. In order to evaluate the resulting systematic effects on the signal branching ratio, the procedure outlined in section 7.2 is followed. In computing the alternative $\mathcal{B}_{u}^{\mathrm{SL}}$, the Monte Carlo is adjusted such that the branching fraction of the decay type studied, e.g. the inclusive $D^{0} \rightarrow K^{0}$, matches the value \pm the uncertainty on this measurement. Figure 7.3 display various kinematical distributions after modifying the inclusive $D^{0} \rightarrow K^{0}$ branching ratio accordingly. Although only small changes are observed in these distributions, a $3 \%$ variation is obtained on the extracted (partial) branching ratio. In fact, the extraction procedure has no handle on the modeling of charm meson decays and results in an overall uncertainty of about $4.5 \%$ for both $\mathcal{B}_{u}^{\mathrm{SL}}$ and $\Delta \mathcal{B}_{u}^{\mathrm{SL}}$.

In reweighting the exclusive $B \rightarrow D^{(*, * *)} e \bar{\nu}_{e}$ channels, the overall semileptonic $B \rightarrow$ $X_{c} e \bar{\nu}_{e}$ branching ratio is kept constant by modifying the non-resonant $B \rightarrow \operatorname{Dn}(\pi) e \bar{\nu}_{e}$ branching ratio accordingly. If this constraint necessitates setting the branching fraction of the non-resonant decays to a negative value, the contribution from $B \rightarrow \operatorname{Dn}(\pi) e \bar{\nu}_{e}$ is set to zero and the branching fraction for the broad $B \rightarrow D^{* *} e \bar{\nu}_{e}$ is adjusted. To evaluate an uncertainty from the $B \rightarrow D^{* *} e \bar{\nu}_{e}$ simulation, the composition of the sample is modified such that all of the $D^{* *}$ states are either: narrow $D_{1}$ and $D_{2}^{*}(\mathrm{~N})$, broad $D_{0}^{*}$ and $D_{1}^{\prime}(\mathrm{B})$, or non-resonant Goity-Robert (GR) types. The changes on the extracted $\mathcal{B}_{u}^{\mathrm{SL}}$ and $\Delta \mathcal{B}_{u}^{\mathrm{SL}}$ due to the variations in the background branching fractions are presented in table 7.5.

The impact of varying the form factors for $B \rightarrow D^{(*)} e \bar{\nu}_{e}$ decays has been evaluated as well and the corresponding systematic uncertainties are listed in table 7.5. In the case 

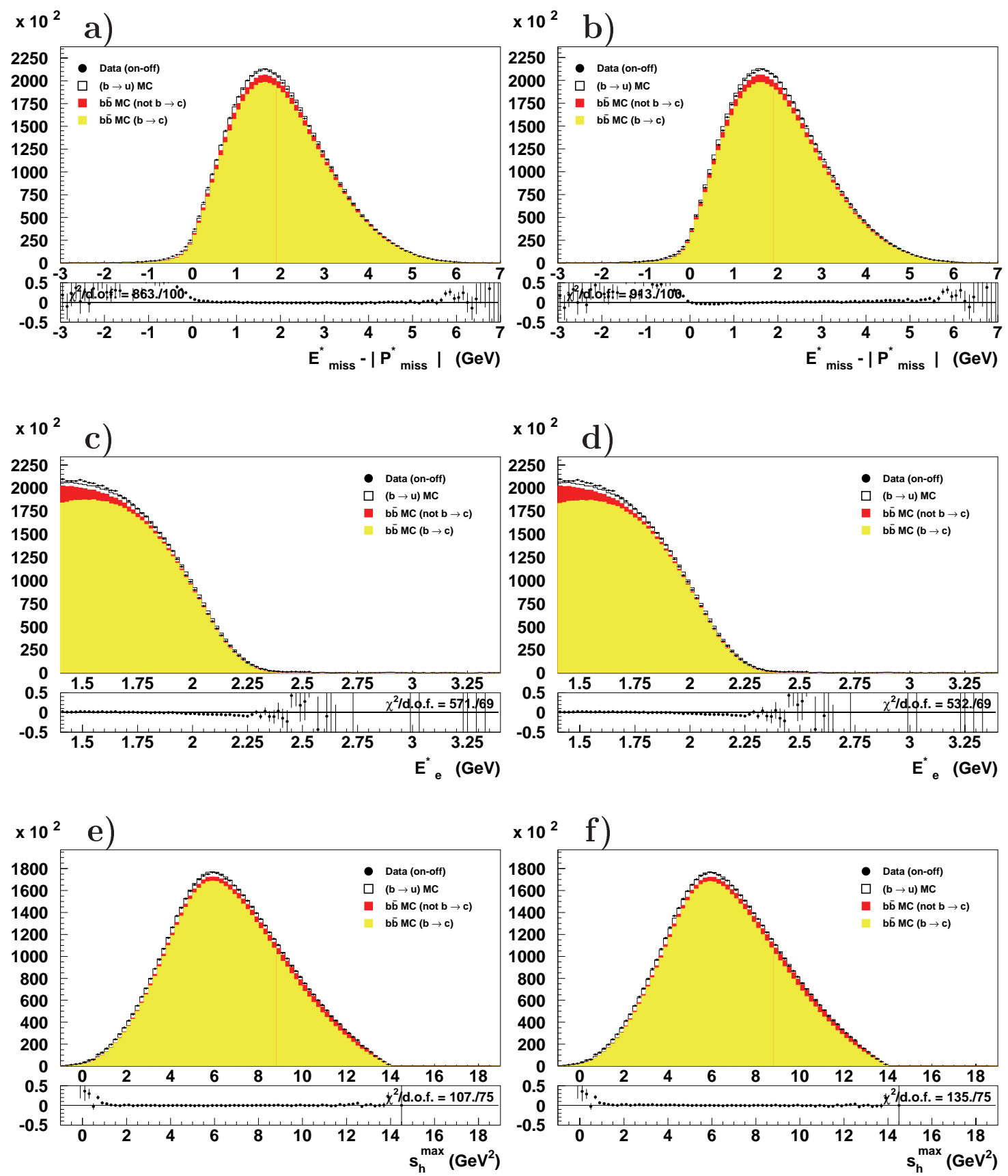

Figure 7.3: Uncertainty on $\mathcal{B}\left(D^{0} \rightarrow K^{0}\right.$ incl.) and its impact on the reconstruction of a) $E_{\text {miss }}^{*}-\left|\vec{P}_{\text {miss }}^{*}\right|$, c) $E_{e}^{*}$, and e) $s_{\mathrm{h}}^{\max }$. for branching ratio smaller (left) or greater (right) than the nominal one. These plots follow the same conventions as those of figure 5.14. 


\begin{tabular}{|l|l|c|c|c|c|c|c|}
\hline Decay & $\Delta$ & $\epsilon_{u}^{\mathrm{MC}} \times 10^{-3}$ & $\epsilon_{\text {Dev }}^{\mathrm{MC}} \times 10^{-3}$ & $M_{\text {cand }}^{\text {bkg }}$ & $M_{\text {side }}^{\mathrm{MC}}$ & $\mathcal{B} \times 10^{-3}$ & $\Delta \mathcal{B} \times 10^{-4}$ \\
\hline \hline Nominal MC & & 4.675 & 90.5 & 3176 & 6423 & 2.78 & 3.88 \\
\hline \hline$D^{0} \rightarrow K^{0}$ & $\downarrow$ & 4.766 & 91.5 & 3207 & 6673 & 2.86 & 4.00 \\
incl. & $\uparrow$ & 4.585 & 89.5 & 3143 & 6171 & 2.69 & 3.75 \\
\hline$D^{+} \rightarrow K^{0}$ & $\downarrow$ & 4.708 & 91.2 & 3183 & 6492 & 2.82 & 3.93 \\
incl. & $\uparrow$ & 4.642 & 89.8 & 3168 & 6350 & 2.74 & 3.83 \\
\hline$D_{s}^{+} \rightarrow K^{0}$ & $\downarrow$ & 4.833 & 91.5 & 3290 & 6640 & 2.71 & 3.78 \\
incl. & $\uparrow$ & 4.518 & 89.5 & 3068 & 6198 & 2.84 & 3.96 \\
\hline$D^{0} \rightarrow e, \mu$ & $\downarrow$ & 4.678 & 90.7 & 3168 & 6434 & 2.80 & 3.91 \\
incl. & $\uparrow$ & 4.672 & 90.3 & 3184 & 6412 & 2.76 & 3.85 \\
\hline$D^{+} \rightarrow e, \mu$ & $\downarrow$ & 4.680 & 91.0 & 3107 & 6388 & 2.86 & 3.99 \\
incl. & $\uparrow$ & 4.668 & 90.2 & 3185 & 6405 & 2.75 & 3.84 \\
\hline$D_{s}^{+} \rightarrow e, \mu$ & $\downarrow$ & 4.678 & 90.6 & 3178 & 6426 & 2.78 & 3.88 \\
incl. & $\uparrow$ & 4.672 & 90.4 & 3175 & 6420 & 2.78 & 3.88 \\
\hline \hline$B \rightarrow D e \bar{\nu}_{e}$ & $\downarrow$ & 4.675 & 90.6 & 3156 & 6383 & 2.78 & 3.88 \\
& $\uparrow$ & 4.675 & 90.5 & 3197 & 6463 & 2.78 & 3.87 \\
\hline$B \rightarrow D^{*} e \bar{\nu}_{e}$ & $\downarrow$ & 4.675 & 90.5 & 3122 & 6324 & 2.79 & 3.89 \\
& $\uparrow$ & 4.675 & 90.5 & 3230 & 6522 & 2.77 & 3.87 \\
\hline$B \rightarrow D^{* *} e \bar{\nu}_{e}$ & $\mathrm{~N}$ & 4.675 & 90.7 & 3181 & 6458 & 2.80 & 3.91 \\
& $\mathrm{~B}$ & 4.675 & 90.3 & 3169 & 6396 & 2.77 & 3.86 \\
Composition & $\mathrm{GR}$ & 4.675 & 90.2 & 3174 & 6364 & 2.74 & 3.82 \\
\hline \hline$B \rightarrow X_{c} e \bar{\nu}_{e}$ & $\downarrow$ & 4.684 & 90.5 & 3125 & 6324 & 2.78 & 3.87 \\
& $\uparrow$ & 4.666 & 90.5 & 3228 & 6521 & 2.78 & 3.88 \\
\hline \hline$B \rightarrow D e \bar{\nu}_{e}$ & $\downarrow$ & 4.675 & 90.5 & 3173 & 6398 & 2.77 & 3.86 \\
Form factor & $\uparrow$ & 4.675 & 90.5 & 3180 & 6444 & 2.79 & 3.89 \\
\hline & $\mathrm{P} 1$ & 4.675 & 90.4 & 3197 & 6476 & 2.78 & 3.88 \\
$B \rightarrow D^{*} e \bar{\nu}_{e}$ & $\mathrm{P} 2$ & 4.675 & 90.6 & 3182 & 6429 & 2.78 & 3.88 \\
Form factors & $\mathrm{P} 3$ & 4.675 & 90.5 & 3168 & 6414 & 2.78 & 3.88 \\
& $\mathrm{P} 4$ & 4.675 & 90.4 & 3198 & 6472 & 2.78 & 3.88 \\
& $\mathrm{P} 5$ & 4.675 & 90.6 & 3154 & 6377 & 2.78 & 3.88 \\
& $\mathrm{P} 6$ & 4.675 & 90.6 & 3145 & 6356 & 2.78 & 3.88 \\
\hline
\end{tabular}

Table 7.5: Systematics due to charm and beauty to charm uncertainties in background modeling. $\Delta=\uparrow \downarrow$ indicates the upward and downward variation of the branching fraction and the $B \rightarrow D e \bar{\nu}_{e}$ form factor, respectively. P1 - P6 indicate the six points on the 1- $\sigma$ ellipsoid in the $\left(\rho^{2}, R_{1}, R_{2}\right)$ space as described in the text. The statistical uncertainties obtained on the above values are comparables to the ones listed in table 7.2. 
of $B \rightarrow D e \bar{\nu}_{e}$ decays, the Monte Carlo simulation relies on the ISGW2 model [23]. A pole form factor $F(w)=1-\rho^{2} \cdot(w-1)$, where $w=\left(m_{B}^{2}+m_{D}^{2}-q^{2}\right) /\left(2 m_{B} m_{D}\right)$, is used to describe the $B \rightarrow D e \bar{\nu}_{e}$ form factor based on the measured value of $\rho^{2}=0.76 \pm 0.16$ [65]. To evaluate the systematics due to the uncertainties on $\rho^{2}, B \rightarrow D e \bar{\nu}_{e}$ events are reweighted in the simulation by a factor $F\left(w, \rho^{2} \pm \sigma\right) / F\left(w, \rho^{2}\right)$ using a technique developed in Ref. [66]. In the case of $B \rightarrow D^{*} e \bar{\nu}_{e}$ decays, the uncertainty is evaluated similarly using recent form factor measurements [67]: $\rho^{2}=0.769 \pm 0.039 \pm 0.019 \pm 0.032, R_{1}=1.328 \pm 0.055 \pm 0.025 \pm 0.025$, and $R_{2}=0.920 \pm 0.044 \pm 0.020 \pm 0.013$. From the correlation matrix, six points $(\mathrm{P} 1$ P6) were defined along the 1- $\sigma$ ellipsoid in the $\left(\rho^{2}, R_{1}, R_{2}\right)$ phase-space in order to vary the HQET form factors in the simulation.

As a feature of the $B \rightarrow D^{(*, *)} e \bar{\nu}_{e}$ fit and $s_{\mathrm{h}}^{\max }$ sideband calculations, the measurement of the (partial) branching ratio has reduced sensitivity to the uncertainties in the $B \rightarrow D^{(*, * *)} e \bar{\nu}_{e}$ simulation, about $\pm 1.5 \%$, much less than the uncertainty due to the modeling of the subsequent $D$-meson decays.

\subsection{Tracking efficiency}

The tracking efficiencies for data and Monte Carlo simulations were evaluated in [68], where a track selection similar to the one used in this analysis was applied (see section 5.3.2). Charged tracks with transverse momentum $p_{t}>0.2 \mathrm{GeV}$ in the laboratory frame were studied using $\tau^{ \pm}$decays. Comparison between data and Monte Carlo showed that the simulation overestimated the efficiency by a factor $\epsilon_{\text {track }}^{\mathrm{MC}} / \epsilon_{\text {track }}^{\mathrm{data}}=0.5 \pm 0.5 \%$. As such, a systematic uncertainty is evaluated by removing an additional $0.5 \%$ of the tracks from the simulation. Efficiency study at low $p_{t}<0.2 \mathrm{GeV}$ were based on slow pion produced in $D^{*}$ decays and yield $\epsilon_{\text {track }}^{\mathrm{MC}} / \epsilon_{\mathrm{track}}^{\mathrm{data}}=1.000 \pm 0.016$. The systematic uncertainty for these low $p_{t}$ tracks is thus computed by removing randomly $-1.6 \%$ of the tracks in the simulation. Overall, these changes affect the extraction of the signal branching ratio by a factor $-1.8 \%$ when coherent changes are considered. The systematic uncertainty is symmetrized to $\pm 1.8 \%$ 


\begin{tabular}{|l|c|c|c|c|c|c|c|}
\hline Type & $\Delta$ & $\epsilon_{u}^{\mathrm{MC}} \times 10^{-3}$ & $\epsilon_{D e \nu}^{\mathrm{MC}} \times 10^{-3}$ & $M_{\text {cand }}^{\text {bkg }}$ & $M_{\text {side }}^{\mathrm{MC}}$ & $\mathcal{B} \times 10^{-3}$ & $\Delta \mathcal{B} \times 10^{-4}$ \\
\hline \hline Nominal MC & & 4.675 & 90.5 & 3176 & 6423 & 2.78 & 3.88 \\
\hline \hline Tracking eff. & & 4.587 & 89.3 & 3124 & 6230 & 2.74 & 3.83 \\
\hline$K_{S}$ eff. & & 4.675 & 90.8 & 3166 & 6408 & 2.79 & 3.90 \\
\hline Electron ID & $-\epsilon$ & 4.642 & 90.5 & 3153 & 6379 & 2.80 & 3.91 \\
& $+\epsilon$ & 4.708 & 90.5 & 3200 & 6470 & 2.76 & 3.85 \\
\hline$K^{ \pm}$ID & & 4.575 & 89.0 & 3043 & 6240 & 2.85 & 3.98 \\
\hline$\pi^{ \pm}$mis-ID & & 4.671 & 90.6 & 3165 & 6406 & 2.79 & 3.90 \\
\hline Neutral & 1 & 4.724 & 90.5 & 3238 & 6564 & 2.76 & 3.86 \\
& 2 & 4.502 & 87.3 & 3061 & 6043 & 2.69 & 3.76 \\
\hline$K_{L}^{0}$ energy & $-\sigma$ & 4.686 & 90.7 & 3178 & 6445 & 2.79 & 3.90 \\
& $+\sigma$ & 4.669 & 90.4 & 3166 & 6394 & 2.78 & 3.88 \\
\hline$K_{L}^{0}$ eff. & $-\epsilon$ & 4.715 & 91.2 & 3148 & 6517 & 2.87 & 4.00 \\
& $+\epsilon$ & 4.634 & 89.9 & 3178 & 6324 & 2.72 & 3.80 \\
\hline Bremss. & $1+$ & 4.698 & 90.9 & 3219 & 6470 & 2.76 & 3.86 \\
& $1-$ & 4.653 & 90.5 & 3123 & 6377 & 2.83 & 3.97 \\
(shift in $E_{e}^{*}$ ) & $2+$ & 4.693 & 91.0 & 3205 & 6468 & 2.78 & 3.89 \\
& $2-$ & 4.661 & 90.6 & 3135 & 6379 & 2.81 & 3.94 \\
\hline PHOTOS & $1+$ & 4.787 & 91.0 & 3233 & 6499 & 2.71 & 3.79 \\
& $1-$ & 4.587 & 90.3 & 3111 & 6363 & 2.87 & 4.02 \\
(vs Ginsberg) & $2+$ & 4.757 & 91.0 & 3215 & 6494 & 2.74 & 3.84 \\
& $2-$ & 4.609 & 90.5 & 3125 & 6360 & 2.85 & 3.99 \\
\hline
\end{tabular}

Table 7.6: Systematic effects due to uncertainties in the modeling of the detector performance such as tracking efficiency and EMC modeling.

since track killing cannot simulate the effect of improving the tracking efficiency. The results shown in table 7.6 demonstrate the benefit of the adjustments made on the signal extraction procedure given in section 7.1. In fact, using equation 7.1, the above changes in the tracking efficiency would lead to a $+3.6 \%$ variation on $\mathcal{B}_{u}^{\mathrm{SL}}$, whereas the adjusted extraction of equation 7.3 lead to a $-1.8 \%$ change only.

\subsection{Reconstruction of $K_{S}^{0}$}

The difference in the tracking reconstruction efficiencies between data and simulations has also an impact on the reconstruction efficiency of $K_{S}^{0}$ mesons in the decay $K_{S}^{0} \rightarrow \pi^{+} \pi^{-}$. According to [68], the ratio of data and Monte Carlo efficiencies for $K_{S}^{0}$ mesons reconstructed is unity, but a systematic uncertainty of $-2.6 \%$ needs to be assigned to the simulation. Hence, $K_{S}^{0}$ candidates are removed at random from the simulation with a probability of 
2.6\%. The daughter tracks are kept if they satisfy the charged track selection outlined in section 5.3.2. The effect on the extracted $\mathcal{B}_{u}^{\mathrm{SL}}$ is negligible, as shown in table 7.6. The change would be tiny even if the daughter tracks were always rejected, since the ratio of the $K_{S}^{0}$ to charged track multiplicity is about $1 / 40$ and the total number of tracks would be lowered by $2.6 \% \times 2 n_{K_{S}^{0}} / n_{\text {tracks }} \simeq 0.1 \%$. The variation taken in the overall tracking efficiency (section 7.5) is much larger than this.

\section{7 $\quad$ Electron identification}

The primary event selection requirement in this analysis is the presence of an identified electron with momentum above $1.4 \mathrm{GeV}$. Since the simulation is used to determine the efficiency of the selection, any difference in the performance of the electron identification between the simulation and data will cause a bias in the overall event selection. Therefore, the ratio of the electron identification efficiency in data to that in Monte Carlo was evaluated in bins of $p_{e}^{\text {lab }}\left(100 \mathrm{MeV}\right.$ wide) and $\theta^{\text {lab }}$ for electrons and positrons based on bhabha events. Using these tables, the simulated electron identification efficiency was adjusted to that of the data.

The uncertainty on the relative efficiencies was calculated from the statistical uncertainties on the electron/positron selection efficiency in data and in the simulation. The total uncertainty on $\mathcal{B}_{u}^{\mathrm{SL}}$ is then computed as the quadratic sum of the statistical error from each $\left\{p_{e}^{\mathrm{lab}}, \theta^{\mathrm{lab}}\right\}$ bin and the systematic error on to the correction factor. The latter was evaluated by comparing effiencies for simulated bhabha and $B \bar{B}$ events: $0.9925 \pm 0.0078$. Also note that the statiscal error for each $\left\{p_{e}^{\text {lab }}, \theta^{\text {lab }}\right\}$ bin was computed independently. This procedure results in the uncertainty on the branching fraction given in table 7.6.

The contribution of hadrons mis-identified as electrons is small, about $0.5 \%$ which comes from taking the product of the misidentification probabilities and the number of hadron tracks in the momentum region of interest and dividing by the number of identified signal electrons. Unfortunately, it is not well simulated, so while non-semileptonic events 
sometimes pass the event selection due to hadron mis-identification in the data, they rarely do so in the Monte Carlo and so are not in the analysis sample in sufficient numbers to be useful. Therefore, the full $0.5 \%$ is taken as a additional systematic uncertainty for the effect of hadron misidentification on $\mathcal{B}_{u}^{\mathrm{SL}}$ and $\Delta \mathcal{B}_{u}^{\mathrm{SL}}$ and is added in quadrature to the above uncertainty from electron identification uncertainty.

\subsection{Charged particle identification}

As discussed in section 5.3.5, the determination of the visible 4-momentum of the event requires a mass hypothesis to be assigned to each particle. Imperfect simulation of the performance of the particle identification can lead to systematic uncertainties in the visible 4-momentum reconstruction and affect the extracted signal. In order to test the sensitivity of $\mathcal{B}_{u}^{\mathrm{SL}}$ with respect to particle identification ${ }^{1}$, the mass of all charged tracks were set to the $\pi^{+}$mass. The resulting branching fraction obtained was within $3.1 \%$ of the nominal value. In a more realistic approach, the kaon identification efficiency and misidentification probabilities were varied, since kaons are the most common charged particles after pions (and signal electrons) in the selected events, about $8 \%$ of the total. According to [62], the kaon identification efficiency was varied by $-20 \%$, resulting in the changes listed in table 7.6.

Setting the rate for the mis-identification of pions as kaons to zero produced a tiny change in the branching fraction. Given that these uncertainties are already small, uncertainties due to the modeling of the efficiency and misidentification for protons and muons, which comprise only less than $2 \%$ of charged tracks in selected events, are neglected. The uncertainties described in this section are added in quadrature to obtain an overall $2.2 \%$ particle identification uncertainty on $\mathcal{B}_{u}^{\mathrm{SL}}$.

\footnotetext{
${ }^{1}$ This excludes the identification of the signal electron listed in section 7.7 .
} 


\subsection{Neutrals corrections and systematics}

There are differences in the detection of photons in the electromagnetic calorimeter between data and the simulations. Studies have been performed to adjust the simulation for these discrepencies [69] and corrections, which consist of an energy correction and a smearing of the photon energy, were implemented accordingly. The amount of smearing is energydependent in order to reproduce the energy resolution as measured in data. Hence, two additional uncertainties must be considered:

1. First, the photon efficiency is varied following a Gaussian probability density function with parameters depending on the photon energy. Photons are reweighted according to this PDF, and each event is assigned a weight equal to the product of the weights of all photons in the event. The difference observed in the extracted $\mathcal{B}_{u}^{\mathrm{SL}}$ with respect to the one obtained using the nominal neutral simulation provides the so-called uncorrelated uncertainty.

2. In the second case, $2.5 \%$ of the photons are randomly removed from the simulation. This killing is independent of the photon energy. This provides the so-called correlated uncertainty.

The effects of the correlated uncertainty are shown in figure 7.6 in terms of the neutral

multiplicity, total energy reconstructed in the EMC, and $E_{\text {miss }}^{*}-\left|\vec{P}_{\text {miss }}^{*}\right|$. A much better agreement in terms of the neutral multiplicity is achieved after applying this extra $2.5 \%$ killing, but the simulation of the total (neutral) energy reconstruction and $E_{\text {miss }}^{*}-\left|\vec{P}_{\text {miss }}^{*}\right|$ are clearly inadequate. The resulting uncertainties on the branching fraction are presented in table 7.6. These two errors are then added in quadrature to provide the total neutral systematic uncertainty. 

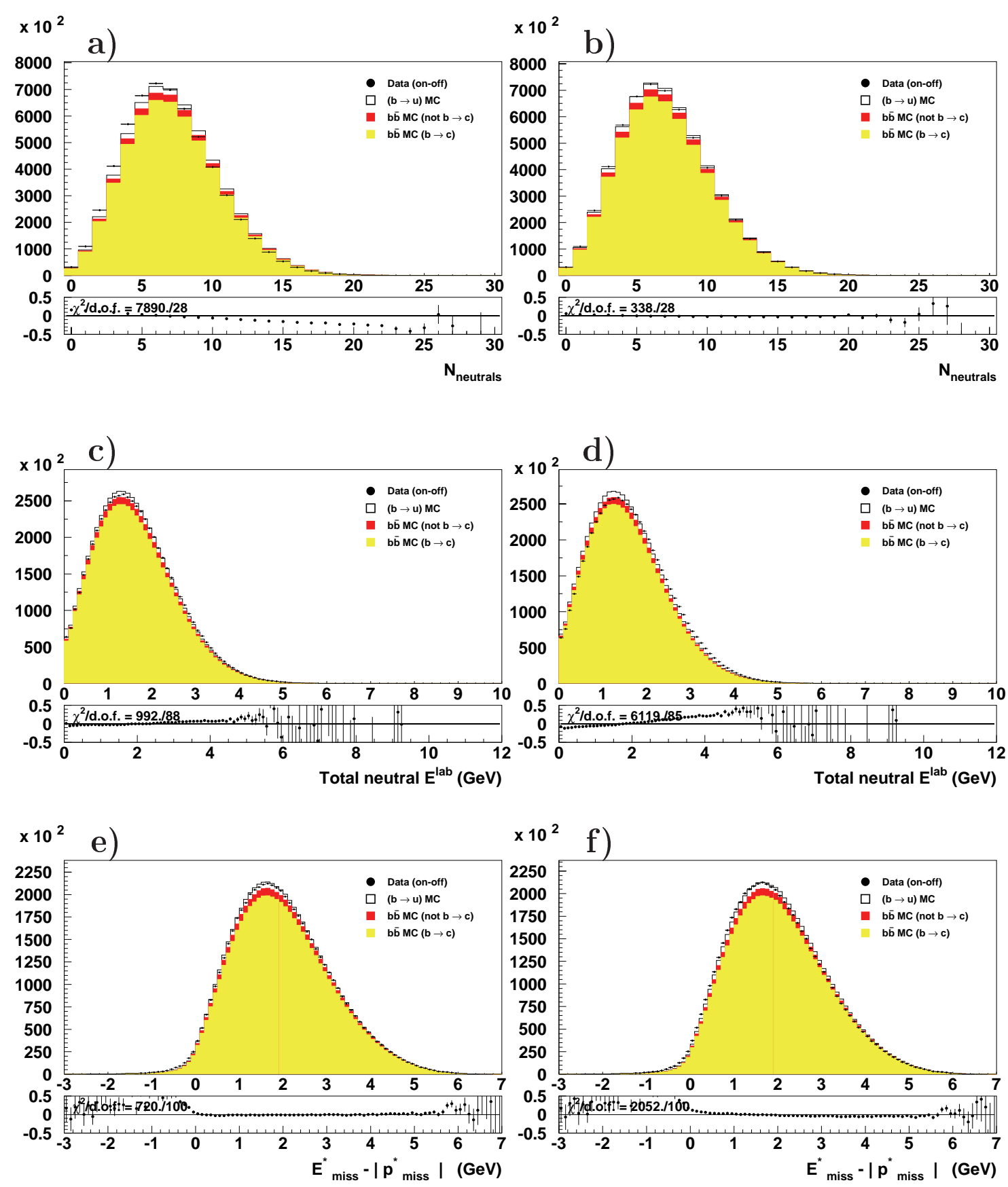

Figure 7.4: Effects of the correlated uncertainty in energy reconstruction in terms of a) neutral multiplicity, c) total neutral energy reconstruction and e) $E_{\text {miss }}^{*}-\left|\vec{P}_{\text {miss }}^{*}\right|$ prior to and after the $2.5 \%$ neutral killing ( $\mathrm{b}, \mathrm{d}$ and $\mathrm{f}$, respectively). These plots follow the same conventions as those of figure ??. 


\section{$7.10 \quad K_{L}^{0}$ reconstruction}

Although the neutrals selection criteria suppresses the selection of $K_{L}^{0}$ mesons, a nonnegligible amount of these mesons are used in the reconstruction of the visible energy. As a consequence, $q^{2}$ is sensitive to the modeling of the $K_{L}^{0}$ energy deposition in the electromagnetic calorimeter.

Many studies have shown that the momentum distribution for $K_{L}^{0}$ is adequately generated in Monte Carlo (e.g. [70]). In this analysis, a reasonable agreement was found between data and Monte Carlo when comparing the $K_{S}^{0}$ momentum distribution, which also suggests a proper simulation of $K_{L}^{0}$ momentum (section 6.4). Under the assumption that $K_{L}^{0}$ are well modeled in the event generator, the remaining question is how well the energy deposition in the EMC is simulated. In [70], $K_{L}^{0}$ mesons were selected using radiative return events, $e^{+} e^{-} \rightarrow \phi \gamma$ where $\phi \rightarrow K_{L}^{0} K_{S}^{0}$. Unfortunately, the $K_{L}^{0}$ selected this way are high-energetic mesons whereas the ones most influencing this analysis are produced in $D$ meson decays and have significantly less energy.

Instead, the energy deposition was evaluated using kinematically identified $K_{L}^{0}$ mesons produced in the decay chain $D^{*} \rightarrow D^{0} \pi_{\text {soft }}^{+}$, where the $D$ meson decayed to a $K_{L}^{0} \pi^{+} \pi^{-}$final state. Based on this selector, the ratio of the $K_{L}^{0}$ energy deposition between data and the simulation was parameterized as a function of the momentum of the particle as

$$
\frac{E_{\mathrm{cal}}^{\mathrm{data}}}{E_{\mathrm{cal}}^{\mathrm{MC}}}=a_{0}+a_{1} \cdot p_{K_{L}^{0}}+a_{2} \cdot p_{K_{L}^{0}}^{2}+a_{3} \cdot p_{K_{L}^{0}}^{3}
$$

for the momentum range $0.5 \mathrm{GeV}<p_{K_{L}^{0}}<2 \mathrm{GeV}$ [71]. Using the error matrix shown in table 7.7), a systematic uncertainty on this correction factor was determined from the $\pm 1 \sigma$ envelope and alternative $\mathcal{B}_{u}^{\mathrm{SL}}$ were computed accordingly, as shown in table 7.6.

Furthermore, studies based on the comparison between $D \rightarrow K_{L}^{0} \pi \pi$ and $D \rightarrow K_{S}^{0} \pi \pi$ have shown that the number of $K_{L}^{0}$ depositing energy in the EMC was overestimated, and an additional $-30 \pm 20 \%$ efficiency correction was applied to the simulation. The effect of the above uncertainties on $\mathcal{B}_{u}^{\mathrm{SL}}$ are shown in table 7.6. Hence, the overall error uncertainty 


\begin{tabular}{|c||c|c|c|c|}
\hline \hline Parameters & $a_{0}$ & $a_{1}$ & $a_{2}$ & $a_{3}$ \\
\hline \hline Fitted values & -0.8883 & 5.274 & -3.436 & 0.6476 \\
\hline \hline & 0.0829 & -0.128 & 0.046 & -0.00101 \\
Error & -0.1280 & 0.265 & -0.157 & 0.0266 \\
Matrix & 0.0460 & -0.157 & 0.148 & -0.0400 \\
& -0.00101 & 0.0266 & -0.040 & 0.0139 \\
\hline
\end{tabular}

Table 7.7: Parameterization results to adjust the ratio of $K_{L}^{0}$ energy deposition between data and Monte Carlo as a function of $p_{K_{L}^{0}}$. The fitted function is outlined in equation 7.8.

from the modeling of the $K_{L}^{0}$ energy deposition in the EMC is about $3 \%$ for both the partial and total branching ratio measurements.

\subsection{Bremsstrahlung emission}

Due to emission of bremsstrahlung photons, electrons lose energy when traversing the detector material. It has been shown that the bremsstrahlung modeling in the detector simulation agrees within statistical uncertainties with what has been observed on data using a Bhabha control sample [57]. According to this study, the difference in the amount of detector material between the interaction point and the EMC implemented in the detector simulation, $\left(X / X_{0}\right)^{M C}$, and installed in reality, $\left(X / X_{0}\right)^{d a t a}$, does not exceed the $3 \%$ level: $\Delta\left(X / X_{0}\right)=\left(X / X_{0}\right)^{\text {data }}-\left(X / X_{0}\right)^{M C}<0.0014$ with an average material thickness of $X / X_{0} \approx 0.045$.

The systematic uncertainty on the measured branching fraction was thus evaluated by comparing electron energy spectra in toy Monte Carlo simulations using three different material thicknesses, $X / X_{0}=0.0435, X / X_{0}=0.045$ (nominal) and $X / X_{0}=0.0465$. The electrons were intially generated with uniform angular distribution and energy distribution following the probability density functions (PDF's) $g\left(E_{e, 0}\right)$ for each relevant semileptonic $B$ decay channel: $B \rightarrow D, D^{*}, D^{* *}, D \pi$, and $X_{u} e \bar{\nu}_{e}$. The generated electrons were boosted into the lab frame, and electrons lying outside the geometrical detector acceptance were rejected. Each spectrum was then distorted by simulating bremsstrahlung emission. 

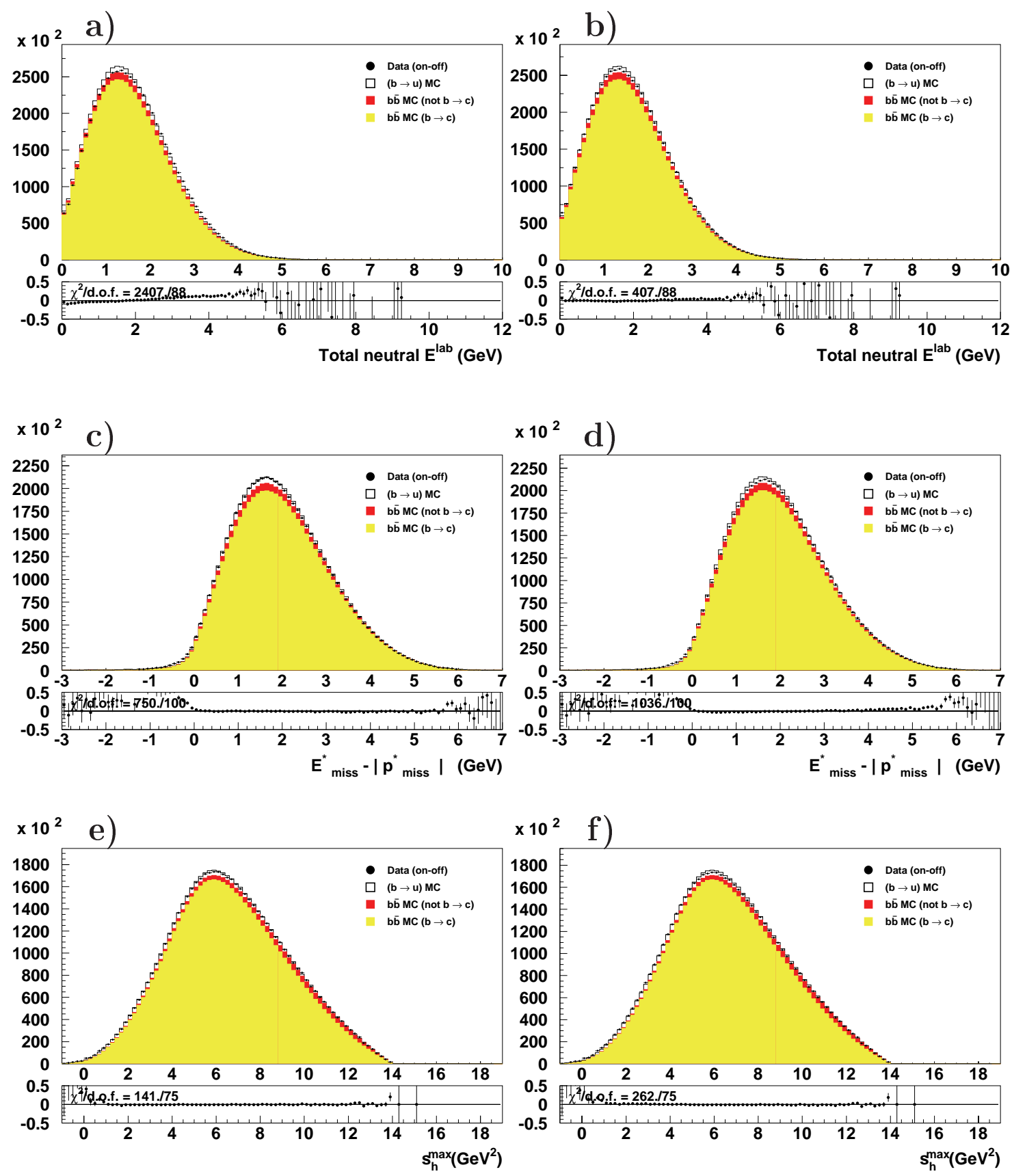

Figure 7.5: Effects of the uncertainty in the modeling of the $K_{L}^{0}$ energy deposition in terms of a) total neutral energy reconstruction, c) $E_{\text {miss }}^{*}-\left|\vec{P}_{\text {miss }}^{*}\right|$, and e) $s_{\mathrm{h}}^{\max }$ for a $-20 \%$ and $+20 \%$ efficiency change (b, d and f, respectively). These plots follow the same conventions as those of figure 5.2 . 
To do so, each detector volume was subdivided in thin slices of $\delta X_{i} / X_{0}$ such that $\sum_{i} \delta X_{i} / X_{0}=X / X_{0}$. For a given electron energy $E_{e, 0}$, a bremsstrahlung photon with a random energy $k_{i}$ was produced in the $i$ th detector slice according to the PDF

$$
f\left(k_{i}\right)=\frac{\delta X_{i}}{X_{0}} \cdot \frac{1}{k_{i}} \cdot\left[1-\frac{2}{3}\left(1-\frac{k_{i}}{E_{e, i}}\right)+\left(1-\frac{k_{i}}{E_{e, i}}\right)^{2}\right],
$$

where $E_{e, i}=E_{e, i-1}-k_{i-1}$ and $0<k_{i}<E_{e, i}[72]$. After having traversed the detector material the final electron energy was calculated as $E_{e, \text { final }}=E_{e, 0}-\sum_{i} k_{i}$. Finally, the resulting four-momentum vector was boosted back to the center-of-mass system. The procedure was repeated 50,000 times for each semileptonic $B$ decay channels to obtain the corresponding $E_{e, \text { final }}^{*}$ spectra. By comparing $E_{e, \text { final }}$ with $E_{e, 0}$, it was found that by applying an energydependent shift $\Delta E=\Delta E\left(E_{e}^{*}\right)$ on the spectrum for the default material thickness, one could effectively adjust the electron spectra for the above changes in the material thicknesses. This shift can approximately be described by $\Delta E=\mp 0.00125 E_{e}^{*}(\mathrm{GeV}) \pm 0.00425$ and holds for all semileptonic decay types $B \rightarrow D, D^{*}, D^{* *}, D \pi$, or $X_{u} e \bar{\nu}_{e}$.

The electron energy in the Monte Carlo simulation was then varied by this energy shift and the full set of kinematical variables used in the analysis such as $\left|\vec{P}_{\text {miss }}^{*}\right|, E_{\text {miss }}^{*}$ $\left|\vec{P}_{\mathrm{miss}}^{*}\right|, q^{2}$ and $s_{\mathrm{h}}^{\max }$ were recomputed. Since many photons have very low energies and closely follow the electron or positron, one must assume that not all of these photons are actually detected as visible neutral energy in the EMC. Therefore two extreme scenarios were considered:

1. None of the bremsstrahlung photons generated from thicker $(+)$ or thinner $(-)$ detector material were detected as neutral EMC clusters. As a consequence, $\left|\vec{P}_{\text {miss }}^{*}\right|$ and dependent variables were modified due to the change in the electron energy.

2. All bremsstrahlung photons generated from thicker $(+)$ or thinner $(-)$ detector material were detected as neutral EMC clusters, such that $\left|\vec{P}_{\text {miss }}^{*}\right|$ remained unchanged.

The resulting changes on the extraction parameters are outlined in table 7.6. Scenario 1) 

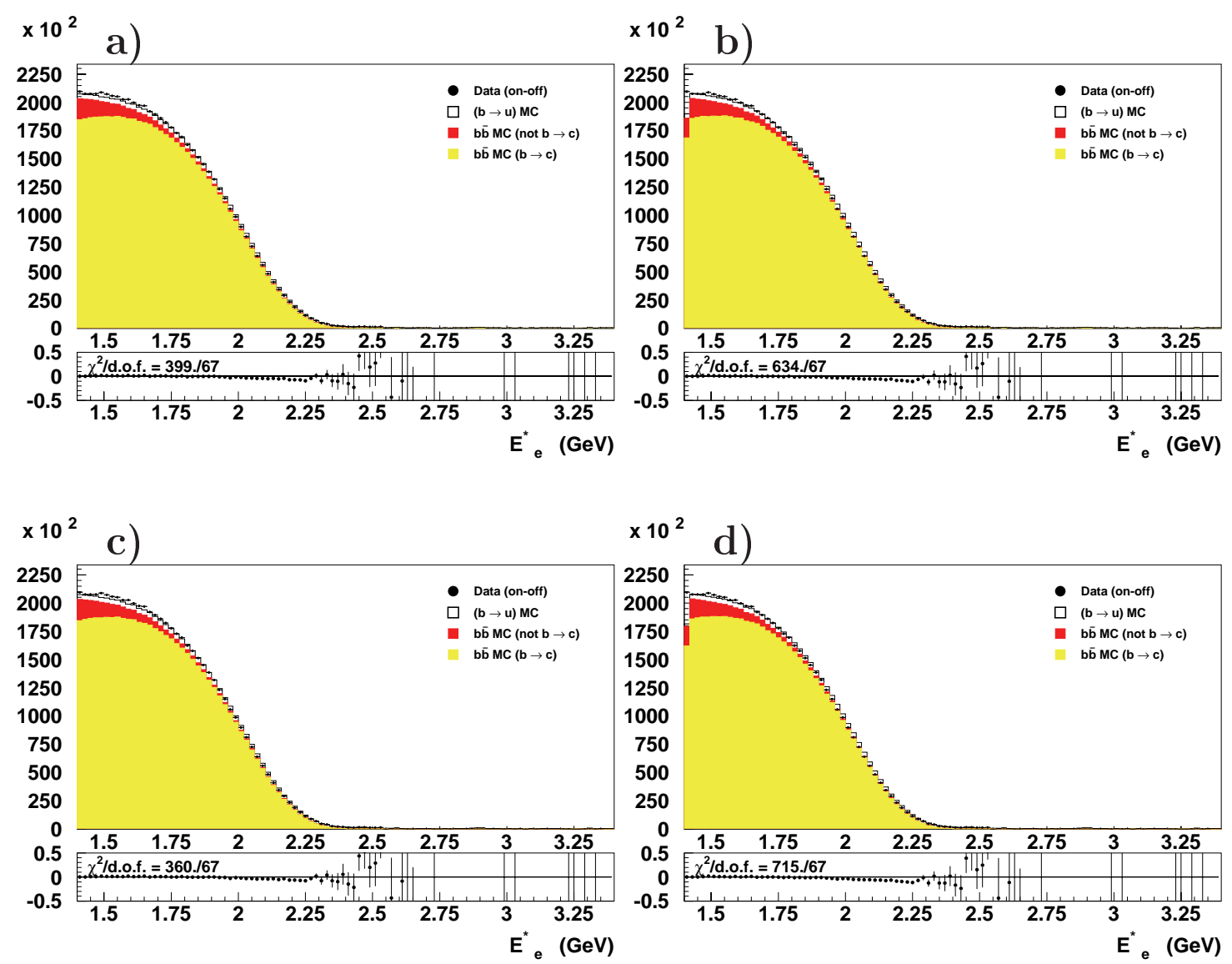

Figure 7.6: Effects of the uncertainty in simulating bremsstrahlung emission (top) and final state radiation (bottom) in terms of $E_{e}^{*}$. Shown are the 1 - (left) and $1+$ (right) cases. The above spectra should be compared with the nominal $E_{e}^{*}$ spectrum displayed in figure 5.8.

produced a larger variation than 2) and hence is taken as the bremsstrahlung systematic uncertainty.

\subsection{Radiative corrections}

Within the Monte Carlo simulation, final state radiation is modeled using the PHOTOS generator package [73]. To estimate the systematic uncertainty in the simulation results based using PHOTOS are compared with an analytical calculation for the radiative corrections provided by Ginsberg [74]. The decay $B \rightarrow D e^{-} \overline{\nu_{e}}$ was originally used to study the 


\begin{tabular}{|l|l|}
\hline Decay type & $\Delta E_{e}^{*}(\mathrm{MeV})$ \\
\hline \hline$B \rightarrow D e \bar{\nu}_{e}$ & $-2.0 E_{e}^{*}(\mathrm{GeV})+5.2$ \\
$B \rightarrow D^{*} e \bar{\nu}_{e}$ & $-2.0 E_{e}^{*}(\mathrm{GeV})+5.2$ \\
$B \rightarrow D \pi e \bar{\nu}_{e}$ & $-1.5 E_{e}^{*}(\mathrm{GeV})+4.0$ \\
$B \rightarrow D^{* *} e \bar{\nu}_{e}$ & $-5.0 E_{e}^{*}(\mathrm{GeV})+1.7$ \\
$B \rightarrow X_{u} e \bar{\nu}_{e}$ & $-0.7 E_{e}^{*}(\mathrm{GeV})+17$ \\
\hline
\end{tabular}

Table 7.8: Final state radiation uncertainty and resulting $E_{e}^{*}$ for various semileptonic $B$ meson decays.

performance of the PHOTOS generator by measuring the quantity

$$
\frac{\Delta \Gamma}{d x}-\frac{\Delta \Gamma_{0}}{d x}
$$

where $\Delta \Gamma / d x$ is the lepton energy spectrum corrected for single photon emission, $\Delta \Gamma_{0} / d x$ is the lepton energy spectrum without radiative corrections and $x=E_{e} / E_{\max }$, and comparing results with the analytical calculations. For each semileptonic decay type, $B \rightarrow$ $D, D^{*}, D^{* *}, D \pi$, and $X_{u} e \bar{\nu}_{e}$, equation 7.9 was evaluated using the PHOTOS and Ginsberg approach from the electron energy spectrum in the simulation. The difference

$$
\left[\frac{\Delta \Gamma}{d x} \mid \text { Ginsberg }-\frac{\Delta \Gamma_{0}}{d x}\right]-\left[\left.\frac{\Delta \Gamma}{d x}\right|_{\text {РнотоS }}-\frac{\Delta \Gamma_{0}}{d x}\right]=\frac{\Delta \Gamma}{d x} \mid \text { Ginsberg }-\left.\frac{\Delta \Gamma}{d x}\right|_{\text {РнотоS }}
$$

was translated into an electron energy shift between the PHOTOS and Ginsberg spectrum. This shift was found to be different for the various semileptonic $B$-decay types and the results are listed in table 7.8. The $\pm \Delta E_{e}^{*}$ shift is therefore taken as the uncertainty from the final state radiation.

As in the case of bremsstrahlung, two extreme scenarios are considered to evaluate the systematic uncertainties on $\mathcal{B}\left(B \rightarrow X_{u} e \bar{\nu}_{e}\right)$ :

1. The photon from the final state radiation $( \pm)$ is not detected. As a consequence, $\left|\vec{P}_{\text {miss }}^{*}\right|$ and dependent variables are modified due to the change in the electron energy.

2. The photon from the final state radiation $( \pm)$ is detected as a separate neutral EMC cluster such that $\left|\vec{P}_{\text {miss }}^{*}\right|$ remains unchanged.. 
As shown in table 7.6, scenario 1) yields the larger variation and is therefore taken as the systematic uncertainty due to the simulation of the final state radiation.

\subsection{3 $B$-meson counting and continuum scaling}

The techniques used in extracting the luminosity and $N_{B \bar{B}}$ are described in detail in [75] and [76]. Accordingly, the number of $B \bar{B}$ pairs is evaluated using [76] and yields $(88.35 \pm$ $0.97) \times 10^{6}$. Although the uncertainty on $N_{B \bar{B}}$ is evaluated to be $1.1 \%$, the relative uncertainty between on-peak and off-peak luminosity is only $0.25 \%$. Since $1867 \pm 125$ off-peak events are observed to be within the signal region, this corresponds to an additional systematics of \pm 4.7 events. This uncertainty is negligible with respect to the statistical uncertainty and is ignored. If the energy scaling of off-peak data to on-peak energy is not performed, the off-peak contribution in the signal region decreases by 8 events. The uncertainty on the energy scaling is therefore negligible. Therefore, a $1.1 \%$ uncertainty on $\mathcal{B}_{u}^{\mathrm{SL}} \Delta \mathcal{B}_{u}^{\mathrm{SL}}$ due to the measurement of $N_{B \bar{B}}$.

\subsection{Summary of uncertainties}

Table 7.9 summarizes the dominant systematic uncertainties associated with the measurement of the $B \rightarrow X_{u} e \bar{\nu}_{e}$ branching ratio. Due to the technique used, some of the systematics could only be evaluated in one direction $(+$ or -$)$. For instance, it was possible to apply track killing to the simulation, but new tracks cannot be created. Therefore, for the tracking efficiency, neutrals reconstruction and charged particle ID, the uncertainties are symmetrized. Furthermore, in the case where uncertainties were evaluated in both direction but yielded different values, the largest discrepancy ( + or -$)$ was taken as the systematic error $( \pm)$. This results in a systematic uncertainty from detector modeling of $5.9 \%$ on $\mathcal{B}_{u}^{\text {SL }}$ to add in quadrature with the $\pm 5.9 \%$ systematic effect observed from background uncertainties and $10.8 \%$ from the modeling of the signal $B \rightarrow X_{u} e \bar{\nu}_{e}$ simulation. Therefore, the total 


\begin{tabular}{|l|c|c|}
\hline Source of Systematics & $\sigma_{\mathcal{B}_{u}^{\mathrm{SL}}}(\%)$ & $\sigma_{\Delta \mathcal{B}_{u}^{\mathrm{SL}}}(\%)$ \\
\hline \hline 1a) Tracking efficiency & 1.4 & 1.3 \\
\hline 2a) Electron ID efficiency & 0.9 & 0.9 \\
\hline 3a) Charged particle ID & 2.5 & 2.6 \\
\hline 4a) Neutrals reconstruction & 3.3 & 3.1 \\
\hline 5a) $K_{L}^{0}$ energy deposition & 3.3 & 3.1 \\
\hline 6a) Bremsstrahlung & 1.8 & 2.3 \\
\hline 7a) B counting & 1.1 & 1.1 \\
\hline \hline A) Detector modeling & 5.9 & 5.9 \\
\hline \hline 1b) $B \rightarrow X_{c} \ell \nu$ simulation & 5.0 & 5.0 \\
\hline 2b) Radiative corrections & 3.2 & 3.6 \\
\hline \hline B) Background simulation & 5.9 & 6.2 \\
\hline \hline 1c) $B \rightarrow X_{u} \ell \nu$ simulation & 4.0 & 4.4 \\
\hline 2c) Shape function uncertainties & 10.0 & 1.3 \\
\hline \hline C) Signal simulation & 10.8 & 4.6 \\
\hline \hline Total $(A \oplus B \oplus C)$ & 13.6 & 9.7 \\
\hline
\end{tabular}

Table 7.9: Summary of systematic uncertainties. The systematics on the signal simulation also include the theoretical uncertainties associated with the uncertainty on the HQE parameters $m_{b}$ and $a$. The total systematical uncertainty was computed as the quadratic sum of the uncertainties. In the case where a variation was taken only in one direction, the corresponding uncertainty was symmetrized.

systematic uncertainty on the measurement of the inclusive $B \rightarrow X_{u} e \bar{\nu}_{e}$ branching ratio is

$$
\sigma_{\mathcal{B}_{u}^{S \mathrm{~L}}}=5.9 \%_{(\mathrm{det})} \pm 5.9 \%_{(\mathrm{bkg})} \pm 10.8 \%_{(\mathrm{sig})}= \pm 13.6 \%
$$

Similar uncertainties were obtained on the partial branching ratio and yield

$$
\sigma_{\Delta \mathcal{B}_{u}^{\mathrm{SL}}}=5.9 \%_{(\mathrm{det})} \pm 6.2 \%(\mathrm{bkg}) \pm 4.6 \%(\mathrm{sig})= \pm 9.7 \% \text {. }
$$

As expected, the unfolding procedure reduces the systematics due to HQE parameter uncertainties in signal modeling. The uncertainties were also evaluated for different requirements on the $E_{e}^{*}$. The overall uncertainty on $\mathcal{B}_{u}^{\mathrm{SL}}$ is minimal for $E_{e}^{*}>2.1 \mathrm{GeV}$, although similar results were obtained at 2.0 and $2.2 \mathrm{GeV}$, as shown in appendix $\mathrm{C}$. 


\section{Chapter 8}

\section{Results}

Varium et mutabile...

- Virgil

\subsection{Measurements of $\mathcal{B}_{u}^{\mathrm{SL}}$ and $\Delta \mathcal{B}_{u}^{\mathrm{SL}}$}

Since the dominant systematic uncertainties have been evaluated, the (partial) $B \rightarrow X_{u} e \bar{\nu}_{e}$ branching ratio may now be extracted in the signal region using the procedure described in section 7.1. A summary of the yields and efficiencies obtained for both data and Monte Carlo simulation is provided in table 8.1. Using equation 7.1 and the uncertainties summarized in table 7.9 , the inclusive $B \rightarrow X_{u} e \bar{\nu}_{e}$ branching ratio is measured to be

$$
\mathcal{B}_{u}^{\mathrm{SL}}=\left(2.54 \pm 0.24_{(\text {stat })} \pm 0.35_{(\text {syst })}\right) \times 10^{-3}
$$

\begin{tabular}{|l|l||l|l|}
\hline \multicolumn{2}{|c|}{ Inputs from data } & \multicolumn{2}{|c|}{ Inputs from MC } \\
\hline \hline$N_{\text {cand }}^{\text {data }}$ & $5130 \pm 150$ & $M_{b k g}^{M C}$ & $3176 \pm 35$ \\
$N_{\text {side }}^{\text {data }}$ & $6152 \pm 130$ & $M_{\text {side }}^{M C}$ & $6423 \pm 49$ \\
$\epsilon_{\text {dee }}^{\text {data }}$ & $(90.0 \pm 3.9) \times 10^{-3}$ & $\epsilon_{D e \nu}^{\mathrm{MC}}$ & $(90.5 \pm 1.9) \times 10^{-3}$ \\
& & $\epsilon_{u}^{\mathrm{MC}}$ & $(4.765 \pm 0.050) \times 10^{-3}$ \\
& & $f_{u}$ & $0.1401 \pm 0.0004$ \\
& & $\epsilon_{\text {sig }}$ & $(30.1 \pm 0.3) \times 10^{-3}$ \\
& & $\epsilon_{\overline{\mathrm{sig}}}$ & $(0.558 \pm 0.021) \times 10^{-3}$ \\
\hline
\end{tabular}

Table 8.1: Data and Monte Carlo yields and efficiencies used for computing signal branching ratio. The uncertainties are purely statistical. 

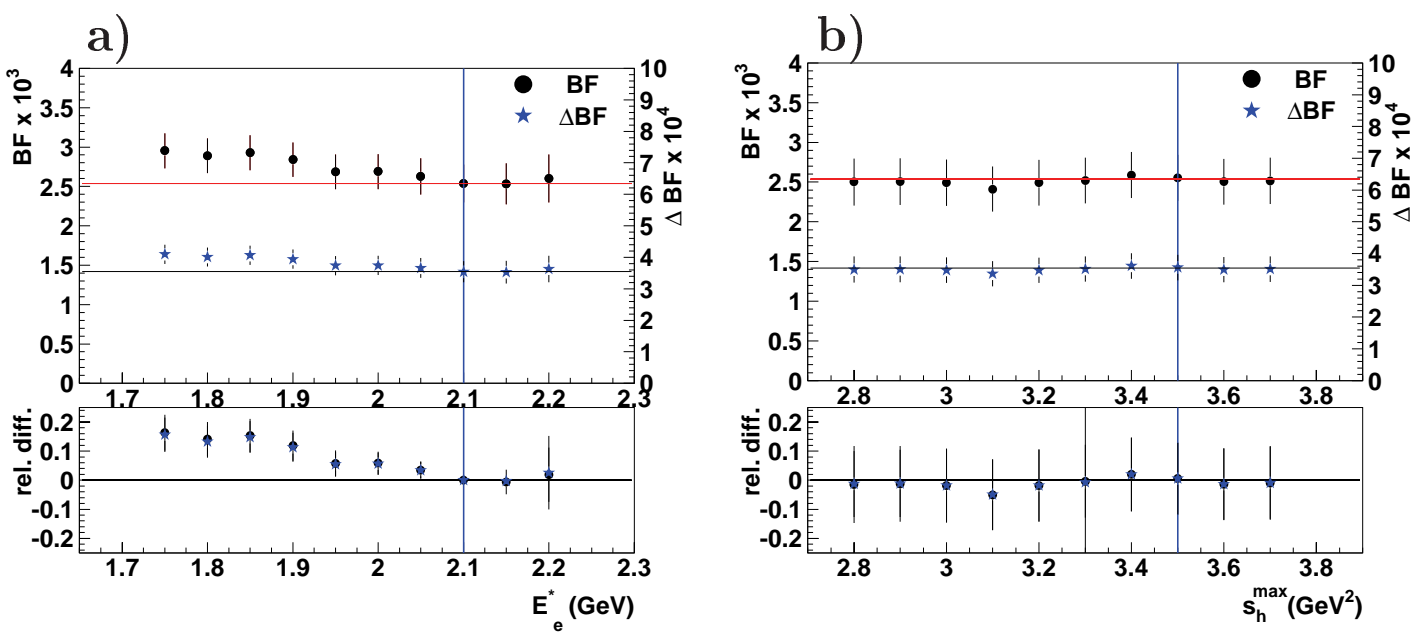

Figure 8.1: Changes in the $B \rightarrow X_{u} e \bar{\nu}_{e}$ branching fraction measurement (solid points) as a function of a) the lower bound on $E_{e}^{*}$ and b) the upper bound on $s_{\mathrm{h}}^{\max }$. The behavior of the partial branching ratio is also displayed (stars) and the appropriate scale is provided on the right hand side of the plots. The lower plots display the relative difference with respect to the nominal requirements: $\mathcal{B}_{u}^{\mathrm{SL}}\left(E_{e}^{*}>x\right) / \mathcal{B}_{u}^{\mathrm{SL}}\left(E_{e}^{*}>2.1 \mathrm{GeV}\right)-1$, and $\Delta \mathcal{B}_{u}^{\mathrm{SL}}\left(E_{e}^{*}>\right.$ $x) / \Delta \mathcal{B}_{u}^{\mathrm{SL}}\left(E_{e}^{*}>2.1 \mathrm{GeV}\right)-1$. The errors shown are purely statistical.

where the above errors correspond to the total statistical and systematic uncertainties, respectively. Similarly, the unfolded partial branching ratio is evaluated using equation 7.6 to be

$$
\Delta \mathcal{B}_{u}^{\mathrm{SL}}\left(\tilde{E}_{e}, s_{\mathrm{h}}^{\max }\right)=\left(3.54 \pm 0.33_{(\text {stat })} \pm 0.34_{(\text {syst })}\right) \times 10^{-4}
$$

for the portion of the phase-space contained within $E_{e}^{0}>2.0 \mathrm{GeV}$ and $s_{\mathrm{h}}^{\max }<3.5 \mathrm{GeV}^{2}$.

The stability in extracting $\mathcal{B}_{u}^{\mathrm{SL}}$ and $\Delta \mathcal{B}_{u}^{\mathrm{SL}}$ as a function of the important analysis requirements was considered; the results are shown in figures 8.1 and 8.3. In these figures, the upper plots show the absolute measurements for the given requirements, whereas the lower plots display the relative difference with respect to the nominal values, i.e.

$$
\frac{\mathcal{B}_{u}^{\mathrm{SL}}(\text { cut }=\mathrm{x})}{\mathcal{B}_{u}^{\mathrm{SL}}(\text { nomi })}-1 \text { and } \frac{\Delta \mathcal{B}_{u}^{\mathrm{SL}}(\text { cut }=\mathrm{x})}{\Delta \mathcal{B}_{u}^{\mathrm{SL}}(\text { nomi })}-1 .
$$

All of the errors shown are statistical, and in the case of the relative difference, the errors were computed from the residual difference in the yields with respect to the nominal values. 


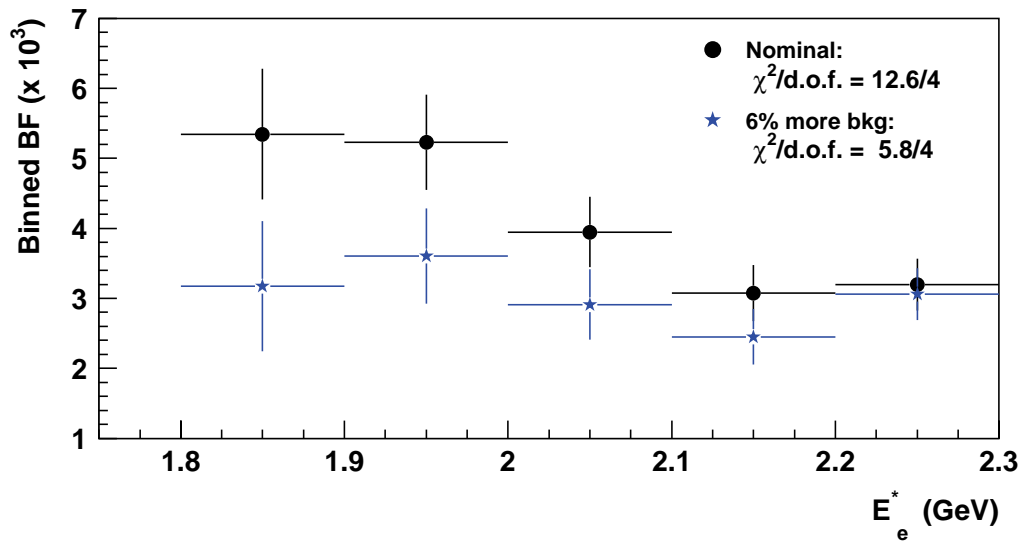

Figure 8.2: The branching fraction evaluated in bins of $E_{e}^{*}$ is shown for the nominal background (solid points) and for a $6 \%$ increase in the background (stars). The errors shown are purely statistical. Hence, the apparent discrepancy observed in the $\mathcal{B}_{u}^{\mathrm{SL}}$ extraction as a function of the lower bond on $E_{e}^{*}$ is within the background systematic uncertainties listed in table 7.9 .

The scans versus $E_{e}^{*}$ and $s_{\mathrm{h}}^{\max }$ are sensitive to mis-modeling of backgrounds and also to the modeling of $B \rightarrow X_{u} e \bar{\nu}_{e}$ decays. The $E_{e}^{*}$ scan suggests that the background modeling is imperfect. In fact, the variation seen between $1.9 \mathrm{GeV}$ and $2.1 \mathrm{GeV}$ is too large to be attributed to signal modeling; it is the background fraction that is changing rapidly in this region. Since this analysis is dominated by systematic uncertainties, a proper understanding of this scan requires a calculation of the systematics at each scan point. Given the complexity of interpreting the scan deviations and uncertainties, a set of branching fraction values determined in distinct bins of $E_{e}^{*}$ were computed and are shown in figure 8.2. The solid points are the results obtained with the nominal background estimates, and the uncertainties are purely statistical. Also displayed are the results when the background is increased by $6 \%$, which corresponds to the systematic uncertainties assigned to the modeling of the background (see table 7.9). The variation observed in the branching fraction as a function of $E_{e}^{*}$ is clearly consistent with the systematic uncertainty assigned to the background: a 
$\chi^{2} /$ d.o.f. consistency test was performed and yield 5.8/4. Hence, no additional systematic uncertainties are required to account for the $E_{e}^{*}$ behavior.

Scans taken as functions of other kinematical requirements show only small variations. The $E_{\text {miss }}^{*}-\left|\vec{P}_{\text {miss }}^{*}\right|$ scan is sensitive to the simulation of the missing energy-momentum reconstruction, as is the scan versus $\left|\vec{P}_{\text {miss }}^{*}\right|$; as mentioned in section 5.4, events with $\left|\vec{P}_{\text {miss }}^{*}\right|>$ $2.1 \mathrm{GeV}$ carry little information about the true neutrino momentum. While the optimization scans show only a small improvement from removing events with $\left|\vec{P}_{\text {miss }}^{*}\right|>2.5 \mathrm{GeV}$, the scan shows whether the data and Monte Carlo simulations are consistent in this region.

\subsection{Classic extraction of $\left|V_{u b}\right|$}

Given the measured branching fraction, one can determine $\left|V_{u b}\right|$ by comparing with theoretical predictions $[26,34,36,37]$. If the inclusive branching fraction is used to extract the total charmless semileptonic decay width $\Gamma\left(B \rightarrow X_{u} e \bar{\nu}_{e}\right)$, the relation

$$
\left|V_{u b}\right|=0.00424 \sqrt{\frac{\mathcal{B}\left(B \rightarrow X_{u} e \bar{\nu}_{e}\right)}{0.002} \frac{1.61 \mathrm{ps}}{\tau_{B}}}(1 \pm 0.048)
$$

described in section 2.5.5 can be used to determine $\left|V_{u b}\right|$ with modest theoretical uncertainty. Incorporating the measurement of the branching ratio and the world average $B$-lifetime measurement ${ }^{1}$ into equation 2.35 , a measurement of

$$
\left|V_{u b}\right|=\left(4.79 \pm 0.40_{(\text {expt })} \pm 0.23_{\left(\mathcal{B}_{u}^{\mathrm{SL}} \rightarrow\left|V_{u b}\right|\right)}\right) \times 10^{-3}
$$

is obtained, where the errors correspond to experimental and theoretical uncertainties, respectively. As discussed in appendix $\mathrm{C}$, alternative $\mathcal{B}_{u}^{\mathrm{SL}}$ measurements were performed for different $E_{e}^{*}$ requirements and yield

$$
\left|V_{u b}\right|=\left(4.93 \pm 0.44_{(\text {expt })} \pm 0.24_{\left(\mathcal{B}_{u}^{\mathrm{SL}} \rightarrow\left|V_{u b}\right|\right)}\right) \times 10^{-3}
$$

for $E_{e}^{*}>2.0 \mathrm{GeV}$, and

$$
\left|V_{u b}\right|=\left(4.84 \pm 0.45_{(\operatorname{expt})} \pm 0.24_{\left(\mathcal{B}_{u}^{\mathrm{SL}} \rightarrow\left|V_{u b}\right|\right)}\right) \times 10^{-3} .
$$

\footnotetext{
${ }^{1}$ From the average of $\tau_{B^{0}}=(1.536 \pm 0.014)$ ps and $\tau_{B^{+}}=(1.671 \pm 0.018)$ ps.
} 
a)

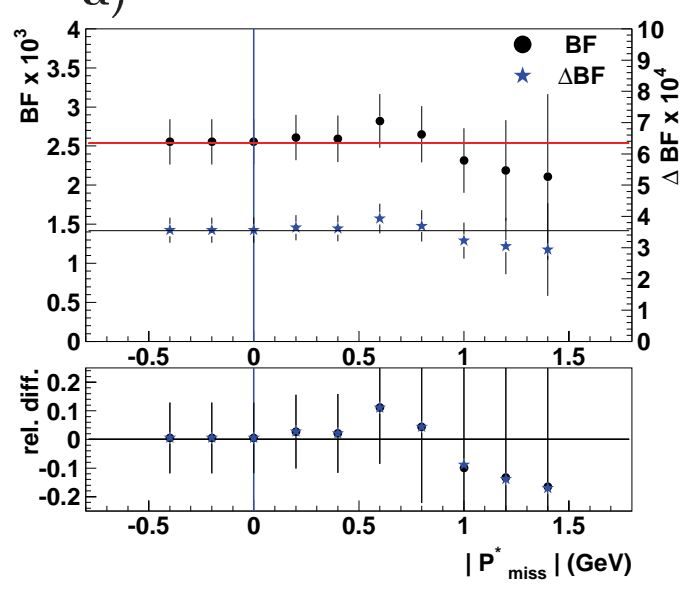

c)

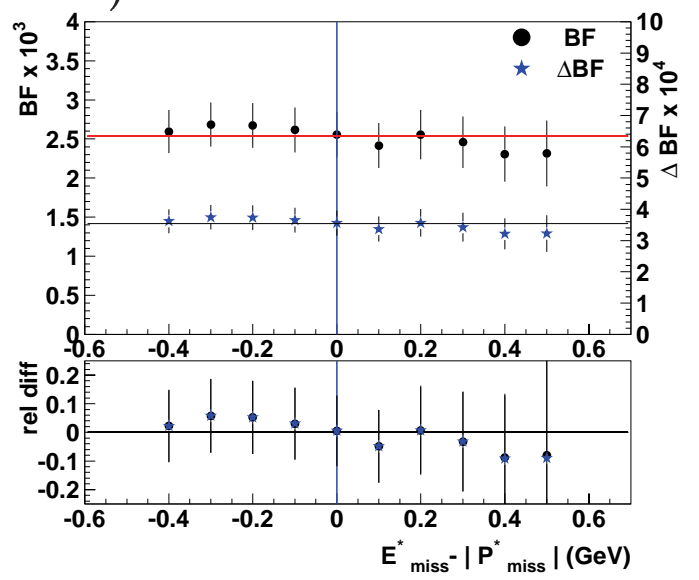

e)

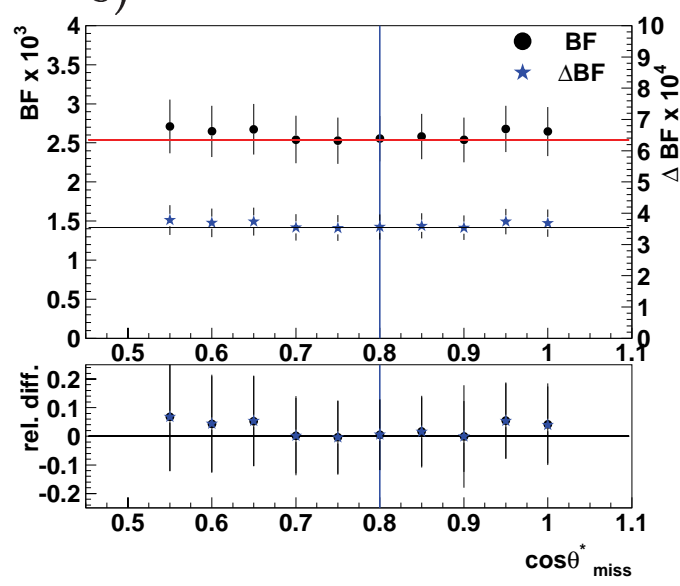

b)

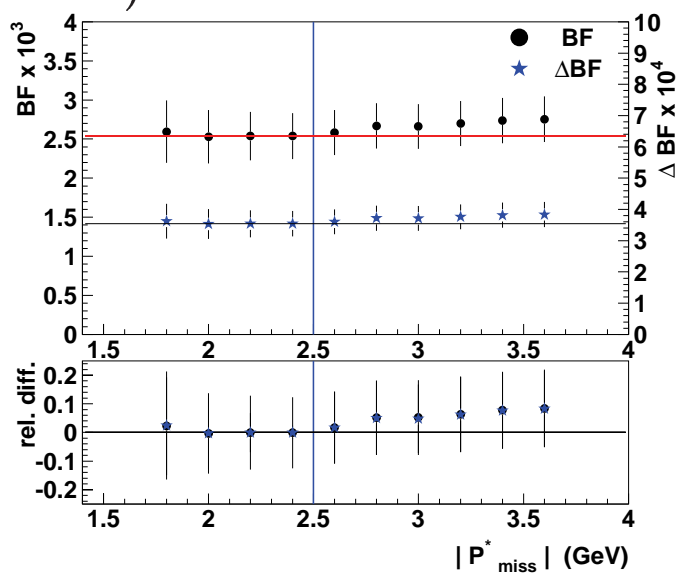

d)

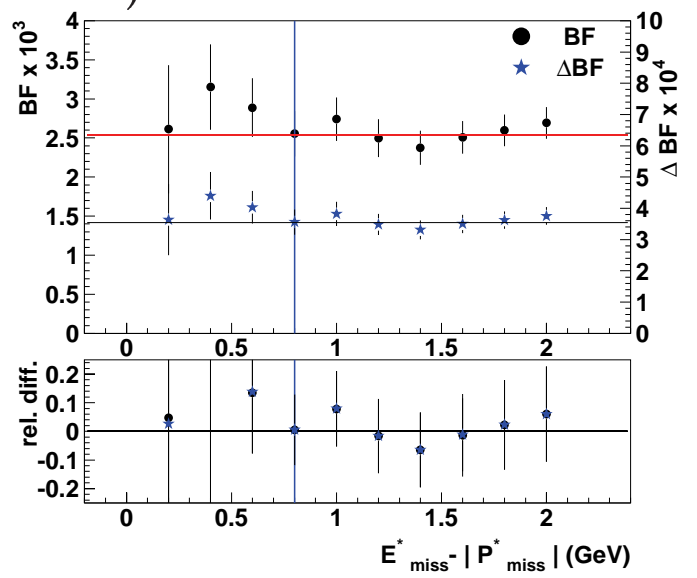

f)

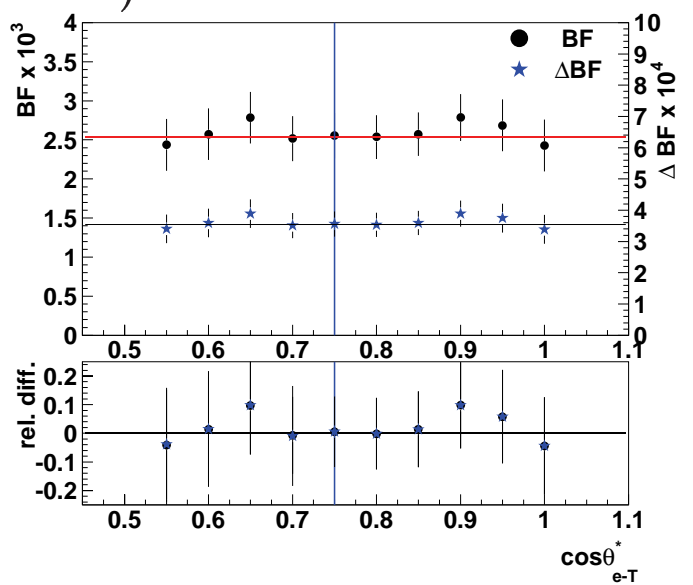

Figure 8.3: Changes in the $B \rightarrow X_{u} e \bar{\nu}_{e}$ branching fraction and partial branching ratio measurements as a function of a) the lower and b) upper bound on $\left|\vec{P}_{\text {miss }}^{*}\right|$, c) the lower and d) upper bound on $E_{\text {miss }}^{*}-\left|\vec{P}_{\text {miss }}^{*}\right|$, e) the upper bound on $\cos \theta_{\text {miss }}^{*}$, and f) the bound on $\cos \theta_{\vec{e} \cdot \vec{T}}^{*}$. These plots follow the same convention as those of figure 8.1. 
for $E_{e}^{*}>2.2 \mathrm{GeV}$.

The above results for $\left|V_{u b}\right|$ are in good agreement for the various $E_{e}^{*}$ requirements, and in good agreement with the world average of $(4.70 \pm 0.44 \pm 0.23) \times 10^{-3}$ [19]. These results are based upon original HQE calculations performed to order $1 / m_{b}^{2}$ including order $\alpha_{s}$ corrections [38], but recent theoretical results including higher order corrections have appeared in the litterature [77], and the evaluated ratio of the partial branching fraction to the total charmless semileptonic branching fraction $\left(f_{u}\right)$ has changed dramatically, mostly due to changes in the HQE parameters. Results based on these higher order calculations are presented in the following section.

\subsection{Improved theory and $\left|V_{u b}\right|$ extraction}

The new theoretical computations have recently become available in which the leading terms in the HQE of the $B \rightarrow X_{s} \gamma$ and $B \rightarrow X_{u} e \bar{\nu}_{e}$ spectra are computed at next-to-leading order, and power corrections are included at $\mathcal{O}\left(\alpha_{s}\right)$ for the leading SF and at tree level for subleading SFs [77]. The values used for the heavy quark parameters, $m_{b}=4.61 \pm 0.08 \mathrm{GeV}$ and $\mu_{\pi}^{2}=0.15 \pm 0.07 \mathrm{GeV}$ are based on fits to $B \rightarrow X_{c} e \bar{\nu}_{e}$ moments [25], translated to the shape-function scheme [78].

In this new context, $\left|V_{u b}\right|$ is extracted from the partial rate, $\Delta \Gamma=\Delta \mathcal{B}_{u}^{\mathrm{SL}} / \tau_{B}$, as

$$
\begin{aligned}
\left|V_{u b}\right| & =\left[\frac{\Delta \Gamma}{\Delta \zeta}\right]^{1 / 2} \\
& =\left[\frac{\Delta \mathcal{B}_{u}^{\mathrm{SL}}}{\Delta \zeta \tau_{B}}\right]^{1 / 2},
\end{aligned}
$$

where $\Delta \zeta$ is the normalized theoretical rate, $\Delta \Gamma /\left|V_{u b}\right|^{2}$. The theoretical rate for $s_{\mathrm{h}}^{\max }<$ $3.5 \mathrm{GeV}^{2}$ and corresponding heavy quark parameter uncertainties are listed in table 8.2. Also outlined are the theoretical uncertainties due to subleading SFs [79], weak annihilation [80] and variations in the matching scales used in the calculation.

After substituting the unfolded partial branching ratio obtained above into equa- 


\begin{tabular}{|c|c|c|c|}
\hline Requirement & $\Delta \zeta\left(\mathrm{ps}^{-1}\right)$ & $\sigma_{\mathrm{HQE}}(\%)$ & $\sigma_{\text {theo }}(\%)$ \\
\hline \hline$\tilde{E}_{e}>1.9 \mathrm{GeV}$ & 16.09 & ${ }_{-20.8}^{+25.9}$ & \pm 11.8 \\
& & ${ }_{-21.1}$ & \pm 12.6 \\
$\tilde{E}_{e}>2.0 \mathrm{GeV}$ & 14.19 & ${ }_{-28.2}$ & \\
& & ${ }_{-25.3}^{+34.2}$ & \pm 14.7 \\
$\tilde{E}_{e}>2.1 \mathrm{GeV}$ & 11.09 & \\
\hline
\end{tabular}

Table 8.2: Theoretical rates for various $E_{e}^{0}$ requirements and associated theoretical uncertainties [77]. The SF uncertainty is computed at tree level and depends on the HQE parameter $m_{b}$ and $\mu_{\pi}^{2}$, whereas the theoretical error contains uncertainties from subleading SFs and weak annihilation.

tion $8.3,\left|V_{u b}\right|$ is determined to be

$$
\left|V_{u b}\right|=\left(3.95 \pm 0.27_{(\text {expt })}{ }_{-0.42}^{+0.58} \text { (HQE) } \pm 0.25_{(\text {theo })}\right) \times 10^{-3}
$$

where the first error is experimental (from $\Delta \mathcal{B}_{u}^{\mathrm{SL}}$ ), the second due to heavy quark parameters uncertainties and the third due to theory. No uncertainty is assigned for possible quarkhadron duality violation.

Similar results are obtained from the measurements of the partial branching fraction for two different $\tilde{E}_{e}$ cuts:

$$
\left|V_{u b}\right|=\left(4.07 \pm 0.32_{(\text {expt })}^{+0.53}{ }_{-0.42(\mathrm{HQE})}^{\left.+0.24_{(\text {theo })}\right) \times 10^{-3}}\right.
$$

for $\tilde{E}_{e}>1.9 \mathrm{GeV}$, and

$$
\left|V_{u b}\right|=\left(4.09 \pm 0.31_{(\mathrm{expt})}{ }_{-0.52(\mathrm{HQE})}^{+0.70} \pm 0.30_{(\text {theo })}\right) \times 10^{-3}
$$

for $\tilde{E}_{e}>2.1 \mathrm{GeV}$ (see appendix C). These results are consistent for all three different $E_{e}^{0}$ requirements, but differ from the $\left|V_{u b}\right|$ values obtained in section 8.2 , mostly because of the changes in the interpretation of the HQE parameters.

The determination of $\left|V_{u b}\right|$ is limited primarily by our knowledge of the $b$-quark mass. Otherwise, the sensitivity to higher moments of the SF is weak. In fact, the change in $\left|V_{u b}\right|$ when varying $-\lambda_{1}$ from 0.03 to $0.35 \mathrm{GeV}$ with $m_{b}$ fixed is $2 \%$, and the impact of using alternative SF parameterizations is $<2 \%$ (see section 7.3 ). 


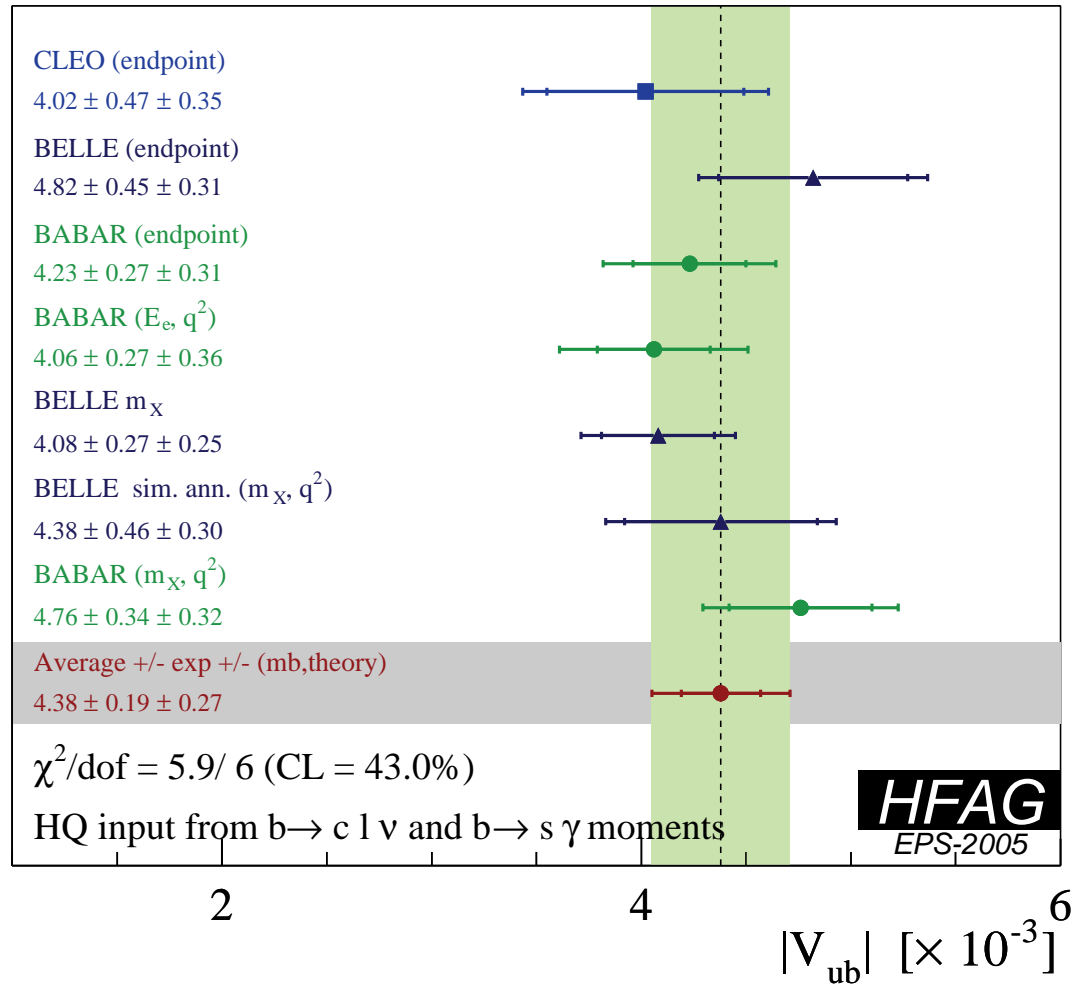

Figure 8.4: Comparison between inclusive $\left|V_{u b}\right|$ determinations as presented at the 2005 European Physical Society meeting [19]. In order to compare the various results, the heavy quark parameters input are set using a global fit to both the $B \rightarrow X_{c} \ell \bar{\nu}_{\ell}$ and $B \rightarrow X_{s} \gamma$ which yields $m_{b}=4.60 \pm 0.04 \mathrm{GeV}$ and $-\lambda_{1}=0.20 \pm 0.04 \mathrm{GeV}^{2}$.

Shown in figure 8.4 are the results from recent inclusive $\left|V_{u b}\right|$ determinations based on the $B \rightarrow X_{u} e \bar{\nu}_{e}$ model described in Ref. [77]. In order to compare the various results, the heavy quark parameters input are set using a global fit to both the $B \rightarrow X_{c} \ell \bar{\nu}_{\ell}$ and $B \rightarrow X_{s} \gamma$ which yields $m_{b}=4.60 \pm 0.04 \mathrm{GeV}$ and $-\lambda_{1}=0.20 \pm 0.04 \mathrm{GeV}^{2}$ [19]. This results into much smaller SF uncertainties for all of the analyses. The overall precision on $\left|V_{u b}\right|$ from this work is comparable to determinations of $\left|V_{u b}\right|$ which have become available while this study was nearing completion [81]. In fact, the uncertainty on $\left|V_{u b}\right|$ from the hadronic mass and endpoint analyses have significantly improved with respect to the results shown in figure 2.8. The reason for this is two-fold: the precision on measurements of the $b$ quark mass has much improved, and the endpoint analyses have lowered their cut down 
to $2 \mathrm{GeV} / c$. Non-factorizable effects such as subleading SFs and weak annihilation lead to larger theoretical uncertainties in the $q^{2}-E_{\ell}$ approach due to the portion of the $B \rightarrow X_{u} \ell \bar{\nu}_{\ell}$ phase-space probed. Future measurements of the separate charmless semileptonic branching fractions for charged and neutral $B$ mesons may soon reduce this uncertainty.

\subsection{Outlook}

Currently, the precision in the extraction of the CKM element $\left|V_{u b}\right|$ is primarily limited by the uncertainties on the heavy quark parameters, in particular on the $b$-quark mass. The study of the $E_{\gamma}$ moments in $B \rightarrow X_{s} \gamma$ decays at $B A B A R$ will soon become available and should improve our current knowledge of $m_{b}$. Theoretical calculations are also being developed to allow $B \rightarrow X_{s} \gamma$ studies to be combined with $B \rightarrow X_{c} \ell \bar{\nu}_{\ell}$ moment analyses and should further improve the precision on HQE parameters. Such calculations were used in generating the results shown in figure 8.4, and result in 50\% smaller uncertainties due to the heavy quark parameters. Alternative calculations which allow the extraction of $\left|V_{u b}\right|$ from inclusive $B \rightarrow X_{u} \ell \bar{\nu}_{\ell}$ decays without recourse of shape functions using the lepton energy endpoint [82] and the hadronic mass spectrum [83] are also being considered. Extraction of $\left|V_{u b}\right|$ based on these calculations are currently being reviewed and should soon become available [84]. Furthermore, the separate studies of neutral and charged $B$ meson decays will put constraints on higher order processes such as weak annihilation and theoretical uncertainties will be reduced. Finally, improvements in lattice QCD and LCSR calculations may provide much better form-factor predictions and allow the extraction of $\left|V_{u b}\right|$ for some exclusive decay channels, in particular $B^{0} \rightarrow \pi^{+} \ell^{-} \bar{\nu}_{\ell}$, with much better uncertainties $[24,85]$. This would be a valuable since the theoretical models involved are independent of each other. 


\section{Chapter 9}

\section{Conclusion}

Sic transit gloria mundi

In this dissertation, a measurement of the CKM matrix element $\left|V_{u b}\right|$ was performed based on the study of 88 million $B \bar{B}$ pairs collected by $B A B A R$ detector. Inclusive semileptonic $B \rightarrow X_{u} e \bar{\nu}_{e}$ decays were identified using a novel approach based on simultaneous requirements for the electron energy, $E_{e}$, and the invariant mass squared of the $e \bar{\nu}_{e}$ pair, $q^{2}$. The neutrino 4-momentum was reconstructed from the visible 4-momentum and knowledge of the $e^{+} e^{-}$initial state. The dominant charm background was suppressed by selecting a region of the $q^{2}-E_{e}$ phase space where correctly reconstructed $B \rightarrow X_{c} e \bar{\nu}_{e}$ events were kinematically excluded. Background contamination in the signal region due to resolution effects was evaluated in Monte Carlo simulations. The partial $B \rightarrow X_{u} e \bar{\nu}_{e}$ was measured to be $\Delta \mathcal{B}_{u}^{\mathrm{SL}}\left(\tilde{E}_{e}>2.0 \mathrm{GeV}, s_{\mathrm{h}}^{\max }<3.5 \mathrm{GeV}^{2}\right)=\left(3.54 \pm 0.33_{\text {(stat) }} \pm 0.34_{(\text {syst })}\right) \times 10^{-4}$ based on the observed yield in data, and background estimates and signal efficiencies evaluated in Monte Carlo simulations.

The extraction of $\left|V_{u b}\right|=\left[\Delta \mathcal{B} /\left(\Delta \zeta \tau_{B}\right)\right]^{1 / 2}$ was based on recent theoretical calculations [77], in which the leading terms in the HQE of the $B \rightarrow X_{u} e \bar{\nu}_{e}$ spectra are computed at next-to-leading order, and power corrections are included at $\mathcal{O}\left(\alpha_{S}\right)$ for the leading SF and at tree level for subleading SFs. The values used for the heavy quark parameters, $m_{b}=4.61 \pm 0.08 \mathrm{GeV}$ and $\mu_{\pi}^{2}=0.15 \pm 0.07 \mathrm{GeV}$, are based on fits to $B \rightarrow X_{c} \ell \bar{\nu}_{\ell}$ moments [25], translated to the shape-function scheme [78]. 
A value of $\left|V_{u b}\right|=\left(3.95 \pm 0.27_{-0.42}^{+0.58} \pm 0.25\right) \times 10^{-3}$ was obtained, where the errors represent experimental, heavy quark parameters, and theoretical uncertainties, respectively. The latter include estimates of the effects of subleading SFs, variations in the matching scales used in the calculation, and weak annihilation. The above determination of $\left|V_{u b}\right|$ is limited primarily by the precision on $m_{b}$. The study of the $E_{\gamma}$ moments in $B \rightarrow X_{s} \gamma$ decay at $B A B A R$ will soon become available and should improve our current knowledge of the $b$-quark mass. 


\section{Bibliography}

[1] Sakharov, A.D., ZhETF Pis. Red. 5, 32 (1967); and JETP Lett. 5, 24 (1967).

[2] J. Christenson, J. Cronin, V. Fitch, and R. Turlay, Phys. Rev. Lett. 13, 138 (1964).

[3] A.G. Cohen, D.B. Kaplan and A.E. Nelson, Ann. Rev. Nucl. Part. Sci. 43, 27 (1993).

[4] D. Boutigny et al. (BABAR Collab.), "BABAR Technical Design Report", SLAC-R-95457 (1995).

[5] M. Kobayashi and T. Maskawa, Prog. Theor. Phys. 49, 652 (1973).

[6] R. Fulton et al. (CLEO Collab.), Phys. Rev. Lett. 64, 16 (1990);

J. Bartelt et al. (CLEO Collab.), Phys. Rev. Lett. 71, 4111 (1993);

A. Bornheim et al. (CLEO Collab.), Phys. Rev. Lett. 88, 231803 (2002);

H. Albrecht et al. (ARGUS Collab.), Phys. Lett. B 234, 409 (1990);

and Phys. Lett. B 255, 297 (1991).

[7] B. Aubert et al. (BABAR Collab.), Phys. Rev. Lett. 92, 071802 (2004);

H. Kakuno et al. (Belle Collab.), Phys. Rev. Lett. 92, 101801 (2004).

[8] R. Kowalewski and S. Menke, Phys. Lett. B 541, 29 (2002).

[9] Glashow, S.L., Nucl. Phys. (International), Vol 22 No 4, 579 (1961).

[10] Weinberg, S., Phys. Rev. Lett. 19, 1264 (1967).

[11] Salam, A., N. Svartholm, ed. Stockholm: Almquist and Wiksell (1968).

[12] G. Arnison et al., Phys. Lett. B 122, 103 (1983);

M. Banner et al., Phys. Lett. B 122, 476 (1983).

[13] G. Arnison et al., Phys. Lett. B 126, 398 (1983);

P. Bagnaia et al., Phys. Lett. B 129, 130 (1983).

[14] The CDF Collaboration, Phys. Rev. Lett. 74, 2626 (1995).

[15] B. Aubert et al. (BABAR Collab.), Phys. Rev. Lett. 89, 201802 (2002);

K. Abe et al. (Belle Collab.), Phys. Rev. D 66, 071102 (2002). 
[16] Weinberg, S., Rev. Mod. Phys. 61, 1 (1989).

[17] Wolfenstein, L., Phys. Rev. Lett. 51, 1945 (1983).

[18] S. Eidelman et al. (Particle Data Group), Phys. Lett. B 592, 1 (2004).

[19] Heavy Flavour Averaging Group, http://www.slac.stanford.edu/xorg/hfag/ ; and arXiv:hep-ex/0412073 (2005).

[20] J. Charles et al. (CKM Fitter Group), Eur. Phys. Jour. C 41, 1 (2005).

[21] P.R. Burchat and J.D. Richman, Rev. Mod. Phys. 67, 893 (1995).

[22] N. Isgur, B. Grinstein, D. Scora, M. Wise, Phys. Rev. D 39, 799, 1989.

[23] D. Scora and N. Isgur, Phys. Rev. D 52, 2037 (1994).

[24] J. Shigemitsu et al., arXiv:hep-lat/04089019 (2004);

M. Okamoto et al., Nucl. Phys. Proc. Suppl. 140, 461 (2005).

[25] B. Aubert et al. (BABAR Collab.), Phys. Rev. D 69, 111103 (2004);

B. Aubert et al. (BABAR Collab.), Phys. Rev. D 69, 111104 (2004);

B. Aubert et al. (BABAR Collab.), Phys. Rev. Lett. 93, 011803 (2004).

[26] I. Bigi, M. Shifman, N. Uraltsev, Ann. Rev. Nucl. Part. Sci. 47, 591 (1997).

[27] J. Bjorken, Phys. Rev. D 17, 171 (1978).

[28] G. Altarelli et al., Nucl. Phys. B 208, 365 (1982).

[29] T. Mannel and M. Neubert, Phys. Rev. D 50, 2037 (1994).

[30] I. Bigi, M. Shifman, N. Uraltsev, and A. Vainshtein, Int. J. Mod. Phys. A9, 2467 (1994);

I. Bigi, M. Shifman and A. Vainstein, Phys. Lett. B 328, 431 (1994).

[31] M. Neubert, Int. J. Mod. Phys. A11, 4173 (1996).

[32] M.B. Voloshin, Phys. Lett. B 515, 74 (2001).

[33] C.W. Bauer, Z. Ligeti and M. Luke, Phys. Rev. D [64], 113004 (2001).

[34] A. Hoang, Z. Ligeti, and A. Manohar, Phys. Rev. Lett. 82, 277 (1999).

A. Hoang, Z. Ligeti, and A. Manohar, Phys. Rev. D 59, 074017 (1999).

[35] N. Uraltsev, Int. J. Mod. Phys. A11, 515 (1996);

N. Uraltsev, Int. J. Mod. Phys. A14, 464 (1999).

[36] Bigi, I., Workshop on the Derivation of $\left|V_{c b}\right|$ and $\left|V_{u b}\right|$, UND-HEP-BIG-99-05 (1999).

[37] M. Neubert and T. Becher, Phys. Lett. B 535, 127 (2002). 
[38] F. De Fazio and M. Neubert, JHEP 06, 017 (1999).

[39] A.L. Kagan and M. Neubert, Eur. Phys. Jour. C 7, 5 (1999).

[40] P. Koppenburg et al. (Belle Collab.), Phys. Rev. Lett. 93, 061803 (2004).

[41] A. Limosani and T. Nozaki, "Extraction of the $b$-quark shape function parameters using the Belle $B \rightarrow X_{s} \gamma$ photon energy spectrum", arXiv:hep-ex/0407052 (2005).

[42] C. W. Bauer, M. Luke and T. Mannel, Phys. Lett. B 543, 261 (2002);

A. Leibovich, A. Ligeti and M. Wise,Phys. Lett. B 539, 242 (2002)'

M. Neubert, Phys. Lett. B 543, 269 (2002).

[43] D. Boutigny et al. (BABAR Collab.), "The BABAR Physics Book", SLAC-R-504 (1998).

[44] B. Aubert et al. (BABAR Collab.), Nucl. Instr. Meth. A 479, 1 (2002).

[45] V. Re et al., Nucl. Instr. Meth. A 501, 14 (2003).

[46] D. Fortin, "dE/dx Calibration of the BaBar Drift Chamber: review and software", BABAR Analysis Document 617 (2003).

[47] S. Agostinelli et al. (GEANT4 Collab.), Nucl. Instr. Meth. A 506, 250 (2003).

[48] T. Sjöstrand, Comput. Phys. Commun. 82, 74 (1994).

[49] M. Neubert, private communication.

[50] L. Gibbons et al. (CLEO Collab.), AIP Conf. Proc. 722, 156 (2004).

[51] http://babar-hn.slac.stanford.edu:5090/HyperNews/get/semi_lept_decays/80/3.html Last visited 04/10/2005.

[52] Anastassov et al. (CLEO Collab.), Phys. Rev. Lett. 80, 4127 (1998).

[53] D. Abbaneo et al., "Combined results on $B$ hadron production rates and decay properties", SLAC-PUB-9500, CERN-EP-2001-050 (2001).

[54] J.L Goity and W. Roberts, Phys. Rev. D 51, 3459 (1995).

[55] D. Fortin, R. Kowalewksi, H. Lacker and F. Muheim, "Measurement of $\left|V_{u b}\right|$ from semileptonic $B$ decays using the lepton energy and $q^{2}$ ", BABAR Analysis Document 664 , version 6 (2004).

[56] G.C. Fox and S. Wolfram, Nucl. Phys. B 149, 413 (1979).

[57] B. Aubert et al. (BABAR Collab.), Phys. Rev. D 67, 031101 (2003).

[58] D. Fortin, R. Kowalewksi, H. Lacker and F. Muheim, "Charged track and neutral cluster selection in the $q^{2}-E_{\ell}$ analysis" BABAR Analysis Document 633 (2003). 
[59] A. Drescher et al. (ARGUS Collab.), Nucl. Instr. Meth. A 237, 464 (1985).

[60] R. Faccini et al., "Studies on $K_{S}^{0} \rightarrow \pi^{+} \pi^{-}$reconstruction", BABAR Analysis Document 19 (2000);

G. Cavoto et al., " $K_{S}^{0}$ efficiency studies in series 10", BABAR Analysis Document 706 (2003).

[61] U. Langenegger, "Cut based electron identification", BABAR Analysis Document 90 (2001);

T. Brandt, "Likelihood based electron identification", BABAR Analysis Document 396 (2002).

[62] S. Spanier and G. Mancinelli, "Kaon Identification in the BABAR Experiment", BABAR Analysis Document 116 (2001).

[63] F. Fabozzi et al., "Muon Identification in the BABAR Experiment", BABAR Analysis Document 60 (2000).

[64] B. Aubert et al. (BABAR Collab.), Phys. Rev. Lett. 94, 101801 (2005).

[65] J. Bartelt et al. (CLEO Collab.), Phys. Rev. Lett. 823746 (1999).

[66] B. Aubert et al. (BABAR Collab.), "Measurement of the Inclusive Electron Spectrum in Charmless Semileptonic B Decays Near the Kinematic Endpoint", presented at the $32^{\text {nd }}$ International Conference on High-Energy Physics (ICHEP 2004), arXiv:hepex/0408075 (2004).

[67] B. Aubert et al. (BABAR Collab.), "Measurement of $B \rightarrow D^{*+}$ form-factors in the semileptonic decay $\bar{B}^{0} \rightarrow D^{*+} \ell^{-} \bar{\nu}_{\ell}$ ", presented at the $32^{\text {nd }}$ International Conference on High-Energy Physics (ICHEP 2004), arXiv:hep-ex/0409047 (2004).

[68] M. Bona et al., "Report on the Tracking Efficiency Task Force for 2001", BABAR Analysis Document 324 (2001).

[69] http://www.slac.stanford.edu/BFR00T/www/Physics/Tools/BetaTools/ Last visited 04/10/2005.

[70] V. Brigljevic, "Study of $e^{+} e^{-} \rightarrow \phi \gamma$ events", BABAR Analysis Document 79 (2000).

[71] D. Fortin and M. Papagallo, "Study of $K_{L}^{0}$ energy deposition", BABAR Analysis Document 1055 (2004).

[72] E. Lohrmann, "Hochenergiephysik", Teubner, 2nd edition, Stuttgart (1988).

[73] E. Richter-Was, Phys. Lett. B 303, 163 (1993).

[74] E.S. Ginsberg, Phys. Rev. 142, 1035 (1966).

[75] B. Aubert et al. (BABAR Collab.), Phys. Rev. D 67, 032002 (2003). 
[76] C. Hearty, "Hadronic Event Selection and B-Counting for Inclusive Charmonium Measurements", BABAR Analysis Document 30 (2000).

[77] S.W. Bosch, B.O. Lange, M. Neubert and G. Paz, Nucl. Phys. B 699, 335 (2004);

B.O. Lange, M. Neubert and G. Paz, "Theory of Charmless Inclusive $B$ Decays and the Extraction of $\left|V_{u b}\right|$ ", hep-ph/0504071 (2005).

[78] M. Neubert, Phys. Lett. B 612, 13 (2005).

[79] M. Neubert, "Impact of four-quark shape functions on inclusive B decay spectra", arXiv:hep-ph/0411027 (2004);

S.W. Bosch, M. Neubert and G. Paz, JHEP 0411, 073 (2004).

[80] T.O. Meyer, "Limits on weak annihilation in inclusive charmless semileptonic $B$ decays", Cornell University thesis 05-1 (2005).

[81] B. Aubert et al. (BABAR Collab.), arXive:hep-ex/0408075 (2004);

B. Aubert et al. (BABAR Collab.), "Measurement of the Partial Branching Fraction for Inclusive Charmless Semileptonic $B$ Decays and Extraction of $\left|V_{u b}\right|$ ", Presented at the XXII International Symposium on Lepton-Photon Interactions at High Energy", arXive:hep-ex/0507017 (2005).

A. Limosani et al. (BELLE Collab.), Phys. Lett. B 621, 28 (2005);

I. Bizjak et al. (BELLE Collab.), "Measurement of the Inclusive Charmless Semileptonic Partial Branching Fraction of $B$ mesons and Determination of $\left|V_{u b}\right|$ using th Full Reconstruction Tag", Presented at the XXII International Symposium on Lepton-Photon Interactions at High Energy", arXive:hep-ex/0505088.

[82] A.K. Leibovich, I. Low and I.Z. Rothstein, Phys. Rev. D 61, 053006 (2000);

M. Neubert, Phys. Lett. B 543, 269 (2002).

[83] A.K. Leibovich, I. Low and I.Z. Rothstein, Phys. Rev. D 62, 014010 (2000);

A.K. Leibovich, I. Low and I.Z. Rothstein, Phys. Lett. B 486, 86 (2000);

A.K. Leibovich, I. Low and I.Z. Rothstein, Phys. Lett. B 513, 83 (2001).

[84] R. Dubitzky, E. Hill and U. Langenegger, "Measurement of $\left|V_{u b}\right|$ without recourse to structure functions", BABAR Analysis Document 880 (2005).

[85] P. Ball and R. Zwicky, " $\left|V_{u b}\right|$ and constraints on the leading-twist pion distribution amplitude", Submitted to Phys. Lett. B , arXive:hep-ph/0507076 (2005). 


\section{Appendix A}

\section{Defining $s_{\mathrm{h}}^{\max }$}

As shown in figure 2.4 , the $\mathrm{W}^{-*}$ carries a four-momentum $q$, such that in the $B$ rest frame

$$
q^{2}=m_{W^{*}}^{2}=\left(p_{\ell}+p_{\bar{\nu}_{\ell}}\right)^{2}=\left(p_{B}-p_{X}\right)^{2}=m_{B}^{2}+m_{X}^{2}-2 m_{B} E_{X},
$$

where $p_{B}$ is the four-momentum of the $\bar{B}$ meson and $E_{X}$ is the energy of $X_{u}$. Semileptonic decays are often characterized in terms of the invariant mass squared of the recoiling hadron, $s_{h}:$

$$
s_{h}=p_{X}^{2}=m_{X}^{2}=\left(p_{B}-\left(p_{\nu}+p_{l}\right)\right)^{2}
$$

where $p_{a}$ denotes the 4 -vector of particle $a$. Expanding equation A.2 in explicit terms, one obtains

$$
s_{h}=m_{B}^{2}+q^{2}-2 E_{B}\left(E_{l}+E_{\nu}\right)+2\left|\vec{p}_{B}\right| \sqrt{\left(E_{l}+E_{\nu}\right)^{2}-q^{2}} \cos \theta_{B-l \nu}
$$

where $\theta_{B-l \nu}$ is the angle between the $\bar{B}$ meson and the $l \nu$ system. If the lepton masses are ignored, the energy of the neutrino can be expressed as

$$
q^{2}=2 E_{l} E_{\nu}\left(1-\cos \theta_{l \nu}\right)
$$

such that $s_{h}$ can be written in the $\Upsilon(4 \mathrm{~S})$ rest frame as

$$
\begin{aligned}
s_{h}= & m_{B}^{2}+q^{2}-2 m_{B} \frac{1}{\sqrt{1-\beta^{2}}}\left(E_{l}+\frac{q^{2}}{2 E_{l}\left(1-\cos \theta_{l \nu}\right)}\right) \\
& +2 m_{B} \frac{\beta}{\sqrt{1-\beta^{2}}} \sqrt{E_{l}^{2}+E_{\nu}^{2}+2 E_{l} E_{\nu} \cos \theta_{l \nu}} \cos \theta_{B-l \nu} .
\end{aligned}
$$


In the above equation, $E_{B}$ and $\left|\vec{p}_{B}\right|$ have been written in terms of $m_{B}$ and $\beta$, the mass and speed of the $\mathrm{B}$ meson in the $\Upsilon(4 \mathrm{~S})$ rest frame $\left(\beta=\sqrt{1-\left(2 m_{B} / m_{\Upsilon(4 S)}\right)^{2}} \approx 0.06\right)$. Experimentally, three independent variables can be measured: $E_{l}, q^{2}$, and $m_{X}$ whereas the angles $\theta_{l \nu}$ and $\theta_{B-l \nu}$ cannot be measured directly. Nonetheless, one can compute the maximum kinematically allowed invariant mass squared of the hadronic recoil system, $s_{h}^{\max }$. This maximum occurs when $\cos \theta_{l \nu}=-1^{1}$, such that

$$
\begin{array}{r}
s_{h} \leq s_{h}^{\max }=m_{B}^{2}+q^{2}-2 m_{B} E_{l} \frac{1}{\sqrt{1-\beta^{2}}}\left(1-\beta \cos \theta_{B-l \nu}\right) \\
-2 m_{B} \frac{q^{2}}{4 E_{l}} \frac{1}{\sqrt{1-\beta^{2}}}\left(1+\beta \cos \theta_{B-l \nu}\right)
\end{array}
$$

Furthermore, the angle $\theta_{B-l \nu}$ can be eliminated. In the region where $E_{l}>\frac{\sqrt{q^{2}}}{2}\left(\frac{1+\beta}{1-\beta}\right)$, the maximum occurs when $\cos \theta_{B-l \nu}=+1$, which means

$$
s_{h}^{\max }=m_{B}^{2}+q^{2}-2 m_{B} E_{l} \sqrt{\frac{1-\beta}{1+\beta}}-2 m_{B}\left(\frac{q^{2}}{4 E_{l}}\right) \sqrt{\frac{1+\beta}{1-\beta}} .
$$

Similarly, in the region where $E_{l}<\frac{\sqrt{q^{2}}}{2}\left(\frac{1-\beta}{1+\beta}\right)$, the maximum occurs when $\cos \theta_{B-l \nu}=-1$ such that

$$
s_{h}^{\max }=m_{B}^{2}+q^{2}-2 m_{B} E_{l} \sqrt{\frac{1+\beta}{1-\beta}}-2 m_{B}\left(\frac{q^{2}}{4 E_{l}}\right) \sqrt{\frac{1-\beta}{1+\beta}} .
$$

Finally,

$$
s_{h} \leq s_{h}^{\max }=m_{B}^{2}+q^{2}-2 m_{B} \sqrt{q^{2}}
$$

is always satisfied.

\footnotetext{
${ }^{1}$ When the momenta of the lepton and the antineutrino are back-to-back: $\vec{p}_{l}=-\vec{p}_{\nu}$.
} 


\section{Appendix B}

\section{Particle Identification}

By default, all charged tracks recorded by the BABAR detector are assumed to be pions as they are the most common particles being produced: the $e: \pi: K: p$ production ratio is about $1: 5: 1: 0.1[61]$. Various algorithms are then applied to the charged tracks satisfying the selection described in section 5.3.2 to identify electons, kaons, muons and protons. If the track fail the identification requirements, the particle is then assumed to be a pion.

\section{B.1 Electron identification}

The electron selector [61] is arguably the most important algorithm in this analysis. Charged tracks are identified as electrons (or positrons) if they satisfy the following criteria:

1. After applying the $E / p$ calibration to the raw cluster energy, the ratio of the reconstructed energy over the momentum is the laboratory frame is $0.89<E / p<1.2$;

2. The number of crystals in the EMC clusters with energy deposited by the shower is $>3$

3. The shower lateral shape in the EMC has $0.0<\mathrm{LAT}<0.6$;

4. The ratio of the forth to the second Zernike moment, which describes the azimuthal shower profile, is $-10<A_{4,2}<0.11$;

5. The rate of energy loss as measured in the drift chamber is $540<\mathrm{dE} / \mathrm{dx}<860$; 
6. If the number of measured photons in the DIRC is $>10$, then the measured čerenkov angle is required to be within $3 \sigma$ of the electron hypothesis;

7. Electromagnetic showers start on average earlier in the EMC and are more concentrated around the impact point than showers of hadronic interactions. Therefore, they display a smaller azimuthal separation between the track impact point at the EMC and the center of gravity of the associated cluster. Hence, a requirement on $\Delta \phi=\phi_{\mathrm{EMC}}-\phi_{\text {clus }}$ is applied as a function of $p_{t}^{\mathrm{trk}}$.

\section{B.2 Kaon identification}

The kaon selector [62] is based on likelihood ratios between pions, kaons and protons. The selection was optimized to keep the mis-identification rate below $2 \%$ up to $p^{\text {lab }}>4 \mathrm{GeV}$. For candidate kaons with momenta $p^{\text {lab }}>0.6 \mathrm{GeV}$, identification is based primarily on angular information for the DIRC. The signature in the čerenkov detector is quite complex: the fit steps through the different particle hypotheses fitting the čerenkov angle and arrival time distribution of the photons associated to a track. The measurement of the čerenkov angle as a function of the kaon momentum is shown in figure B.1; the kaon band is labelled as region C. Region A is populated by tracks for which no fit is performed because the particles did not reach the DIRC or produced an amount of photon below threshold. Close to the threshold, kaons appear in the electron, muon and pion band with a rather wide spread (region B). The kaons decayed or interacted with the detector material prior to reaching the DIRC, but the daughter particles did not deviate much from their mother kaon direction. Region D correpond to the proton band. In this case, kaons underwent hadronic interactions which set free real protons. For momenta below $0.6 \mathrm{GeV}$, energy loss measurements from both the SVT and DCH are used, and at least 4 (11) SVT (DCH) hits must have been recorded. The efficiency for kaons with $p^{\text {lab }}<3 \mathrm{GeV}$ is better than $80 \%$ [62]. 


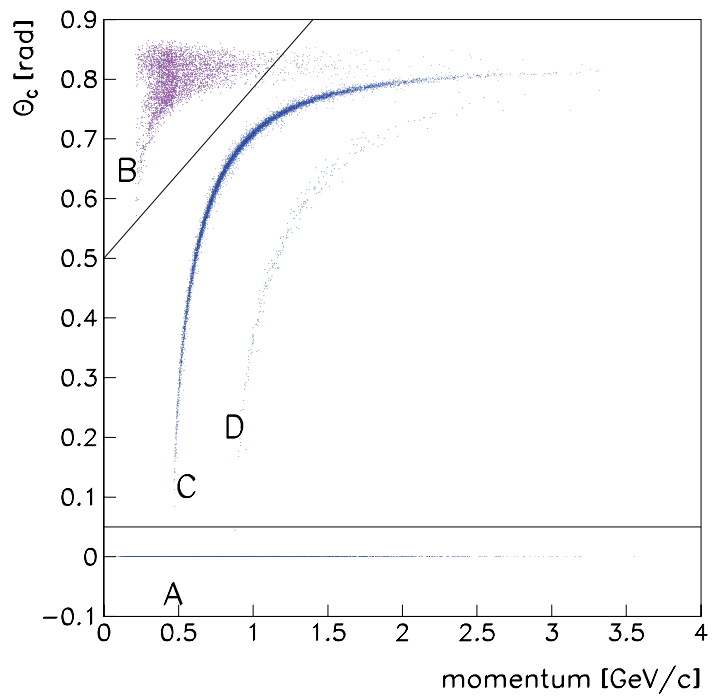

Figure B.1: Čerenkov angle measurement as a function of the momentum for simulated kaon tracks. The tracking information may be used in conjonction with čerenkov angle measurements to identify particles [44].

\section{B.3 Muon identification}

In the case of the muon algorithm [63], the following criteria must be satisfied:

1. For muon track candidates with matching EMC clusters, the energy released in the calorimeter is $0.04 \mathrm{GeV}<E_{\text {cal }}<0.4 \mathrm{GeV}$;

2. For muon track candidates with matching IFR clusters, the number of IFR layers with recorded hits is $N_{L} \geq 2$;

3. The measured number of interaction length traversed by the muon track candidate in the detector is $\lambda>2.2$;

4. The difference between the measured and expected interaction length traversed by the muon track candidate in the detector is $\Delta \lambda<1.0$;

5. The measured position by the IFR strips matches closely the track extrapolation, 
resulting in a $\chi^{2} /$ d.o.f. $<5$;

6. The track path measured by the IFR strips matches closely the track extrapolation, resulting in a $\chi_{\text {trk }}^{2} /$ d.o.f. $<5$;

7. The track path measured by the IFR strips matches closely a third order polynomial it of the IFR cluster, resulting in a $\chi_{\mathrm{fit}}^{2} /$ d.o.f. $<3$;

8. In order to decrease pion fake rate in the IFR forward endcap, the continuity of the track is of the track is required to be $T_{c}>0.3$ (see [63] for a description of this variable);

9. The average multiplicity of hit strips per layer is $\bar{m}<8$;

10. The measured standard deviation for $\bar{m}$ is $\sigma_{\bar{m}}<4$.

The above selection corresponds to the BABAR Tight Muon selection.

\section{B.4 Proton identification}

Proton identification is based upon $\mathrm{dE} / \mathrm{dx}$ and čerenkov angle measurements performed by the DCH and DIRC, respectively. For tracks in the momentum range $0.01 \mathrm{GeV}<p^{\text {lab }}<$ $0.9 \mathrm{GeV}, \theta_{C}$ is required to be zero. Protons are then identified as such if the measured $\mathrm{dE} / \mathrm{dx}$ is within $2 \sigma$ of the expected $\mathrm{dE} / \mathrm{dx}$ value for protons.

For charged tracks in the $0.9 \mathrm{GeV}<p^{\text {lab }}<3.0 \mathrm{GeV}$ momentum range, particles are identified as protons for the following cases:

- The measured $\theta_{C}=0$ and $\mathrm{dE} / \mathrm{dx}$ value is within $2 \sigma$ of the expected $\mathrm{dE} / \mathrm{dx}$ for protons, and the ratio of proton/kaon probabilities is $>20$;

- Both the measured $\theta_{C}$ and $\mathrm{dE} / \mathrm{dx}$ values are within $2 \sigma$ of the expected values;

- The measured $\mathrm{dE} / \mathrm{dx}$ value is within $2 \sigma$ of the expected $\mathrm{dE} / \mathrm{dx}$ for protons, and the ratios of proton/kaon probabilities as measured by both the DIRC and DCH are $>20$. 
Finally, for candidate tracks with momentum above $3 \mathrm{GeV}$, the $\mathrm{dE} / \mathrm{dx}$ and $\theta_{C}$ measurements are compared with expectated values using proton, kaon and pion mass hypotheses. If the proton mass hypothesis yields the best match in both cases, the the track is identified as a proton. 


\section{Appendix C}

\section{Alternative Results}

The signal branching ratio was extracted for three different electron energy requirements: 2.0, 2.1 and $2.2 \mathrm{GeV}$. In section 7, we discussed experimental uncertainties and the resulting systematics on the extracted branching ratio for an electron energy criterion of $E_{e}^{*}>2.1 \mathrm{GeV}$. Tables C.1 and C.2 outlines the yields and effiencies for a lower on on the electron energy of 2.0 and $2.1 \mathrm{GeV}$, respectively.

Systematic uncertainties using such requirements were evaluated according to the prescription outlined in section 7.2, and the results are summarized in tables C.3 and C.4. Hence, the signal $B \rightarrow X_{u} e \bar{\nu}_{e}$ branching ratio is

$$
\mathcal{B}_{u}^{\mathrm{SL}}=\left(2.69 \pm 0.22_{(\text {stat })} \pm 0.43_{(\text {syst })}\right) \times 10^{-3}
$$

\begin{tabular}{|l|l||l|l|}
\hline \multicolumn{2}{|c|}{ Inputs from data } & \multicolumn{2}{c|}{ Inputs from MC } \\
\hline \hline$N_{\text {cand }}^{\text {data }}$ & $8460 \pm 163$ & $M_{b k g}^{M C}$ & $5628 \pm 48$ \\
$N_{\text {sita }}^{\text {data }}$ & $17896 \pm 195$ & $M_{\text {side }}^{M C}$ & $18975 \pm 84$ \\
$\epsilon_{\text {dev }}^{\text {data }}$ & $(90.2 \pm 3.9) \times 10^{-3}$ & $\epsilon_{D e \nu}^{\mathrm{MC}}$ & $(90.6 \pm 1.9) \times 10^{-3}$ \\
& & $\epsilon_{u}^{\mathrm{MC}}$ & $(5.778 \pm 0.056) \times 10^{-3}$ \\
& & $f_{u}$ & $0.1602 \pm 0.0005$ \\
& & $\epsilon_{\mathrm{sig}}$ & $(31.8 \pm 0.3) \times 10^{-3}$ \\
& & $\epsilon_{\mathrm{sig}}$ & $(0.863 \pm 0.026) \times 10^{-3}$ \\
\hline
\end{tabular}

Table C.1: Data and Monte Carlo yields and efficiencies used for computing signal branching ratio for a lower bound on $E_{e}^{*}\left(\tilde{E}_{e}\right)$ of $2.0 \mathrm{GeV}(1.9 \mathrm{GeV})$. The uncertainties are purely statistical. 


\begin{tabular}{|c|c|c|c|}
\hline \multicolumn{2}{|c|}{ Inputs from data } & \multicolumn{2}{|r|}{ Inputs from MC } \\
\hline 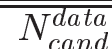 & $2616 \pm 138$ & $M_{b k g}^{M C}$ & $\overline{1156 \pm 21}$ \\
\hline $\begin{array}{l}\text { cana } \\
N_{\text {side }}^{\text {data }}\end{array}$ & $1145 \pm 84$ & $M_{\text {side }}^{\text {okg }}$ & $1226 \pm 21$ \\
\hline$\epsilon_{D e \nu}^{\text {data }}$ & $(90.2 \pm 3.9) \times 10^{-3}$ & $\epsilon_{D e \nu}^{\mathrm{MC}}$ & $(90.6 \pm 1.9) \times 10^{-3}$ \\
\hline & & $\epsilon_{u}^{\overline{\mathrm{MC}}}$ & $(3.359 \pm 0.047) \times 10^{-3}$ \\
\hline & & $f_{u}$ & $0.1145 \pm 0.0004$ \\
\hline & & $\epsilon_{\mathrm{sig}}$ & $(27.0 \pm 0.3) \times 10^{-3}$ \\
\hline & & $\epsilon \overline{\operatorname{sig}}$ & $(0.312 \pm 0.015) \times 10^{-3}$ \\
\hline
\end{tabular}

Table C.2: Data and Monte Carlo yields and efficiencies used for computing signal branching ratio for a lower bound on $E_{e}^{*}\left(\tilde{E}_{e}\right)$ of $2.2 \mathrm{GeV}(2.1 \mathrm{GeV})$. The uncertainties are purely statistical.

for $E_{e}^{*}>2.0 \mathrm{GeV}$, and

$$
\mathcal{B}_{u}^{\mathrm{SL}}=\left(2.60 \pm 0.30_{(\text {stat })} \pm 0.38_{(\text {syst })}\right) \times 10^{-3}
$$

for $E_{e}^{*}>2.2 \mathrm{GeV}$.

Using equation 2.35, the above results correspond to

$$
\left|V_{u b}\right|=\left(4.93 \pm 0.44_{(\text {expt })} \pm 0.24_{\left(\mathcal{B}_{u}^{S L} \rightarrow\left|V_{u b}\right|\right)}\right) \times 10^{-3}
$$

for $E_{e}^{*}>2.0 \mathrm{GeV}$, and

$$
\left|V_{u b}\right|=\left(4.84 \pm 0.45_{(\text {expt })} \pm 0.24_{\left(\mathcal{B}_{u}^{S L} \rightarrow\left|V_{u b}\right|\right)}\right) \times 10^{-3}
$$

for $E_{e}^{*}>2.2 \mathrm{GeV}$.

The results for the unfolded $\Delta \mathcal{B}_{u}^{\mathrm{SL}}$ corresponding to the above $E_{e}^{*}$ requirements translated to the $B$-meson rest-frame are

$$
\Delta \mathcal{B}\left(\tilde{E}_{e}>1.9 ; s_{\mathrm{h}}^{\max 0}<3.5\right)=\left(4.28 \pm 0.35_{(\text {stat })} \pm 0.58_{(\text {syst })}\right) \times 10^{-4},
$$

and

$$
\Delta \mathcal{B}\left(\tilde{E}_{e}>2.1 ; s_{\mathrm{h}}^{\max 0}<3.5\right)=\left(2.97 \pm 0.34_{(\text {stat })} \pm 0.29_{(\text {syst })}\right) \times 10^{-4} .
$$

The extraction of $\left|V_{u b}\right|$ based on the above partial branching ratio measurements is discussed in section 8.3. 


\begin{tabular}{|l|c|c|}
\hline Source of Systematics & $\sigma_{\mathcal{B}_{u}^{\text {SL }}}(\%)$ & $\sigma_{\Delta \mathcal{B}_{u}^{\text {SL }}}(\%)$ \\
\hline \hline 1a) Tracking efficiency & \pm 2.2 & \pm 2.2 \\
\hline 2a) Electron ID efficiency & \pm 1.1 & \pm 1.1 \\
\hline 3a) Charged particle ID & \pm 2.6 & \pm 2.6 \\
\hline 4a) Bremsstrahlung & \pm 2.2 & \pm 2.9 \\
\hline 5a) Neutrals reconstruction & \pm 6.6 & \pm 6.4 \\
\hline 6a) $K_{L}^{0}$ energy deposition & \pm 4.3 & \pm 4.3 \\
\hline 7a) B counting & \pm 1.1 & \pm 1.1 \\
\hline \hline A) Experimental systematics & \pm 9.0 & \pm 9.1 \\
\hline \hline 1b) $B \rightarrow X_{c} \ell \nu$ simulation & \pm 7.4 & \pm 7.4 \\
\hline 2b) Radiative corrections & \pm 4.3 & \pm 5.1 \\
\hline \hline B) Background simulation & \pm 8.6 & \pm 9.0 \\
\hline \hline 1c) $B \rightarrow X_{u} \ell \nu$ simulation & \pm 4.0 & \pm 4.4 \\
\hline 2c) Shape function uncertainties & \pm 9.0 & \pm 1.1 \\
\hline C) Signal simulation & \pm 9.8 & \pm 4.5 \\
\hline \hline Total $(A \oplus B \oplus C)$ & \pm 15.8 & \pm 13.6 \\
\hline
\end{tabular}

Table C.3: Summary of systematic uncertainties for a lower bound on the electron energy of $2.0 \mathrm{GeV}$. The systematics on the signal simulation also include the theoretical uncertainties associated with the uncertainty on $m_{b}$ and $a$. The total systematical uncertainty was computed as the quadratic sum of the uncertainties. In the case where a variation was taken only in one direction, the corresponding uncertainty was symmetrized. 


\begin{tabular}{|l|c|c|}
\hline Source of Systematics & $\sigma_{\mathcal{B}_{u}^{\text {SL }}}(\%)$ & $\sigma_{\Delta \mathcal{B}_{u}^{\mathrm{SL}}}(\%)$ \\
\hline \hline 1a) Tracking efficiency & \pm 1.1 & \pm 0.9 \\
\hline 2a) Electron ID efficiency & \pm 0.7 & \pm 0.9 \\
\hline 3a) Charged particle ID & \pm 2.2 & \pm 2.5 \\
\hline 4a) Bremsstrahlung & \pm 1.8 & \pm 2.1 \\
\hline 5a) Neutrals reconstruction & \pm 2.8 & \pm 2.8 \\
\hline 6a) $K_{L}^{0}$ energy deposition & \pm 1.1 & \pm 0.9 \\
\hline 7a) B counting & \pm 1.1 & \pm 1.1 \\
\hline \hline A) Experimental systematics & \pm 4.5 & \pm 4.7 \\
\hline \hline 1b) $B \rightarrow X_{c} \ell \nu$ simulation & \pm 3.6 & \pm 3.8 \\
\hline 2b) Radiative corrections & \pm 3.6 & \pm 3.9 \\
\hline \hline B) Background simulation & \pm 5.1 & \pm 5.4 \\
\hline \hline 1c) $B \rightarrow X_{u} \ell \nu$ simulation & \pm 4.9 & \pm 6.0 \\
\hline 2c) Shape function uncertainties & \pm 11.9 & \pm 2.7 \\
\hline C) Signal simulation & \pm 12.9 & \pm 6.6 \\
\hline \hline Total $(A \oplus B \oplus C)$ & \pm 14.6 & \pm 9.7 \\
\hline
\end{tabular}

Table C.4: Summary of systematic uncertainties for a lower bound on the electron energy of $2.2 \mathrm{GeV}$. The systematics on the signal simulation also include the theoretical uncertainties associated with the uncertainty on $m_{b}$ and $a$. The total systematical uncertainty was computed as the quadratic sum of the uncertainties. In the case where a variation was taken only in one direction, the corresponding uncertainty was symmetrized. 


\section{Partial Copyright License}

I hereby grant the right to lend my dissertation to users of the University of Victoria Library, and to make single copies only for such users or in response to a request from the Library of any other university, or similar institution, on its behalf or for one of its users. I further agree that permission for extensive copying of this thesis for scholarly purposes may be granted by me or a member of the University designated by me. It is understood that

copying or publication of this thesis for financial gain by the University of Victoria shall not be allowed without my written permission.

Title of Dissertation:

Determination of the CKM element $\left|V_{u b}\right|$

Author:

Dominique Fortin

October 5, 2005 